\title{
Switching Chemoselectivity Based on Ring Size: How to Make Ring-Fused Indoles Using Transition Metal-Mediated Cross-Coupling
}

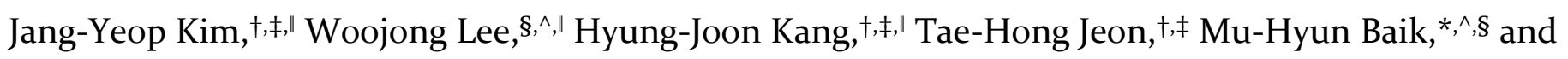
Cheon-Gyu Cho*,+‡

†Department of Chemistry, Hanyang University, 222 Wangsimni-ro, Seongdong-gu, Seoul 04763, Republic of Korea.

¥Center for New Directions in Organic Synthesis, Science Research Center (SRC), Seoul 04763, Republic of Korea.

§Department of Chemistry, Korea Advanced Institute of Science and Technology (KAIST), Daejeon 34141, Republic of Korea. ${ }^{\wedge}$ Center for Catalytic Hydrocarbon Functionalizations, Institute for Basic Science (IBS), Daejeon 34141, Republic of Korea.

"These authors contributed equally.

*E-mail: mbaik2805@kaist.ac.kr; ccho@hanyang.ac.kr

\section{Table of Contents}

\section{Experimental Procedures}

I. General Considerations

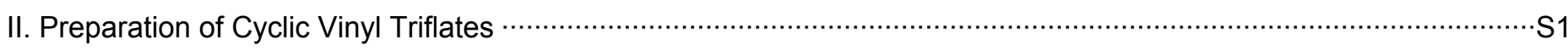

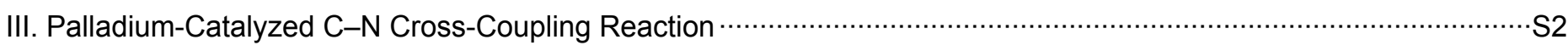

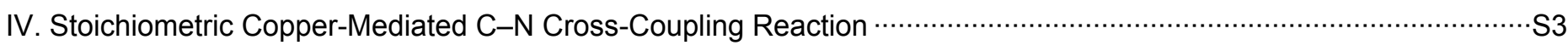

V. Procedures for the Cross-Coupling Reaction of Hydrazine with Cyclic Vinyl Triflates …………………………...S4

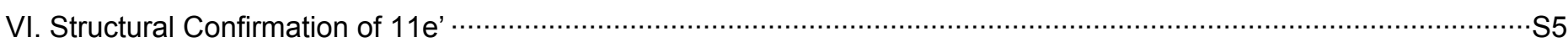

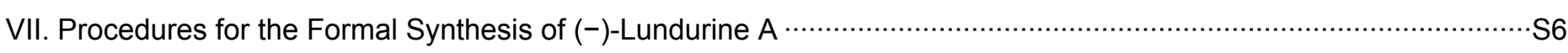

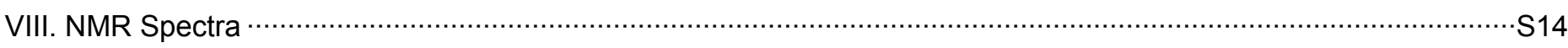

\section{Computational Information}

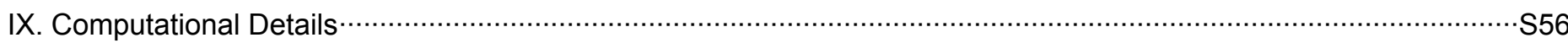

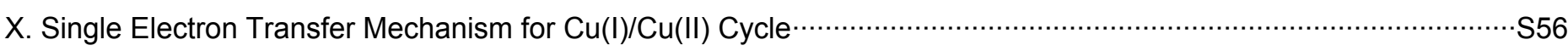

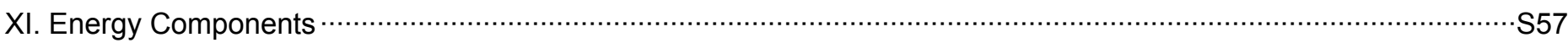

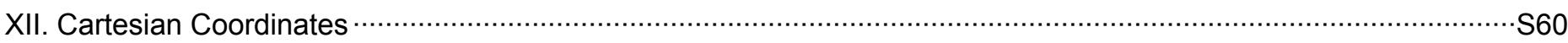

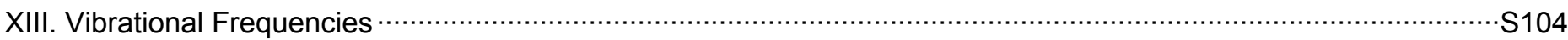

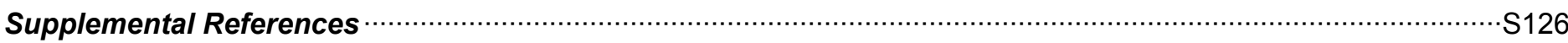




\section{Experimental Procedures}

\section{General Considerations}

Unless otherwise stated, reactions were performed under an argon atmosphere using freshly dried solvents. Tetrahydrofuran, dichloromethane, toluene, and ethyl ether were dried by passing through activated alumina columns. All other commercially obtained reagents were used as received. Reactions at elevated temperatures were heated in an oil bath. All reactions were monitored by thin-layer chromatography using EMD/Merck silica gel 60 F254 pre-coated plates $(0.25 \mathrm{~mm})$. The TLC spots were visualized under UV lamp or using staining solutions such as ceric ammonium molybdate solution, $p$-anisaldehyde solution, ninhydrin solution or potassium permanganate solution. Flash column chromatography was performed with indicated solvents using silica gel (GS60 - 40/75) purchased from Fuji Silysia Chemical. ${ }^{1} \mathrm{H}$ NMR and ${ }^{13} \mathrm{C}$ spectra were recorded on $400 \mathrm{MHz}$ (Bruker Avance III HD) spectrometer at $400 \mathrm{MHz}$ and $100 \mathrm{MHz}$, respectively. Chemical shifts are reported relative to tetramethylsilane $(0 \mathrm{ppm})$ or internal chloroform $\left({ }^{1} \mathrm{H}, \delta=7.26,{ }^{13} \mathrm{C}, \delta=77.16\right)$, internal DMSO $\left({ }^{1} \mathrm{H}, \delta=2.50,{ }^{13} \mathrm{C}, \delta=39.52\right)$ as indicated. Infrared spectra were recorded on Bruker LUMOS FT-IR spectrometer equipped with a microscope and mercury cadmium telluride (MCT) detector. High-resolution mass spectra were measured by using ESI-TOF method at Korean Basic Research Center, O-Chang, Republic of Korea.

\section{Preparation of Cyclic Vinyl Triflates}

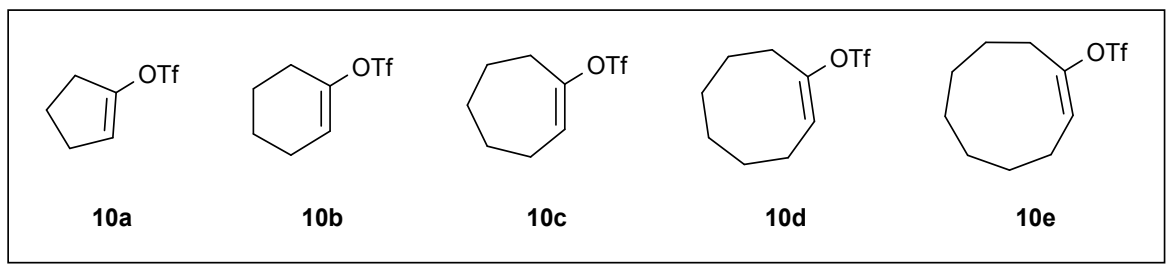

Scheme S1. Structures of cyclic vinyl triflates $10 \mathrm{a}-10 \mathrm{e}$.

Note: Cyclic vinyl triflates 10a, ${ }^{1} 10 b,{ }^{2} 10 c,{ }^{2}$ and $10 d^{2}$ were prepared by following the literature procedures.

(E)-Cyclonon-1-en-1-yl trifluoromethanesulfonate (10e)<smiles>O=C1CCCCCCCC1</smiles>

I

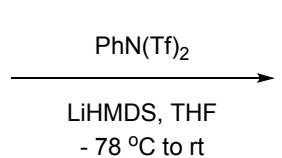

$-78^{\circ} \mathrm{C}$ to rt

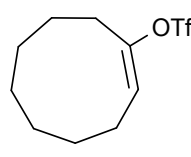

$10 \mathrm{e}$

Scheme S2. Synthesis of cyclic vinyl triflate $10 \mathrm{e}$.

To a solution of cyclononanone $(\mathbf{I}, 0.776 \mathrm{~g}, 5.53 \mathrm{mmol})$ in anhydrous THF $(5.5 \mathrm{~mL})$ was added LiHMDS (11.1 mL, $1.0 \mathrm{M}$ in THF, 1.5 equiv.) at $-78{ }^{\circ} \mathrm{C}$. After $30 \mathrm{~min}$ at $-78^{\circ} \mathrm{C}$, a solution of $\mathrm{PhNTf}_{2}\left(2.570 \mathrm{~g}, 7.19 \mathrm{mmol}, 1.3\right.$ equiv.) in anhydrous $\mathrm{THF}^{\circ}$ $(5.5 \mathrm{~mL})$ was cannulated to the mixture. The resulting mixture was warmed to rt, stirred for additional $1 \mathrm{~h}$, cooled to $0{ }^{\circ} \mathrm{C}$, quenched with saturated $\mathrm{NH}_{4} \mathrm{Cl}(\mathrm{aq})$ and extracted with EtOAc $(5 \mathrm{~mL} \times 3)$. The combined organic solution was washed with brine, dried over anhydrous $\mathrm{MgSO}_{4}$, filtered, concentrated in vacuo and chromatographed (100\% hexane) to give vinyl triflate $10 \mathrm{e}(0.963 \mathrm{~g}, 3.54 \mathrm{mmol})$ as a colorless oil in $64 \%$ yield.

$\mathrm{R}_{\mathrm{f}}=0.40\left(100 \%\right.$ hexane); ${ }^{1} \mathrm{H}$ NMR $\left(400 \mathrm{MHz}, \mathrm{CDCl}_{3}\right) \delta 5.63(\mathrm{t}, \mathrm{J}=9.2 \mathrm{~Hz}, 1 \mathrm{H}), 2.49(\mathrm{t}, \mathrm{J}=6.0 \mathrm{~Hz}, 2 \mathrm{H}), 2.21-2.16(\mathrm{~m}$, $2 \mathrm{H}), 1.72-1.66(\mathrm{~m}, 2 \mathrm{H}), 1.59-1.47(\mathrm{~m}, 8 \mathrm{H}) ;{ }^{13} \mathrm{C}$ NMR $\left(100 \mathrm{MHz}, \mathrm{CDCl}_{3}\right) \delta 151.2,123.3,121.7,120.1,116.9,113.8,29.4$ 25.5, 25.2, 24.9, 24.87, 24.83, 23.6; FT-IR $\left(\mathrm{CH}_{2} \mathrm{Cl}_{2}\right)$ 2938, 1685, 1418, 1214, 1144, 985, 903, 612 cm-1; HRMS (ESI-TOF) - no corresponding molecular ion peak observed. 


\section{Palladium-Catalyzed C-N Cross-Coupling Reaction}

A $4 \mathrm{~mL}$ vial was charged with a stir bar, vinyl triflate $10 \mathrm{~b} / 10 \mathrm{~d}(0.30 \mathrm{mmol})$, phenyl hydrazine $7(2.0$ equiv. $), \mathrm{Pd}_{2}\left(\mathrm{dba}_{3}(2.5\right.$ $\mathrm{mol} \%$ ), ligand (6.0 mol\%), and $\mathrm{Cs}_{2} \mathrm{CO}_{3}$ (2.0 equiv.). For $\mathbf{L} 12-\mathbf{L} 14$, ligated catalysts $\mathrm{Pd}(\mathbf{L})_{2}(5.0 \mathrm{~mol} \%)$ were subjected to the reaction. The reaction vessel was sealed with a screw cap with PTFE septum, evacuated and backfilled with argon (total of three times). Then, degassed anhydrous toluene $(1.0 \mathrm{~mL}$ ) was added to the vial which was immediately sealed with a new screw cap. The solution was stirred at $110^{\circ} \mathrm{C}$ for $12 \mathrm{~h}$, cooled to rt, diluted with EtOAc, filtered through a plug of silica gel, and purified by flash column chromatography. The reaction conditions were developed based on our previous works. ${ }^{3}$

Table S1. Screening of ligands for palladium-catalyzed C-N cross-coupling reaction. ${ }^{a}$
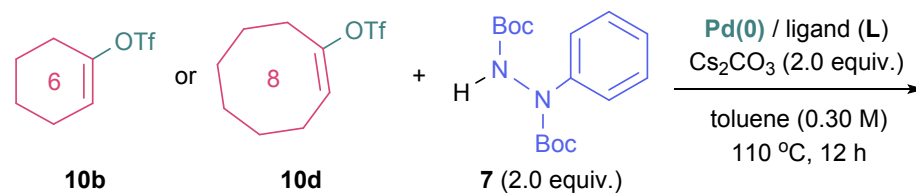

$110^{\circ} \mathrm{C}, 12 \mathrm{~h}$<smiles>CC1(C)c2ccccc2Oc2c(-c3ccccc3)cccc21</smiles>

XantPhos (L1)

11b, $17 \%^{b}$

11d, trace ${ }^{b}$<smiles>CCCCc1ccccc1-c1ccccc1N(C)C</smiles>

${ }^{t}$ BuDavePhos (L6)

11b, trace

11d, trace
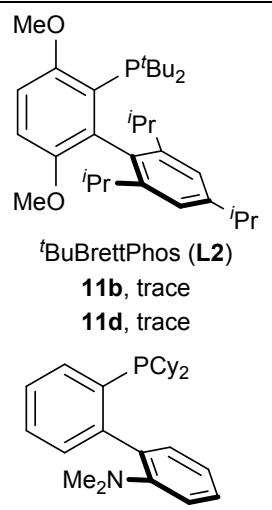

DavePhos (L7)

11b, trace

11d, trace
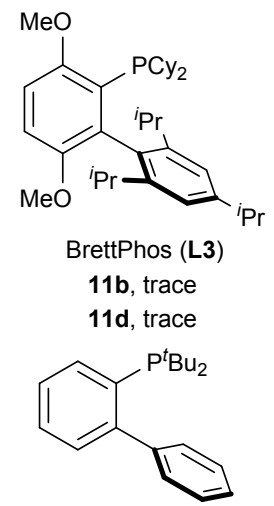

JohnPhos (L8)

11b, trace

11d, trace

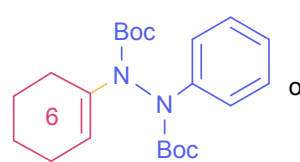

11b

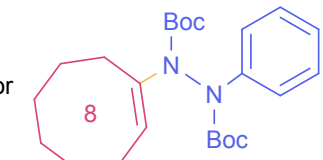

11d<smiles>COc1ccc(P(c2ccc(OC)cc2)c2ccc(OC)cc2)cc1</smiles><smiles>Cc1ccccc1P(c1ccccc1C)c1ccccc1C</smiles>

$\mathrm{P}$ (o-tolyl) ${ }_{3}$ (L12)

$11 \mathrm{~b}$, trace

11d, trace

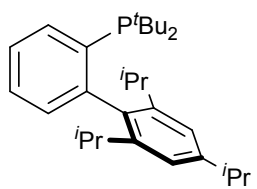

${ }^{t}$ BuXPhos (L4)

$11 \mathrm{~b}$, trace

11d, trace

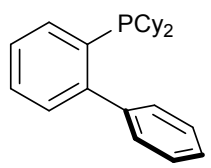

CyJohnPhos (L9)

11b, trace

11d, trace

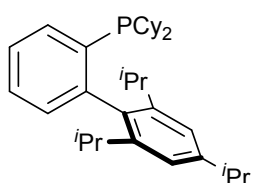

XPhos (L5)

11b, trace

11d, trace

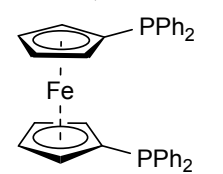

dppf (L10)

11b, trace

11d, trace

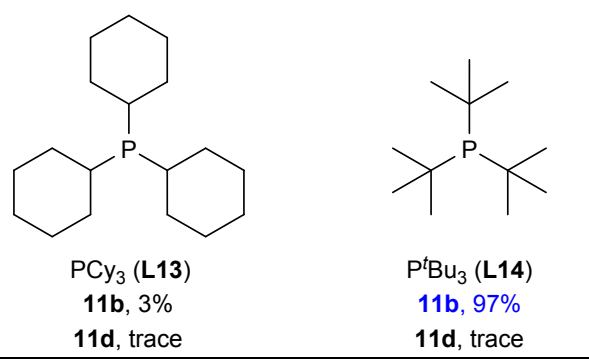

aYields after purification by chromatography. ${ }^{b}$ The reaction was subjected to previously reported conditions. ${ }^{3}$

Note: Ligands (L1-L11) and ligated palladium catalyst $P d(\mathbf{L 1 2}-\mathbf{L 1 4})_{2}$ were obtained from commercial sources. 


\section{Stoichiometric Copper-Mediated C-N Cross-Coupling Reaction}

A $4 \mathrm{~mL}$ vial was charged with a stir bar, vinyl triflate $10 \mathrm{~b} / 10 \mathrm{~d}(0.30 \mathrm{mmol})$, phenyl hydrazine 7 (2.0 equiv.), Cul (1.0 equiv.), ligand (2.0 equiv., if solid), and $\mathrm{K}_{3} \mathrm{PO}_{4}$ (2.0 equiv.). The reaction vessel was sealed with a screw cap with PTFE septum, evacuated and backfilled with argon (total of three times). Then, degassed anhydrous toluene (1.0 $\mathrm{mL})$ and ligand (2.0 equiv., if liquid) were added to the vial which was immediately sealed with a new screw cap. The solution was stirred at $50{ }^{\circ} \mathrm{C}$ for $16 \mathrm{~h}$, cooled to rt, diluted with EtOAc, filtered through a plug of silica gel, and purified by flash column chromatography.

Table S2. Screening of ligands for stoichiometric copper-mediated C-N cross-coupling reaction. ${ }^{a}$

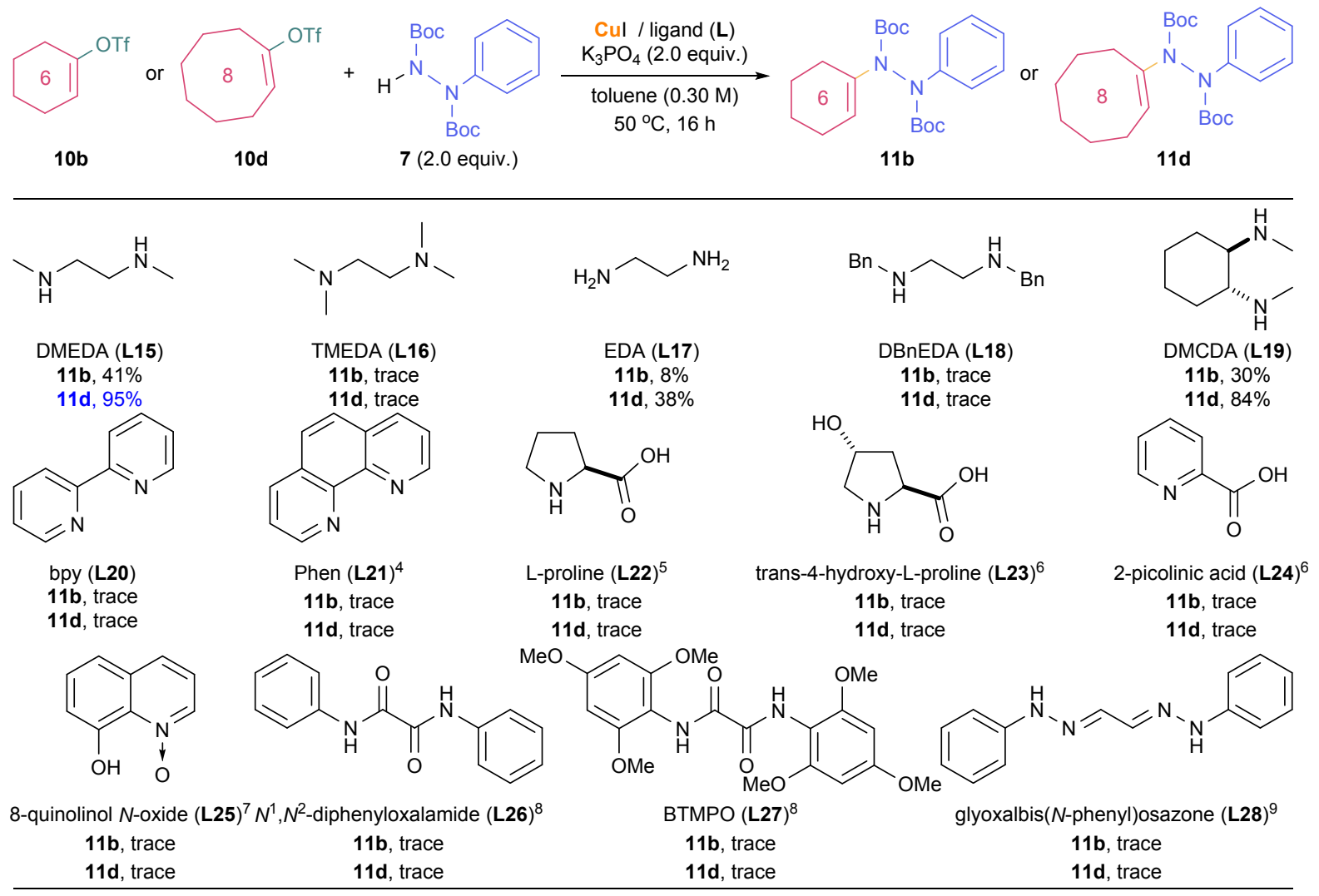

aYields after purification by chromatography. L15: DMEDA $=N^{1}, N^{2}$-dimethylethylenediamine. L16: TMEDA $=N^{1}, N^{1}, N^{2}, N^{2}$ tetramethylethylenediamine. L17: EDA = ethylenediamine. L18: DBnEDA = $N^{1}, N^{2}$-dibenzylethylenediamine. L19: DMCDA $=(1 R, 2 R)-N^{1}, N^{2}$-dimethyl-1,2-cyclohexanediamine. L20: bpy = 2,2'-bipyridine. L21: Phen = 1,10-phenanthroline. L27: BTMPO $=N^{1}, N^{2}$-bis(2,4,6-trimethoxyphenyl)oxalamide.

Note: Ligands $L 15, L 16, L 17, L 19, L 20, L 21, L 22, L 23$, and $L 24$ were obtained from commercial sources. The syntheses of ligands $\mathbf{L} 18,{ }^{10} \mathbf{L} 25,{ }^{11} \mathbf{L} 26,{ }^{12} \mathbf{L} 27,{ }^{12}$ and $\mathbf{L} \mathbf{2 8}^{13}$ have been previously reported. 


\section{Procedures for the Cross-Coupling Reaction of Hydrazine with Cyclic Vinyl Triflates}

Di-tert-butyl (E)-1-(cyclooct-1-en-1-yl)-2-phenylhydrazine-1,2-dicarboxylate (11d)

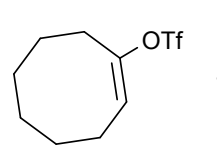

10d<smiles>CC(C)(C)OC(=O)NN(C(=O)OC(C)(C)C)c1ccccc1</smiles>

7 (2.0 equiv.)

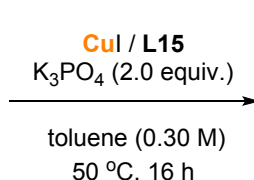

$50^{\circ} \mathrm{C}, 16 \mathrm{~h}$

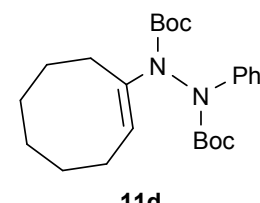

11d

Scheme S3. Coupling reaction between vinyl triflate $10 \mathrm{~d}$ and aryl hydrazine 7 under stoichiometric condition.

A $4 \mathrm{~mL}$ vial was charged with stir bar, vinyl triflate $10 \mathrm{~d}(77.5 \mathrm{mg}, 0.30 \mathrm{mmol})$, phenyl hydrazine $7(184.4 \mathrm{mg}, 0.60 \mathrm{mmol}$, 2.0 equiv.), Cul ( $57.1 \mathrm{mg}, 0.30 \mathrm{mmol}, 1.0$ equiv.) and $\mathrm{K}_{3} \mathrm{PO}_{4}(127.4 \mathrm{mg}, 0.60 \mathrm{mmol}, 2.0$ equiv.) at rt. The reaction vessel was sealed with a screw cap with PTFE septum, evacuated and backfilled with argon (total of three times). Then, degassed anhydrous toluene $(1.0 \mathrm{~mL})$ and $\mathrm{L} 15(0.06 \mathrm{~mL}, 0.60 \mathrm{mmol}, 2.0$ equiv.) were added to the vial which was immediately sealed with a new screw cap. The solution was stirred at $50^{\circ} \mathrm{C}$ for $16 \mathrm{~h}$, cooled to rt, diluted with EtOAc, filtered through a plug of silica gel, concentrated in vacuo, and chromatographed (hexane $/ \mathrm{Et}_{2} \mathrm{O}=8: 1$ ) to give ene-hydrazine 11d (118.7 mg, 0.29 $\mathrm{mmol}$ ) as colorless oil in $95 \%$ yield. $\mathrm{R}_{\mathrm{f}}=0.5$ (hexane $/ \mathrm{Et}_{2} \mathrm{O}=5: 1$ ).

${ }^{1} \mathrm{H}$ NMR $\left(400 \mathrm{MHz}, \mathrm{DMSO}-\mathrm{d}_{6}, 70{ }^{\circ} \mathrm{C}\right) \delta 7.35-7.27(\mathrm{~m}, 4 \mathrm{H}), 7.18-7.14(\mathrm{~m}, 1 \mathrm{H}), 5.44(\mathrm{t}, \mathrm{J}=8.4 \mathrm{~Hz}, 1 \mathrm{H}), 2.28-2.25(\mathrm{~m}$, $2 \mathrm{H}), 2.08-2.02(\mathrm{~m}, 2 \mathrm{H}), 1.48-1.46(\mathrm{~m}, 26 \mathrm{H}) ;{ }^{13} \mathrm{C}$ NMR $\left(100 \mathrm{MHz}\right.$, DMSO-d $\left.\mathrm{d}^{6}, 70{ }^{\circ} \mathrm{C}\right) \delta 152.6,152.3,140.4,138.0,127.8$, 124.9, 124.1, 123.1, 80.9, 80.5, 28.7, 27.9, 27.6, 27.4, 26.1, 25.3, 25.1, 24.8; FT-IR $\left(\mathrm{CHCl}_{3}\right)$ 2930, 1726, 1344, 1165, 1055, $757 \mathrm{~cm}^{-1}$; HRMS (ESI-TOF) m/z: [M + Na] $]^{+}$Calcd for $\mathrm{C}_{24} \mathrm{H}_{36} \mathrm{~N}_{2} \mathrm{O}_{4} \mathrm{Na} 439.2573$; Found 439.2574.

\section{Di-tert-butyl (E)-1-(cyclohex-1-en-1-yl)-2-phenylhydrazine-1,2-dicarboxylate (11b)}<smiles>CC(C)(C)OC(=O)N(C1=CCCCC1)N(C(=O)OC(C)(C)C)c1ccccc1</smiles>

$11 \mathrm{~b}$ $41 \%$ yield (hexane $/$ EtOAc $=15: 1), 16 \mathrm{~h}$, colorless oil. $\mathrm{R}_{\mathrm{f}}=0.4$ (hexane $/$ EtOAc $\left.=10: 1\right)$.

${ }^{1} \mathrm{H}$ NMR $\left(400 \mathrm{MHz}, \mathrm{CDCl}_{3}\right) \delta 7.36(\mathrm{~m}, 2 \mathrm{H}), 7.33-7.29(\mathrm{~m}, 2 \mathrm{H}), 5.63$ (brs, 1H), $2.26-2.05(\mathrm{~m}, 4 \mathrm{H}), 1.67$ $-1.61(\mathrm{~m}, 4 \mathrm{H}), 1.52(\mathrm{~s}, 9 \mathrm{H}), 1.49 / 1.48$ (rotamers, $2 x \mathrm{~s}, 9 \mathrm{H}) ;{ }^{13} \mathrm{C}$ NMR $\left(100 \mathrm{MHz}, \mathrm{CDCl}_{3}\right) \delta 153.3,141.5$, $137.4,128.5,125.5,123.8 / 123.3,121.5,81.9,81.5,28.4,28.3,26.4,24.6,22.9,21.8$; spectral data are consistent with those reported in the literature. ${ }^{3}$

\section{Di-tert-butyl 1-(cyclohept-1-en-1-yl)-2-phenylhydrazine-1,2-dicarboxylate (11c)}

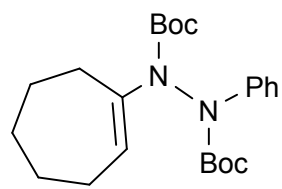

$11 \mathrm{c}$
94\% yield (hexane $/$ EtOAc $=15: 1), 16 \mathrm{~h}$, colorless oil. $\mathrm{R}_{\mathrm{f}}=0.4$ (hexane $/$ EtOAc $=10: 1$ ).

${ }^{1} \mathrm{H}$ NMR $\left(400 \mathrm{MHz} \mathrm{CDCl}_{3}\right) \delta 7.34-7.29(\mathrm{~m}, 4 \mathrm{H}), 7.17-7.16(\mathrm{~m}, 1 \mathrm{H}), 5.75(\mathrm{~s}, 1 \mathrm{H}), 2.35(\mathrm{~m}, 2 \mathrm{H})$ $2.06-2.04(\mathrm{~m}, 2 \mathrm{H}), 1.69-1.68(\mathrm{~m}, 2 \mathrm{H}), 1.51(\mathrm{~s}, 18 \mathrm{H}), 1.41-1.25(\mathrm{~m}, 4 \mathrm{H}) ;{ }^{13} \mathrm{C} \mathrm{NMR}(100 \mathrm{MHz}$, $\left.\mathrm{CDCl}_{3}\right) \delta 153.5,144.1,128.6,126.2,125.7,125.0,123.8,82.0,81.6,32.1,30.3,28.5,28.4,26.9$, $26.7,26.2$; spectral data are consistent with those reported in the literature. ${ }^{3}$

Di-tert-butyl (E)-1-(cyclonon-1-en-1-yl)-2-phenylhydrazine-1,2-dicarboxylate (11e) and tert-Butyl (Z)-2-(cyclonon-1en-1-yl)-1-phenylhydrazine-1-carboxylate (pre-11e')

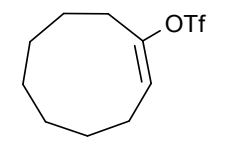

$10 \mathrm{e}$

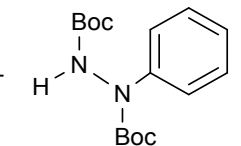

7

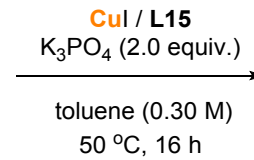
$50^{\circ} \mathrm{C}, 16 \mathrm{~h}$

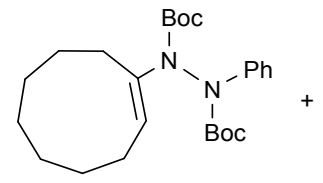

11e $(40 \%)$

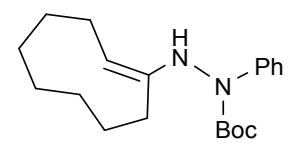

pre-11e' (42\%)

Scheme S4. Coupling reaction between vinyl triflate $10 \mathrm{e}$ and aryl hydrazine 7 under stoichiometric condition.

After $16 \mathrm{~h}, 11 \mathrm{e}$ (191 mg, 40\% yield) and pre-11e' (150 mg, 42\% yield) were obtained after column chromatography (hexane $\left./ \mathrm{Et}_{2} \mathrm{O}=8: 1\right)$. 
11e: a colorless oil, $\mathrm{R}_{\mathrm{f}}=0.5$ (hexane $/ \mathrm{Et}_{2} \mathrm{O}=5: 1$ ).

${ }^{1} \mathrm{H}$ NMR $\left(400 \mathrm{MHz}\right.$, DMSO-d $\left.6,70{ }^{\circ} \mathrm{C}\right) \delta 7.35-7.26(\mathrm{~m}, 4 \mathrm{H}), 7.18-7.14(\mathrm{~m}, 1 \mathrm{H}), 5.34(\mathrm{t}, \mathrm{J}=8.8 \mathrm{~Hz}, 1 \mathrm{H}), 2.26-2.16(\mathrm{~m}$, 2H), $2.15-1.99(\mathrm{~m}, 2 \mathrm{H}), 1.48-1.41(\mathrm{~m}, 28 \mathrm{H}) ;{ }^{13} \mathrm{C}$ NMR $\left(100 \mathrm{MHz}\right.$, DMSO-d $\left.6.70{ }^{\circ} \mathrm{C}\right) \delta 152.8,152.3,140.3,137.5,127.8$, 125.1, 123.4, 80.9, 80.5, 27.6, 27.4, 25.9, 25.2, 25.1, 24.5, 24.3, 24.1, 23.4, 23.2; FT-IR $\left(\mathrm{CH}_{2} \mathrm{Cl}_{2}\right)$ 2934, 1726, 1491, 1346, 1165, $759 \mathrm{~cm}^{-1}$; HRMS (ESI-TOF) m/z: [M + Na] $]^{+}$Calcd for $\mathrm{C}_{25} \mathrm{H}_{38} \mathrm{~N}_{2} \mathrm{O}_{4} \mathrm{Na} 453.2729$; Found 453.2728.

pre-11e': a colorless oil. $\mathrm{R}_{\mathrm{f}}=0.15$ (hexane / $\mathrm{Et}_{2} \mathrm{O}=5: 1$ ).

${ }^{1} \mathrm{H}$ NMR $\left(400 \mathrm{MHz}\right.$, DMSO-d $\left.\mathrm{d}_{6}, 70^{\circ} \mathrm{C}\right) \delta 7.42-7.32(\mathrm{~m}, 4 \mathrm{H}), 7.16(\mathrm{t}, \mathrm{J}=7.2 \mathrm{~Hz}, 1 \mathrm{H}), 5.11$ (brs, $\left.1 \mathrm{H}\right), 2.25-1.24(\mathrm{~m}, 23 \mathrm{H})$; ${ }^{13} \mathrm{C}$ NMR $\left(100 \mathrm{MHz}\right.$, DMSO-d $\left.6,70{ }^{\circ} \mathrm{C}\right) \delta 152.5,146.9,142.0,127.9,124.7,122.6,117.2,80.9,33.9,30.3,27.5,27.3,25.3$, 24.0, 23.8; FT-IR $\left(\mathrm{CH}_{2} \mathrm{Cl}_{2}\right) 3320,2932,1726,1499,1369,1161,1118,759 \mathrm{~cm}^{-1}$; HRMS (ESI-TOF) - no corresponding molecular ion peak was observed.

\section{Structural Confirmation of 11e'}

\section{Boc-Protection of pre-11e'}

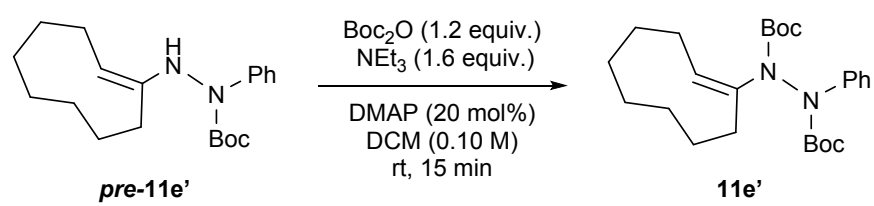

Scheme S5. Boc-protection of singly masked isomer obtained from reaction of vinyl triflate $10 \mathrm{e}$ and aryl hydrazine 7 under stoichiometric copper-mediated coupling reaction.

\section{Di-tert-butyl (Z)-1-(cyclonon-1-en-1-yl)-2-phenylhydrazine-1,2-dicarboxylate (11e')}

To a solution of pre-11e' $(61.7 \mathrm{mg}, 0.19 \mathrm{mmol})$ in DCM $(1.9 \mathrm{~mL}, 0.1 \mathrm{M})$ were added TEA (0.04 mL, $0.30 \mathrm{mmol}, 1.6$ equiv.), DMAP (4.6 mg, $0.038 \mathrm{mmol}, 20 \mathrm{~mol} \%)$ and di-tert-butyl dicarbonate $(0.05 \mathrm{~mL}, 0.23 \mathrm{mmol}, 1.2$ equiv.) at rt. After $15 \mathrm{~min}$, the reaction mixture was quenched by water and extracted with $\mathrm{DCM}(3 \mathrm{~mL} \times 2)$. The combined organic solution was washed with brine, dried over anhydrous $\mathrm{MgSO}_{4}$, filtered, concentrated in vacuo and chromatographed (hexane $/ \mathrm{Et}_{2} \mathrm{O}=5: 1$ ) to give $11 e^{\prime}$ as a light yellow oil in $98 \%$ yield $(79.1 \mathrm{mg}, 0.18 \mathrm{mmol}) . \mathrm{R}_{\mathrm{f}}=0.62$ (hexane $\left./ \mathrm{Et}_{2} \mathrm{O}=5: 1\right) ;{ }^{1} \mathrm{H} \mathrm{NMR}\left(400 \mathrm{MHz}, \mathrm{CDCl}_{3}\right) \delta$ $7.46-7.26(\mathrm{~m}, 4 \mathrm{H}), 7.15-7.14(\mathrm{~m}, 1 \mathrm{H}), 5.16-5.07(\mathrm{~m}, 1 \mathrm{H}), 2.25-1.11(\mathrm{~m}, 51 \mathrm{H}) ;{ }^{13} \mathrm{C} \mathrm{NMR}\left(100 \mathrm{MHz}, \mathrm{CDCl}_{3}\right) \delta 150.9$, $150.1,147.6,146.8,128.6,125.4,121.8,121.5,119.5,85.2,84.6,82.9,34.7,31.4,31.1,28.3,28.2,28.1,28.0,28.0,27.5$, 25.8, 25.4, 24.9, 24.3, 24.1, 23.8; FT-IR $\left(\mathrm{CH}_{2} \mathrm{Cl}_{2}\right)$ 2934, 1730, 1495, 1342, 1254, 1122, $759 \mathrm{~cm}^{-1}$; HRMS (ESI-TOF) - no corresponding molecular ion peak was observed.

\section{Indolization of $11 \mathrm{e}$ and $11 \mathrm{e}^{\prime}$}

\section{$5,6,7,8,9,10,11,12-$ Octahydrocyclonona[b]indole $(12 e)$}

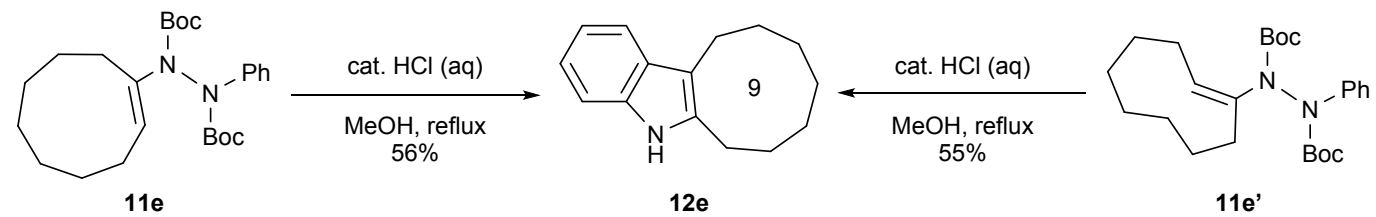

Scheme S6. Structural identification of $12 \mathrm{e}$ by Fischer indolization of $11 \mathrm{e}$ and $11 \mathrm{e}$.

A mixture of the 11e' $(94.1 \mathrm{mg}, 0.22 \mathrm{mmol})$ in $\mathrm{MeOH}(2.2 \mathrm{~mL}, 0.1 \mathrm{M})$ was added conc. $\mathrm{HCl}$ (2 drops) and the reaction was stirred at $65{ }^{\circ} \mathrm{C}$ for $3 \mathrm{~h}$. The solution was cooled to it and concentrated in vacuo. The mixture was diluted with DCM, neutralized with $\mathrm{NaHCO}_{3}(\mathrm{aq})$, and extracted with $\mathrm{DCM}(5 \mathrm{~mL} \times 2)$. The combined organic solution was washed with brine, dried over anhydrous $\mathrm{MgSO}_{4}$, filtered, concentrated in vacuo and chromatographed (hexane / $\left.\mathrm{Et}_{2} \mathrm{O}=5: 1\right)$ to give 12e $(26.1$ $\mathrm{mg}, 0.12 \mathrm{mmol}$ ) as a yellow oil in $56 \%$ yield. The indole product $12 \mathrm{e}$ was found to be identical to the indole product obtained from isomeric 11e with respect to the ${ }^{1} \mathrm{H} N M R,{ }^{13} \mathrm{C} N M R$, IR, and HRMS data as shown below.

12e. $\mathrm{R}_{\mathrm{f}}=0.48\left(\right.$ hexane $\left./ \mathrm{Et}_{2} \mathrm{O}=5: 1\right) ;{ }^{1} \mathrm{H}$ NMR $\left(400 \mathrm{MHz}, \mathrm{CDCl}_{3}\right) \delta 7.62($ brs, $1 \mathrm{H}), 7.49-7.47(\mathrm{~m}, 1 \mathrm{H}), 7.28-7.25(\mathrm{~m}, 1 \mathrm{H})$, 
$7.12-7.06(\mathrm{~m}, 2 \mathrm{H}), 2.88-2.82(\mathrm{~m}, 4 \mathrm{H}), 1.76-1.69(\mathrm{~m}, 4 \mathrm{H}), 1.50-1.35(\mathrm{~m}, 6 \mathrm{H}) ;{ }^{13} \mathrm{C} \mathrm{NMR}\left(100 \mathrm{MHz}, \mathrm{CDCl}_{3}\right) \delta 135.9$, 135.5, 128.9, 120.9, 119.0, 118.1, 112.4, 110.3, 27.4, 27.1, 26.1, 25.7, 25.4, 25.0, 22.7; FT-IR $\left(\mathrm{CHCl}_{3}\right) 3409,2930,1701$, 1463, 1338, 1010, $742 \mathrm{~cm}^{-1}$; HRMS (ESI-TOF) m/z: [M+] Calcd for $\mathrm{C}_{15} \mathrm{H}_{19} \mathrm{~N}$ 213.1517; Found 213.1519.

\section{Procedures for the Formal Synthesis of (-)-Lundurine A}

\section{Ethyl (R)-2-cinnamyl-5-oxopyrrolidine-2-carboxylate (14)}

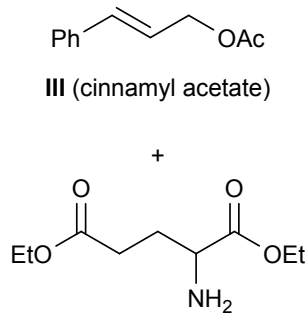

13 (diethyl glutamate)

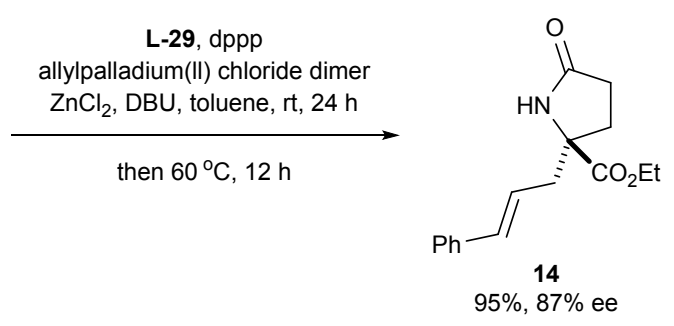<smiles>O=Cc1cc2ccccc2c(-c2c(O)c([N+](=O)[O-])cc3ccccc23)c1O</smiles>

Scheme S7. Formation of chiral lactam 14.

A mixture of allylpalladium(II) chloride dimer $(0.10 \mathrm{~g}, 0.28 \mathrm{mmol})$ and dppp $(0.23 \mathrm{~g}, 0.57 \mathrm{mmol})$ in toluene $(20 \mathrm{~mL})$ was stirred for $30 \mathrm{~min}$ at $0{ }^{\circ} \mathrm{C}$. To a separate flask charged with cinnamyl acetate (III, $1.00 \mathrm{~g}, 5.68 \mathrm{mmol}$ ), amino acid ester 13 $(1.73 \mathrm{~g}, 8.51 \mathrm{mmol})$ and toluene $(30 \mathrm{~mL})$ were added $\mathrm{L}-29(0.22 \mathrm{~g}, 0.57 \mathrm{mmol}), \mathrm{ZnCl}_{2}(1.0 \mathrm{M}$ in ether, $2.27 \mathrm{~mL})$ and DBU $(0.85 \mathrm{~mL}, 5.68 \mathrm{mmol})$ at $\mathrm{rt}$. To this mixture was cannulated the above Pd-catalyst solution at rt with the aid of toluene (10 $\mathrm{mL}$ ) at rt. The resulting mixture was stirred for $24 \mathrm{~h}$ at rt, heated to $60^{\circ} \mathrm{C}$ and stirred at $60^{\circ} \mathrm{C}$ for additional $12 \mathrm{~h}$. After cooling to $\mathrm{rt}$, the reaction mixture was concentrated in vacuo, diluted with minimal amount of DCM and purified by flash column chromatography (100\% hexane $\rightarrow 1: 1$ EtOAc/hexane) to give lactam $14(1.47 \mathrm{~g}, 5.38 \mathrm{mmol}, 95 \%, 87 \%$ ee $)$ as a yellow oil.

$\mathrm{R}_{\mathrm{f}}=0.3\left(\right.$ EtOAc/hexane = 1:1); ${ }^{1} \mathrm{H}$ NMR $\left(400 \mathrm{MHz}, \mathrm{CDCl}_{3}\right) \delta$ 7.34-7.23 (m, $\left.5 \mathrm{H}\right), 6.50(\mathrm{~d}, \mathrm{~J}=15.6 \mathrm{~Hz}, 1 \mathrm{H}), 6.19(\mathrm{bs}, 1 \mathrm{H})$, 6.05 (ddd, $J=15.6,8.4,6.8 \mathrm{~Hz}, 1 \mathrm{H}), 4.22(\mathrm{q}, J=7.2 \mathrm{~Hz}, 2 \mathrm{H}$ ), 2.82 (ddd, $J=13.6,6.8,1.6 \mathrm{~Hz}, 1 \mathrm{H}$ ), 2.57 (ddd, $J=13.6,8.4$, $1.2 \mathrm{~Hz}, 1 \mathrm{H}), 2.52-2.44(\mathrm{~m}, 1 \mathrm{H}), 2.41-2.37(\mathrm{~m}, 2 \mathrm{H}), 2.20-2.13(\mathrm{~m}, 1 \mathrm{H}), 1.28(\mathrm{t}, J=7.2 \mathrm{~Hz}, 3 \mathrm{H}) ;{ }^{13} \mathrm{C} \mathrm{NMR}\left(100 \mathrm{MHz}, \mathrm{CDCl}_{3}\right)$ $\delta$ 176.8, 173.2, 136.6, 135.5, 128.8, 128.0, 126.5, 122.2, 65.6, 62.0, 42.9, 30.3, 29.9, 14.4; FT-IR $\left(\mathrm{CH}_{2} \mathrm{Cl}_{2}\right) 3320,2979$, 1736, 1701, 1200, $744 \mathrm{~cm}^{-1}$; HRMS (ESI-TOF) Calcd for $\mathrm{C}_{16} \mathrm{H}_{19} \mathrm{NNaO}_{3}[\mathrm{M}+\mathrm{Na}]^{+}$296.1263, found 296.1264; $[\alpha]_{D}{ }^{25}=-31.9$ (c 1.0, $\mathrm{CHCl}_{3}$ ); HPLC (Chiralpak IA, hexane/i-PrOH $=70: 30$, flow rate $1 \mathrm{~mL} / \mathrm{min}, \lambda=254 \mathrm{~nm}$ ), $t_{\mathrm{R}}=5.89$ (major), 7.78 (minor) $\min ;$ ee $=87 \%$. 
Figure S1. HPLC chromatogram of racemic lactam 14.

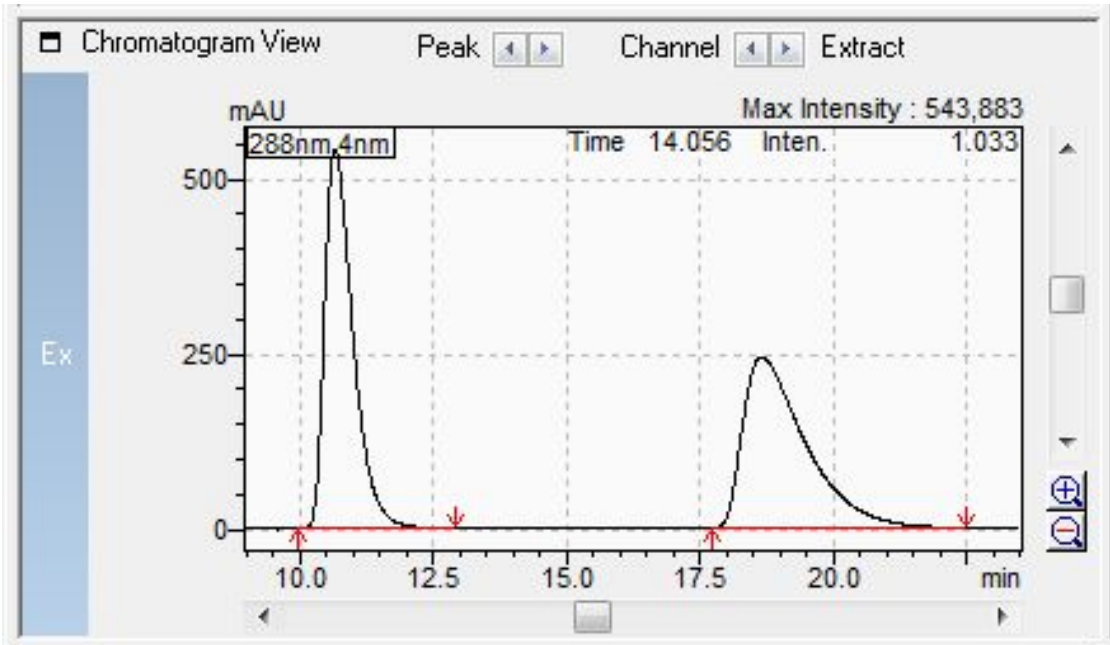

\begin{tabular}{|c|c|c|c|c|}
\hline Peak Tab & Compound & \begin{tabular}{l|l} 
Group & Calib
\end{tabular} & 1 Curve & \\
\hline Peak\# & Ret. Time & Area\% & Area & Height \\
\hline$T$ & 10.654 & 49.992 & 20133555 & 543257 \\
\hline 2 & 18.664 & 50,008 & 20140397 & 245221 \\
\hline Total & & 100,000 & 40273952 & 788478 \\
\hline
\end{tabular}

Note: HPLC trace of racemic lactam 14 (Chiralpak $O D$, hexane/i-PrOH $=80: 20$, flow rate $1 \mathrm{~mL} / \mathrm{min}$ ), $t R=18.664$ (minor), 10.654 (major) $\min$.

Figure S2. HPLC chromatogram of chiral lactam 14.

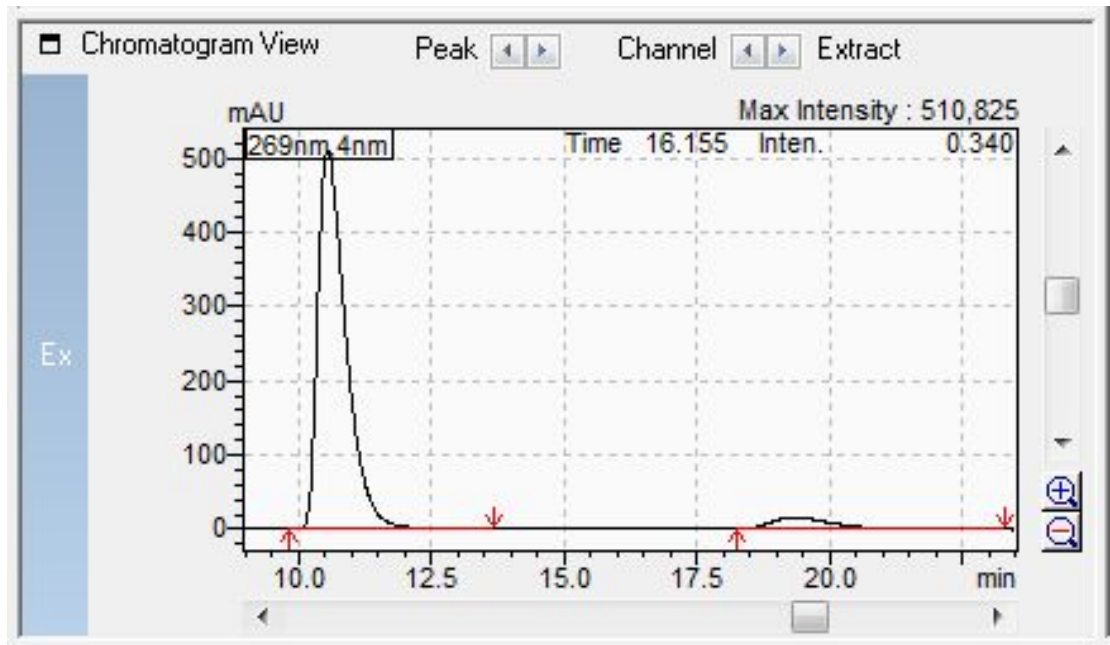

\begin{tabular}{|c|c|c|c|c|}
\hline & Compound & \begin{tabular}{l|l} 
Group & Calibr \\
\end{tabular} & Curve & \\
\hline Peak\# & Ret. Time & Area\% & Area & Height \\
\hline 1 & 10,541 & 93.345 & 18766155 & 510404 \\
\hline 2 & 19,320 & 6.655 & 1337905 & 16189 \\
\hline Total & & 100,000 & 20104060 & 526593 \\
\hline
\end{tabular}

Note: HPLC trace of enantiomerically enriched lactam 14 (Chiralpak OD, hexane/i-PrOH = 80:20, flow rate $1 \mathrm{~mL} / \mathrm{min}$ ), $t R=$ 19.320 (minor), 10.541 (major) $\min$; ee $=87 \%$. 
Ethyl (R)-2-cinnamyl-5-oxo-1-(pent-4-en-1-yl)pyrrolidine-2-carboxylate (16)
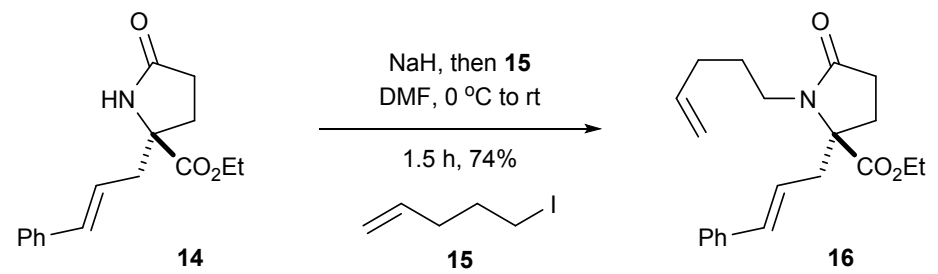

Scheme S8. N-alkylation of chiral lactam 14.

To a solution of lactam $14(2.49 \mathrm{~g}, 9.11 \mathrm{mmol})$ and 5-bromo-1-pentene $(15,2.7 \mathrm{~mL}, 22.78 \mathrm{mmol}, 2.5$ equiv. $)$ in anhydrous DMF $(15 \mathrm{~mL})$ was added $\mathrm{NaH}\left(60 \%\right.$ dispersion in mineral oil, $0.55 \mathrm{~g}, 13.66 \mathrm{mmol}, 1.5$ equiv.) at $0{ }^{\circ} \mathrm{C}$. After $10 \mathrm{~min}$ at $0{ }^{\circ} \mathrm{C}$, the reaction mixture was warmed to $\mathrm{rt}$ and stirred for additional $1 \mathrm{~h}$. The reaction mixture was then quenched with saturated $\mathrm{NH}_{4} \mathrm{Cl}(\mathrm{aq})$, and extracted with ether $(5 \times 50 \mathrm{~mL})$. The combined organic solution was washed with water $(2 \times 30 \mathrm{~mL})$, brine, dried over anhydrous $\mathrm{MgSO}_{4}$, filtered and concentrated in vacuo. The crude mixture was purified by flash column chromatography (EtOAc/hexane $=1: 4 \rightarrow 1: 2)$ to give olefin $16(2.29 \mathrm{~g}, 6.71 \mathrm{mmol}, 74 \%$ ) as a yellow oil.

$\mathrm{R}_{\mathrm{f}}=0.6\left(\right.$ EtOAc/hexane = 1:1); ${ }^{1} \mathrm{H}$ NMR $\left(400 \mathrm{MHz}, \mathrm{CDCl}_{3}\right) \delta 7.33-7.24(\mathrm{~m}, 5 \mathrm{H}), 6.54(\mathrm{~d}, J=15.6 \mathrm{~Hz}, 1 \mathrm{H}), 6.03$ (ddd, $J=$ 15.6, 8.1, $6.5 \mathrm{~Hz}, 1 \mathrm{H}$ ), 5.79 (ddt, $J=16.8,10.3,6.6 \mathrm{~Hz} 1 \mathrm{H}$ ), 5.02 (ddd, $J=17.2,3.6,1.6 \mathrm{~Hz}, 1 \mathrm{H}$ ), 4.97 (ddd, $J=10.4,3.6$, $1.2 \mathrm{~Hz}, 1 \mathrm{H}$ ), 4.21 (qd, $J=7.2,2.4 \mathrm{~Hz}, 2 \mathrm{H}$ ), $3.25(\mathrm{t}, J=8.2 \mathrm{~Hz}, 2 \mathrm{H}$ ), 2.90 (ddd, $J=14.8,8.2,1.2 \mathrm{~Hz}, 1 \mathrm{H}$ ), 2.72 (ddd, $J=$ $14.4,6.4,1.6 \mathrm{~Hz}, 1 \mathrm{H}), 2.54-2.45(\mathrm{~m}, 1 \mathrm{H}) 2.35(\mathrm{ddd}, J=18.4,10.0,3.6 \mathrm{~Hz}, 1 \mathrm{H}) 2.23(\mathrm{ddd}, J=13.6,9.8,3.6 \mathrm{~Hz}, 1 \mathrm{H}), 2.10-$ $2.02(\mathrm{~m}, 3 \mathrm{H}), 1.74-1.62(\mathrm{~m}, 2 \mathrm{H}), 1.29(\mathrm{t}, \mathrm{J}=7.2 \mathrm{~Hz}, 3 \mathrm{H}) ;{ }^{13} \mathrm{C}$ NMR $\left(100 \mathrm{MHz}, \mathrm{CDCl}_{3}\right) \delta 176.1,173.3,137.7,136.9,135.3$, 128.8, 126.3, 122.7, 115.3, 69.0, 61.9, 41.4, 38.8, 31.5, 29.8, 28.3, 27.5, 14.3; FT-IR $\left(\mathrm{CH}_{2} \mathrm{Cl}_{2}\right)$ 2977, 2930, 1732, 1696, $1398,1165,749,696 \mathrm{~cm}^{-1}$; HRMS (ESI-TOF) Calcd for $\mathrm{C}_{21} \mathrm{H}_{27} \mathrm{NNaO}_{3}[\mathrm{M}+\mathrm{Na}]^{+} 364.1889$, found 364.1890; $[\alpha]_{D} 25=+60.1(\mathrm{C}$ $\left.1.0, \mathrm{CHCl}_{3}\right)$.

\section{Ethyl $(R, Z)-3-o x o-2,3,5,6,7,10$-hexahydropyrrolo[1,2-a]azocine-10a(1H)-carboxylate (17)}

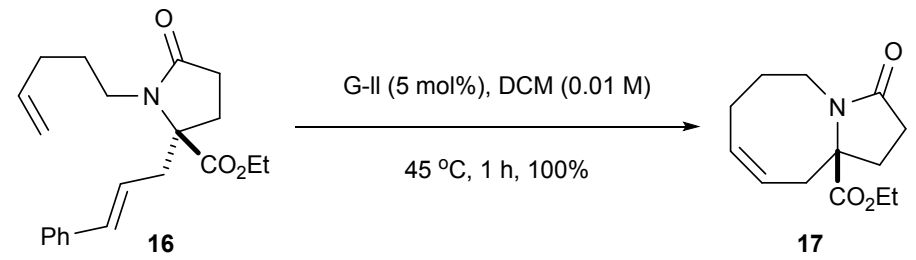

Scheme S9. Formation of aza-cyclooctene 17 via ring-closing metathesis.

To a solution of $N$-alkyl lactam $16(1.63 \mathrm{~g}, 4.77 \mathrm{mmol})$ in $\mathrm{DCM}(480 \mathrm{~mL})$ was added $2^{\text {nd }}$ generation Grubbs catalyst $(0.20 \mathrm{~g}$, $0.24 \mathrm{mmol}, 5 \mathrm{~mol} \%$ ) at rt. The reaction mixture was heated under reflux at $45^{\circ} \mathrm{C}$ for $1 \mathrm{~h}$. The reaction mixture was cooled to rt, concentrated in vacuo, and purified by flash column chromatography (EtOAc/hexane $=2: 3$ ) to give azacyclooctene 17 $(1.13 \mathrm{~g}, 4.76 \mathrm{mmol}, 100 \%)$ as a clear oil.

$\mathrm{R}_{\mathrm{f}}=0.4$ (EtOAc/hexane = 1:1); ${ }^{1} \mathrm{H}$ NMR $\left(400 \mathrm{MHz}, \mathrm{CDCl}_{3}\right) \delta 5.90(\mathrm{dt}, J=10.0,8.2 \mathrm{~Hz}, 1 \mathrm{H}), 5.68(\mathrm{dt}, J=10.0,8.2 \mathrm{~Hz}, 1 \mathrm{H})$, $4.21(\mathrm{qd}, J=7.2,0.4 \mathrm{~Hz}, 2 \mathrm{H}), 3.91(\mathrm{dt}, J=14.4,3.2 \mathrm{~Hz}, 1 \mathrm{H}), 2.77-2.68(\mathrm{~m}, 2 \mathrm{H}), 2.49(\mathrm{dd}, J=14.0,7.2 \mathrm{~Hz}, 1 \mathrm{H}), 2.39-2.35$ $(\mathrm{m}, 2 \mathrm{H}), 2.22-1.99(\mathrm{~m}, 4 \mathrm{H}), 1.96-1.89(\mathrm{~m}, 1 \mathrm{H}), 1.57-1.51(\mathrm{~m}, 1 \mathrm{H}), 1.28(\mathrm{t}, J=7.2 \mathrm{~Hz}, 1 \mathrm{H}) ;{ }^{13} \mathrm{C} \mathrm{NMR}\left(100 \mathrm{MHz}, \mathrm{CDCl}_{3}\right) \delta$ 176.1, 173.7, 135.2, 124.7, 71.7, 61.8, 42.8, 33.5, 29.6, 28.2, 27.4, 25.4, 14.3; FT-IR $\left(\mathrm{CH}_{2} \mathrm{Cl}_{2}\right) 2936,1732,1696,1398$, 1365, 1226, 1157, 1059, 749, $661 \mathrm{~cm}^{-1}$; HRMS (ESI-TOF) Calcd for $\mathrm{C}_{13} \mathrm{H}_{19} \mathrm{NNaO}_{3}[\mathrm{M}+\mathrm{Na}]^{+}$260.1263, found 260.1264; $[\alpha]_{D}^{25}=+70.4$ (c 1.0, $\mathrm{CHCl}_{3}$ ). 
<smiles>CCOC1(C)C/C=C\CCCN1C(=O)CC</smiles>

17

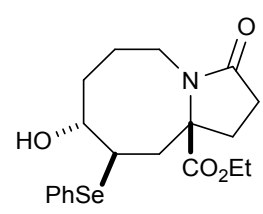

18

Scheme S10. Selenohydroxylation of 17.

To a solution of aza-cyclooctene $17(0.92 \mathrm{~g}, 3.87 \mathrm{mmol})$ in acetonitrile $(15 \mathrm{~mL})$ and water $(3 \mathrm{~mL})$ was added $\mathrm{PhSeCl}(0.74$ $\mathrm{g}, 3.87 \mathrm{mmol}, 1.0$ equiv.) at rt. The resulting mixture was stirred at $\mathrm{rt}$ for $24 \mathrm{~h}$. After completion of the reaction, the reaction mixture was quenched with saturated $\mathrm{NaHCO}_{3}(\mathrm{aq})$ and extracted with $\mathrm{DCM}$. The combined organic solution was washed with brine, dried over anhydrous $\mathrm{Na}_{2} \mathrm{SO}_{4}$, filtered and concentrated in vacuo. The crude mixture was purified by flash column chromatography (MeOH/DCM = 1:20) to give selenide $18(1.51 \mathrm{~g}, 3.68 \mathrm{mmol}, 95 \%)$ as a colorless oil.

$\mathrm{R}_{\mathrm{f}}=0.4(\mathrm{DCM} / \mathrm{MeOH}=10: 1) ;{ }^{1} \mathrm{H} \mathrm{NMR}\left(400 \mathrm{MHz}, \mathrm{CDCl}_{3}\right) \delta 7.53-7.51(\mathrm{~m}, 2 \mathrm{H}), 7.32-7.27(\mathrm{~m}, 3 \mathrm{H}), 4.26(\mathrm{qd}, J=7.2,1.8 \mathrm{~Hz}$, 2H), 4.04 (ddd, $J=8.4,8.47 .0 \mathrm{~Hz}, 1 \mathrm{H}$ ), 3.93 (ddd, $J=12.3,8.4,7.0 \mathrm{~Hz}, 1 \mathrm{H}$ ), 3.59-3.55 (m, 1H), 2.83 (ddd, $J=17.2,11.7$, $2.3 \mathrm{~Hz}, 1 \mathrm{H}), 2.6-2.45(\mathrm{~m}, 3 \mathrm{H}), 2.34(\mathrm{dd}, J=13.7,7.2 \mathrm{~Hz}, 1 \mathrm{H}), 2.18(\mathrm{ddd}, J=15.2,11.8,1.6 \mathrm{~Hz}, 1 \mathrm{H}), 2.11-2.06(\mathrm{~m}, 1 \mathrm{H})$, 2.02-1.89 (m, 2H), 1.84-1.79 (m, 2H), $1.32(\mathrm{t}, J=7.2 \mathrm{~Hz}, 3 \mathrm{H}) ;{ }^{13} \mathrm{C} \mathrm{NMR}\left(100 \mathrm{MHz}, \mathrm{CDCl}_{3}\right) \delta 175.3,169.8,133.6,129.6$, 129.1, 128.1, 73.1, 67.5, 62.1, 52.6, 41.2, 40.9, 32.7, 32.2, 30.2, 26.2, 14.2; FT-IR $\left(\mathrm{CH}_{2} \mathrm{Cl}_{2}\right) 2975,2938,1730,1577,1477$, $1255,1198,1096,1022,738,692 \mathrm{~cm}^{-1}$; HRMS (ESI-TOF) Calcd for $\mathrm{C}_{19} \mathrm{H}_{26} \mathrm{NO}_{4} \mathrm{Se}[\mathrm{M}+\mathrm{H}]^{+} 412.1027$, found 412.1027; $[\alpha]_{\mathrm{D}}{ }^{25}$ $=-14.8\left(\mathrm{c} 1.0, \mathrm{CHCl}_{3}\right)$.

Figure S3. Stereochemical assignment of 18.

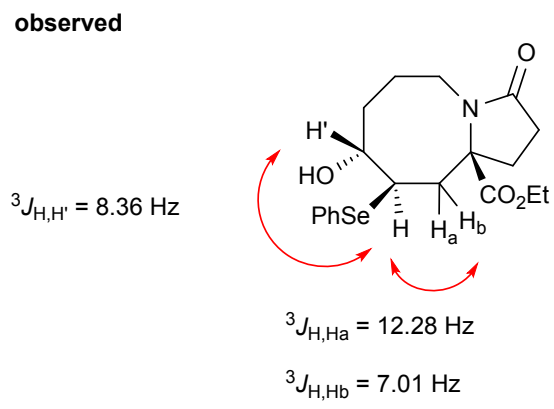

observed ${ }^{3} \mathrm{~J}_{\mathrm{H}, \mathrm{H}^{\prime}}$ value $(8.36 \mathrm{~Hz})$ is closer to that of 18 than 18'

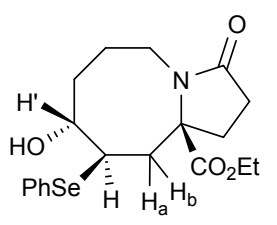

18<smiles>CCO[C@]1(C)CCC(=O)N1CCC[C@H](O)C([18OH])[18OH]</smiles>

18 '

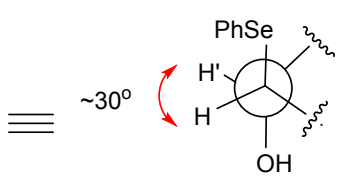

estimated

$$
{ }^{3} J_{\mathrm{H}, \mathrm{H}^{\prime}} \sim 11 \mathrm{~Hz}
$$

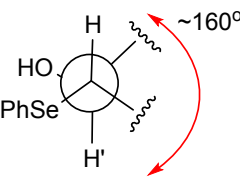

estimated

$3^{3} J_{\mathrm{H}, \mathrm{H}^{\prime}} \sim 14 \mathrm{~Hz}$

\section{Ethyl (10aR,Z)-8-hydroxy-3-oxo-2,3,5,6,7,8-hexahydropyrrolo[1,2-a]azocine-10a(1H)-carboxylate (19)}

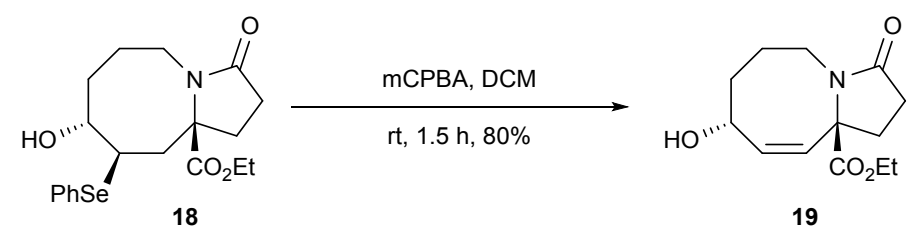

Scheme S11. Oxidative elimination of phenyl selenide 18.

To a solution of selenide $18(1.46 \mathrm{~g}, 3.56 \mathrm{mmol})$ in $\mathrm{DCM}(50 \mathrm{~mL})$ was added $\mathrm{mCPBA}(77 \%, 0.96 \mathrm{~g}, 4.27 \mathrm{mmol})$ at rt. The resulting mixture was stirred at $\mathrm{rt}$ for $1.5 \mathrm{~h}$. Then, the reaction mixture was concentrated in vacuo and purified by flash column chromatography $(\mathrm{MeOH} / \mathrm{DCM}=1: 20)$ to give allyl alcohol $19(0.72 \mathrm{~g}, 2.84 \mathrm{mmol}, 80 \%)$ as a colorless oil.

$\mathrm{R}_{\mathrm{f}}=0.3(\mathrm{DCM} / \mathrm{MeOH}=10: 1) ;{ }^{1} \mathrm{H} \mathrm{NMR}\left(400 \mathrm{MHz}, \mathrm{CDCl}_{3}\right) \delta 6.04(\mathrm{dd}, J=6.0,1.6 \mathrm{~Hz}, 1 \mathrm{H}), 5.68(\mathrm{dd}, J=6.0,2.4 \mathrm{~Hz}, 1 \mathrm{H})$, 4.43 (tt, $J=7.9,2 \mathrm{~Hz} 1 \mathrm{H}$ ), 4.25 (q, $J=7.2 \mathrm{~Hz}, 2 \mathrm{H}$ ), 3.35 (ddd, $J=10.4,6.8,3.2 \mathrm{~Hz}, 1 \mathrm{H}$ ), 2.71-2.62 (m, 2H), 2.50 (ddd, $J=$ 
16.8, 9.6, 1.2 Hz, 1H), 2.38 (ddd, $J=15.2,9.6,1.2 \mathrm{~Hz}, 1 \mathrm{H}), 2.17-2.11(\mathrm{~m}, 1 \mathrm{H}), 2.02-1.94(\mathrm{~m}, 1 \mathrm{H}), 1.94-1.82(\mathrm{~m}, 2 \mathrm{H}), 1.70-$ $1.62(\mathrm{~m}, 1 \mathrm{H}), 1.32(\mathrm{t}, J=7.2 \mathrm{~Hz}, 3 \mathrm{H}) ;{ }^{13} \mathrm{C} \mathrm{NMR}\left(100 \mathrm{MHz}, \mathrm{CDCl}_{3}\right) \delta 175.5,169.6,143.8,129.5,79.6,71.9,62.1,50.4,32.3$, 31.9, 30.1, 26.6, 14.2; FT-IR $\left(\mathrm{CH}_{2} \mathrm{Cl}_{2}\right) 3361,2967,2920,1730,1447,1369,1239,1108,1022,706 \mathrm{~cm}^{-1}$; HRMS (ESI-TOF) Calcd for $\mathrm{C}_{13} \mathrm{H}_{19} \mathrm{NNaO}_{4}[\mathrm{M}+\mathrm{Na}]^{+} 276.1212$, found 276.1212; $[\alpha]_{\mathrm{D}}^{25}=+2.9$ (c 1.0, $\mathrm{CHCl}_{3}$ ).

Figure S4. Stereochemical assignment of 19.

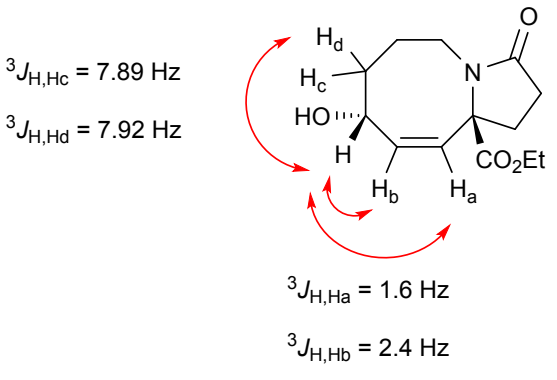

observed small $\Delta^{3} J(0.03 \mathrm{~Hz})$ value is closer to that of 19 than $19-\alpha-\mathrm{OH}$<smiles></smiles>

19

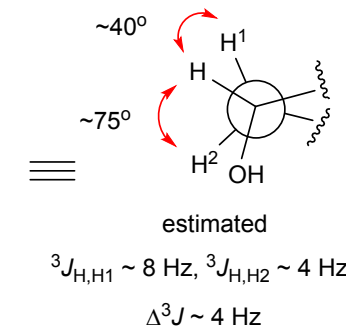<smiles>CCO[C@@]12/C=C/[C@H](O)[C@H](O)CCCN1C(=O)CC2</smiles>

${ }^{3} J_{\mathrm{H}, \mathrm{H} 1} \sim 14 \mathrm{~Hz},{ }^{3} J_{\mathrm{H}, \mathrm{H} 2} \sim 6 \mathrm{~Hz}$ $\Delta^{3} \mathrm{~J} \sim 8 \mathrm{~Hz}$

Ethyl (10aR,Z)-8-bromo-3-oxo-2,3,5,6,7,8-hexahydropyrrolo[1,2-a]azocine-10a(1H)-carboxylate (20)<smiles>CCOC1(CC)C2CCC(=O)N1CCC[C@H](O)CC2</smiles>

$(+)-19$

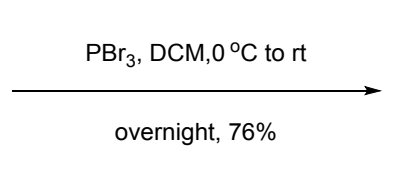

Scheme S12. Bromination of allylic alcohol (+)-19<smiles>CCOC12C=CC(Br)CCCN1C(=O)CC2</smiles>

$(+)-20$

To a solution of alcohol $(+)-19(377 \mathrm{mg}, 1.49 \mathrm{mmol})$ in DCM $(5 \mathrm{~mL})$ was added $\mathrm{PBr}_{3}(0.98 \mathrm{~mL}, 1.04 \mathrm{mmol})$ at $0{ }^{\circ} \mathrm{C}$. The resulting solution was slowly warmed to rt and stirred for $6 \mathrm{~h}$. After completion of reaction, ice-water was added and the resulting mixture was extracted with DCM several times. The combined organic solution was washed with saturated $\mathrm{NaHCO}_{3}(\mathrm{aq})$, brine, dried over anhydrous $\mathrm{Na}_{2} \mathrm{SO}_{4}$, filtered and concentrated in vacuo. The crude mixture was purified by flash column chromatography (EtOAc/hexane $=1: 2)$ to give allyl bromide $(+)-20(359 \mathrm{mg}, 1.13 \mathrm{mmol}, 76 \%)$ as a colorless oil.

$\mathrm{R}_{\mathrm{f}}=0.2\left(\right.$ EtOAc/hexane = 1:2); ${ }^{1} \mathrm{H}$ NMR $\left(400 \mathrm{MHz}, \mathrm{CDCl}_{3}\right) \delta 5.94(\mathrm{~m}, 2 \mathrm{H}), 5.06$ (ddd, $\left.\mathrm{J}=11.6,5.9,3.6 \mathrm{~Hz}, 1 \mathrm{H}\right), 4.24$ (qd, $J=7.2,1.2 \mathrm{~Hz}, 2 \mathrm{H}$ ), 3.95 (ddd, $J=14.1,9.2,2.8 \mathrm{~Hz}, 1 \mathrm{H}$ ), 2.98 (ddd, $J=14.1,8.0,2.4 \mathrm{~Hz}, 1 \mathrm{H}) 2.48-2.40(\mathrm{~m}, 3 \mathrm{H}), 2.37-2.32$ $(\mathrm{m}, 1 \mathrm{H}), 2.15-1.91(\mathrm{~m}, 3 \mathrm{H}), 1.78-1.74(\mathrm{~m}, 1 \mathrm{H}), 1.29(\mathrm{t}, J=7.2 \mathrm{~Hz}, 3 \mathrm{H}) ;{ }^{13} \mathrm{C} \mathrm{NMR}\left(100 \mathrm{MHz}, \mathrm{CDCl}_{3}\right) \delta 175.8,171.8,133.9$ 128.7, 70.1, 62.4, 46.5, 41.9, 38.9, 33.6, 29.8, 25.2, 14.3; FT-IR $\left(\mathrm{CH}_{2} \mathrm{Cl}_{2}\right)$ 3357, 2922, 2851, 1734, 1700, 1445, 1388, 1353, 1241, 1171, 1053, 912, $740 \mathrm{~cm}^{-1}$; HRMS (ESI-TOF) Calcd for $\mathrm{C}_{13} \mathrm{H}_{18} \mathrm{NNaO}_{3} \mathrm{Br}[\mathrm{M}+\mathrm{Na}]^{+} 338.0368$, found 338.0366; $[\alpha]_{D}{ }^{25}=$ $+88.6\left(\mathrm{c} 0.75, \mathrm{CHCl}_{3}\right)$. 
(10aR,Z)-10a-((tert-Butyldimethylsilyl)oxy)methyl)-8-((2,2,6,6-tetramethylpiperidin-1-yl)oxy)-1,5,6,7,8,10ahexahydropyrrolo[1,2-a]azocin-3(2H)-one (22)<smiles>C=CC1(OCC)/C=C\C(Br)CCCN1C(=O)CC</smiles>

20

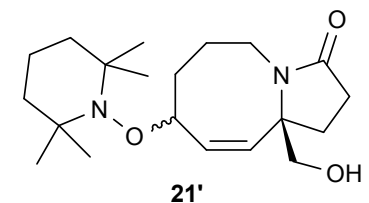

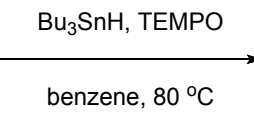

benzene, 8

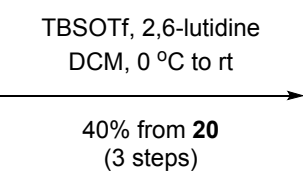

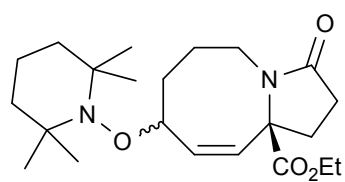

21

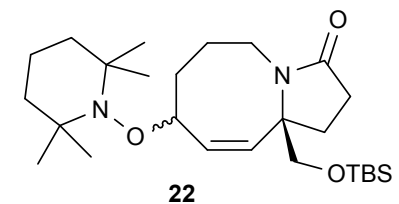

Scheme S13. Formation of TBS ether 22.

To a solution of bromide $(+)-20(240 \mathrm{mg}, 0.76 \mathrm{mmol})$ in benzene $(10 \mathrm{~mL})$ were added TEMPO $(238 \mathrm{mg}, 1.52 \mathrm{mmol}, 2.0$ equiv.) and tributyltin hydride $(0.33 \mathrm{~mL}, 1.22 \mathrm{mmol}, 1.6$ equiv.). The resulting mixture was heated under reflux for $30 \mathrm{~min}$. To this mixture were added additional TEMPO (238 mg, 2.0 equiv.) and tributyltin hydride $(0.33 \mathrm{~mL}, 1.6$ equiv.) simultaneously every $30 \mathrm{~min}$ for $2 \mathrm{~h}$ under reflux condition. After $20 \mathrm{~min}$, the reaction mixture was cooled to rt, concentrated in vacuo and purified by flash chromatography on silica (EtOAc/hexane $=1: 4$ ) to afford oxime ether $\mathbf{2 1}$, which was used directly in the next step. To a solution of oxime ether 21 in THF $(11 \mathrm{~mL})$ at $-78{ }^{\circ} \mathrm{C}$ was added $\mathrm{LiAlH}_{4}(2.28 \mathrm{~mL}, 1 \mathrm{M}$ in THF, 3.0 equiv.). The resulting mixture was stirred at $-78{ }^{\circ} \mathrm{C}$ for $1.5 \mathrm{~h}$ and warm to $0{ }^{\circ} \mathrm{C}$. After $15 \mathrm{~min}$ at $0{ }^{\circ} \mathrm{C}$, the reaction mixture was quenched by addition of 5 drops of $\mathrm{H}_{2} \mathrm{O}$, and filtered through a pad of Celite with the aid of ether. After concentration in vacuo, crude alcohol 21' was used directly in the next step without further purification. To a solution of alcohol 21' in $\operatorname{DCM}(7.6 \mathrm{~mL})$ were added 2,6-lutidine $(0.26 \mathrm{~mL}, 3.0$ equiv. $)$ and TBSOTf $(0.35 \mathrm{~mL}, 2.0$ equiv. $)$ at $0{ }^{\circ} \mathrm{C}$. The mixture was warmed to rt and stirred overnight. The reaction mixture was then quenched with saturated aqueous $\mathrm{NaHCO}_{3}$ solution and extracted with DCM. The separated organic layer was dried over $\mathrm{MgSO}_{4}$, concentrated and purified by flash chromatography on silica (EtOAc/hexane $=1: 5)$ to afford TBS ether $22(140 \mathrm{mg}, 0.3 \mathrm{mmol})$ in $40 \%$ yield from bromide 20 over three steps.

$\mathrm{R}_{\mathrm{f}}=0.32($ EtOAc/hexane $=1: 4) ;{ }^{1} \mathrm{H} \mathrm{NMR}\left(400 \mathrm{MHz}, \mathrm{CDCl}_{3}\right) \delta 5.87(\mathrm{dd}, J=13.3,7.6 \mathrm{~Hz}, 1 \mathrm{H}), 5.38(\mathrm{~d}, J=13.4 \mathrm{~Hz}, 1 \mathrm{H})$, $4.47(\mathrm{t}, J=7.9 \mathrm{~Hz}, 1 \mathrm{H}), 3.72(\mathrm{dt}, J=13.8,4.0 \mathrm{~Hz}, 1 \mathrm{H}), 3.55(\mathrm{~d}, J=10.1 \mathrm{~Hz}, 1 \mathrm{H}), 3.41(\mathrm{~d}, J=10.1 \mathrm{~Hz}, 1 \mathrm{H}), 2.68(\mathrm{ddd}, J=$ 13.3, 11.7, $2.2 \mathrm{~Hz}, 1 \mathrm{H}), 2.71-2.64(\mathrm{~m}, 1 \mathrm{H}), 2.50-2.26(\mathrm{~m}, 3 \mathrm{H}), 2.19-2.00(\mathrm{~m}, 3 \mathrm{H}), 1.62-1.50(\mathrm{~m}, 2 \mathrm{H}), 1.48-1.35(\mathrm{~m}, 4 \mathrm{H})$, 1.34-1.21 (m, 2H), 1.21-0.98 (m, 12H), $0.87(\mathrm{~s}, 9 \mathrm{H}), 0.03(\mathrm{~s}, 3 \mathrm{H}), 0.02(\mathrm{~m}, 3 \mathrm{H}) ;{ }^{13} \mathrm{C}$ NMR $\left(100 \mathrm{MHz}, \mathrm{CDCl}_{3}\right) \delta 176.4,134.1$, 130.5, 83.0, 67.7, 67.3, 60.4, 59.9, 41.3, 41.2, 40.4, 34.9, 34.5, 30.9, 30.4, 30.3, 26.3, 23.3, 21.0, 18.7, 17.7, -5.02, -5.06; FT-IR $\left(\mathrm{CH}_{2} \mathrm{Cl}_{2}\right) 2928,2857,1692,1361,1257,1112,959,839,780 \mathrm{~cm}^{-1} ; \mathrm{HRMS}$ (ESI-TOF) Calcd for $\mathrm{C}_{26} \mathrm{H}_{49} \mathrm{~N}_{2} \mathrm{O}_{3} \mathrm{Si}[\mathrm{M}+\mathrm{H}]+$ 465.3512, found 465.3512; $[\alpha]_{D}{ }^{25}=+74.8\left(\mathrm{c} 1.0, \mathrm{CHCl}_{3}\right)$.

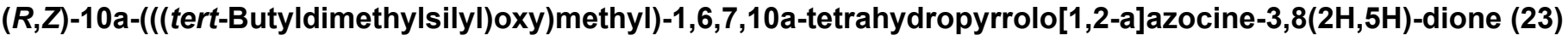

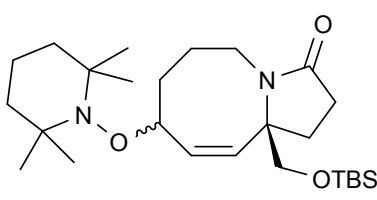

22

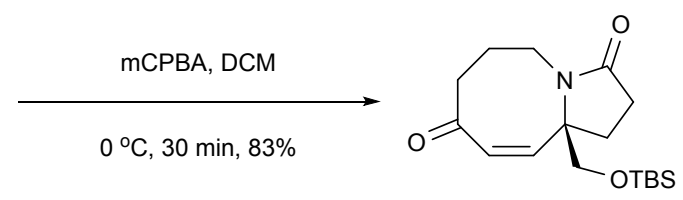

23

Scheme S14. Formation of enone 23.

To a solution of TBS ether 22 (93 mg, $0.2 \mathrm{mmol})$ in DCM (20 mL) was added mCPBA (63 mg, 1.4 equiv.) at $0{ }^{\circ} \mathrm{C}$. After 30 min at $0{ }^{\circ} \mathrm{C}$, the reaction mixture was quenched with saturated aqueous $\mathrm{Na}_{2} \mathrm{~S}_{2} \mathrm{O}_{3}$ and extracted with $\mathrm{DCM}$ several times. The combined organic solution was dried over anhydrous $\mathrm{MgSO}_{4}$, filtered, concentrated in vacuo and purified by silica-gel column chromatography (EtOAc/hexane $=1: 2)$ to give the corresponding cyclic enone $23(55 \mathrm{mg}, 0.17 \mathrm{mmol})$ in $83 \%$ yield.

$\mathrm{R}_{\mathrm{f}}=0.26($ EtOAc/hexane $=1: 1) ;{ }^{1} \mathrm{H} \mathrm{NMR}\left(400 \mathrm{MHz}, \mathrm{CDCl}_{3}, 55^{\circ} \mathrm{C}\right) \delta 5.77(\mathrm{~s}, 2 \mathrm{H}), 4.00-4.08(\mathrm{~m}, 1 \mathrm{H}), 3.79(\mathrm{~d}, \mathrm{~J}=10.6 \mathrm{~Hz}$, $1 \mathrm{H}), 3.65(\mathrm{~d}, J=10.6 \mathrm{~Hz}, 1 \mathrm{H}), 2.05-3.05(\mathrm{~m}, 1 \mathrm{H}), 2.50-2.23(\mathrm{~m}, 4 \mathrm{H}), 2.18$ (dddd, $J=16.6,9.2,2.6,0.7 \mathrm{~Hz}, 1 \mathrm{H}$ ), 1.94 (ddd, $J=12.4,10.9,9.2 \mathrm{~Hz}, 1 \mathrm{H}), 1.90-1.80(\mathrm{~m}, 1 \mathrm{H}), 1.73(\mathrm{ddd}, J=11.8,9.0,2.6 \mathrm{~Hz}, 1 \mathrm{H}), 0.90(\mathrm{~s}, 9 \mathrm{H}), 0.09(\mathrm{~s}, 6 \mathrm{H}) ;{ }^{13} \mathrm{C} \mathrm{NMR}$ 
$\left(100 \mathrm{MHz}, \mathrm{CDCl}_{3}\right) \delta 209.2,176.3,137.2,127.3,67.9,66.1,41.1,39.2,29.4,28.4,25.8,23.9,18.3,-5.5 ; \mathrm{FT}-\mathrm{IR}\left(\mathrm{CH}_{2} \mathrm{Cl}_{2}\right)$ 3357, 3200, 2926, 2854, 1698, 1469, 1400, 1253, 1114, 1071, 841, $777 \mathrm{~cm}^{-1}$; HRMS (ESI-TOF) Calcd for $\mathrm{C}_{17} \mathrm{H}_{29} \mathrm{NNaO}_{3} \mathrm{Si}$ $[\mathrm{M}+\mathrm{Na}]^{+} 346.1814$, found $346.1815 ;[\alpha]_{\mathrm{D}}^{25}=+84.9\left(\mathrm{c} 1.0, \mathrm{CHCl}_{3}\right)$.

\section{$(R, 7 E, 9 Z)-10 a-(((t e r t-B u t y l d i m e t h y l s i l y l) o x y)$ methyl)-3-oxo-1,2,3,5,6,10a-hexahydropyrrolo[1,2-a]azocin-8-yl trifluoromethanesulfonate (24)}<smiles>O=C1C=CC2(CCCCCC2)CCC1=O</smiles>

23

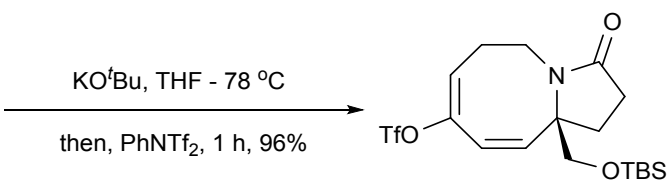

24

Scheme S15. Triflation of enone 23.

To a solution of enone $23(229 \mathrm{mg}, 0.7 \mathrm{mmol})$ in THF (4.6 mL) was added KOtBu (1.2 mL, $1 \mathrm{M} \mathrm{in} \mathrm{THF,} 1.5$ equiv.) at $-78^{\circ} \mathrm{C}$. The resulting mixture was stirred for $30 \mathrm{~min}$ at $-78{ }^{\circ} \mathrm{C}$ and cannulated with a solution of $\mathrm{PhNTf}_{2}$ (383 mg, 1.5 equiv.) in THF $(4.6 \mathrm{~mL})$ at $-78{ }^{\circ} \mathrm{C}$. After $30 \mathrm{~min}$ at $-78^{\circ} \mathrm{C}$, the reaction mixture was quenched with saturated aqueous $\mathrm{NH}_{4} \mathrm{Cl}$ solution and extracted with EtOAc several times. The combined organic solution was washed with brine, dried over anhydrous $\mathrm{MgSO}_{4}$, filtered, concentrated in vacuo and purified by silica-gel column chromatography (EtOAc/hexane $=1: 5)$ to give the corresponding vinyl triflate 24 (310 $\mathrm{mg}, 0.68 \mathrm{mmol})$ in $96 \%$ yield.

$\mathrm{R}_{\mathrm{f}}=0.18\left(\right.$ EtOAc/hexane = 1:4); ${ }^{1} \mathrm{H}$ NMR $\left(400 \mathrm{MHz}, \mathrm{CDCl}_{3}\right) \delta 5.79(\mathrm{t}, J=8.6 \mathrm{~Hz}, 1 \mathrm{H}), 5.73(\mathrm{~d}, J=12.5 \mathrm{~Hz}, 1 \mathrm{H}), 5.57(\mathrm{~d}, J$ $=12.5 \mathrm{~Hz}, 1 \mathrm{H}), 3.96(\mathrm{td}, J=13.6,4.5 \mathrm{~Hz}, 1 \mathrm{H}), 3.77(\mathrm{~d}, J=10.6 \mathrm{~Hz}, 1 \mathrm{H}), 3.63(\mathrm{~d}, J=10.6 \mathrm{~Hz}, 1 \mathrm{H}), 3.00(\mathrm{dd}, J=13.5,5.5$ $\mathrm{Hz}, 1 \mathrm{H}), 2.55-2.39(\mathrm{~m}, 2 \mathrm{H}), 2.32-2.23(\mathrm{~m}, 2 \mathrm{H}), 1.98-1.80(\mathrm{~m}, 2 \mathrm{H}), 0.88(\mathrm{~s}, 9 \mathrm{H}), 0.08(\mathrm{~s}, 6 \mathrm{H}) ;{ }^{13} \mathrm{C} \mathrm{NMR}\left(100 \mathrm{MHz}, \mathrm{CDCl}_{3}\right) \delta$ 175.8, 147.2, 137.4, 120.2, 119.4, 118.8, 117.0, 68.3, 68.2, 36.2, 30.5, 28.5, 25.9, 25.1, 18.3, -5.47, -5.48; FT-IR $\left(\mathrm{CH}_{2} \mathrm{Cl}_{2}\right)$ 3357, 2955, 2928, 2855, 1700, 1418, 1212, 1142, 10124, 922, 841, 780, $614 \mathrm{~cm}^{-1}$; HRMS (ESI-TOF) Calcd for $\mathrm{C}_{18} \mathrm{H}_{28} \mathrm{NNaO}_{5} \mathrm{~F}_{3} \mathrm{SiS}[\mathrm{M}+\mathrm{Na}]^{+} 478.1307$, found $478.1310 ;[\alpha]_{D}^{25}=+158.3\left(\mathrm{c} 1.0, \mathrm{CHCl}_{3}\right.$ ).

$(R, Z)-13 a-(($ tert-Butyldimethylsilyl)oxy)methyl)-8-methoxy-1,2,5,6,11,13a-hexahydro-3Hpyrrolo[1',2':1,8]azocino[5,4-b]indol-3-one (27)

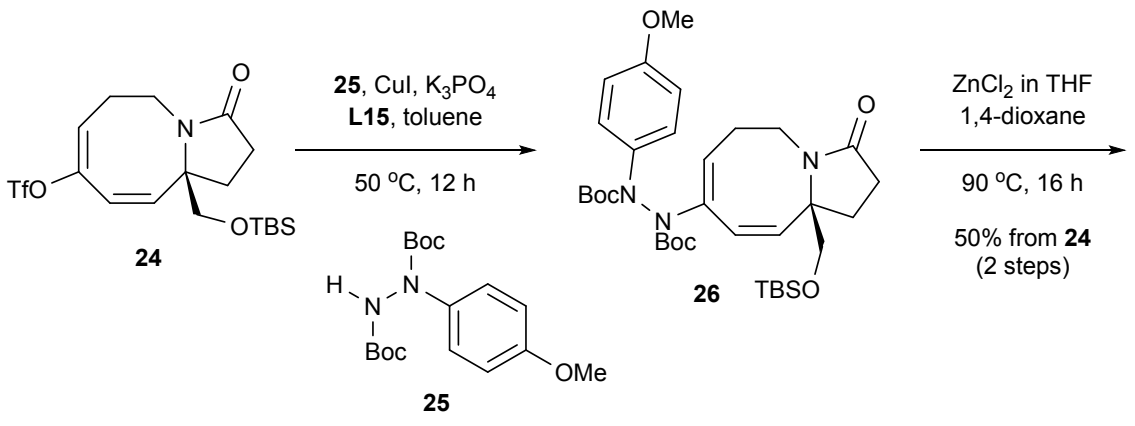

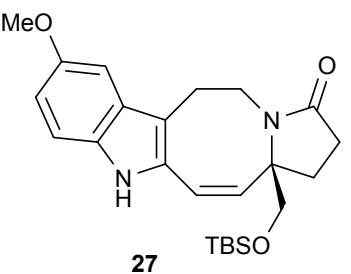

Scheme S16. Formation of pyrroloazocine indole 27 via copper-mediated $\mathrm{C}-\mathrm{N}$ coupling reaction and indolization.

To a solution of vinyl triflate $24(137 \mathrm{mg}, 0.3 \mathrm{mmol})$ in toluene $(0.6 \mathrm{~mL})$ were added aryl hydrazine $25(122 \mathrm{mg}, 0.36 \mathrm{mmol}$, 1.2 equiv.), $\mathrm{Cul}$ ( $57 \mathrm{mg}, 0.3 \mathrm{mmol}, 1.0$ equiv.) and $\mathrm{K}_{3} \mathrm{PO}_{4}(128 \mathrm{mg}, 0.6 \mathrm{mmol}, 2.0$ equiv.) at rt. After $30 \mathrm{~min}$ at rt under $\mathrm{Ar}$ atmosphere, the reaction mixture was treated with $\mathbf{L} 15(0.07 \mathrm{~mL}, 0.6 \mathrm{mmol}, 2.0$ equiv.) at rt. The resulting mixture was heated at $90{ }^{\circ} \mathrm{C}$ for $12 \mathrm{~h}$, cooled to rt, filtered through a pad of silica gel with the aid of EtOAc, concentrated in vacuo, and purified by flash column chromatography (EtOAc/heaxane $=1: 4$ ) to afford the corresponding ene-hydrazine 26, which was used directly in the next step. To a solution of ene-hydrazine 26 in 1,4-dioxane $(3.0 \mathrm{~mL})$ was added $\mathrm{ZnCl}_{2}(1.4 \mathrm{~mL}, 0.5 \mathrm{M}$ in THF, 2.2 equiv.) at rt. The resulting mixture was heated under reflux at $90^{\circ} \mathrm{C}$ for $16 \mathrm{~h}$, cooled to rt, quenched with saturated aqueous $\mathrm{NaHCO}_{3}(1.0 \mathrm{~mL})$ and $\mathrm{NH}_{4} \mathrm{Cl}(1.0 \mathrm{~mL})$ and extracted with $\mathrm{DCM}$ several times. The combined organic solution was dried over $\mathrm{MgSO}_{4}$, concentrated in vacuo and purified by flash chromatography on silica (EtOAc/hexane = $1: 1)$ to afford indole 27 ( $65 \mathrm{mg}, 0.15 \mathrm{mmol})$ in $50 \%$ yield.

$\mathrm{R}_{\mathrm{f}}=0.3(\mathrm{EtOAc} / \mathrm{hexane}=1: 1) ;{ }^{1} \mathrm{H} \mathrm{NMR}\left(400 \mathrm{MHz}, \mathrm{CDCl}_{3}\right) \delta 7.90(\mathrm{~s}, 1 \mathrm{H}), 7.07(\mathrm{~d}, J=8.7 \mathrm{~Hz}, 1 \mathrm{H}), 6.96(\mathrm{~d}, J=2.3 \mathrm{~Hz}, 1 \mathrm{H})$, 
6.77 (dd, $J=8.8,2.4 \mathrm{~Hz}, 1 \mathrm{H}), 6.33(\mathrm{~d}, J=12.5 \mathrm{~Hz}, 1 \mathrm{H}), 5.42$ (d, $J=12.4 \mathrm{~Hz}, 1 \mathrm{H}), 4.09$ (td, $J=12.8,4.6 \mathrm{~Hz}, 1 \mathrm{H}), 3.90(\mathrm{~d}$, $J=10.6 \mathrm{~Hz}, 1 \mathrm{H}), 3.84(\mathrm{~s}, 3 \mathrm{H}), 3.70(\mathrm{~d}, J=10.5,1 \mathrm{H}), 3.24-3.07(\mathrm{~m}, 2 \mathrm{H}), 2.82(\mathrm{ddd}, J=14.9,13.0,5.6,1 \mathrm{H}), 2.30-2.05(\mathrm{~m}$, $3 \mathrm{H}), 1.85-1.76(\mathrm{~m}, 1 \mathrm{H}), 0.92(\mathrm{~s}, 9 \mathrm{H}), 0.12(\mathrm{~s}, 6 \mathrm{H}) ;{ }^{13} \mathrm{C} \mathrm{NMR}\left(100 \mathrm{MHz}, \mathrm{CDCl}_{3}\right) \delta 175.5,154.2,132.5,131.5,131.3,128.1$, 119.1, 112.9, 111.5, 111.0, 100.2, 68.7, 67.1, 55.9, 38.3, 31.3, 29.1, 25.9, 22.6, 18.4, -5.4; FT-IR $\left(\mathrm{CH}_{2} \mathrm{Cl}_{2}\right) 3263,2953$, 2928, 2854, 1673, 1465, 1404, 1257, 1218, 1112, 839, $777 \mathrm{~cm}^{-1}$; HRMS (ESI-TOF) Calcd for $\mathrm{C}_{24} \mathrm{H}_{34} \mathrm{~N}_{2} \mathrm{O}_{3} \mathrm{SiNa}$ [M+Na] 449.2236, found 449.2238; $[\alpha]_{\mathrm{D}}{ }^{25}=+131.9\left(\mathrm{c} 1.0, \mathrm{CHCl}_{3}\right)$.

\section{Methyl $(R, Z)-13 a-(($ tert-butyldimethylsilyl)oxy)methyl)-8-methoxy-3-oxo-1,2,3,5,6,13a-hexahydro-11H- pyrrolo[1',2':1,8]azocino[5,4-b]indole-11-carboxylate (28)}
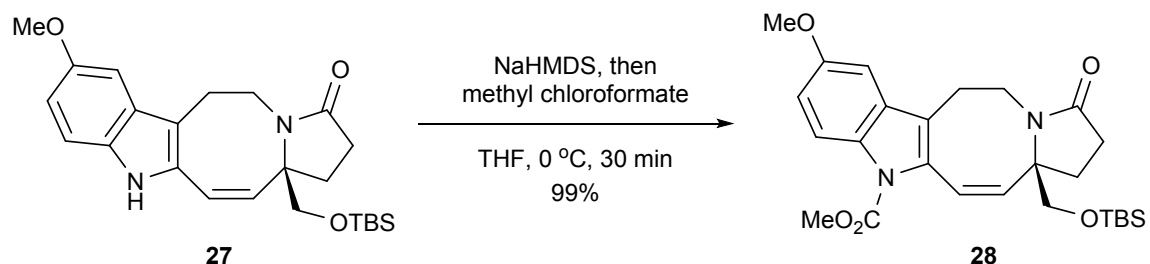

Scheme S17. Introduction of methyl carbamate 28.

To a solution of indole $27(26 \mathrm{mg}, 0.06 \mathrm{mmol})$ in THF $(1.3 \mathrm{~mL})$ was added NaHMDS $(0.09 \mathrm{~mL}, 1 \mathrm{M}$ in THF, 1.5 equiv.) at 0 ${ }^{\circ} \mathrm{C}$. The resulting mixture was stirred for $30 \mathrm{~min}$ at $0{ }^{\circ} \mathrm{C}$ and added with methyl chloroformate $(0.02 \mathrm{~mL}, 4.0$ equiv. $)$ at $0{ }^{\circ} \mathrm{C}$. After $30 \mathrm{~min}$ at $0{ }^{\circ} \mathrm{C}$, the reaction mixture was quenched with saturated $\mathrm{NH}_{4} \mathrm{Cl}(\mathrm{aq})$ and extracted with EtOAc several times. The combined organic solution was washed with brine, dried over anhydrous $\mathrm{MgSO}_{4}$, filtered, concentrated in vacuo and purified by silica-gel column chromatography (EtOAc/hexane =1:2) to give carbamate $28(30 \mathrm{mg}, 0.06 \mathrm{mmol})$ in $99 \%$ yield.

$\mathrm{R}_{\mathrm{f}}=0.5$ (EtOAc/hexane = 1:1); ${ }^{1} \mathrm{H}$ NMR $\left(400 \mathrm{MHz}, \mathrm{CDCl}_{3}\right) \delta 7.87(\mathrm{~d}, \mathrm{~J}=9.0 \mathrm{~Hz}, 1 \mathrm{H}), 6.94(\mathrm{~d}, \mathrm{~J}=2.5 \mathrm{~Hz}, 1 \mathrm{H}), 6.86(\mathrm{dd}, \mathrm{J}$ $=9.0,2.5 \mathrm{~Hz}, 1 \mathrm{H}), 6.62(\mathrm{~d}, J=12.4 \mathrm{~Hz}, 1 \mathrm{H}), 5.46(\mathrm{~d}, J=12.4 \mathrm{~Hz}, 1 \mathrm{H}), 4.00(\mathrm{td}, \mathrm{J}=13.1,4.9 \mathrm{~Hz}, 1 \mathrm{H}), 3.99(\mathrm{~s}, 3 \mathrm{H}), 3.89(\mathrm{~d}$, $J=10.6 \mathrm{~Hz}, 1 \mathrm{H}), 3.86(\mathrm{~s}, 3 \mathrm{H}), 3.71(\mathrm{~d}, J=10.6 \mathrm{~Hz}, 1 \mathrm{H}), 3.10(\mathrm{dd}, J=13.2,5.3 \mathrm{~Hz}, 1 \mathrm{H}), 2.95(\mathrm{dd}, J=13.9,3.5 \mathrm{~Hz}, 1 \mathrm{H})$, $2.79(\mathrm{td}, J=13.6,5.6 \mathrm{~Hz}, 1 \mathrm{H}), 2.32$ (ddd, $J=16.1,11.7,8.6 \mathrm{~Hz}, 1 \mathrm{H}), 2.12(\mathrm{dd}, J=16.0,8.3 \mathrm{~Hz}, 1 \mathrm{H}), 2.07-1.94(\mathrm{~m}, 1 \mathrm{H})$, 1.85 (ddd, $J=11.0,7.6,1.0 \mathrm{~Hz}, 1 \mathrm{H}), 0.92(\mathrm{~s}, 9 \mathrm{H}), 0.12(\mathrm{~s}, 6 \mathrm{H}) ;{ }^{13} \mathrm{C} \mathrm{NMR}\left(100 \mathrm{MHz}, \mathrm{CDCl}_{3}\right) \delta 175.6,156.3,152.5,134.4$, 130.6, 130.0, 129.1, 120.6, 117.8, 116.6, 113.5, 101.0, 68.2, 67.8, 55.7, 53.5, 37.1, 31.1, 28.9, 25.9, 22.2, 18.3, -5.4; FTIR $\left(\mathrm{CH}_{2} \mathrm{Cl}_{2}\right)$ 2953, 2930, 2857, 1732, 1696, 1616, 1471, 1440, 1365, 1330, 1255, 1134, 840, $777 \mathrm{~cm}^{-1}$; HRMS (ESI-TOF) Calcd for $\mathrm{C}_{26} \mathrm{H}_{36} \mathrm{~N}_{2} \mathrm{NaO}_{5} \mathrm{Si}[\mathrm{M}+\mathrm{Na}]^{+}$507.2291, found 507.2290; $[\alpha]_{\mathrm{D}}^{25}=+140.5$ (c 1.0, $\mathrm{CHCl}_{3}$ ).

\section{Methyl $(R, Z)-13 a-(h y d r o x y m e t h y l)-8-m e t h o x y-3-o x o-1,2,3,5,6,13 a-h e x a h y d r o-11 \mathrm{H}-p y r r o l o[1 ', 2 ': 1,8] a z o c i n o[5,4-$ b]indole-11-carboxylate (29)}
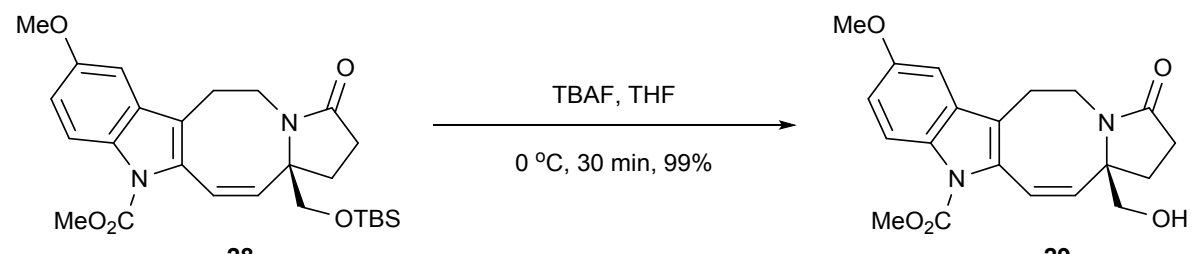

28

Scheme S18. Desilylation of 28.

To a solution of compound $28(30 \mathrm{mg}, 0.06 \mathrm{mmol})$ in THF $(0.6 \mathrm{~mL})$ was added TBAF $(0.07 \mathrm{~mL}, 1 \mathrm{M}$ in THF, 1.2 equiv.) at $0{ }^{\circ} \mathrm{C}$. After 30 min at $0{ }^{\circ} \mathrm{C}$, the reaction mixture was directly purified by silica-gel column chromatography (100\% EtOAc) to give the alcohol 29 (24 mg, $0.06 \mathrm{~mol})$ in $99 \%$ yield.

$\mathrm{R}_{\mathrm{f}}=0.2(100 \% \mathrm{EtOAc}) ;{ }^{1} \mathrm{H}$ NMR $\left(400 \mathrm{MHz}, \mathrm{CDCl}_{3}\right) \delta 7.88(\mathrm{~d}, J=9.0 \mathrm{~Hz}, 1 \mathrm{H}), 6.96(\mathrm{~d}, J=2.5 \mathrm{~Hz}, 1 \mathrm{H}), 6.87(\mathrm{dd}, J=9.0$, $2.5 \mathrm{~Hz}, 1 \mathrm{H}), 6.64(\mathrm{~d}, J=12.4 \mathrm{~Hz}, 1 \mathrm{H}), 5.42(\mathrm{~d}, J=12.4 \mathrm{~Hz}, 1 \mathrm{H}), 4.05(\mathrm{td}, J=13.4,4.6 \mathrm{~Hz}, 1 \mathrm{H}), 4.00(\mathrm{~s}, 3 \mathrm{H}), 3.92(\mathrm{~d}, J=$ $11.7 \mathrm{~Hz}, 1 \mathrm{H}), 3.86(\mathrm{~s}, 3 \mathrm{H}), 3.66(\mathrm{~d}, J=11.6 \mathrm{~Hz}, 1 \mathrm{H}), 3.07(\mathrm{dd}, J=13.4,5.2 \mathrm{~Hz}, 1 \mathrm{H}), 2.96(\mathrm{ddd}, J=14.0,4.5,1.2 \mathrm{~Hz}, 1 \mathrm{H})$, $2.69(\mathrm{td}, J=13.7,5.6 \mathrm{~Hz}, 1 \mathrm{H}), 2.40(\mathrm{br}, 1 \mathrm{H}), 2.35-2.25(\mathrm{~m}, 1 \mathrm{H}), 2.18-2.00(\mathrm{~m}, 2 \mathrm{H}), 1.91-1.85(\mathrm{~m}, 1 \mathrm{H}) ;{ }^{13} \mathrm{C} \mathrm{NMR}(100 \mathrm{MHz}$, $\left.\mathrm{CDCl}_{3}\right) \delta$ 176.0, 156.4, 152.5, 134.3, 130.6, 130.0, 128.4, 121.3, 117.7, 116.6, 113.5, 101.1, 68.2, 66.1, 55.8, 53.6, 37.0, 30.5, 28.9, 22.4; FT-IR $\left(\mathrm{CH}_{2} \mathrm{Cl}_{2}\right) 3377,2954,2932,1730,1667,1441,1367,1328,1257,1134,1083,1145,806,765 \mathrm{~cm}^{-1}$; HRMS (ESI-TOF) Calcd for $\mathrm{C}_{20} \mathrm{H}_{22} \mathrm{~N}_{2} \mathrm{NaO}_{5}[\mathrm{M}+\mathrm{Na}]^{+} 393.1426$, found 393.1429; $[\alpha]_{\mathrm{D}}^{25}=+135.9$ (c 1.0, $\mathrm{CHCl}_{3}$ ); HPLC 
(Chiralpak OD, hexane/i-PrOH $=80: 20$, flow rate $1 \mathrm{~mL} / \mathrm{min}$ ), $t_{\mathrm{R}}=19.320$ (minor), 10.541 (major) $\mathrm{min}$; ee $=87 \%$. 


\section{NMR Spectra}

${ }^{1} \mathrm{H}$ NMR of Compound $10 \mathrm{e}\left(400 \mathrm{MHz}, \mathrm{CDCl}_{3}\right)$

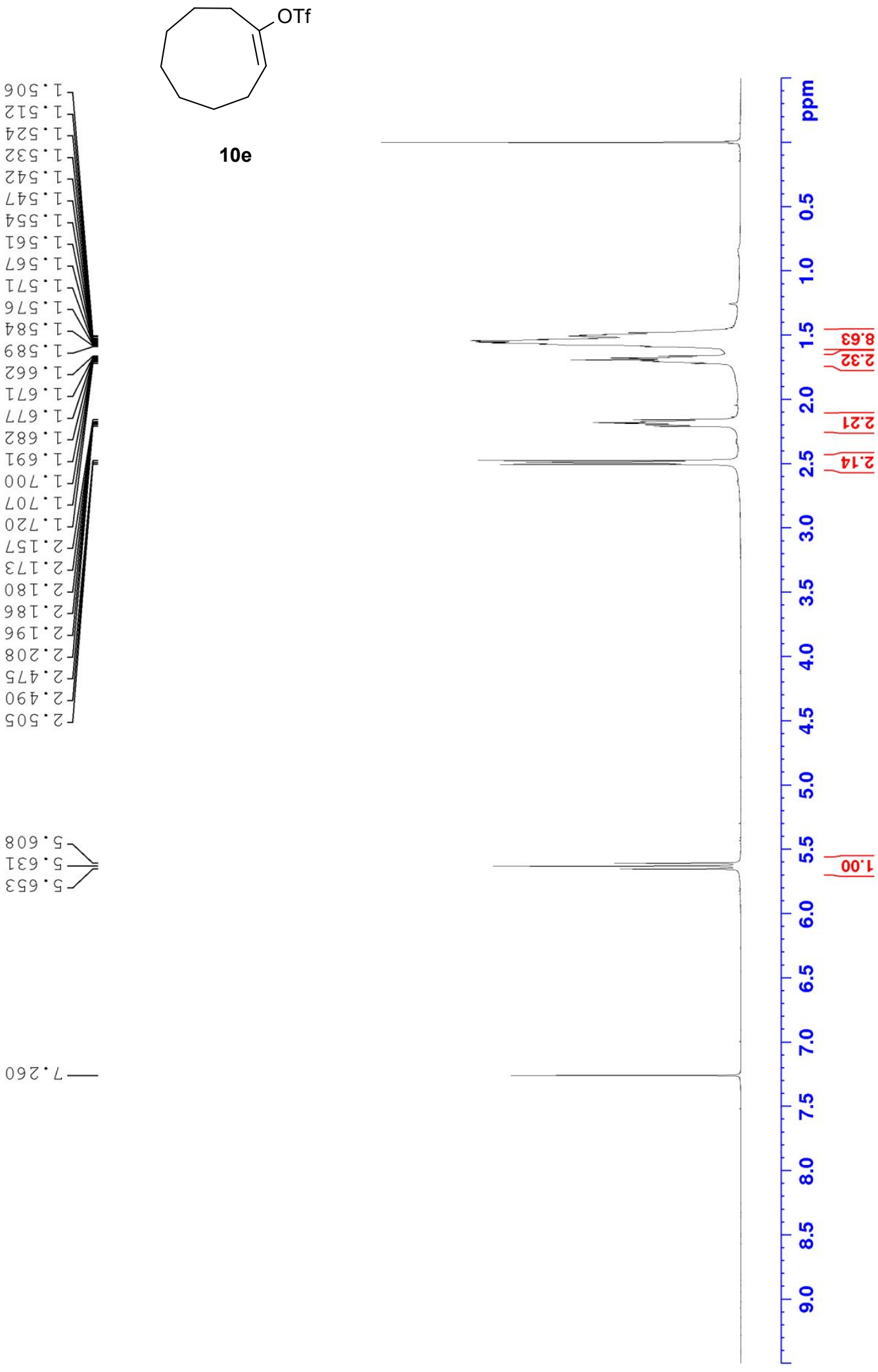


${ }^{13} \mathrm{C}$ NMR of Compound $10 \mathrm{e}\left(100 \mathrm{MHz}, \mathrm{CDCl}_{3}\right)$

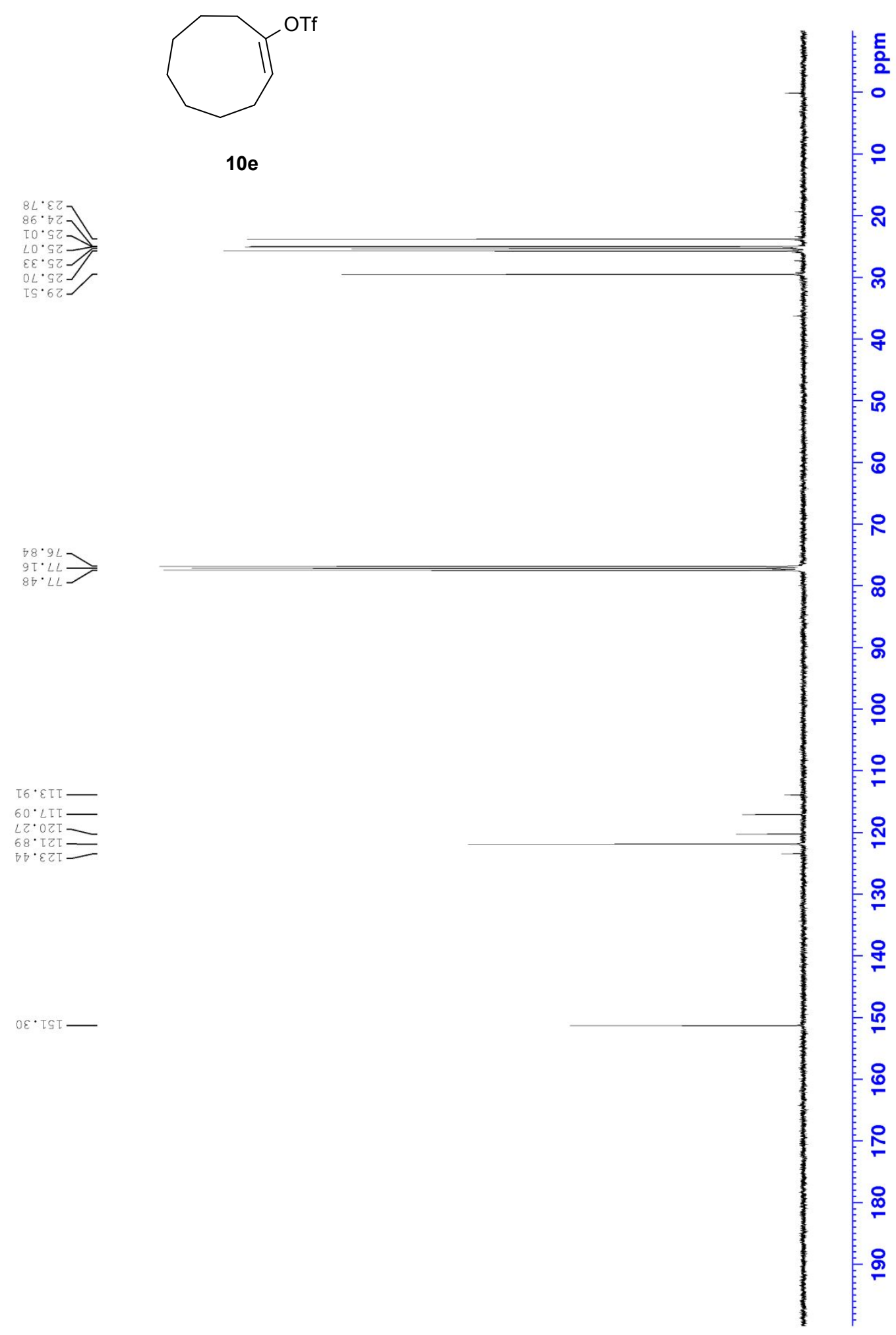


${ }^{1} \mathrm{H}$ NMR of Compound $11 \mathrm{~b}\left(400 \mathrm{MHz}, \mathrm{CDCl}_{3}\right)$

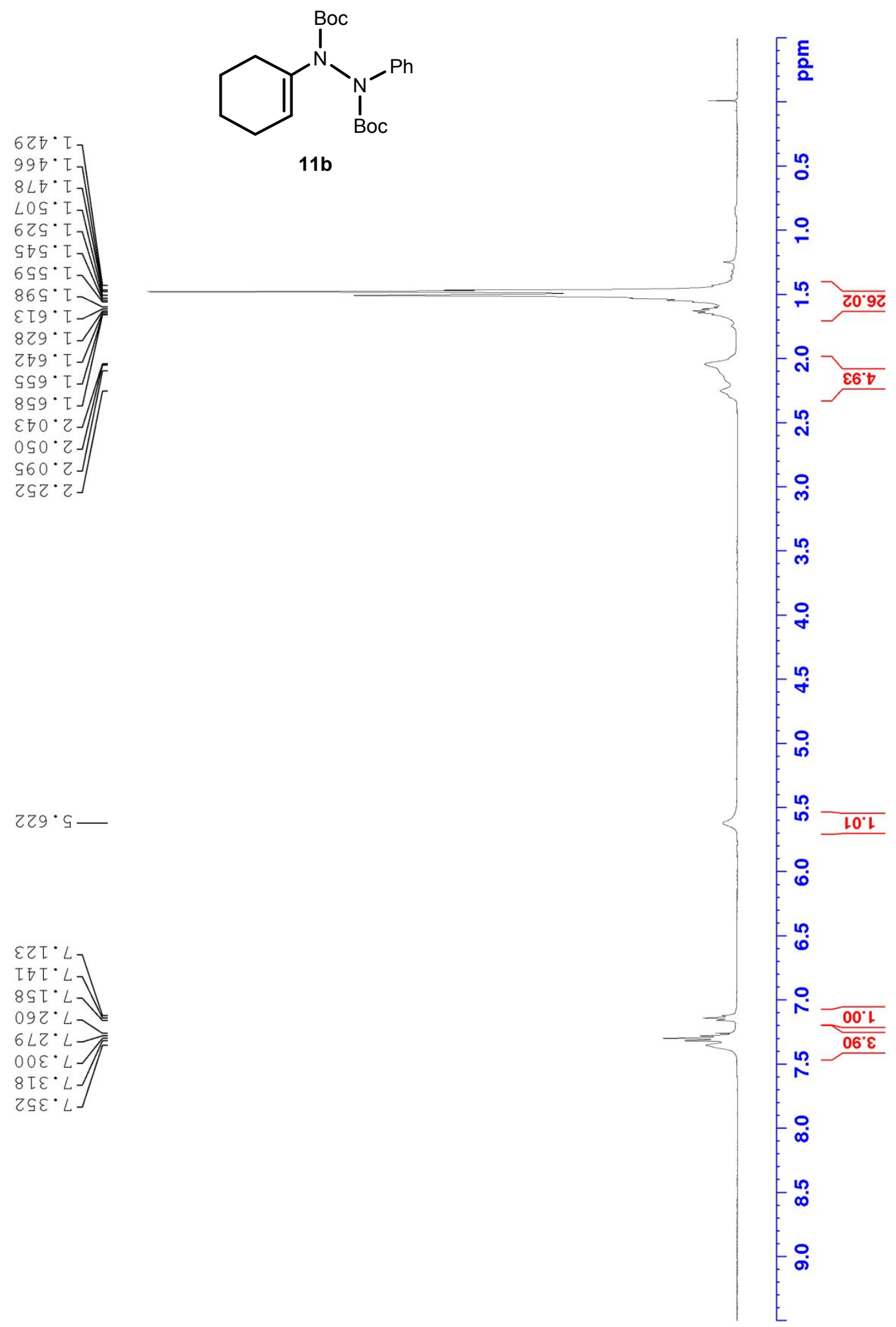


${ }^{13} \mathrm{C}$ NMR of Compound 11b (100 MHz, $\left.\mathrm{CDCl}_{3}\right)$

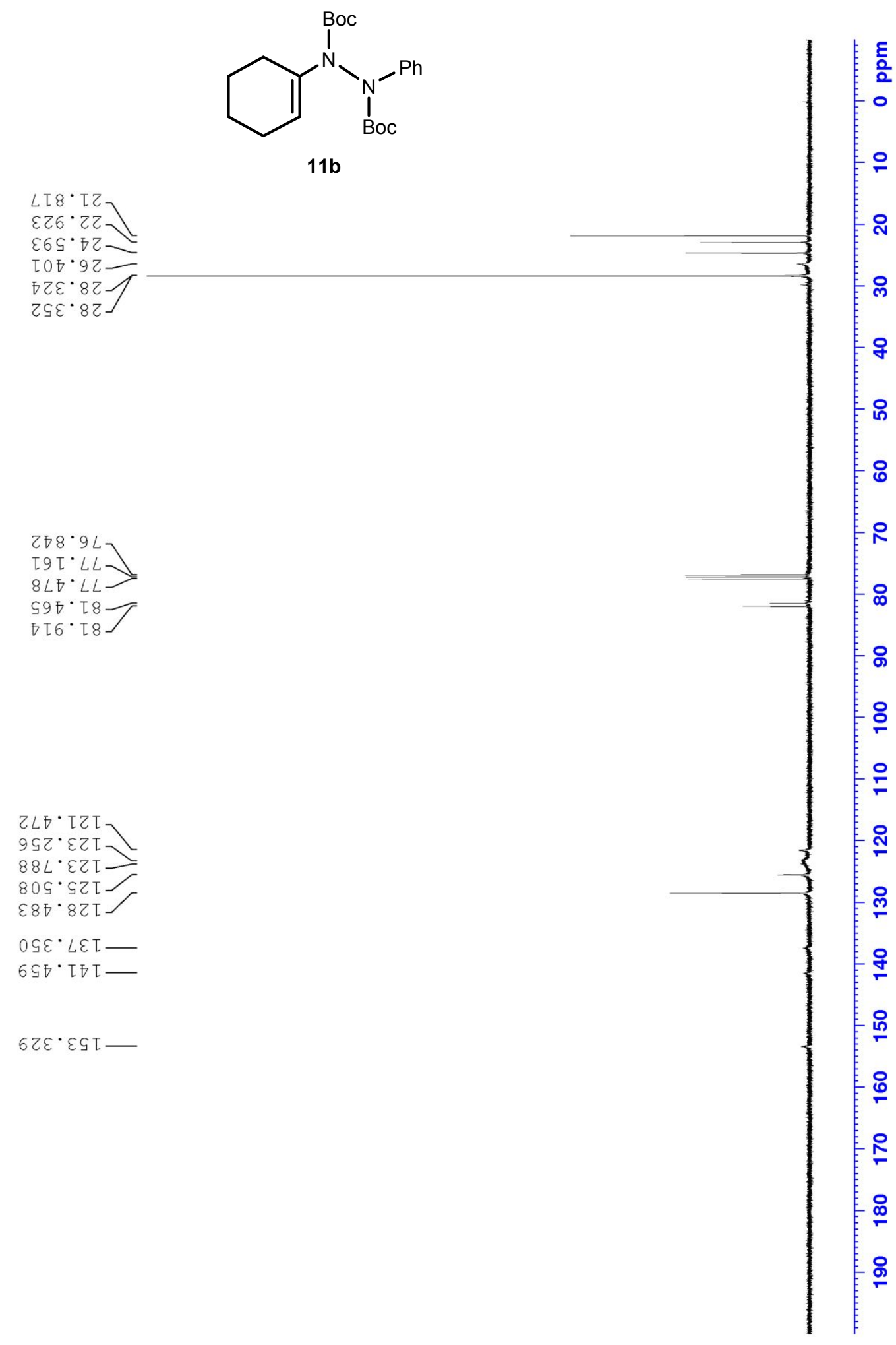


${ }^{1} \mathrm{H}$ NMR of Compound 11c (400 MHz, $\left.\mathrm{CDCl}_{3}\right)$
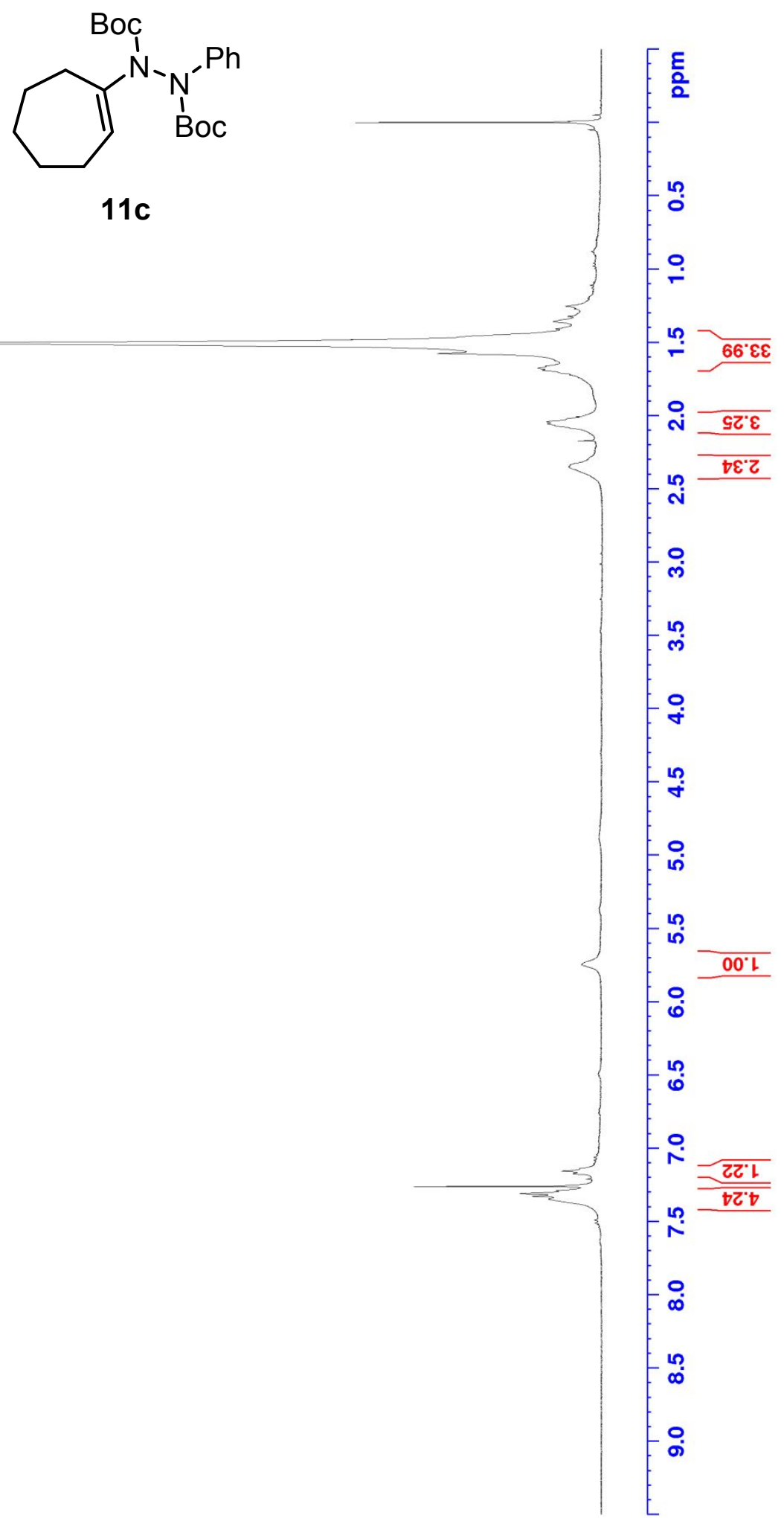
${ }^{13} \mathrm{C}$ NMR of Compound 11c (100 MHz, $\left.\mathrm{CDCl}_{3}\right)$

$20 L \cdot 92$

$968 \cdot 92$

$08 \varepsilon \cdot 82$

$8 L 0^{82}$

५60. $2 \varepsilon$

$0 \varepsilon \tau^{*} \cdot \tau \varepsilon$

$\varepsilon \mp 8 \cdot 9 L$

$09[\cdot L L$

$8 L F^{\circ} \cdot L L$

$9 L G \cdot 18-T$

6 [6. ${ }^{2} 8$

$889 \cdot 92 t$

$9 S L \cdot S Z T$

ZSG. $82 \mathrm{~T}$

$0 L 6 \cdot 6 Z$ T

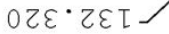

ธ०००० 0 โ

ESS'ItI

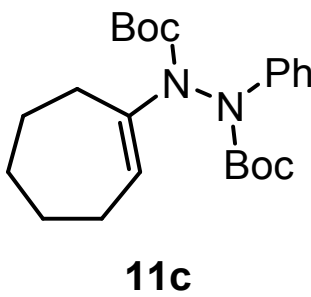

$11 \mathrm{c}$

$O L I \cdot L G T$ 
${ }^{1} \mathrm{H}$ NMR of Compound 11d (400 MHz, DMSO- $\mathrm{d}_{6}$ )

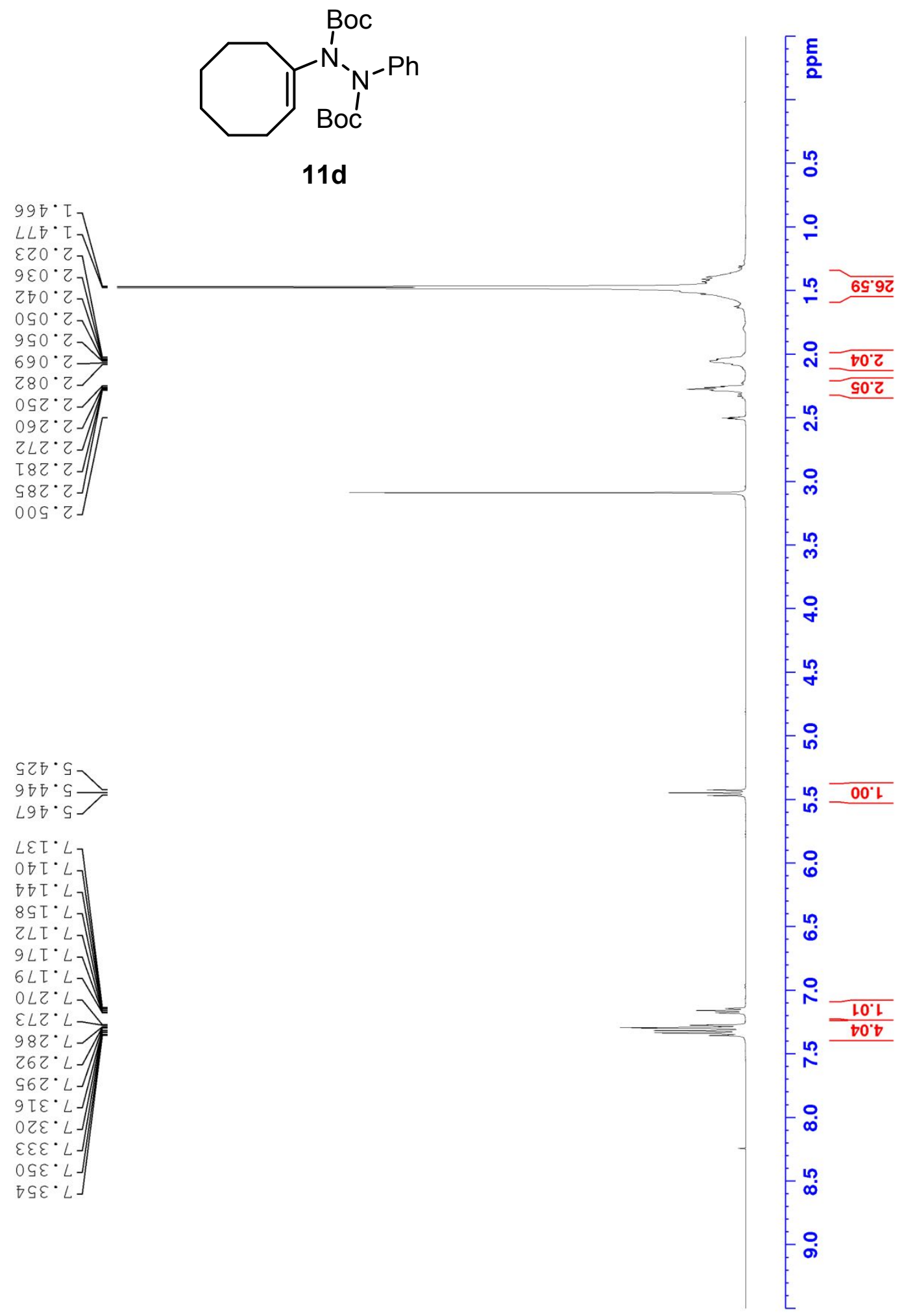


${ }^{13} \mathrm{C}$ NMR of Compound 11d (100 MHz, DMSO- $\mathrm{d}_{6}$ )

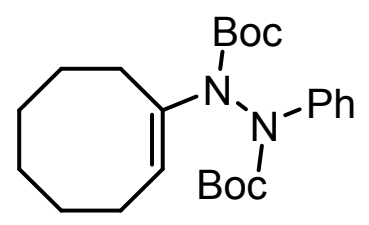

11d

11d

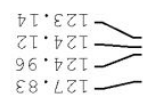

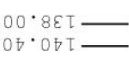

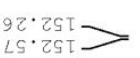

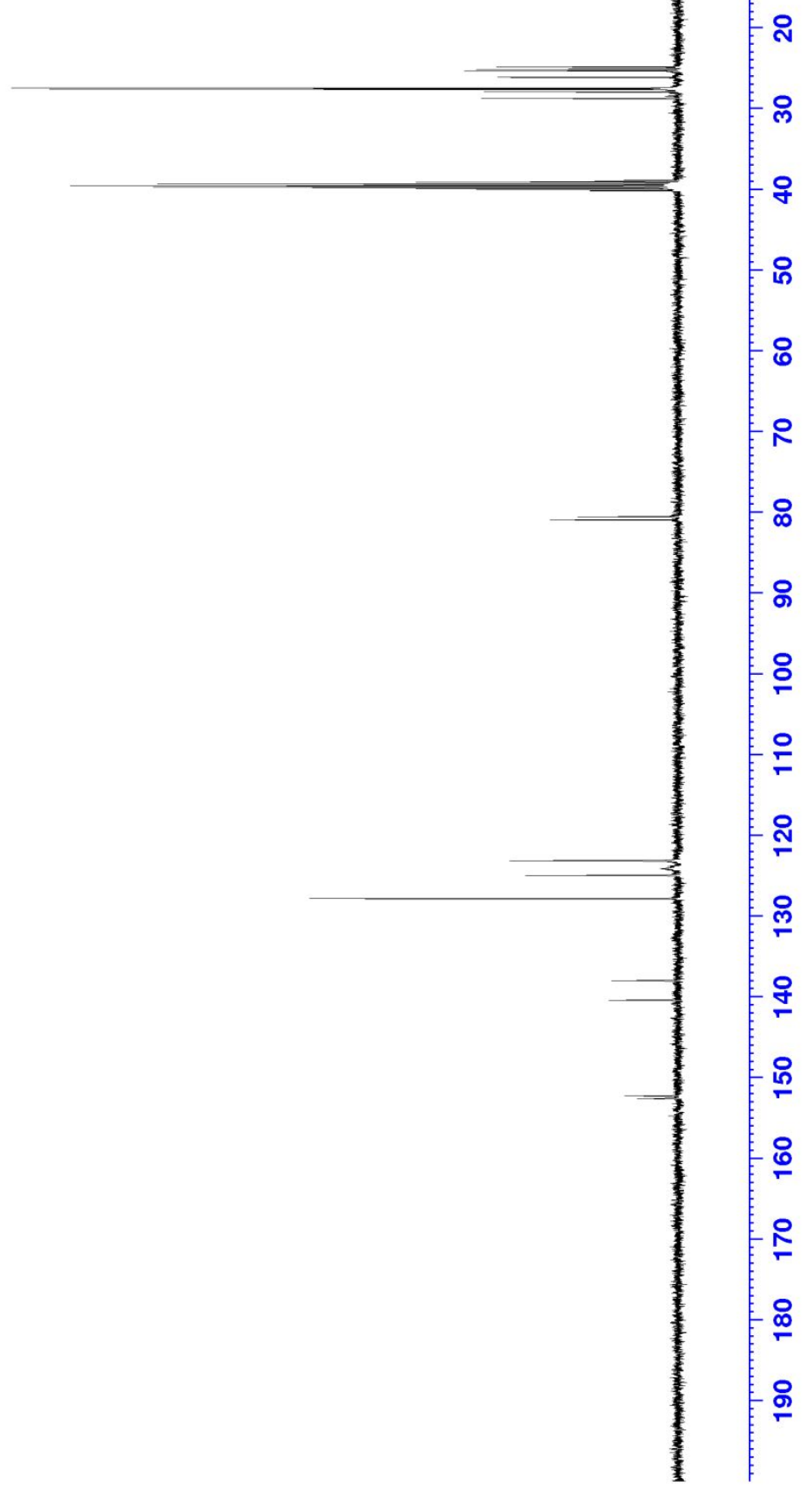


${ }^{1} \mathrm{H}$ NMR of Compound 11e (400 MHz, DMSO-d $\left.\mathrm{d}_{6}\right)$

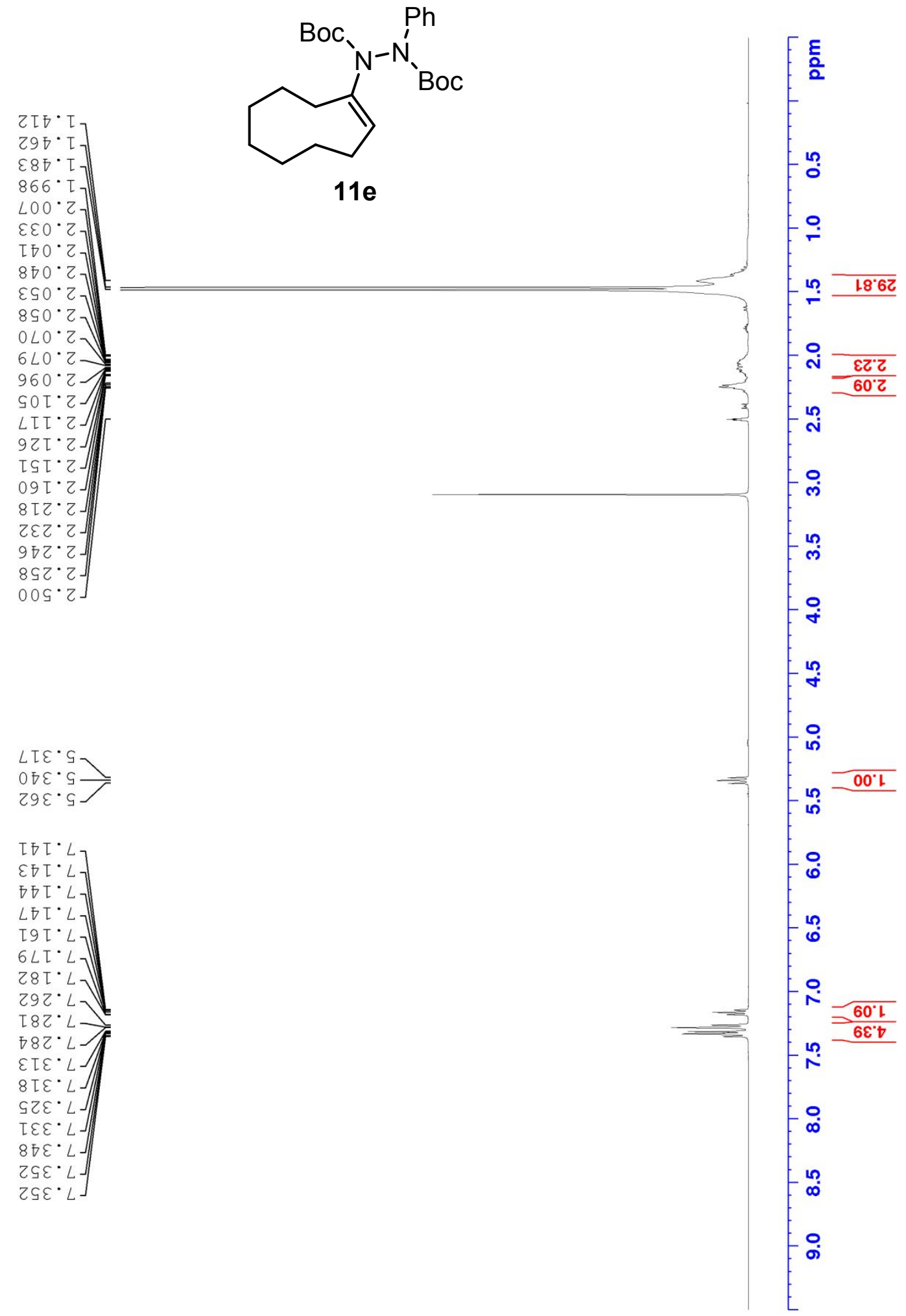


${ }^{13} \mathrm{C}$ NMR of Compound 11e (100 MHz, DMSO- $\left.\mathrm{d}_{6}\right)$
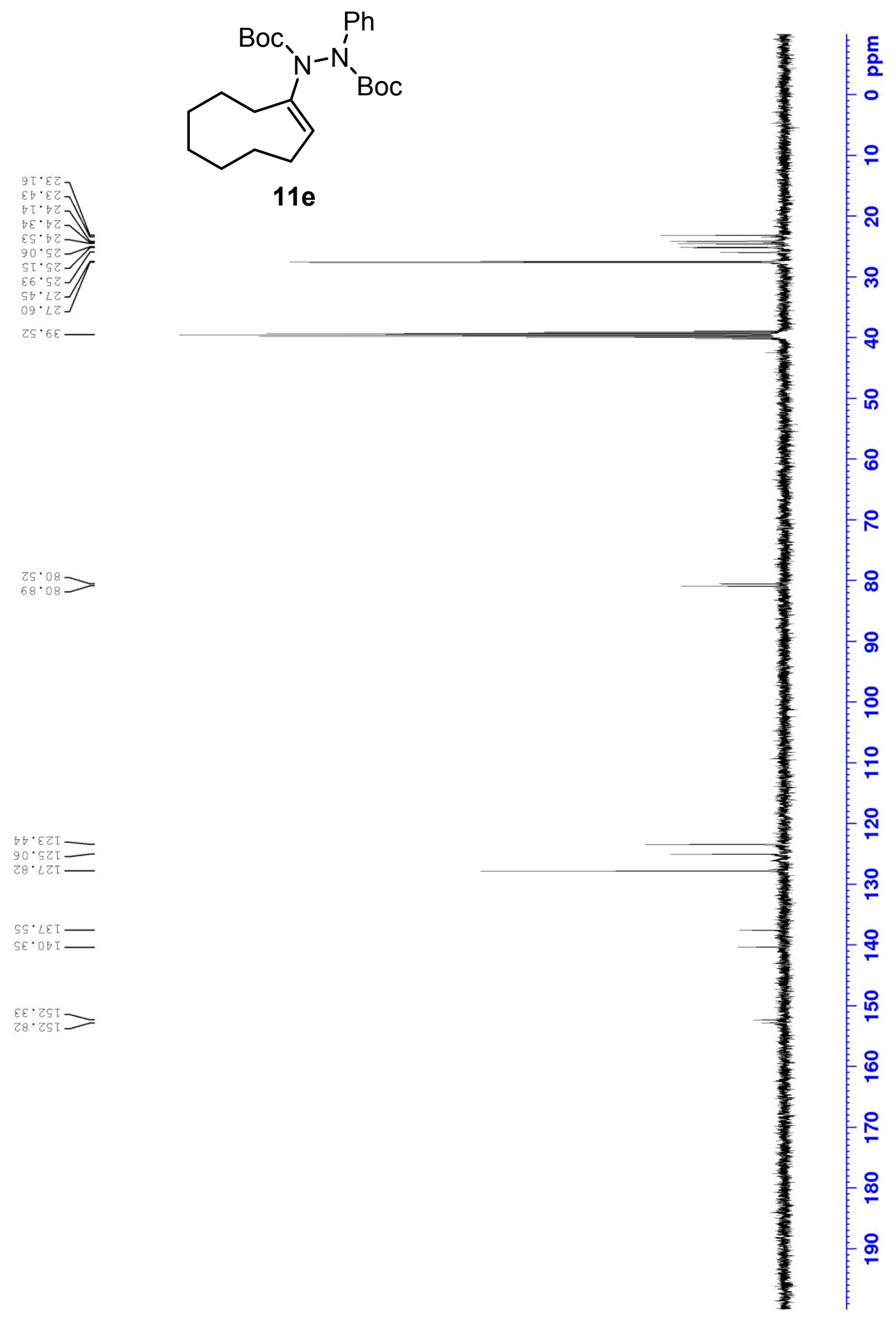
${ }^{1} \mathrm{H}$ NMR of Compound pre-11e' $\left(400 \mathrm{MHz}, \mathrm{DMSO}-\mathrm{d}_{6}\right)$

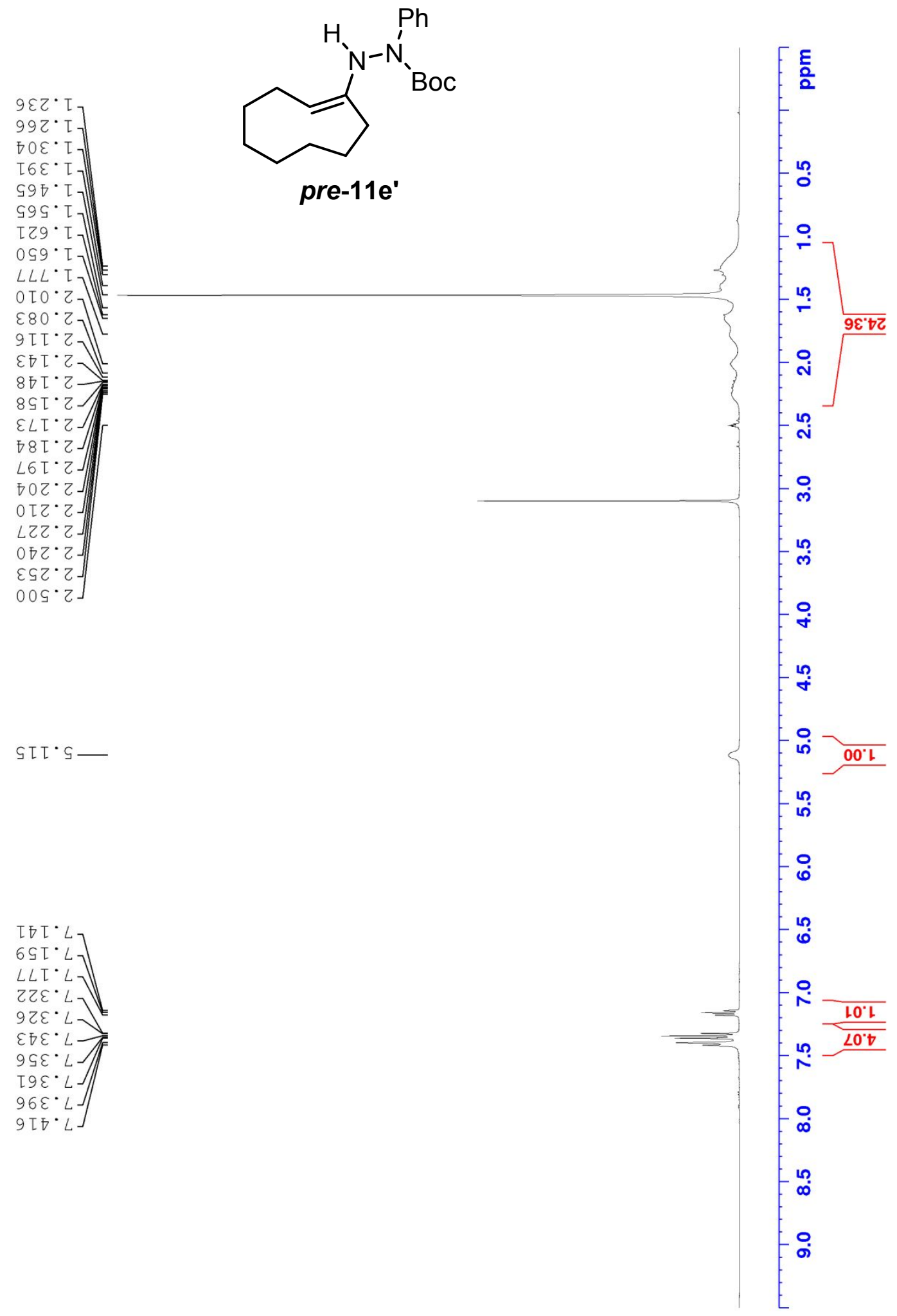


${ }^{13} \mathrm{C}$ NMR of Compound pre-11e' (100 MHz, DMSO-d $\left.\mathrm{d}_{6}\right)$

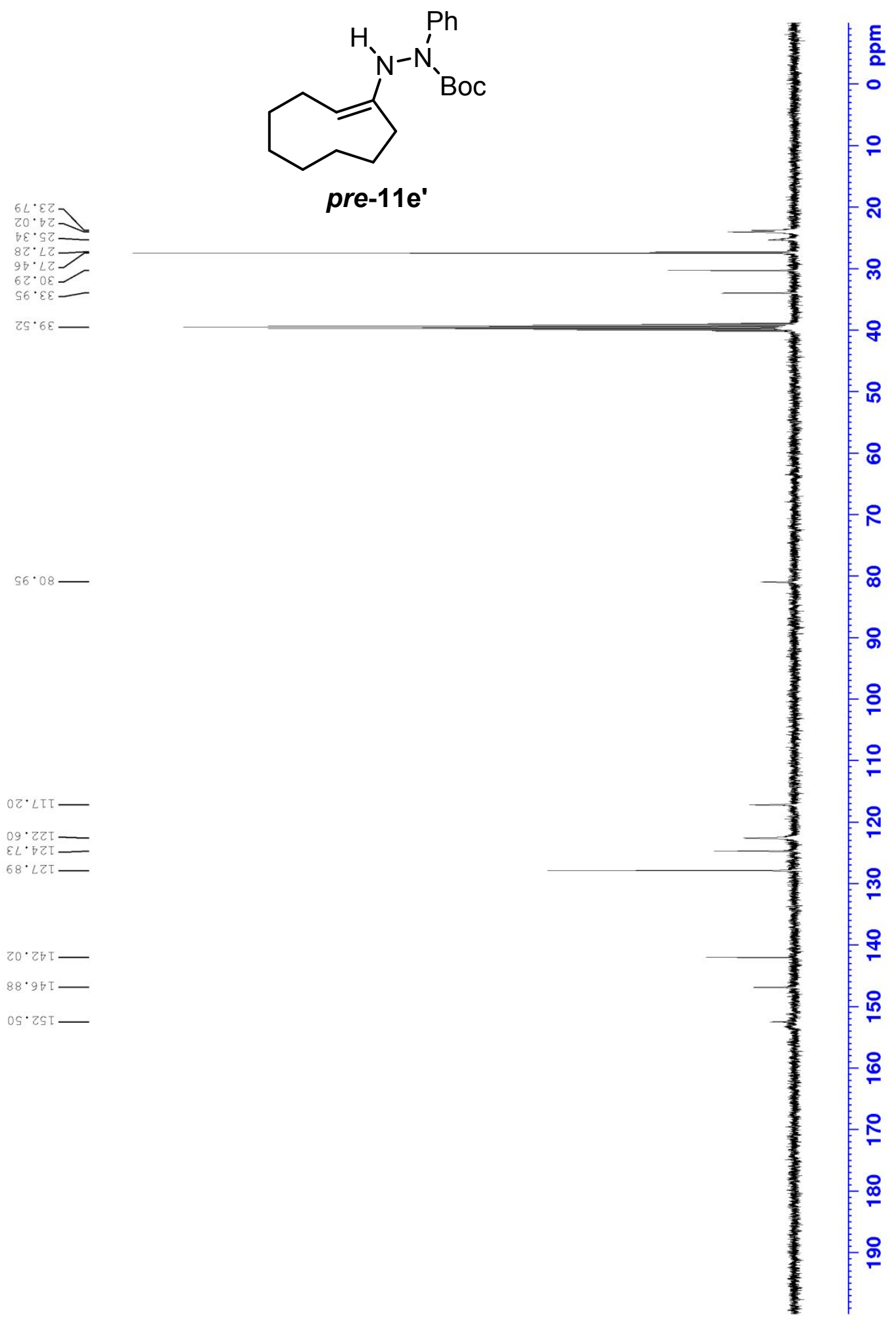


${ }^{1} \mathrm{H}$ NMR of Compound 11e' (400 MHz, $\left.\mathrm{CDCl}_{3}\right)$

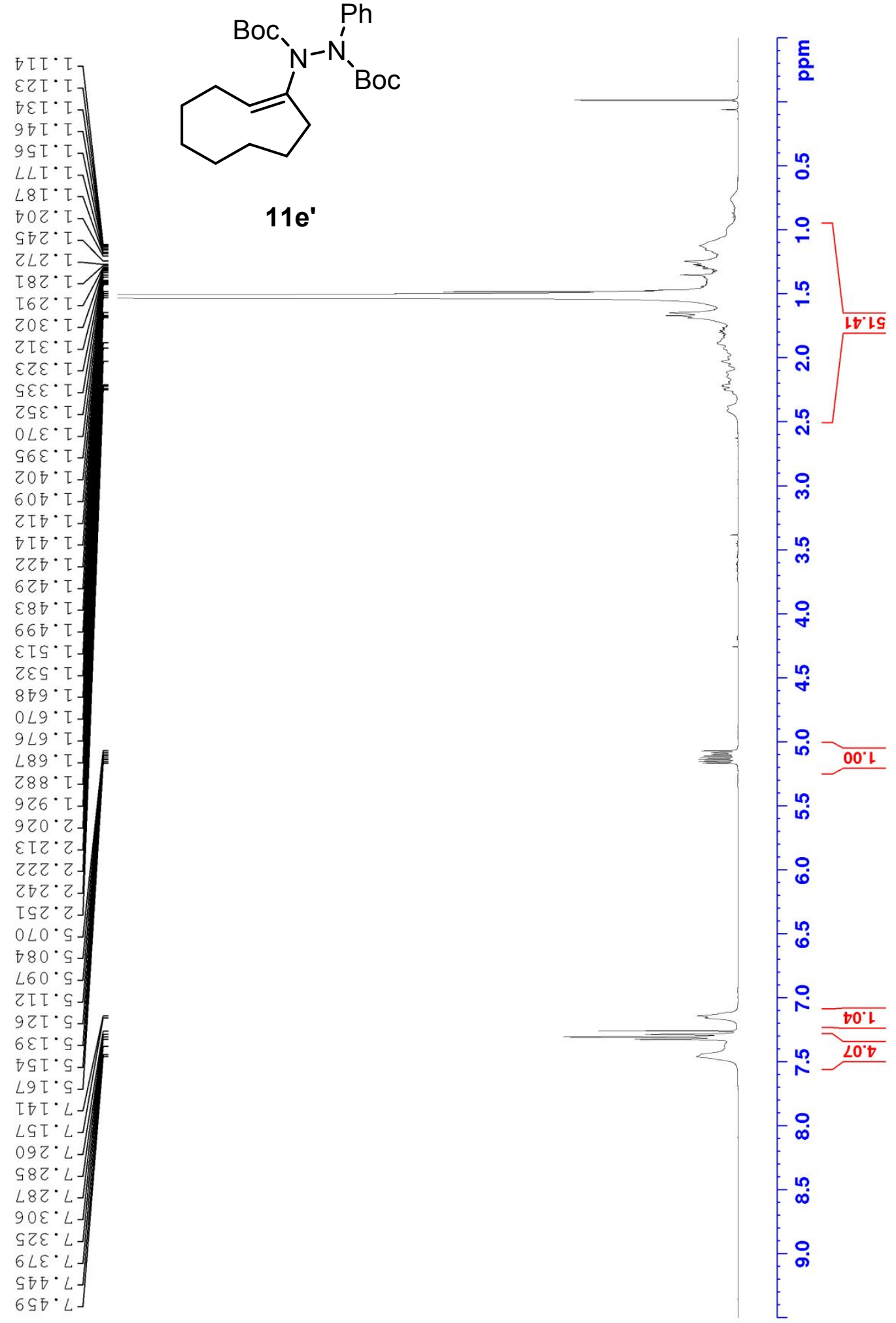


${ }^{13} \mathrm{C}$ NMR of Compound 11e' (100 MHz, DMSO-d $\left.d_{6}\right)$

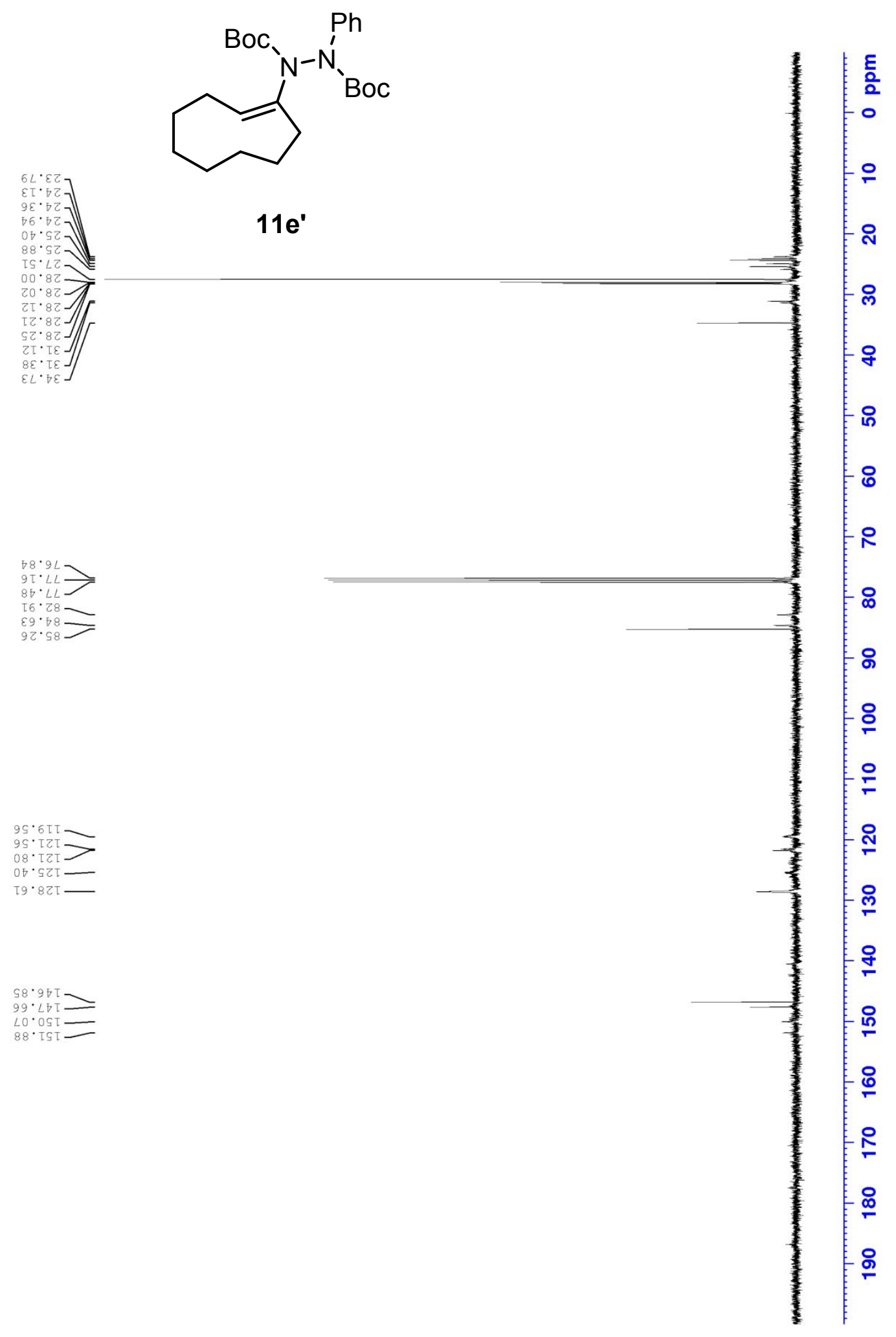


${ }^{1} \mathrm{H}$ NMR of $12 \mathrm{e}\left(400 \mathrm{MHz}, \mathrm{CDCl}_{3}\right)$

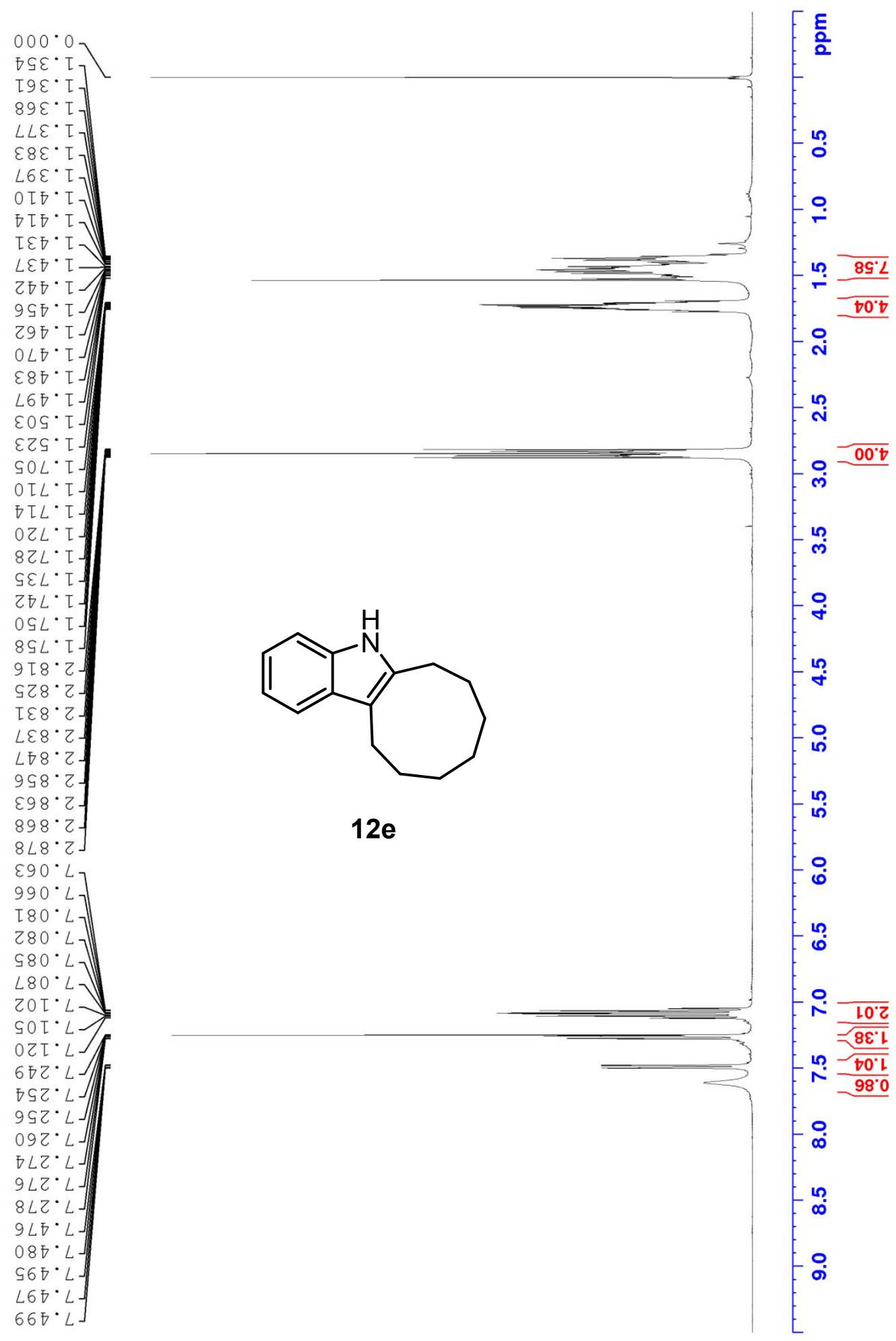


${ }^{13} \mathrm{C}$ NMR of $12 \mathrm{e}\left(100 \mathrm{MHz}, \mathrm{CDCl}_{3}\right)$

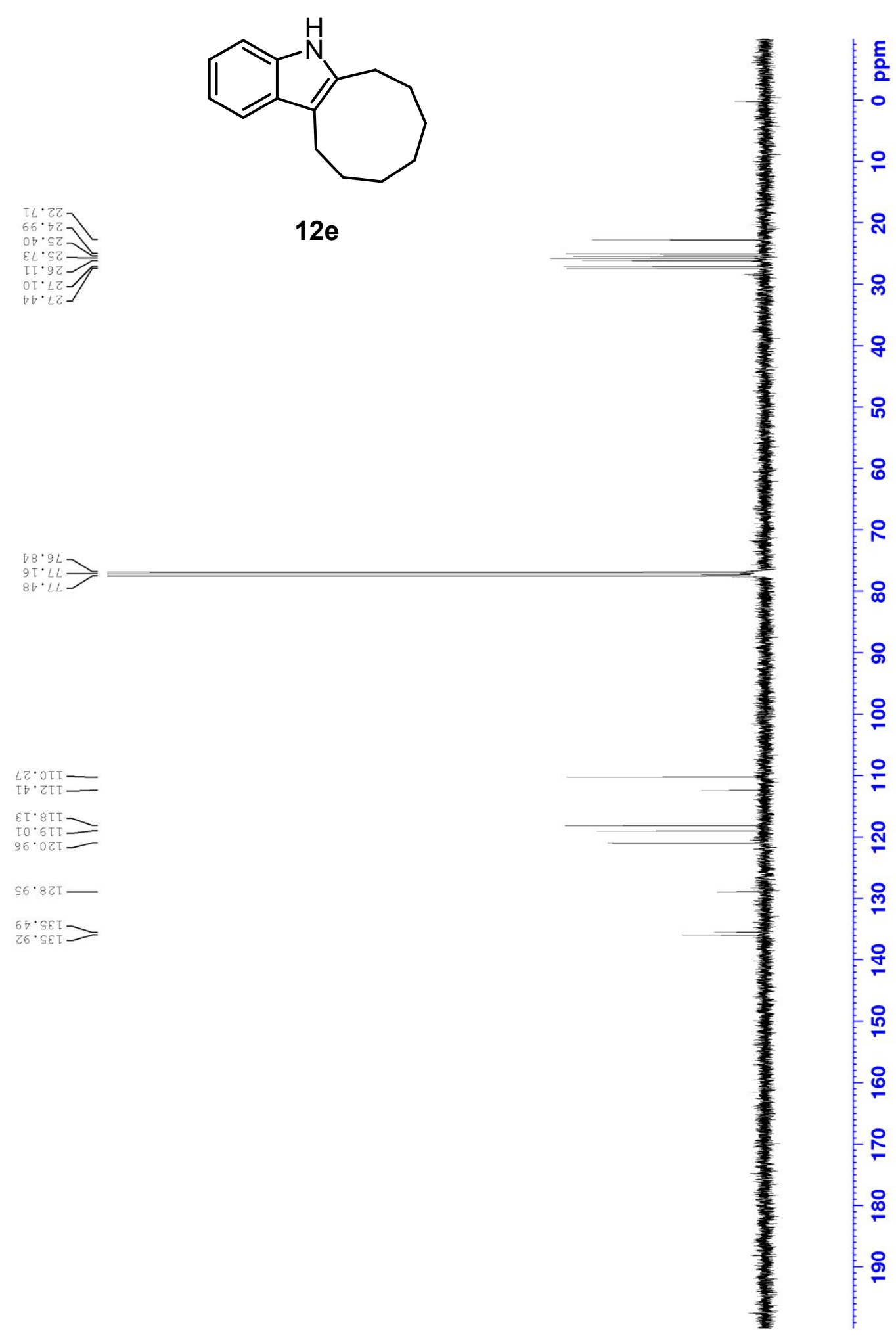


${ }^{1} \mathrm{H}$ NMR of $12 \mathrm{e}$ prepared from $11 \mathrm{e}$ and $11 \mathrm{e}$ ' $\left(400 \mathrm{MHz}, \mathrm{CDCl}_{3}\right)$

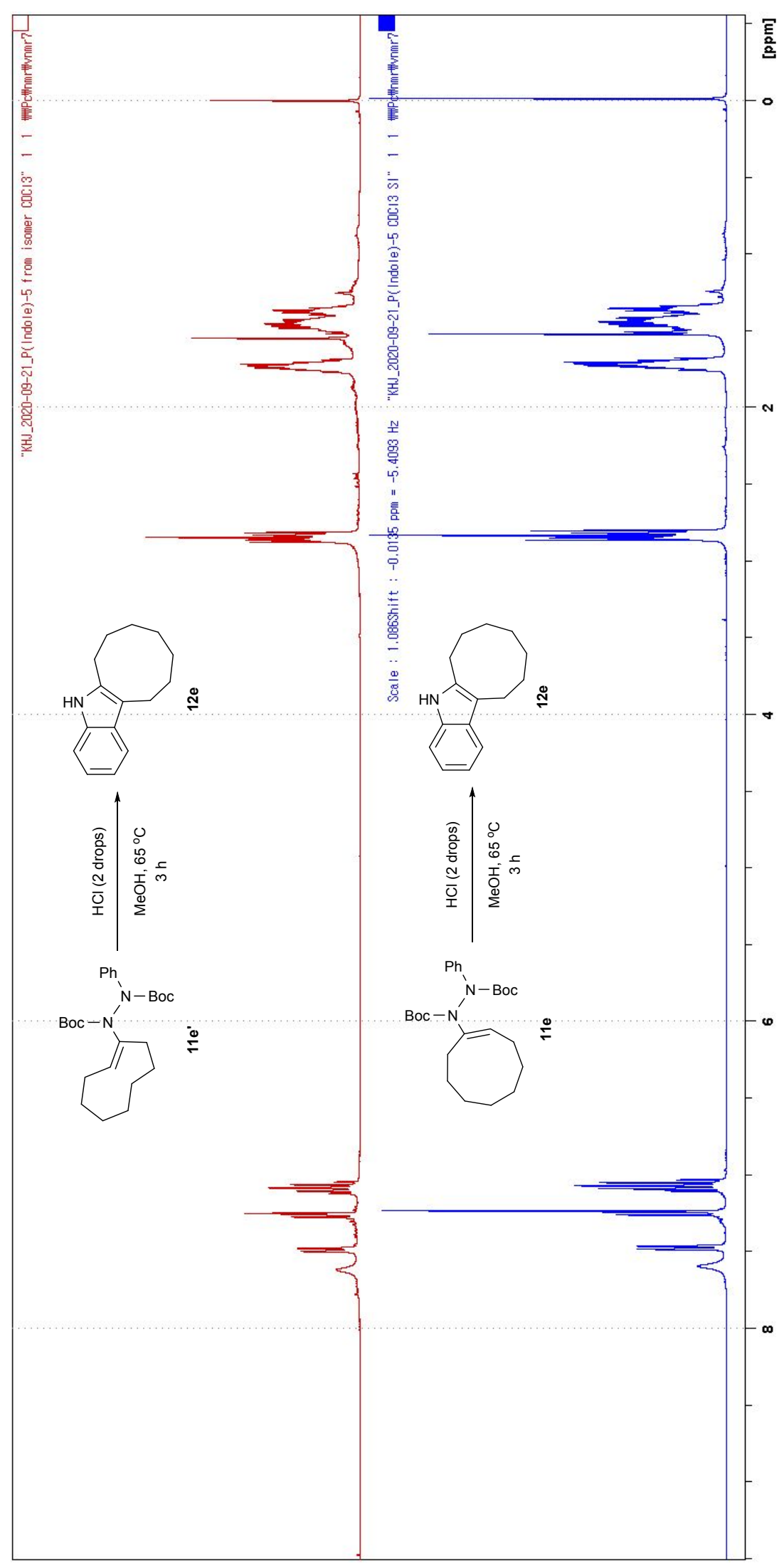


${ }^{13} \mathrm{C}$ NMR of $12 \mathrm{e}$ prepared from $11 \mathrm{e}$ and $11 \mathrm{e}$ ( $\left.100 \mathrm{MHz}, \mathrm{CDCl}_{3}\right)$

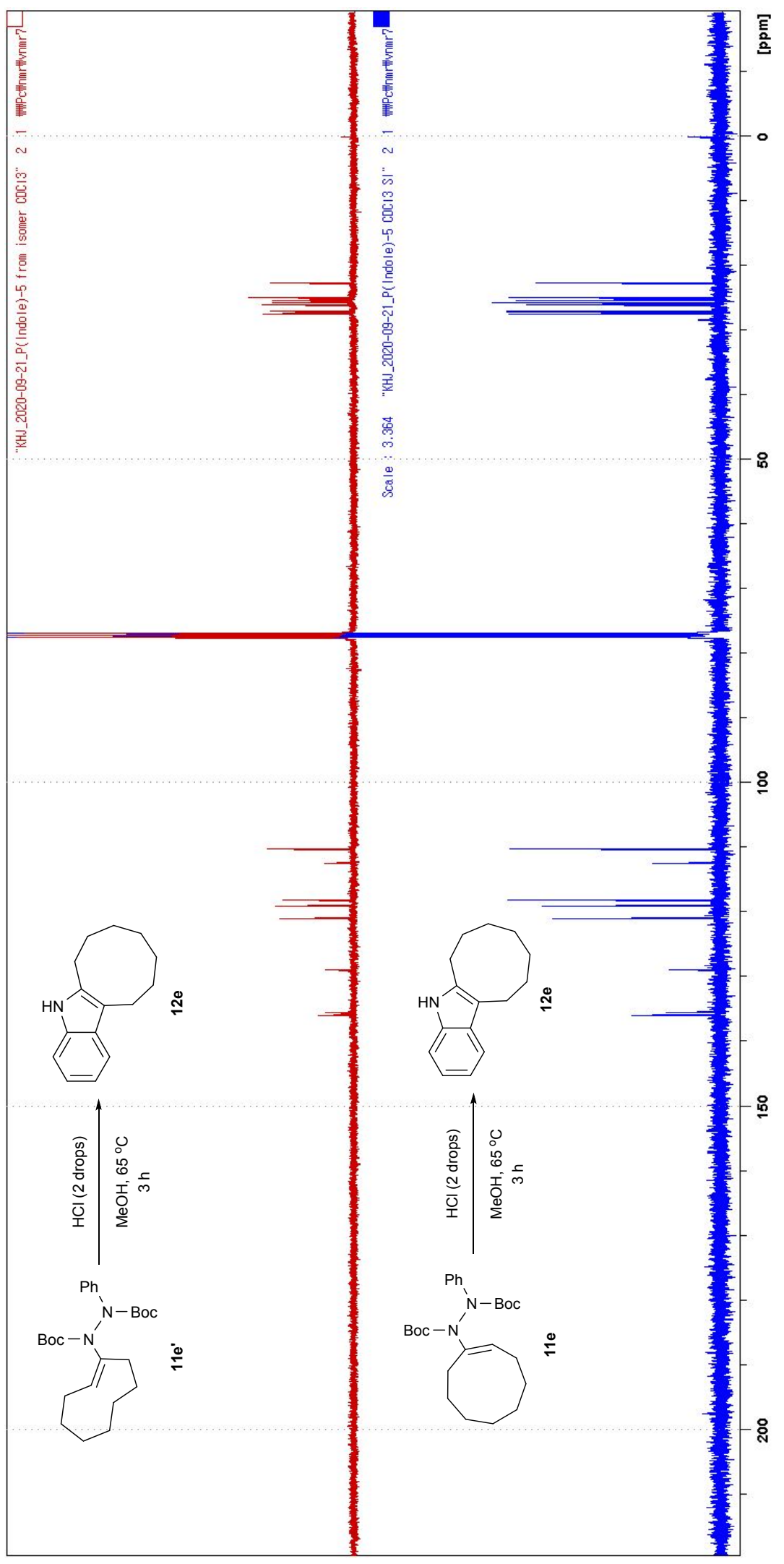


${ }^{1} \mathrm{H}-\mathrm{NMR}$ of Compound $14\left(400 \mathrm{MHz}, \mathrm{CDCl}_{3}\right)$

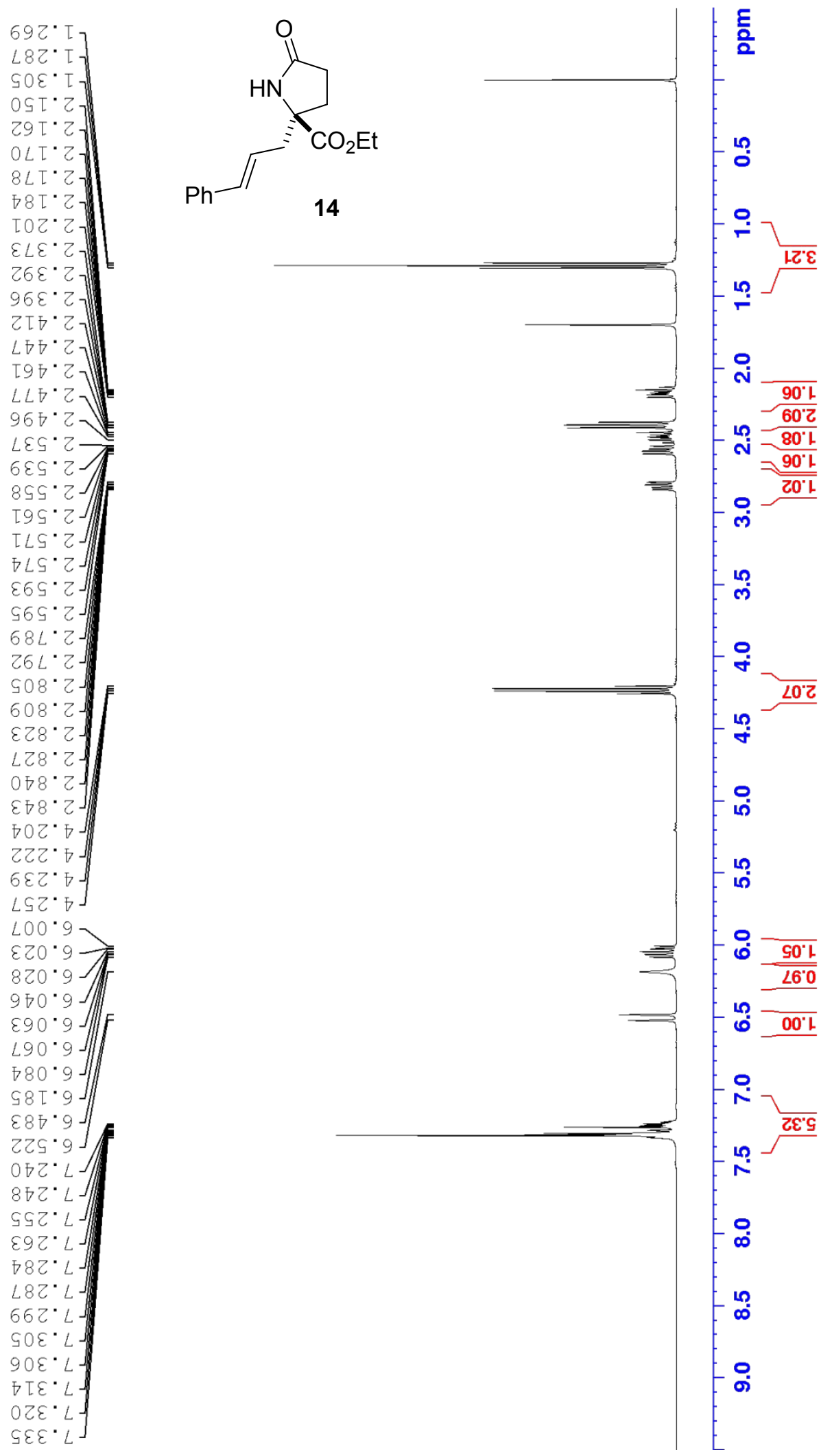


${ }^{13} \mathrm{C}$-NMR of Compound $14\left(100 \mathrm{MHz}, \mathrm{CDCl}_{3}\right)$

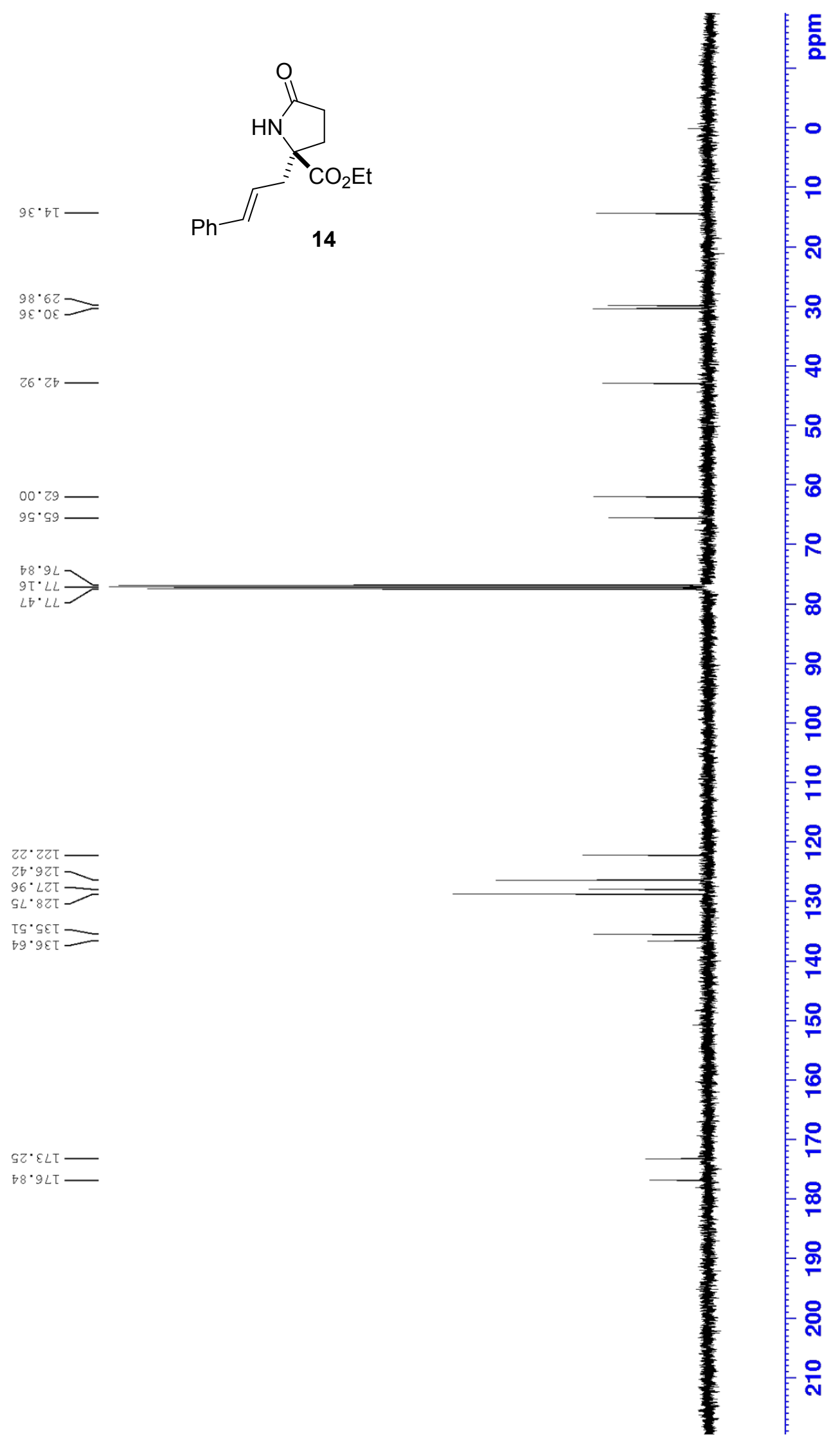


${ }^{1} \mathrm{H}-\mathrm{NMR}$ of Compound $16\left(400 \mathrm{MHz}, \mathrm{CDCl}_{3}\right)$

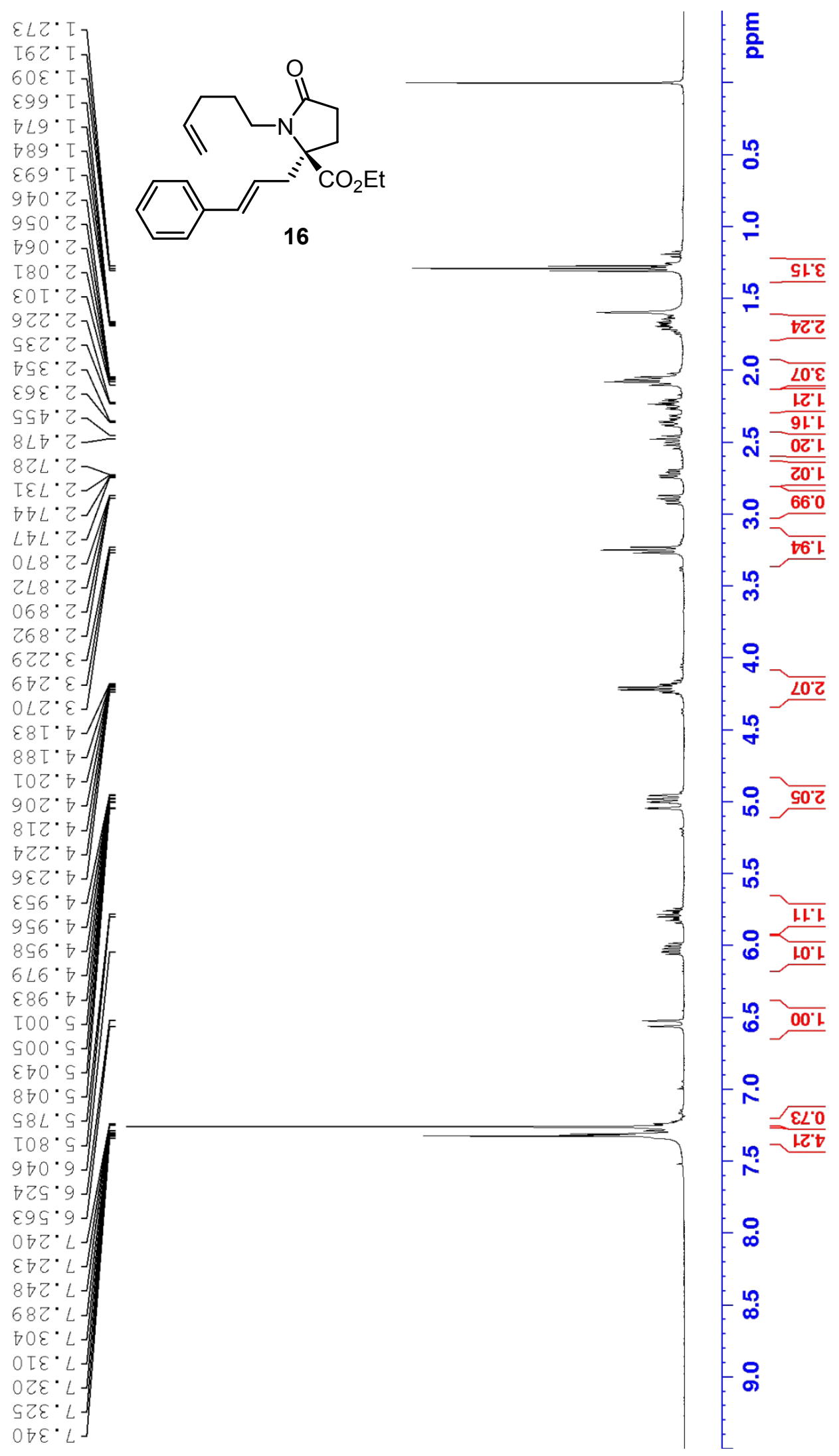


${ }^{13} \mathrm{C}$-NMR of Compound $16\left(100 \mathrm{MHz}, \mathrm{CDCl}_{3}\right)$

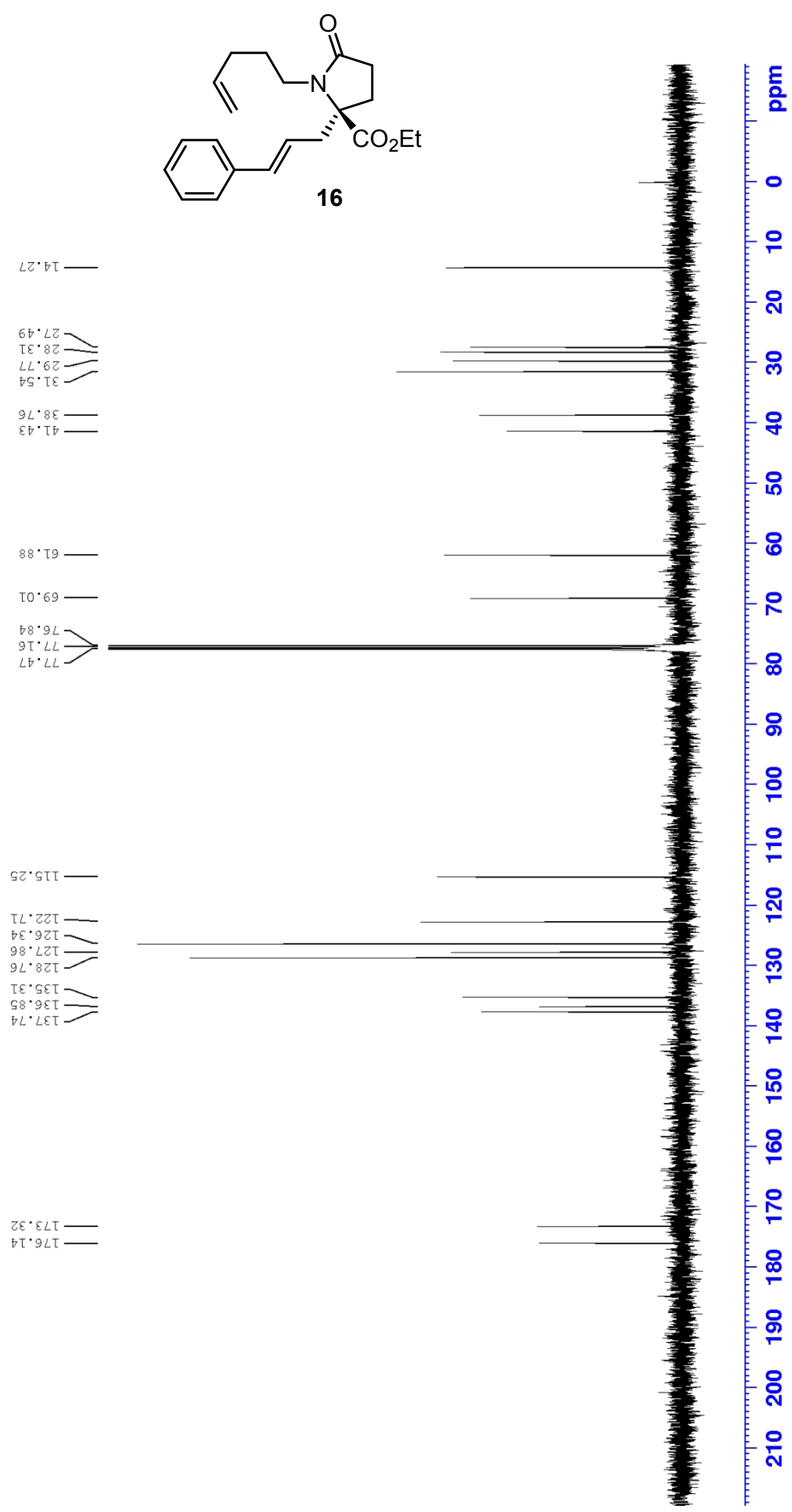


${ }^{1} \mathrm{H}-\mathrm{NMR}$ of Compound $17\left(400 \mathrm{MHz}, \mathrm{CDCl}_{3}\right)$

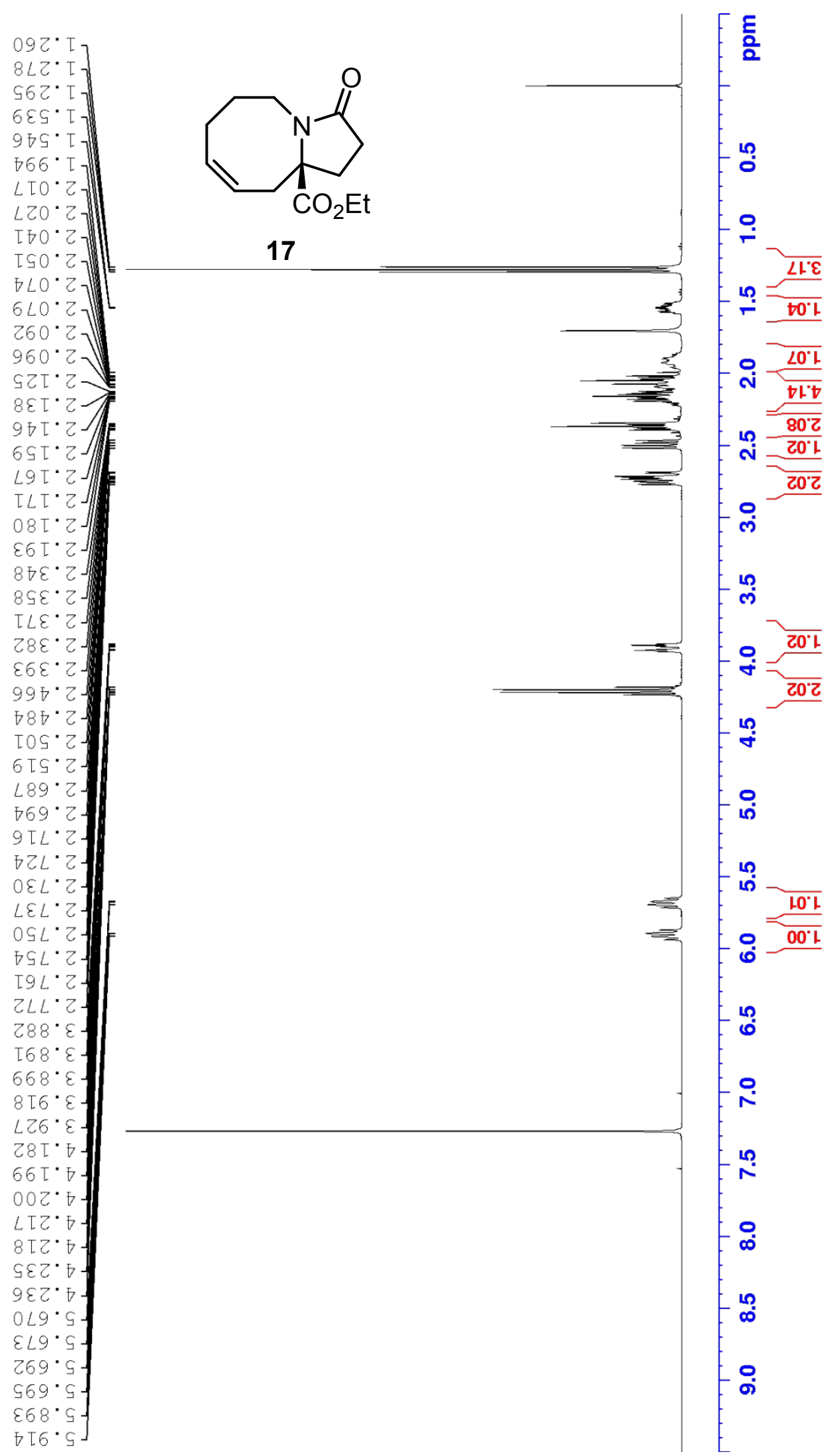

${ }^{13} \mathrm{C}$-NMR of Compound $17\left(100 \mathrm{MHz}, \mathrm{CDCl}_{3}\right)$ 


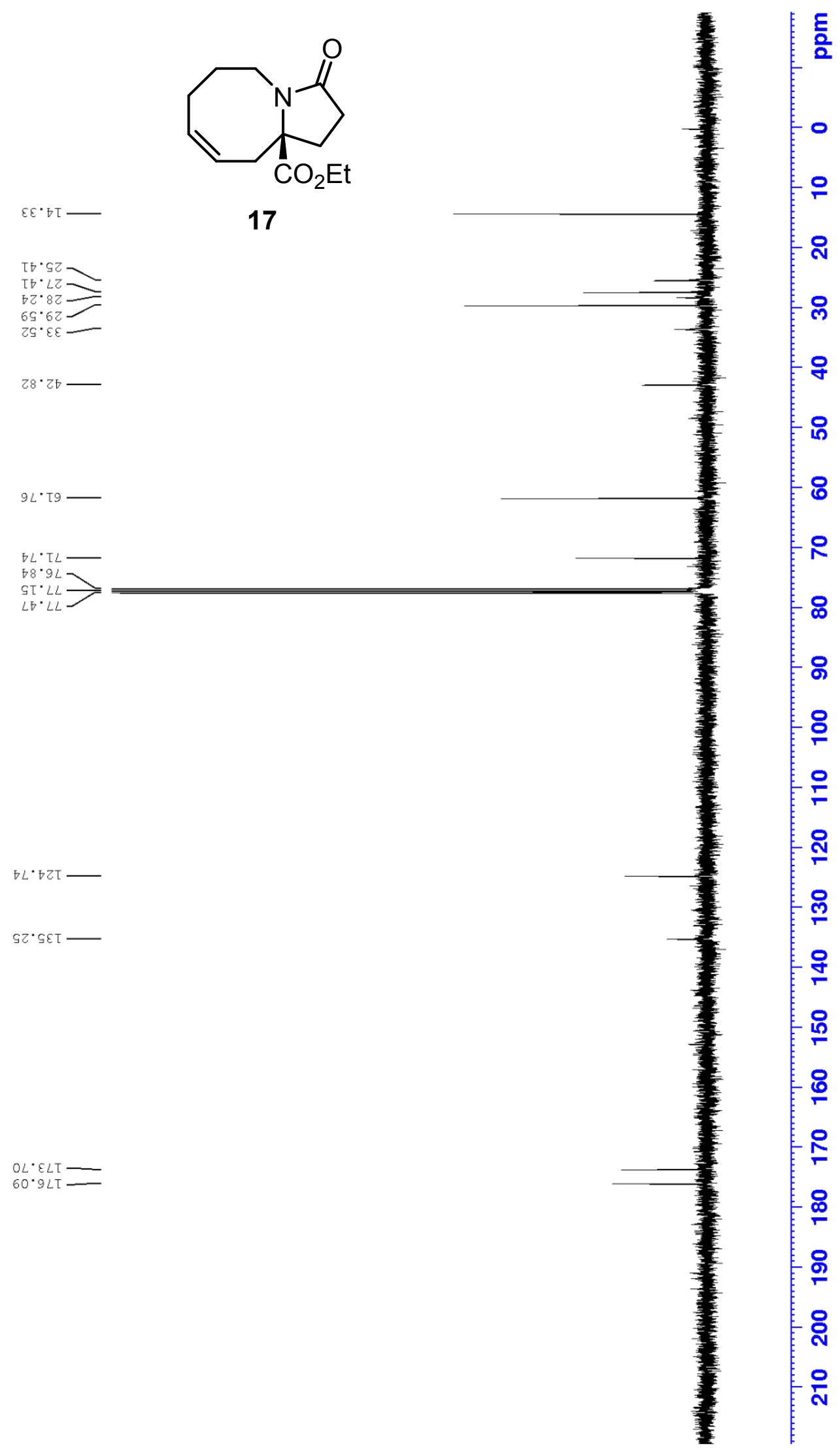

${ }^{1} \mathrm{H}-\mathrm{NMR}$ of Compound $18\left(400 \mathrm{MHz}, \mathrm{CDCl}_{3}\right)$ 


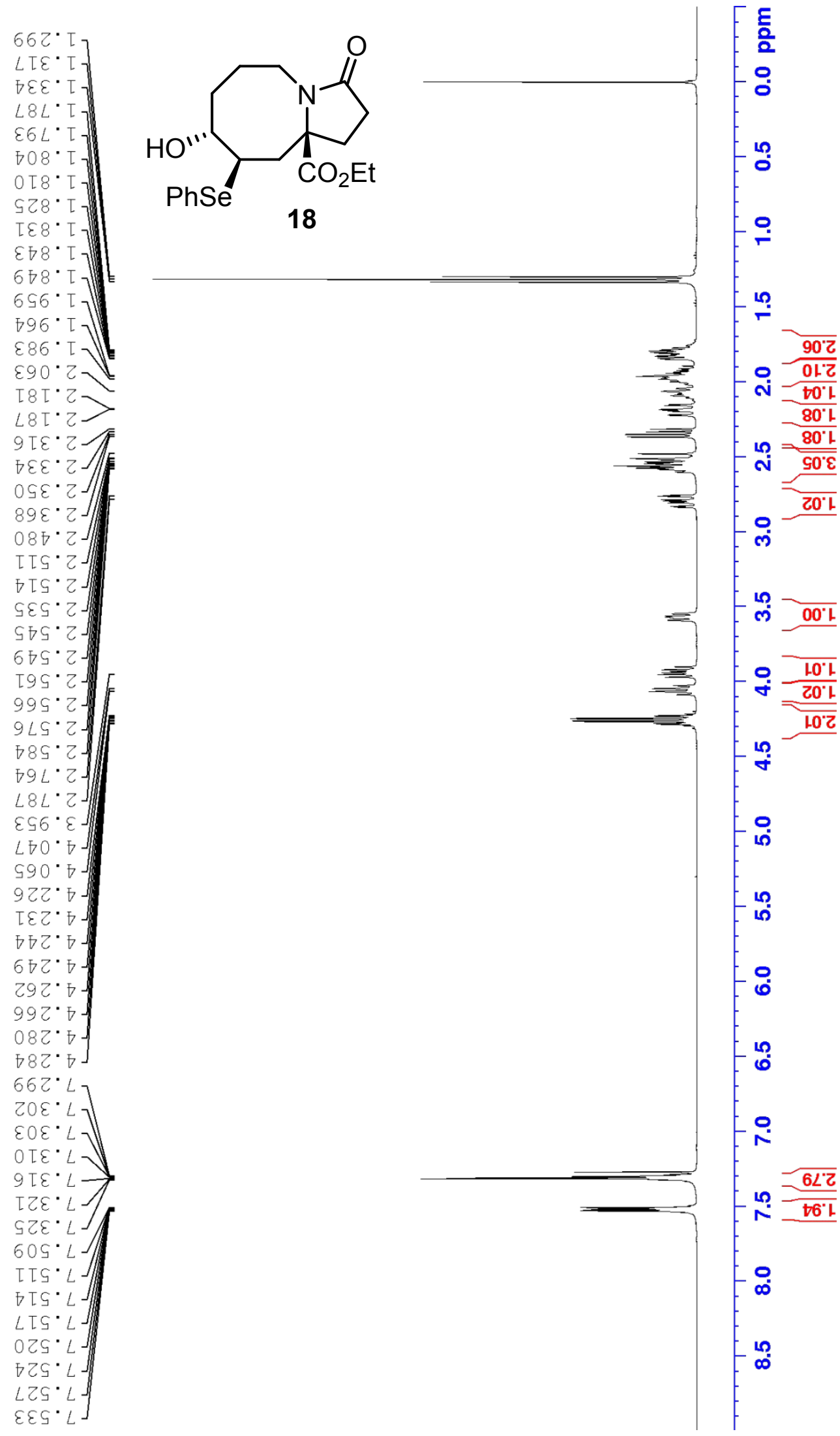

${ }^{13} \mathrm{C}-\mathrm{NMR}$ of Compound $18\left(100 \mathrm{MHz}, \mathrm{CDCl}_{3}\right.$ ) 


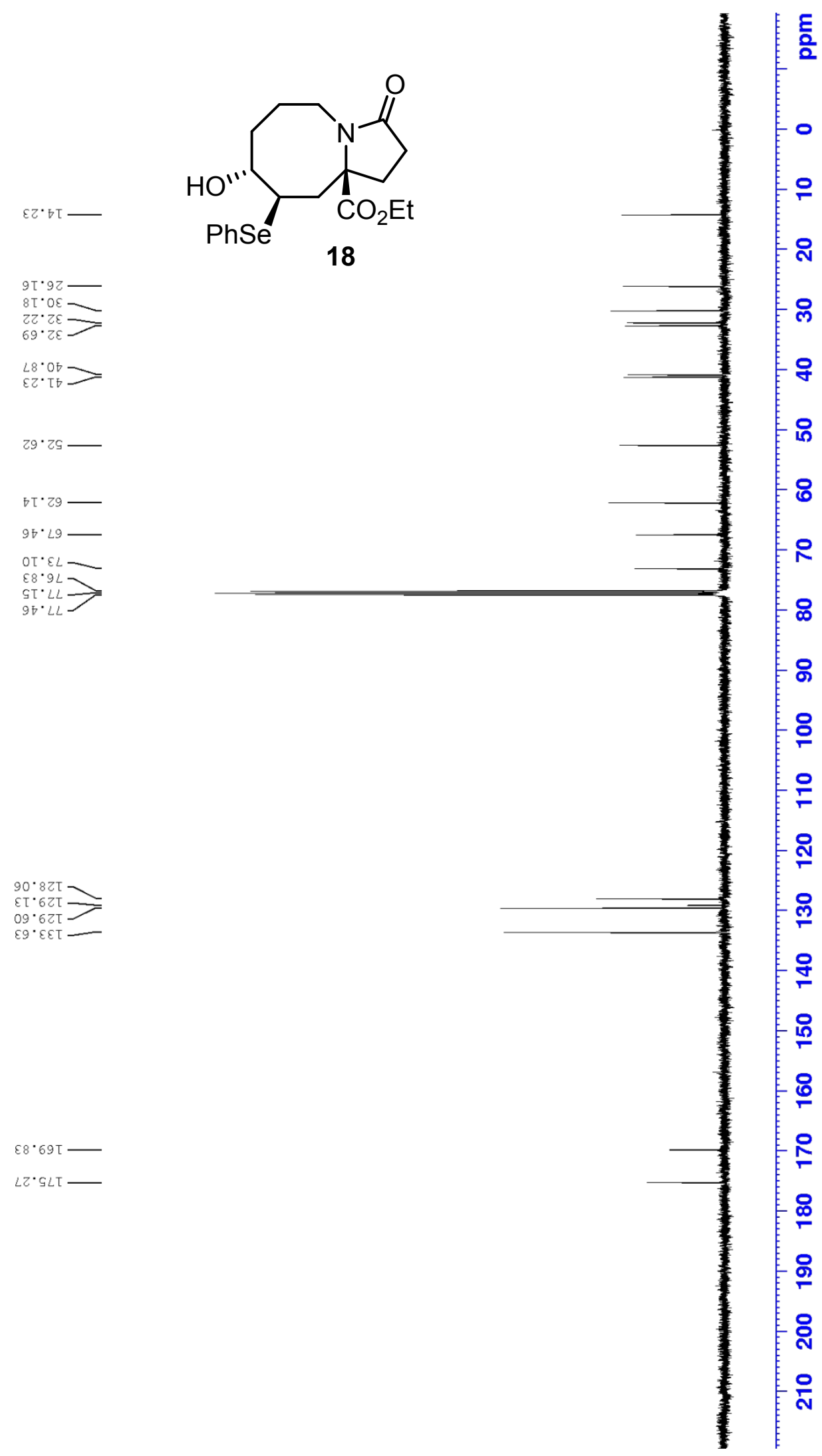

${ }^{1} \mathrm{H}-\mathrm{NMR}$ of Compound (+)-19 (400 $\mathrm{MHz}^{\mathrm{CDCl}}{ }_{3}$ )

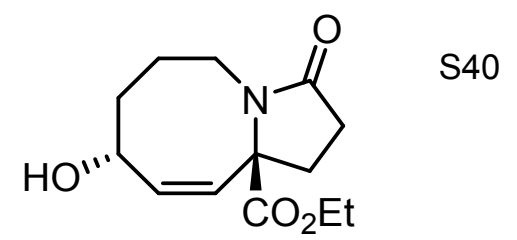




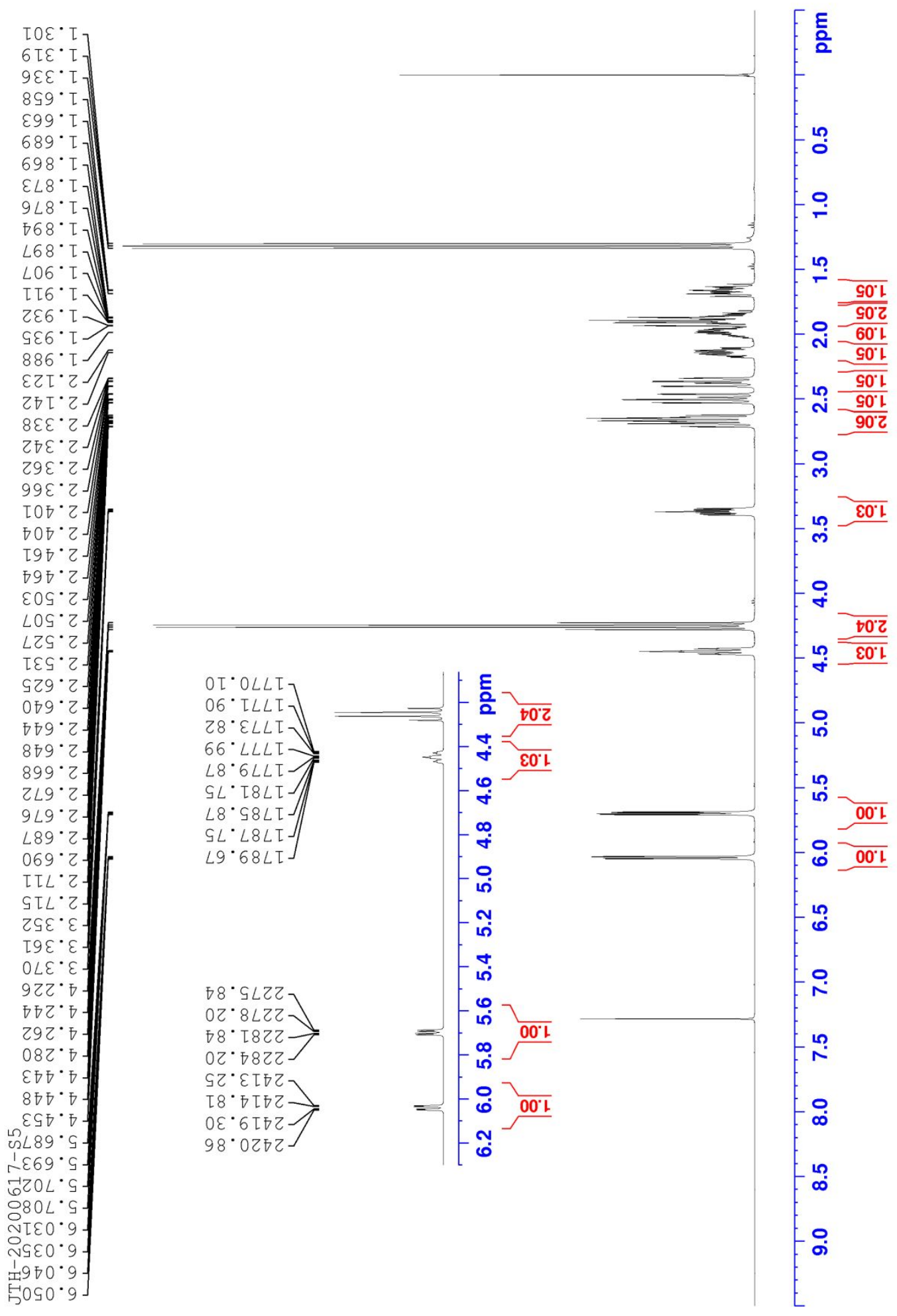

${ }^{13} \mathrm{C}-\mathrm{NMR}$ of Compound (+)-19 (100 $\left.\mathrm{MHz}^{\mathrm{CDCl}}{ }_{3}\right)$ 


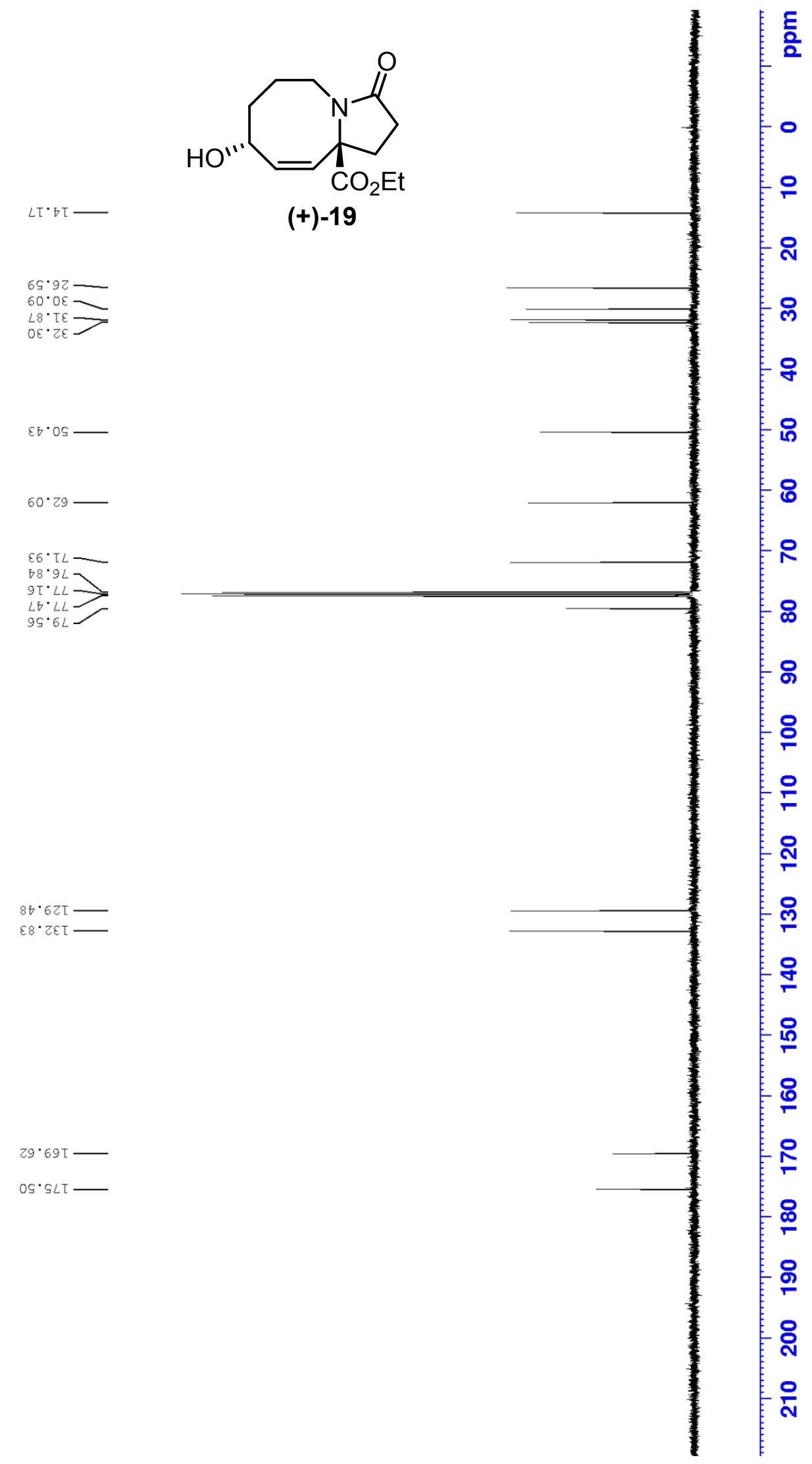

${ }^{1} \mathrm{H}-\mathrm{NMR}$ of Compound (+)-20 (400 $\mathrm{MHz} \mathrm{CDCl}_{3}$ ) 


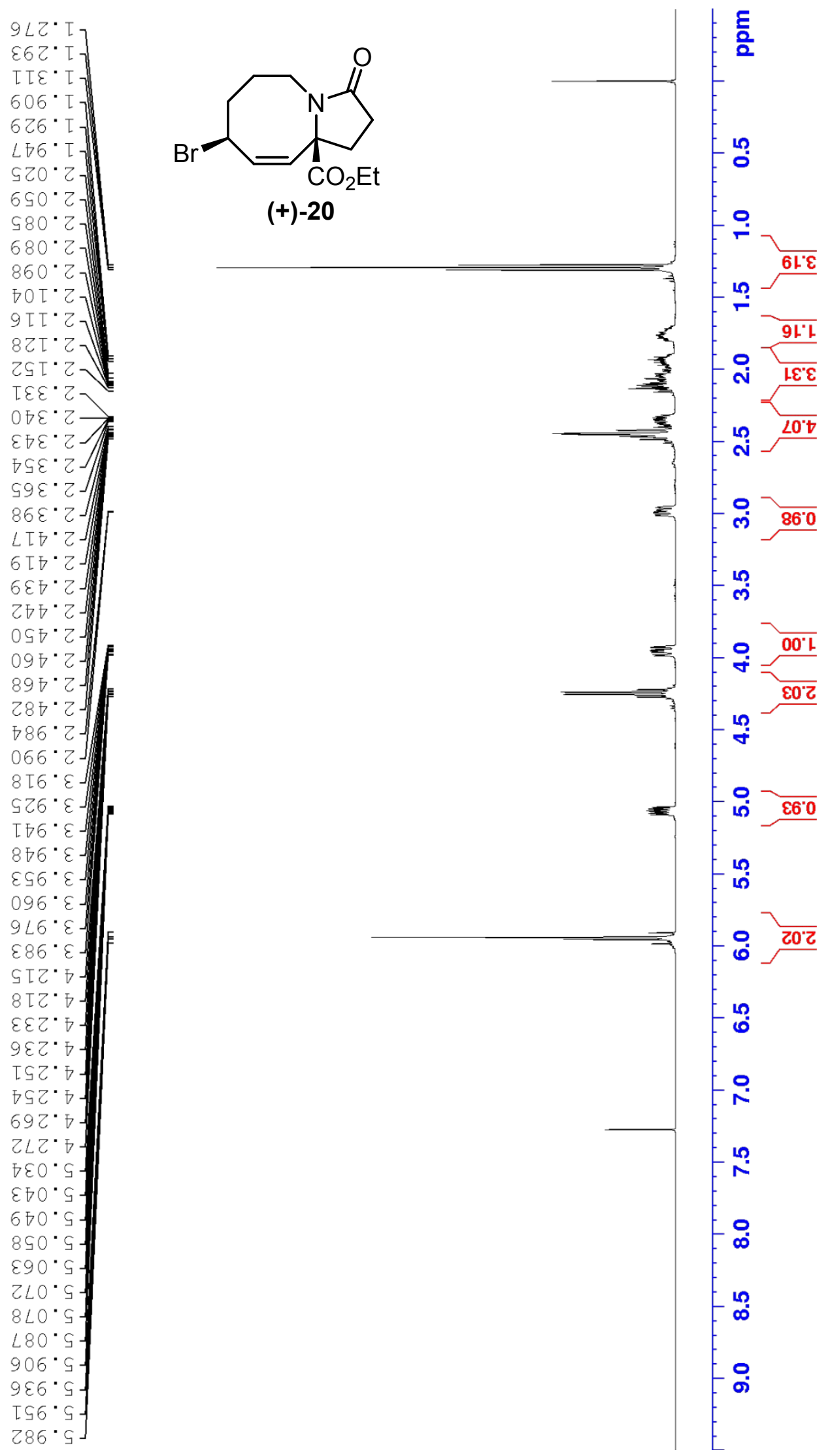

${ }^{13} \mathrm{C}-\mathrm{NMR}$ of Compound (+)-20 (100 $\mathrm{MHz}, \mathrm{CDCl}_{3}$ ) 

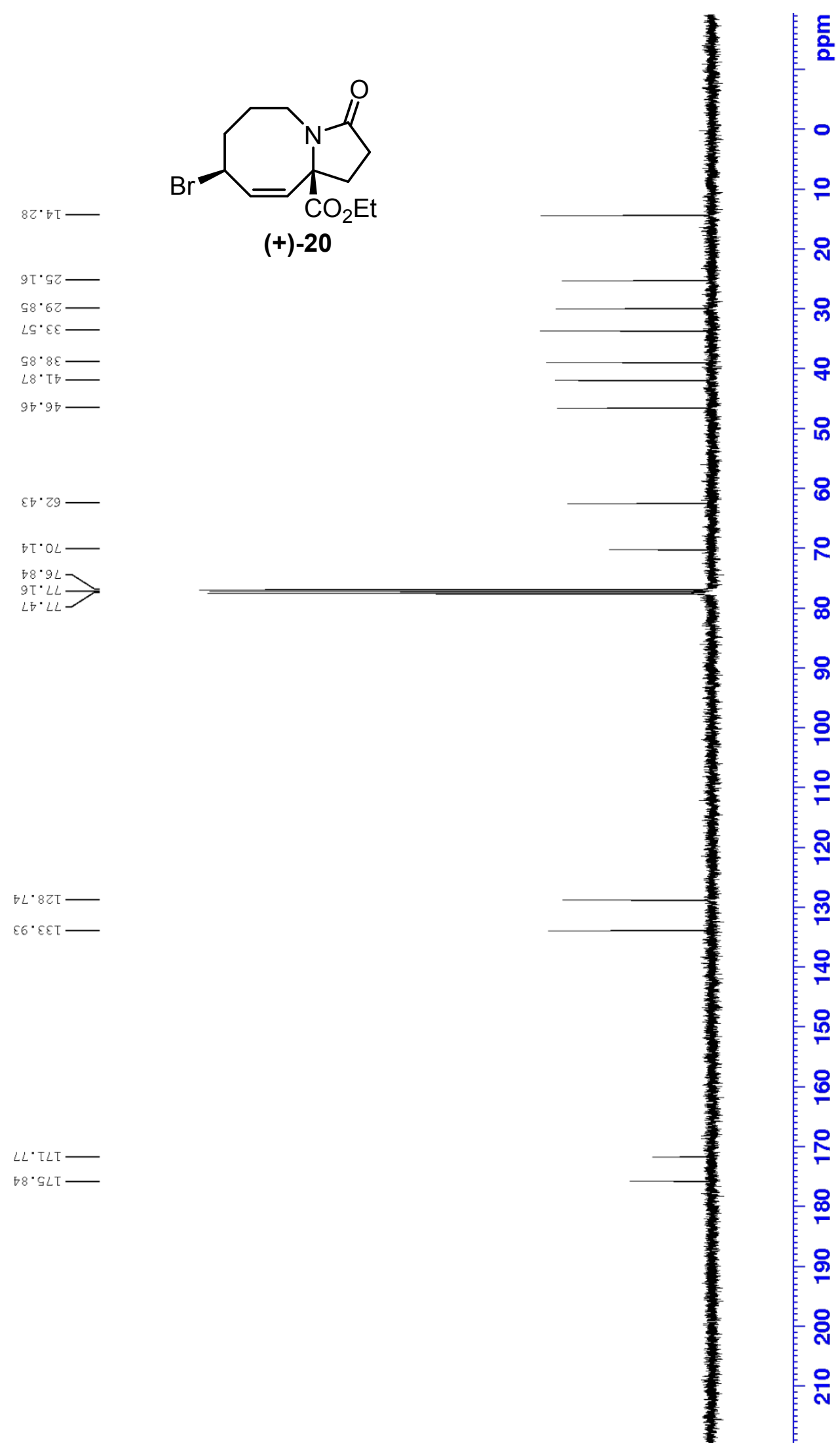

${ }^{1} \mathrm{H}-\mathrm{NMR}$ of Compound $22\left(400 \mathrm{MHz}, \mathrm{CDCl}_{3}\right)$ 


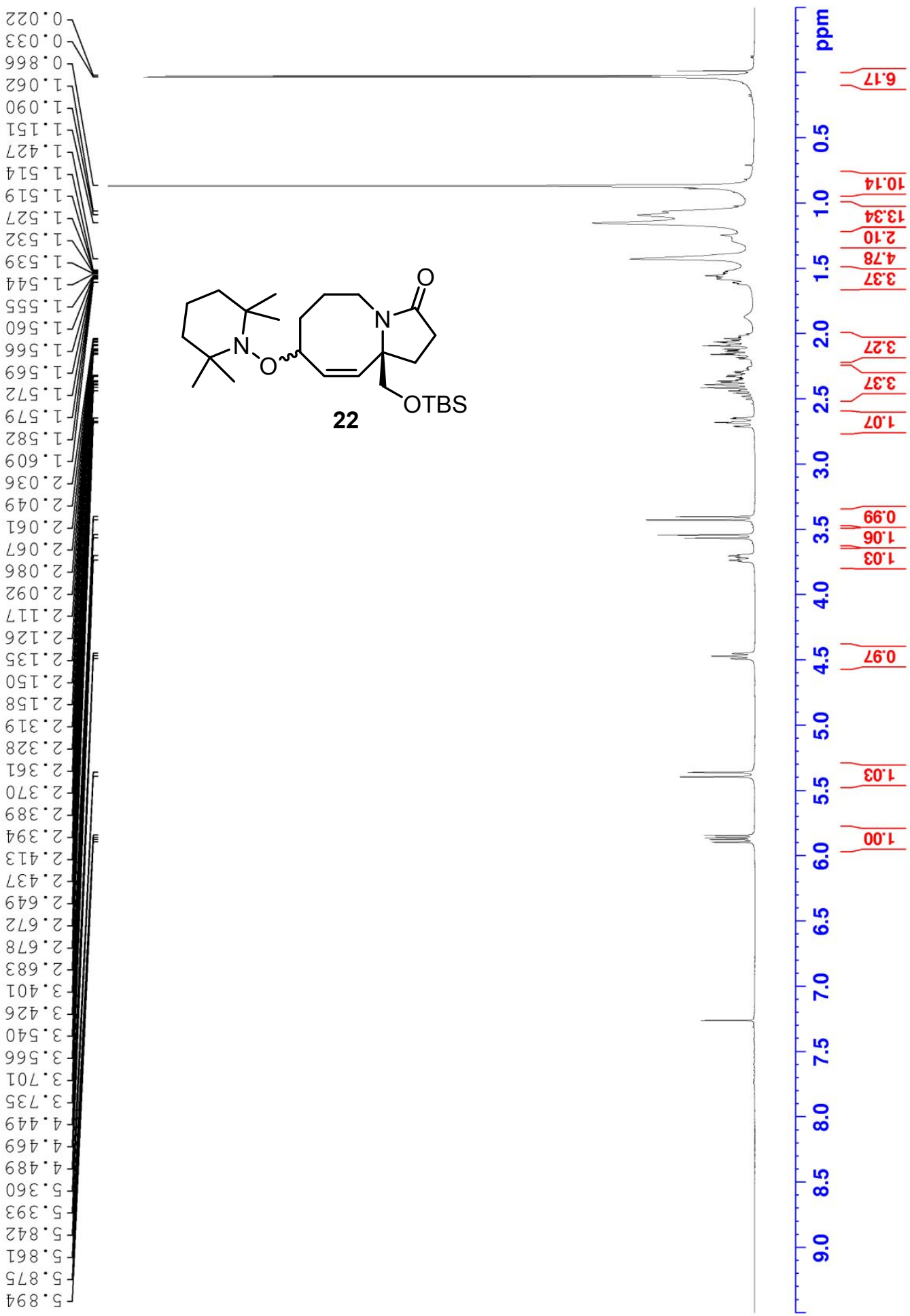

${ }^{13} \mathrm{C}-\mathrm{NMR}$ of Compound $22\left(100 \mathrm{MHz}, \mathrm{CDCl}_{3}\right.$ ) 


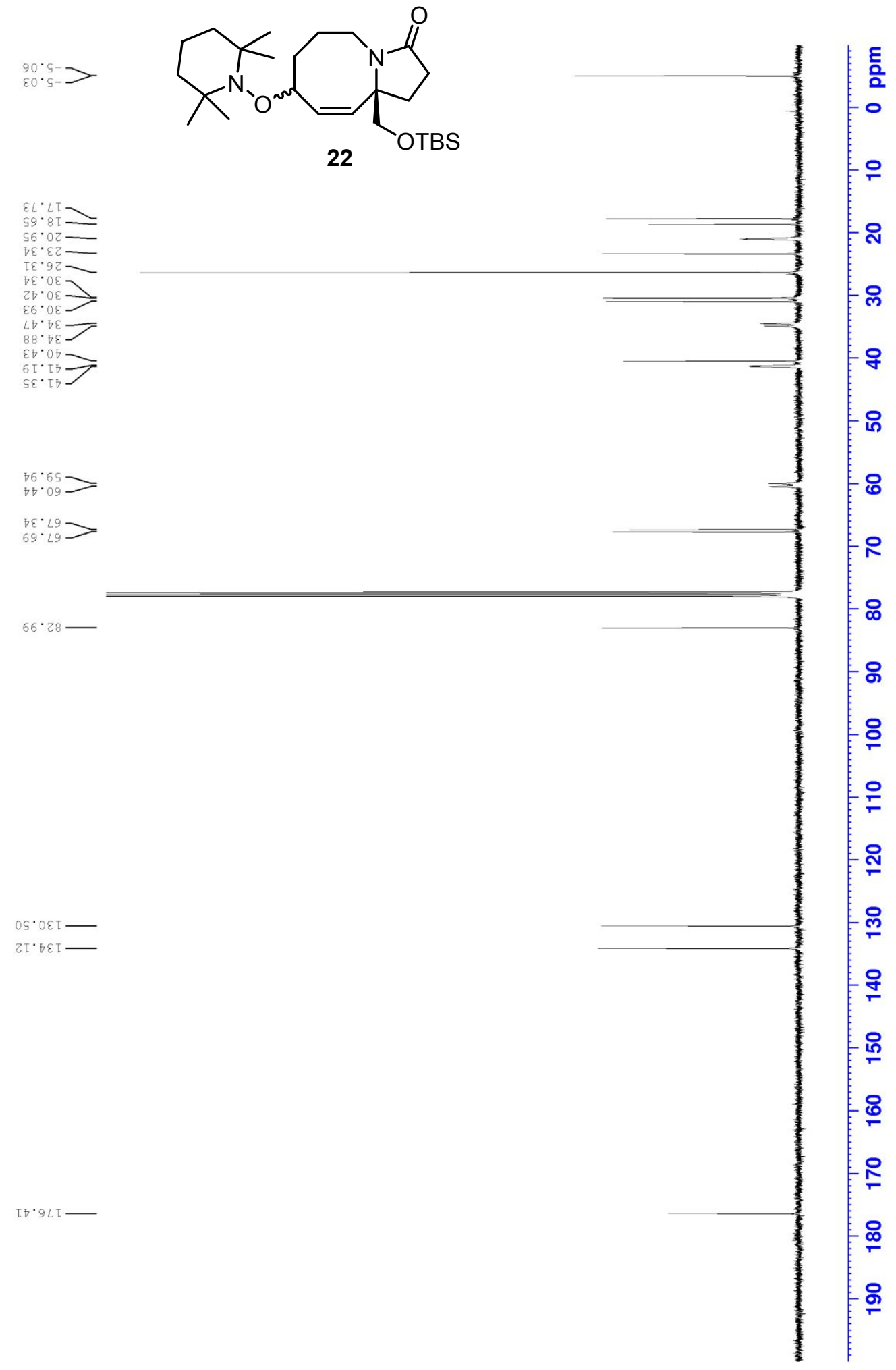

${ }^{1} \mathrm{H}-\mathrm{NMR}$ of Compound $23\left(400 \mathrm{MHz}^{\mathrm{CDCl}}{ }_{3}, 55^{\circ} \mathrm{C}\right)$

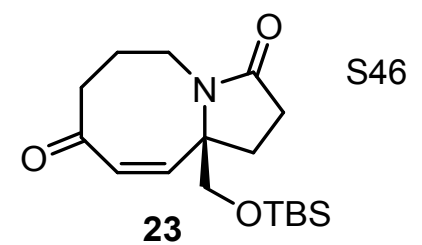




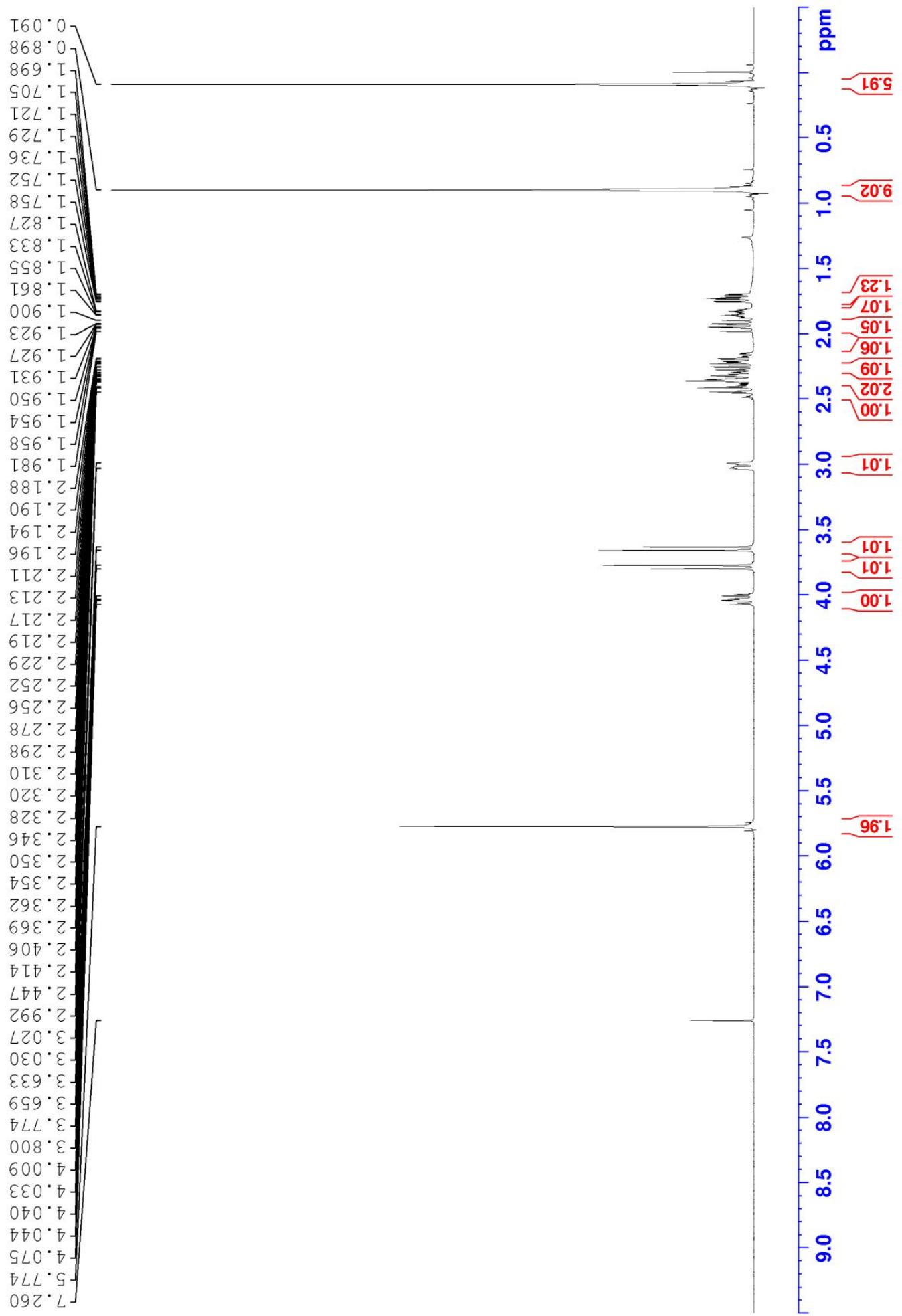

${ }^{13} \mathrm{C}-\mathrm{NMR}$ of Compound 23 (100 $\mathrm{MHz}, \mathrm{CDCl}_{3}, 55^{\circ} \mathrm{C}$ ) 


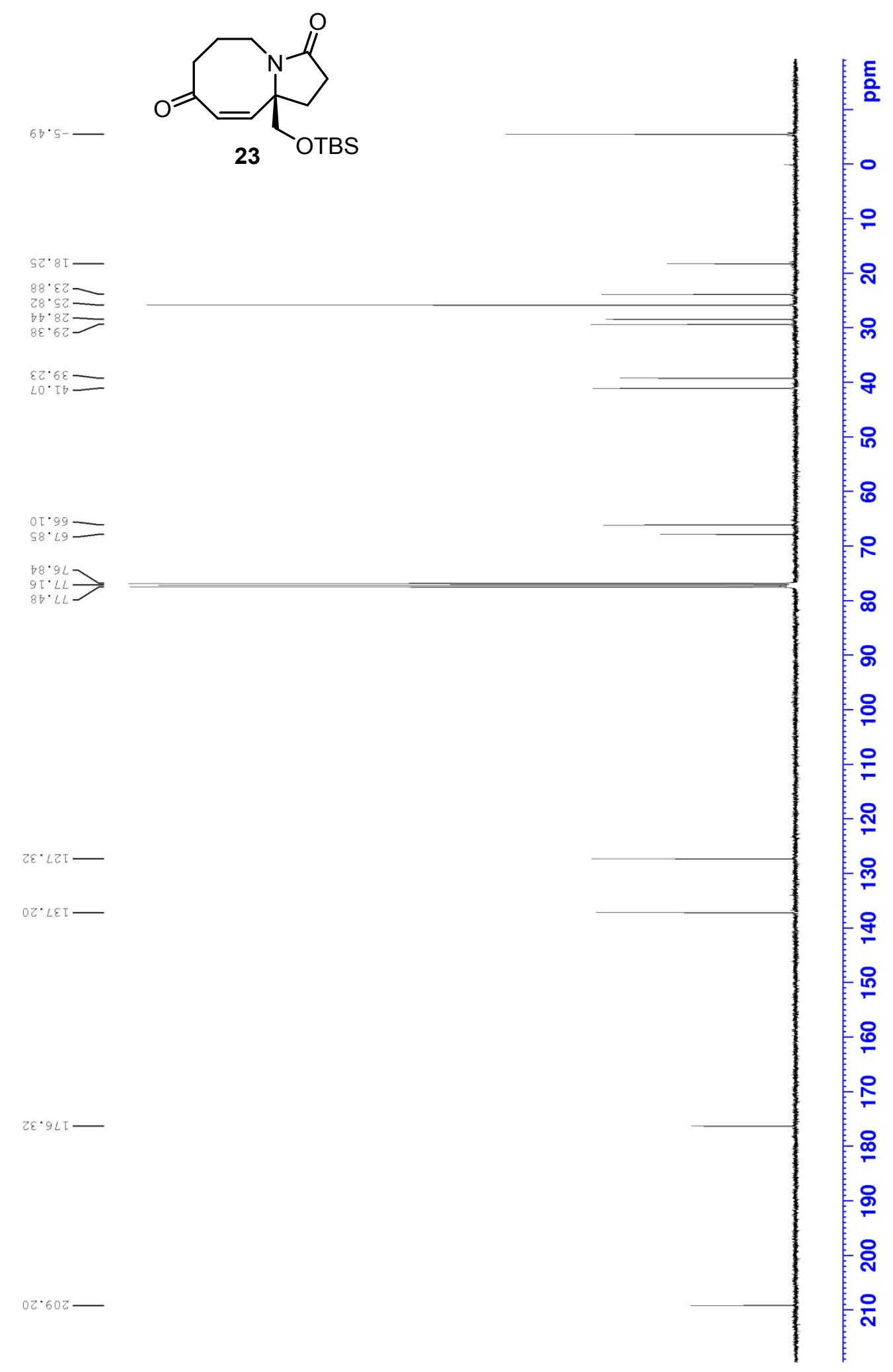

${ }^{1} \mathrm{H}-\mathrm{NMR}$ of Compound $24\left(400 \mathrm{MHz}, \mathrm{CDCl}_{3}\right)$

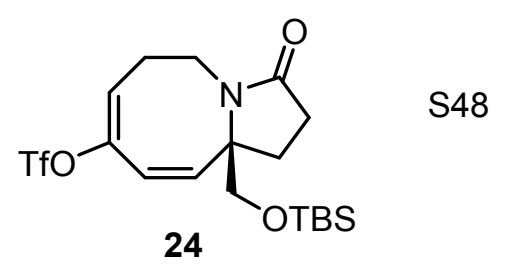




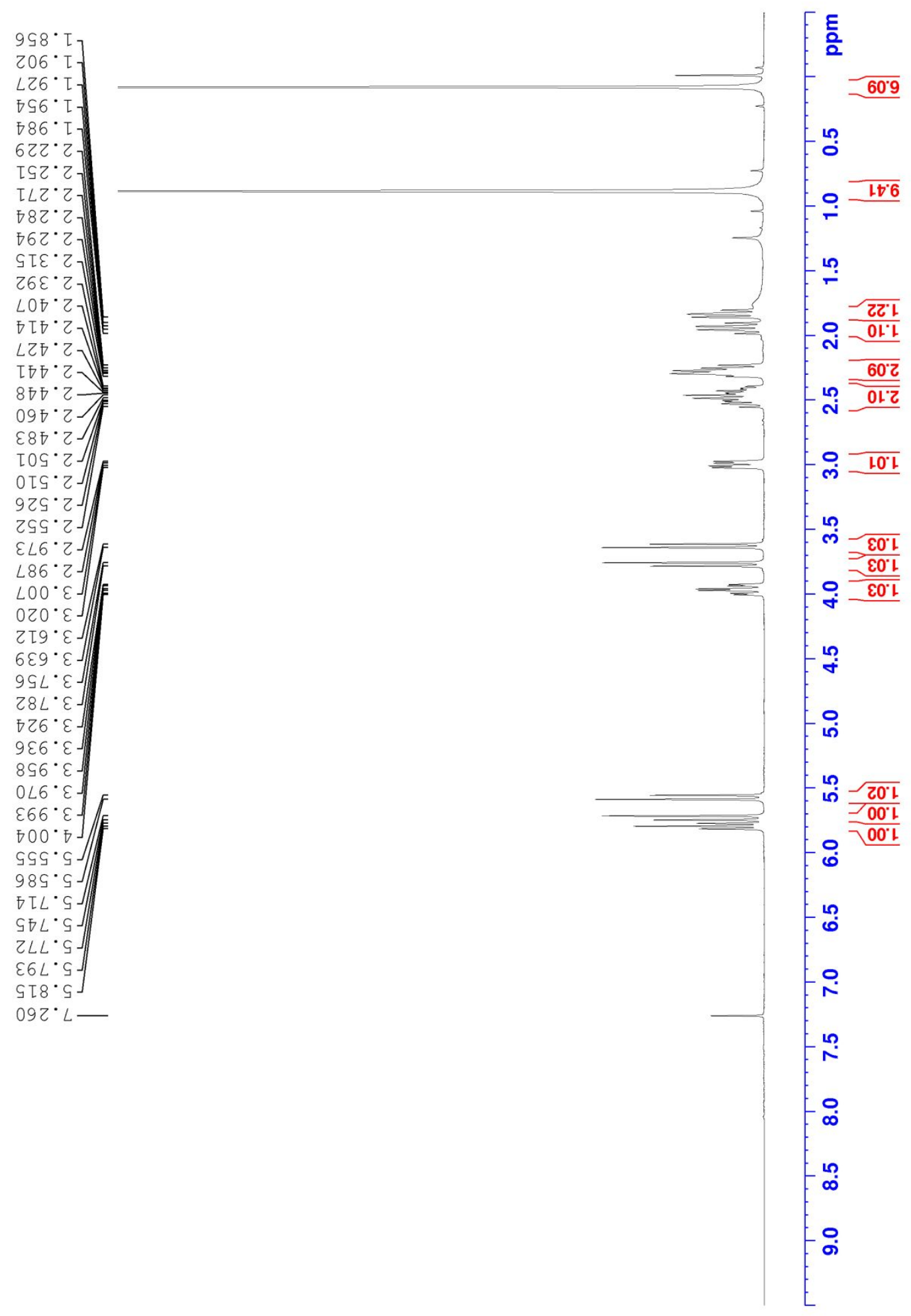

${ }^{13} \mathrm{C}-\mathrm{NMR}$ of Compound $24\left(100 \mathrm{MHz}, \mathrm{CDCl}_{3}\right.$ ) 


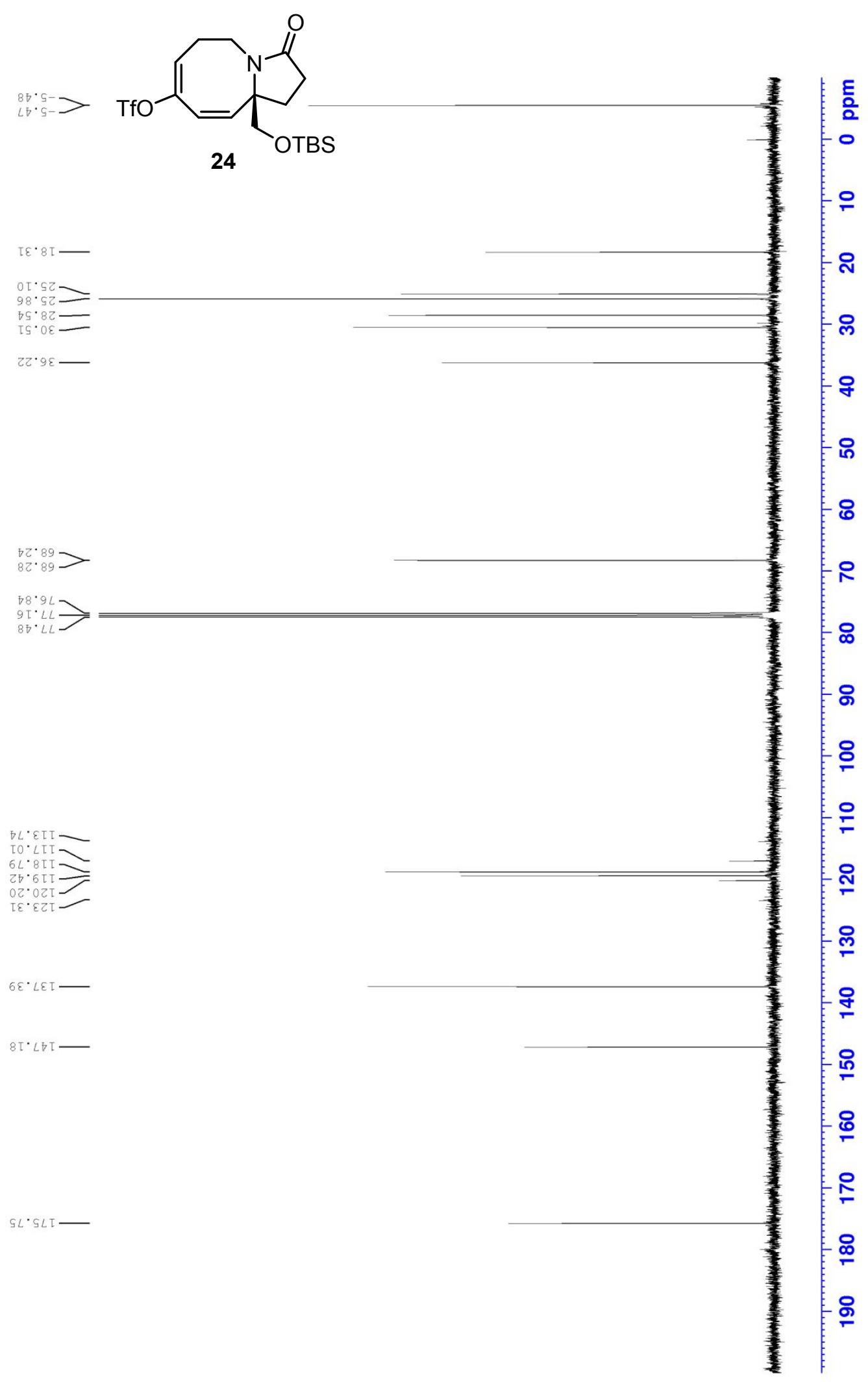

${ }^{1} \mathrm{H}-\mathrm{NMR}$ of Compound $27\left(400 \mathrm{MHz}, \mathrm{CDCl}_{3}\right)$ 


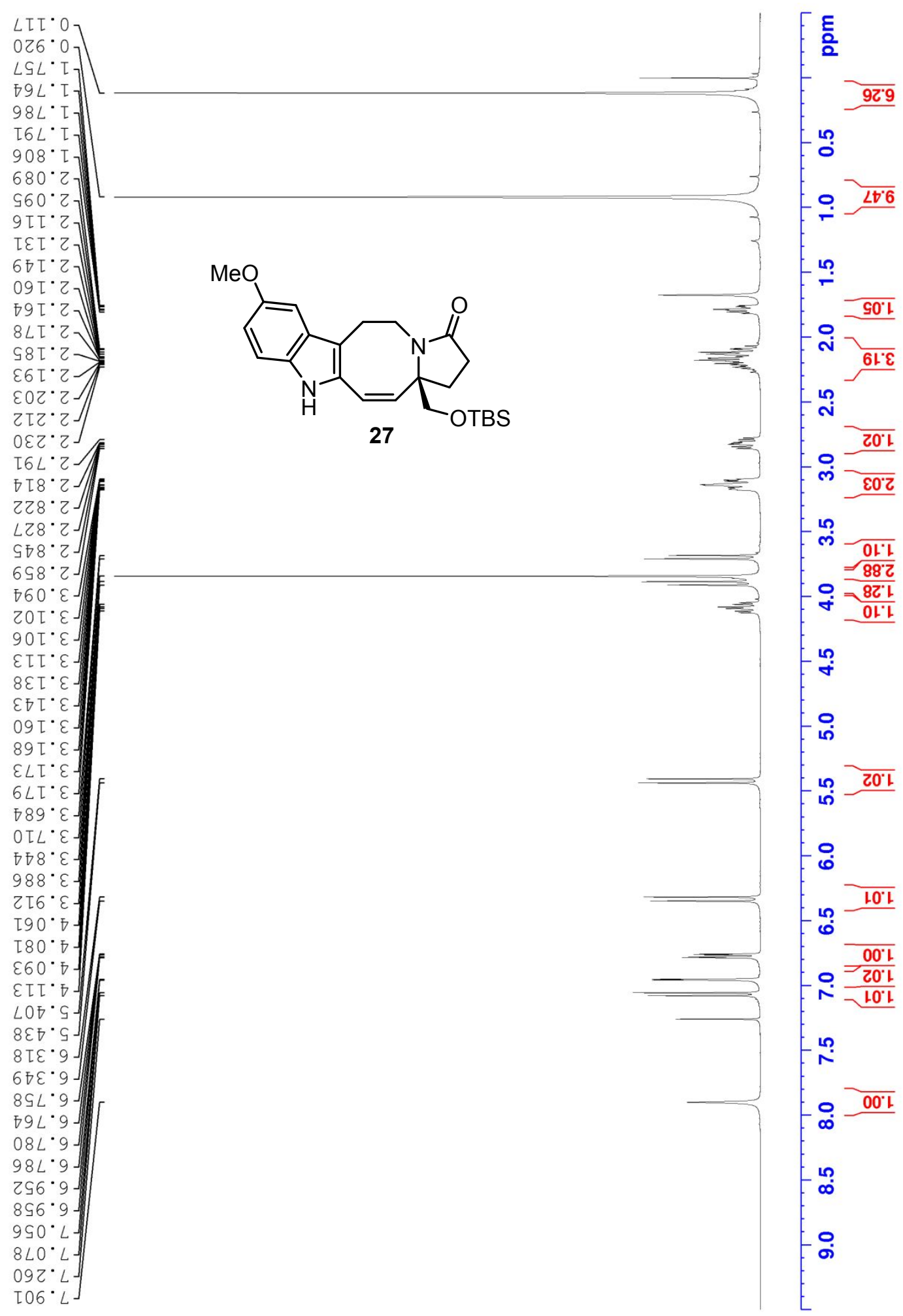

${ }^{13} \mathrm{C}-\mathrm{NMR}$ of Compound $27\left(100 \mathrm{MHz}, \mathrm{CDCl}_{3}\right.$ ) 


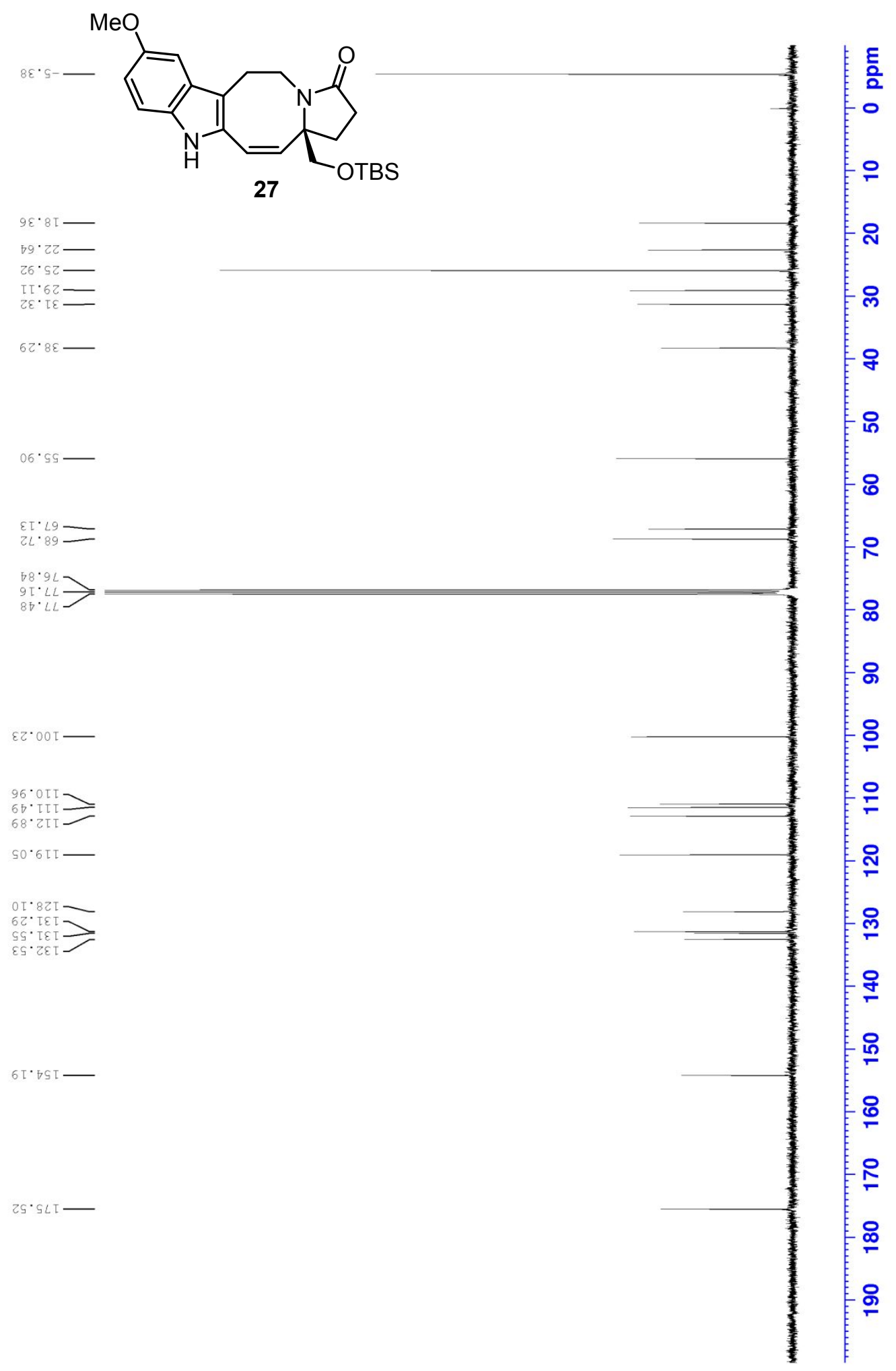

${ }^{1} \mathrm{H}-\mathrm{NMR}$ of Compound $28\left(400 \mathrm{MHz}, \mathrm{CDCl}_{3}\right)$ 


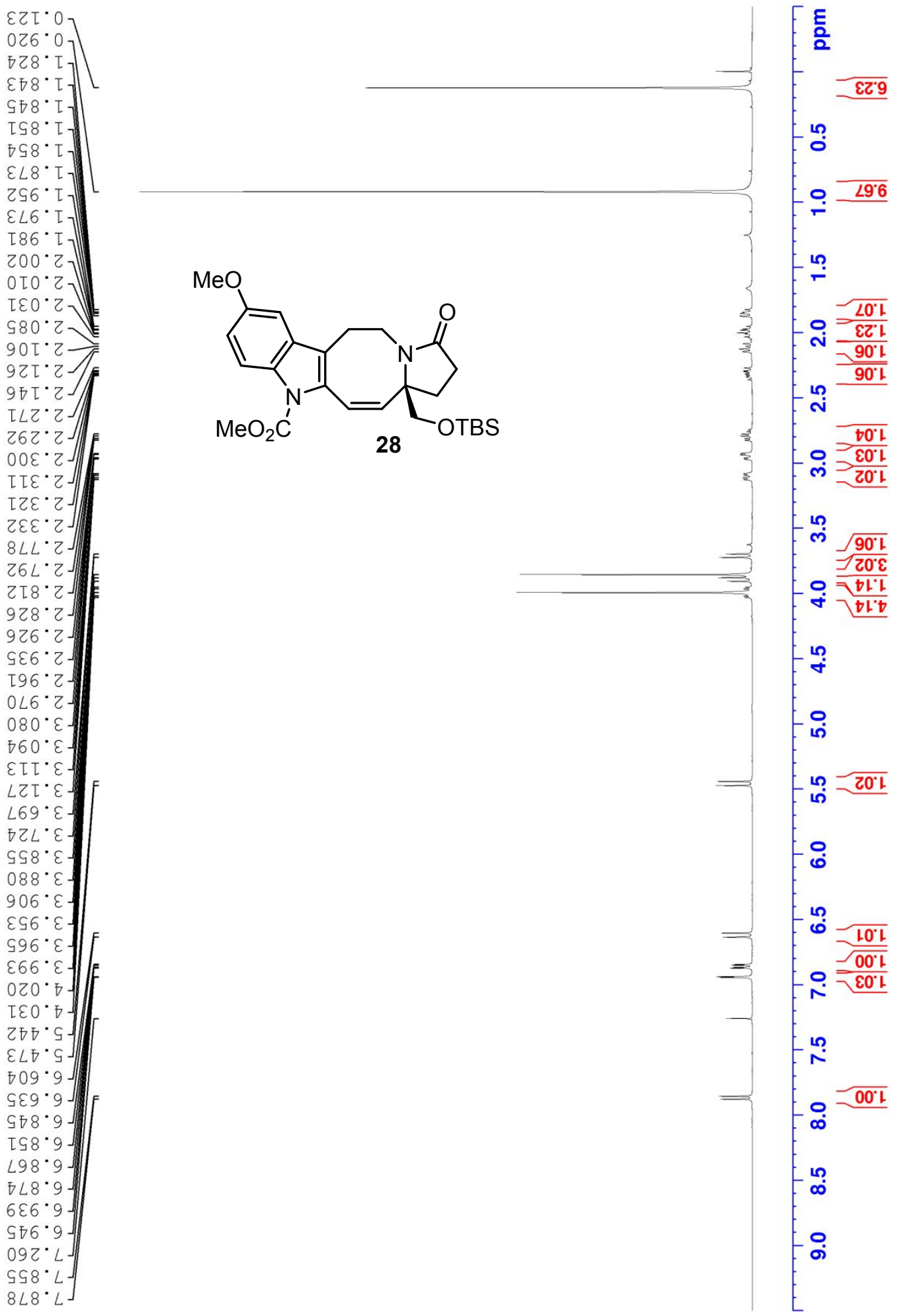

${ }^{13} \mathrm{C}-\mathrm{NMR}$ of Compound $28\left(100 \mathrm{MHz}, \mathrm{CDCl}_{3}\right.$ ) 


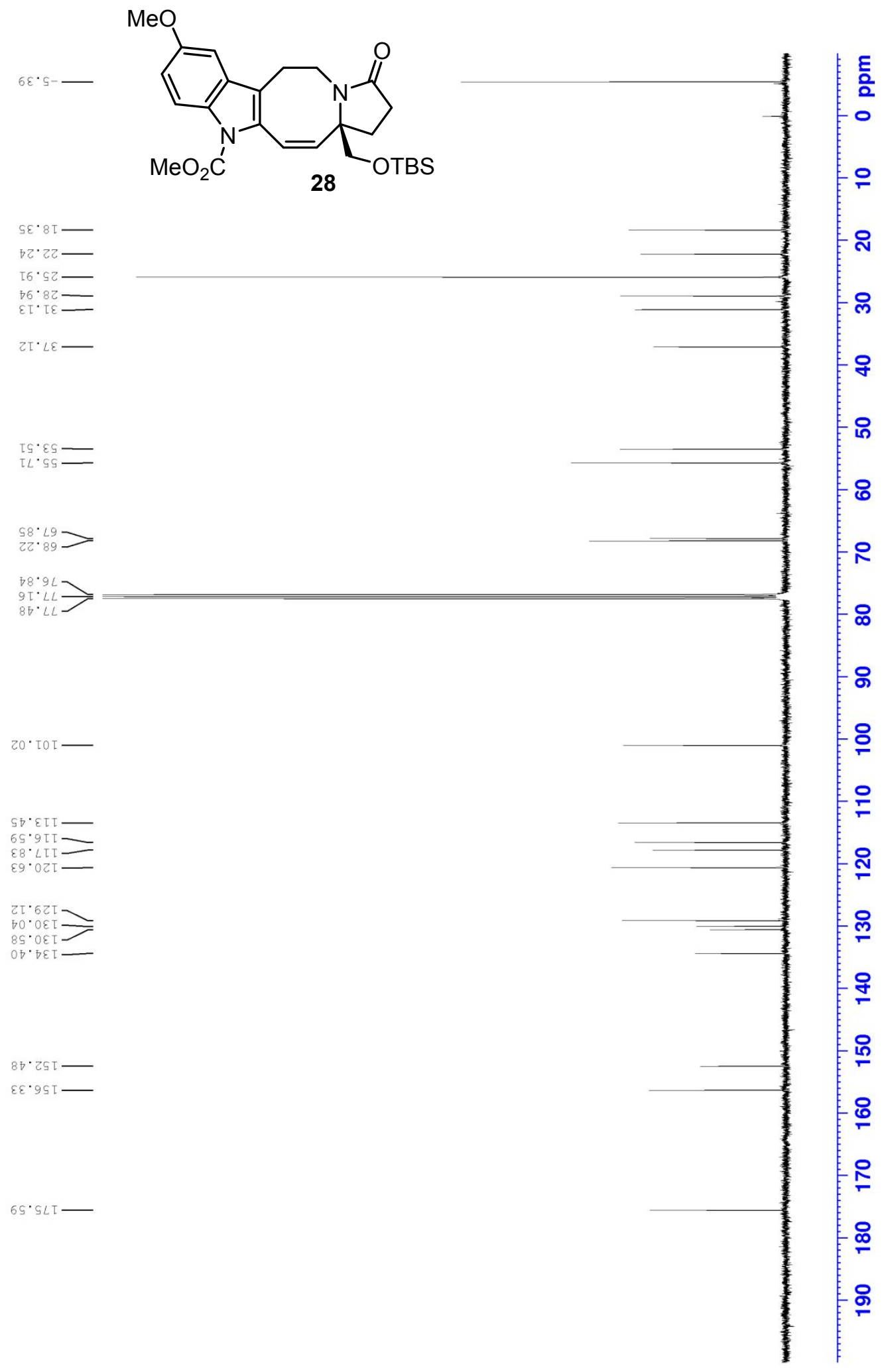

${ }^{1} \mathrm{H}-\mathrm{NMR}$ of Compound $29\left(400 \mathrm{MHz}, \mathrm{CDCl}_{3}\right)$ 

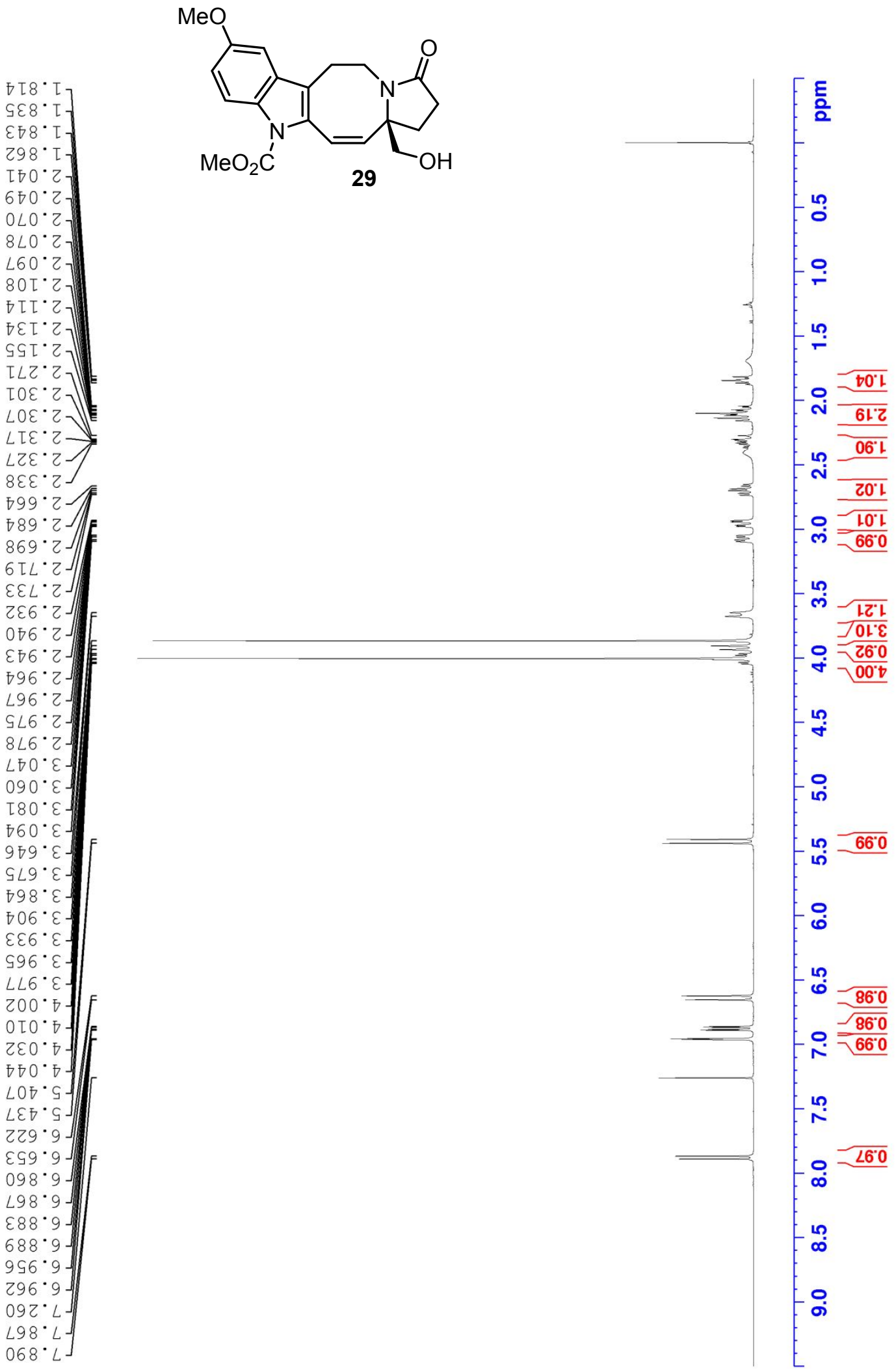

${ }^{13} \mathrm{C}-\mathrm{NMR}$ of Compound $29\left(100 \mathrm{MHz}^{\mathrm{CDCl}}{ }_{3}\right.$ ) 


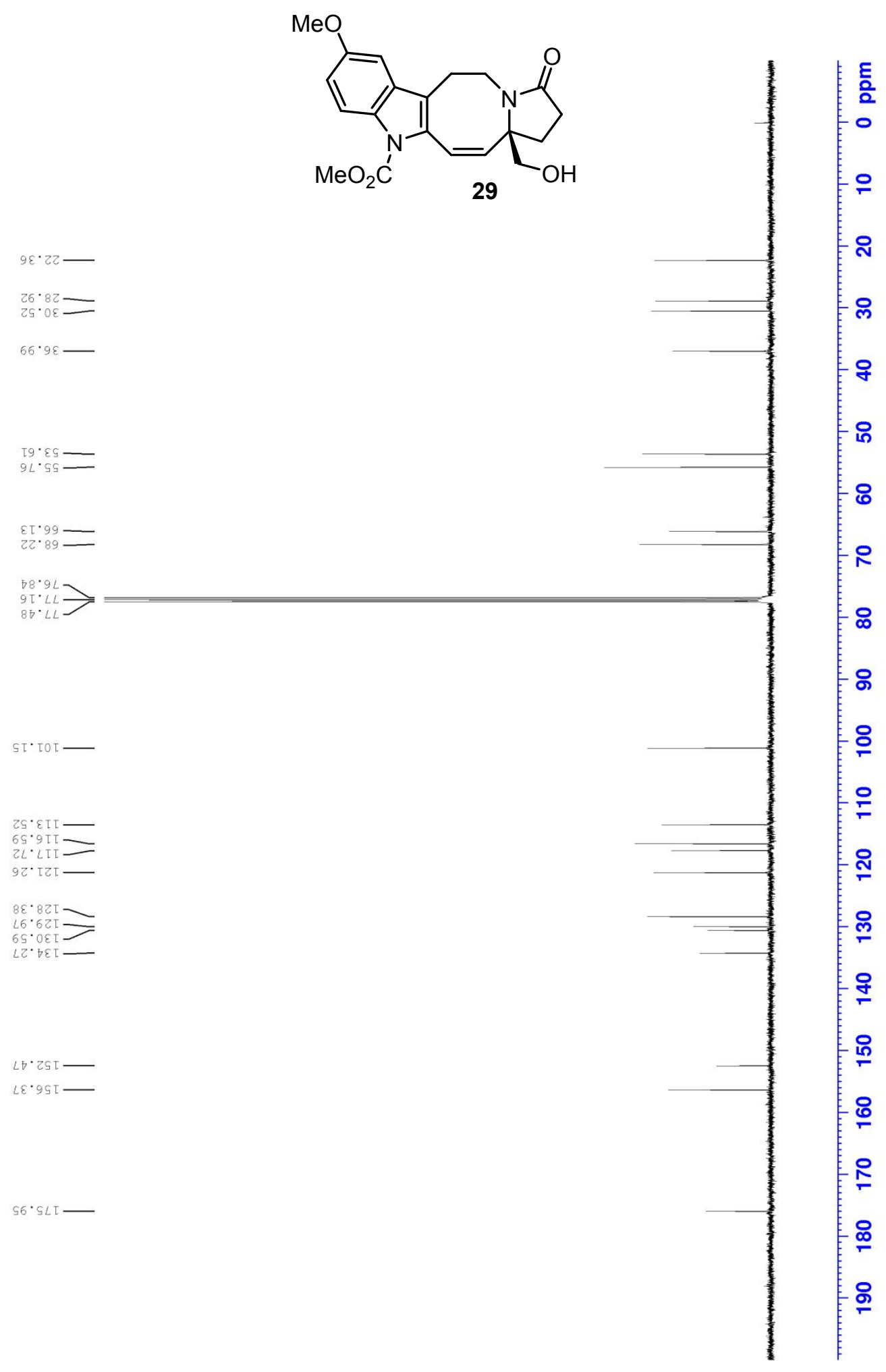




\section{Computational Information}

\section{Computational Details}

All calculations were carried out using density functional theory (DFT) ${ }^{14}$ implemented in the Jaguar 9.1 suite of programs. ${ }^{15}$ Geometry optimizations were performed with the B3LYP ${ }^{16-20}$ levels of theory including Grimme's D3 dispersion correction ${ }^{21}$ for all calculations. The $6-31 \mathrm{G}^{* *}$ basis set $^{22}$ is used for all atoms except for potassium, copper, palladium, and cesium described by the Los Alamos LACVP basis set ${ }^{23-25}$ which includes relativistic effective core potentials for palladium and cesium. The electronic energies of optimized structures were reevaluated with the triple- $\zeta$ basis set cc-pVTZ(-f) ${ }^{26,27}$ The potassium, copper, palladium, and cesium were treated with LACV3P basis set to match the quality of triple- $\zeta$ basis set of main group elements. Analytical vibrational frequencies within harmonic oscillator approximation were calculated at the same level of theory as the geometry optimization to obtain the zero-point vibrational energies and vibrational entropy corrections. Solvation energies were obtained using the self-consistent reaction field (SCRF) ${ }^{28-30}$ approach based on accurate numerical solutions of the Poisson-Boltzmann equation with the 6-31G** and LACVP basis set at the optimized gas-phase geometry with the dielectric constant of $\varepsilon=2.379$ for toluene. The solvation energies are subject to the empirical parametrization of the atomic radii that are used to generate the solute surface for the continuum models. Transition states were located by using the quadratic synchronous transit search (QST) method and confirmed by the presence of only one imaginary frequency. The solution phase Gibbs free energies $G_{(s o l)}$ were computed as follows:

$$
\begin{gathered}
G_{(\text {sol) }}=G(\text { gas })+G(\text { solv }) \\
G(\text { gas })=H(\text { gas })-T S(\text { gas }) \\
H(\text { gas })=E(S C F)+Z P E \\
\Delta G_{(\text {sol) }}=\Sigma G_{(\text {sol) }} \text { for products }-\Sigma G_{(\text {sol) }} \text { for reactants }
\end{gathered}
$$

$G_{(s o l)}$ is the solvation corrected Gibbs free energy from gas phase free energy $G($ gas); $H($ gas) is the enthalpy in gas phase; $\mathrm{T}$ is the temperature at reaction conditions; S(gas) is the entropy in the gas phase; $\mathrm{E}(\mathrm{SCF})$ is the self-consistent field converged electronic energy; ZPE is the vibrational zero-point energy. Note that entropy here refers specifically to the vibrational, rotational, and translational entropy of the solute(s). The solvent entropies are implicitly included in the continuum solvation model.

\section{Single Electron Transfer Mechanism for $\mathrm{Cu}(\mathrm{I}) / \mathrm{Cu}(\mathrm{II}) \mathrm{Cycle}$}

Type I-Marcus Model

Stepwise Bond Dissociation SET<smiles>OC1=CCCC1</smiles>

$(0.00)$

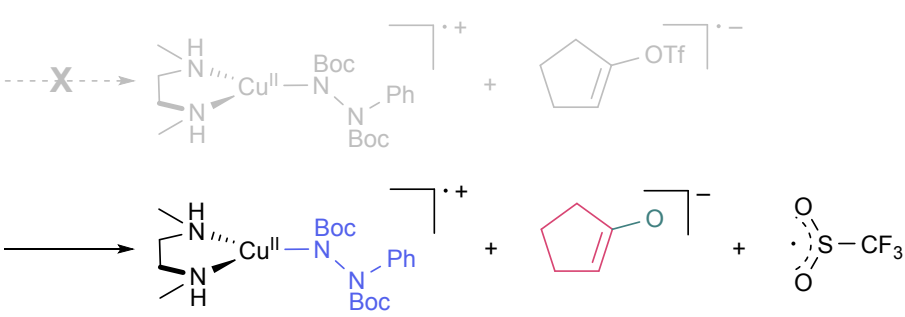

(69.45)

Type II - Saveant Model Concerted Bond Dissociation SET<smiles>O=[Nb]N([PH3+])C1NCCN1</smiles>

$(0.00)$<smiles>[O]C1=CCCC1</smiles>

10a

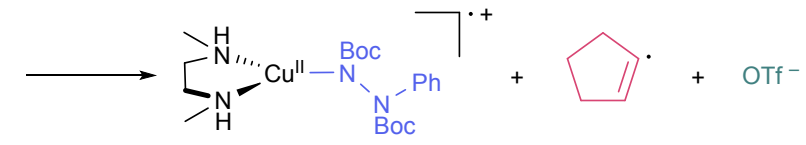

(59.11)

Figure S5. Feasibility study on single electron transfer mechanism for $\mathrm{Cu}(\mathrm{I}) / \mathrm{Cu}(\mathrm{II})$ cycle. All the Gibbs free energies are in $\mathrm{kcal} / \mathrm{mol}$. 
The possibility of other routes to form the $\mathrm{C}-\mathrm{N}$ coupled products for copper catalyst was evaluated. The $\mathrm{Cu}(\mathrm{I}) / \mathrm{Cu}(\mathrm{II})$ catalytic cycles involving single electron transfer (SET) could be possible via stepwise bond dissociation (Type $I$ ) or concerted bond dissociation (Type II) also known as Marcus and Saveant SET model, respectively. ${ }^{31,32}$ Stepwise mechanism Type I should proceed through a formation of radical anion of cyclic vinyl triflate, but the intermediate is too unstable and spontaneously decomposed to be located at $69.5 \mathrm{kcal} / \mathrm{mol}$ higher than starting materials. ${ }^{33}$ Calculations also indicated that concerted mechanism Type II is thermodynamically inaccessible as the endergonic reaction is calculated to be $59.1 \mathrm{kcal} / \mathrm{mol}$ uphill.

\section{Energy Components}

Table S3. Energy components of optimized structures.

\begin{tabular}{|c|c|c|c|c|}
\hline & $\mathrm{E}(\mathrm{SCF}) /(\mathrm{eV})$ & $\mathrm{ZPE} /(\mathrm{kcal} / \mathrm{mol})$ & $\mathrm{S}(\mathrm{gas}) /(\mathrm{cal} / \mathrm{mol} \cdot \mathrm{K})$ & $\mathrm{G}(\mathrm{solv}) /(\mathrm{kcal} / \mathrm{mol})$ \\
\hline & $\begin{array}{c}\text { B3LYP-D3 } \\
\text { /cc-pVTZ(-f)/LACV3P }\end{array}$ & $\begin{array}{c}\text { B3LYP-D3 } \\
/ 6-31 G^{*} / L A C V P\end{array}$ & $\begin{array}{c}\text { B3LYP-D3 } \\
/ 6-31 \mathrm{G}^{*} / \mathrm{LACVP}\end{array}$ & $\begin{array}{c}\text { B3LYP-D3 } \\
/ 6-31 G^{* *} / \text { LACVP }\end{array}$ \\
\hline 7 & -28163.377 & 244.333 & 162.018 & -5.47 \\
\hline $10 a$ & -31469.275 & 85.561 & 114.722 & -2.55 \\
\hline $10 \mathrm{~b}$ & -32539.783 & 104.184 & 116.797 & -2.76 \\
\hline $10 c$ & -33609.766 & 122.162 & 124.691 & -2.49 \\
\hline $10 \mathrm{~d}$ & -34679.965 & 140.703 & 126.243 & -2.78 \\
\hline $10 \mathrm{e}$ & -35750.066 & 158.655 & 136.505 & -2.52 \\
\hline $11 a$ & -33447.754 & 305.407 & 187.036 & -4.22 \\
\hline $11 b$ & -34518.184 & 323.663 & 189.363 & -4.43 \\
\hline $11 \mathrm{c}$ & -35588.191 & 341.736 & 196.121 & -4.34 \\
\hline $11 d$ & -36658.313 & 360.725 & 198.240 & -3.99 \\
\hline $11 \mathrm{e}$ & -37728.473 & 378.622 & 202.712 & -4.23 \\
\hline A & -53780.215 & 469.571 & 255.466 & -23.38 \\
\hline B1 & -85250.570 & 556.637 & 311.724 & -22.03 \\
\hline B1-TS & -85249.898 & 555.202 & 311.264 & -24.51 \\
\hline B5 & -89531.336 & 629.200 & 330.669 & -21.39 \\
\hline B5-TS & -89531.203 & 628.333 & 329.872 & -21.95 \\
\hline C1 & -59079.035 & 538.609 & 274.003 & -6.04 \\
\hline C1-TS & -59078.113 & 537.850 & 273.310 & -5.00 \\
\hline $\mathrm{C2}$ & -60149.336 & 556.812 & 275.051 & -5.97 \\
\hline
\end{tabular}




\begin{tabular}{|c|c|c|c|c|}
\hline C2-TS & -60148.418 & 556.572 & 275.027 & -4.69 \\
\hline C3 & -61219.371 & 574.917 & 287.428 & -5.97 \\
\hline C3-TS & -61218.418 & 574.650 & 279.495 & -4.63 \\
\hline C4 & -62289.617 & 593.489 & 287.186 & -5.90 \\
\hline C4-TS & -62288.492 & 592.985 & 283.765 & -4.47 \\
\hline C5 & -63359.715 & 611.921 & 289.429 & -5.81 \\
\hline C5-TS & -63358.328 & 611.164 & 287.529 & -4.36 \\
\hline D & -25630.521 & 233.779 & 135.713 & -2.03 \\
\hline$E$ & -40813.602 & 344.361 & 213.130 & -9.52 \\
\hline F1 & -72283.844 & 431.875 & 269.101 & -6.73 \\
\hline F1-TS & -72282.500 & 430.030 & 272.138 & -7.04 \\
\hline F2-TS & -73353.117 & 448.435 & 275.171 & -7.39 \\
\hline F3-TS & -74423.180 & 466.656 & 281.009 & -7.15 \\
\hline F4-TS & -75493.508 & 484.697 & 284.933 & -7.09 \\
\hline F5 & -76564.773 & 504.688 & 290.646 & -6.55 \\
\hline F5-TS & -76563.664 & 502.895 & 289.469 & -7.12 \\
\hline G1 & -72283.805 & 431.493 & 270.525 & -9.19 \\
\hline G1-TS & -72283.789 & 431.316 & 265.160 & -8.88 \\
\hline G5 & -76564.680 & 504.359 & 285.578 & -8.91 \\
\hline G5-TS & -76564.516 & 504.002 & 282.711 & -8.75 \\
\hline $\mathbf{H}$ & -38836.285 & 125.744 & 138.901 & -9.42 \\
\hline I1 & -5298.287 & 65.411 & 70.494 & -0.34 \\
\hline 12 & -6368.899 & 83.775 & 74.921 & -0.79 \\
\hline 13 & -7438.997 & 101.816 & 81.050 & -0.77 \\
\hline 14 & -8509.160 & 120.132 & 85.387 & -0.72 \\
\hline 15 & -9579.269 & 138.138 & 91.949 & -0.67 \\
\hline J & -28145.582 & 236.270 & 159.066 & -4.96 \\
\hline $\mathrm{Cs}_{2} \mathrm{CO}_{3}$ & -8265.257 & 9.543 & 96.254 & -37.18 \\
\hline $\mathrm{CsHCO}_{3}$ & -7740.357 & 17.392 & 80.903 & -22.01 \\
\hline
\end{tabular}




\begin{tabular}{ccccc} 
Cs-OTf & -26712.457 & 17.476 & 105.724 & -21.31 \\
$\mathrm{~K}_{3} \mathrm{PO}_{4}$ & -19785.506 & 10.738 & 104.588 & -48.51 \\
$\mathrm{~K}_{2} \mathrm{HPO}_{4}$ & -19035.162 & 17.562 & 96.520 & -38.40 \\
K-OTf & -26937.268 & 17.759 & 96.878 & -20.53 \\
OTf - & -26171.496 & 17.083 & 85.415 & -35.40 \\
OTf & -26166.600 & 15.351 & 92.715 & -0.99 \\
\hline
\end{tabular}




\section{Cartesian Coordinates}

Table S4. Cartesian coordinates of optimized structures in the standard XYZ format.

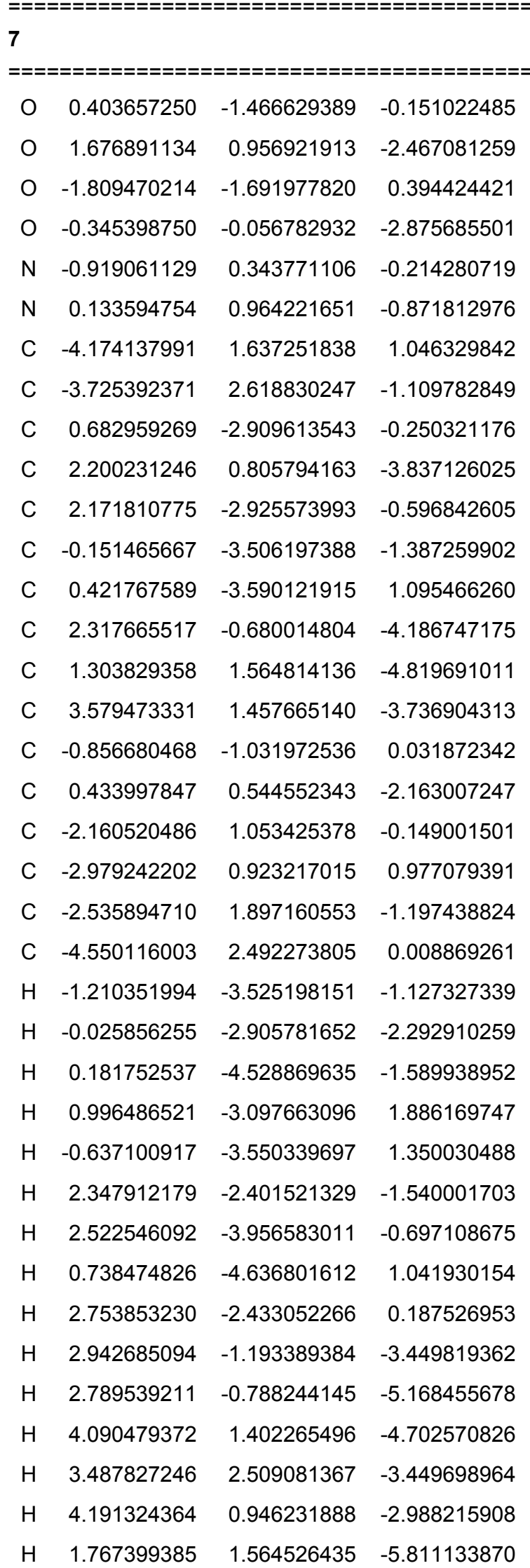

H $\quad 1.186719679 \quad 2.603773638 \quad-4.496187979$

H $\quad 1.335069513 \quad-1.151355886 \quad-4.213010293$

H $\quad 0.319243178 \quad 1.101737331 \quad-4.890409676$

H $\quad 0.927207490 \quad 1.191405603 \quad-0.285780863$

H $\quad-2.685124233 \quad 0.256814768 \quad 1.778476982$

H $\quad-1.908357439 \quad 1.970905683 \quad-2.077062936$

H $\quad-5.478447190 \quad 3.052598463 \quad 0.070698072$

H $\quad-4.808544437 \quad 1.529622071 \quad 1.921541106$

H $\quad-4.012763793 \quad 3.272982524 \quad-1.928092630$ 


$\begin{array}{rrrr}\text { H } & -0.678775497 & -0.842873021 & -0.356557869 \\ \text { H } & 0.036854296 & -0.840379175 & 2.288549105 \\ \text { H } & 0.699876622 & 2.906894866 & 0.531275504 \\ \text { H } & -0.965398155 & 2.427769495 & 0.200329151 \\ \text { H } & 1.613566676 & 1.112936932 & -0.738454250 \\ \text { S } & -1.596853693 & 2.332679800 & 3.457603893 \\ \text { C } & -2.126327230 & 0.802926061 & 4.397993657 \\ \text { O } & -1.359102594 & 3.373321604 & 4.437985251 \\ \text { O } & -2.504289533 & 2.486157226 & 2.330933404 \\ \text { O } & -0.121904628 & 1.828118510 & 2.951422513 \\ \text { F } & -3.289562524 & 1.059657680 & 4.994731684 \\ \text { F } & -1.207839618 & 0.498793142 & 5.311108394 \\ \text { F } & -2.272170056 & -0.219060483 & 3.552281902 \\ \text { C } & 0.534482639 & 0.996007178 & -0.599730006 \\ \text { C } & 0.247856607 & -0.399338483 & 0.034031251 \\ \text { C } & 0.094511343 & -0.095378298 & 1.504621140 \\ \text { C } & -0.013297791 & 1.218116066 & 1.687641188 \\ \text { C } & 0.034853058 & 2.042246552 & 0.431986430 \\ \text { H } & 0.063767974 & 1.114256226 & -1.578503963\end{array}$

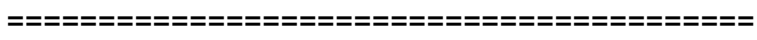

$10 \mathrm{~b}$

\begin{tabular}{crrr}
\multicolumn{4}{c}{$=============================$} \\
C & 0.074665372 & -0.816702751 & -0.589295597 \\
C & -0.962699566 & -0.800194942 & 0.542808208 \\
C & 1.009223120 & 0.401558597 & -0.501885418 \\
C & -1.854335527 & 0.450667975 & 0.449811731 \\
C & 0.251853697 & 1.675041816 & -0.229522844 \\
$\mathrm{C}$ & -1.017178149 & 1.662855117 & 0.172847318 \\
H & -0.448495044 & -0.799237789 & -1.553825837 \\
H & 0.661822219 & -1.740670717 & -0.561633292 \\
H & -0.442834593 & -0.802503287 & 1.509551915 \\
H & -1.584577536 & -1.700544717 & 0.512163517 \\
H & 1.581053044 & 0.511948419 & -1.431773152 \\
H & 1.756494990 & 0.254876870 & 0.291852016 \\
H & -2.409641127 & 0.610999072 & 1.381297521 \\
H & -2.604085282 & 0.345138473 & -0.344487740 \\
H & 0.755887931 & 2.628791247 & -0.354602449 \\
$\mathrm{C}$ & -1.353163119 & 4.390579205 & -1.780873753 \\
$\mathrm{~S}$ & -2.621022583 & 3.573485683 & -0.662763294 \\
$\mathrm{O}$ & -3.276117379 & 2.545073760 & -1.457643106 \\
$\mathrm{O}$ & -3.340142965 & 4.636961757 & 0.010292342
\end{tabular}

$\begin{array}{lrrr}\text { O } & -1.640129168 & 2.914337998 & 0.460838935 \\ \text { F } & -1.984152895 & 5.288467284 & -2.534753424 \\ \text { F } & -0.774194842 & 3.477635603 & -2.561280313 \\ \text { F } & -0.422022464 & 4.997658518 & -1.041629512 \\ \text { C } & 0.074665372 & -0.816702751 & -0.589295597 \\ \text { C } & -0.962699566 & -0.800194942 & 0.542808208 \\ \text { C } & 1.009223120 & 0.401558597 & -0.501885418 \\ \text { C } & -1.854335527 & 0.450667975 & 0.449811731 \\ \text { C } & 0.251853697 & 1.675041816 & -0.229522844 \\ \text { C } & -1.017178149 & 1.662855117 & 0.172847318 \\ \text { H } & -0.448495044 & -0.799237789 & -1.553825837 \\ \text { H } & 0.661822219 & -1.740670717 & -0.561633292 \\ \text { H } & -0.442834593 & -0.802503287 & 1.509551915 \\ \text { H } & -1.584577536 & -1.700544717 & 0.512163517 \\ \text { H } & 1.581053044 & 0.511948419 & -1.431773152 \\ \text { H } & 1.756494990 & 0.254876870 & 0.291852016 \\ \text { H } & -2.409641127 & 0.610999072 & 1.381297521 \\ \text { H } & -2.604085282 & 0.345138473 & -0.344487740 \\ \text { H } & 0.755887931 & 2.628791247 & -0.354602449 \\ \text { C } & -1.353163119 & 4.390579205 & -1.780873753 \\ \text { S } & -2.621022583 & 3.573485683 & -0.662763294 \\ \text { O } & -3.276117379 & 2.545073760 & -1.457643106 \\ \text { O } & -3.340142965 & 4.636961757 & 0.010292342 \\ \text { O } & -1.640129168 & 2.914337998 & 0.460838935 \\ \text { F } & -1.984152895 & 5.288467284 & -2.534753424 \\ \text { F } & -0.774194842 & 3.477635603 & -2.561280313 \\ \text { F } & -0.422022464 & 4.997658518 & -1.041629512\end{array}$

\begin{tabular}{|c|c|c|c|}
\hline \multicolumn{4}{|c|}{$10 c$} \\
\hline C & -3.976036473 & 0.151442964 & -4.969255409 \\
\hline C & -3.117 & .264 & 386 \\
\hline C & 986048 & -1.043 & -4 \\
\hline C & -1.865583689 & 4732 & -4.7 \\
\hline C & -2.012509886 & -1.5141117128 & -5.293833873 \\
\hline C & -0.749563676 & 0.650969357 & -4.918928823 \\
\hline C & -0.773269465 & -0.656346357 & -5.182827976 \\
\hline $\mathrm{H}$ & -4.585 & 571 & $-4.159 c$ \\
\hline $\mathrm{H}$ & -4.682063044 & -0.203406621 & -5.730914382 \\
\hline m & -3.739558337 & 2.156814411 & -5.730444 \\
\hline & & & \\
\hline
\end{tabular}




$\begin{array}{lrrr}\text { H } & -3.873677394 & -1.879639984 & -4.249185023 \\ \text { H } & -2.776531974 & -0.789978174 & -3.423232028 \\ \text { H } & -2.100979752 & 1.802302962 & -3.726935450 \\ \text { H } & -1.472787105 & 2.607080523 & -5.149160012 \\ \text { H } & -2.331280497 & -1.591372512 & -6.343998491 \\ \text { H } & -1.728543743 & -2.530880769 & -4.999649496 \\ \text { H } & 0.188680230 & -1.152938146 & -5.276652280 \\ \text { C } & 2.471084162 & 2.421465591 & -3.502834866 \\ \text { S } & 1.202839703 & 1.066344915 & -3.287830471 \\ \text { O } & 0.541682260 & 1.264637094 & -4.770852590 \\ \text { O } & 0.260739151 & 1.477876692 & -2.254899364 \\ \text { O } & 1.909052848 & -0.203838786 & -3.205655094 \\ \text { F } & 1.857845134 & 3.588964211 & -3.680185668 \\ \text { F } & 3.249664235 & 2.152485296 & -4.547340113 \\ \text { F } & 3.205416953 & 2.462203304 & -2.390787590 \\ \text { C } & -3.976036473 & 0.151442964 & -4.969255409 \\ \text { C } & -3.117809669 & 1.264891453 & -5.594608886 \\ \text { C } & -3.182986048 & -1.043803587 & -4.409542809 \\ \text { C } & -1.865583689 & 1.649314732 & -4.789194706 \\ \text { C } & -2.012509886 & -1.514117128 & -5.293833873 \\ \text { C } & -0.749563676 & 0.650969357 & -4.918928823 \\ \text { C } & -0.773269465 & -0.656346357 & -5.182827976 \\ \text { H } & -4.585347096 & 0.571235975 & -4.159007135 \\ \text { H } & -4.682063044 & -0.203406621 & -5.730914382 \\ \text { H } & -3.739558337 & 2.156814411 & -5.730444093 \\ \text { H } & -2.794281946 & 0.963657519 & -6.598241647 \\ \text { H } & -3.873677394 & -1.879639984 & -4.249185023 \\ \text { H } & -2.776531974 & -0.789978174 & -3.423232028 \\ \text { H } & -2.100979752 & 1.802302962 & -3.726935450 \\ \text { H } & -1.472787105 & 2.607080523 & -5.149160012 \\ \text { H } & -2.331280497 & -1.591372512 & -6.343998491 \\ \text { H } & -1.728543743 & -2.530880769 & -4.999649496 \\ \text { H } & 0.188680230 & -1.152938146 & -5.276652280 \\ \text { C } & 2.471084162 & 2.421465591 & -3.502834866 \\ \text { S } & 1.202839703 & 1.066344915 & -3.287830471\end{array}$

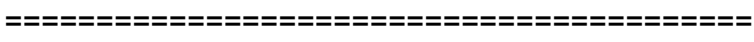

\section{0d}

\begin{tabular}{|c|c|c|c|}
\hline $\mathrm{C}$ & -3.702805183 & -1.202558581 & -2.435711151 \\
\hline C & -2.596045255 & -1.502758299 & -1.393197804 \\
\hline C & -4.897505486 & -0.368279680 & -1.937395221 \\
\hline
\end{tabular}

\begin{tabular}{llll} 
C & -1.323659072 & -0.634374116 & -1.472423180 \\
C & -4.526698267 & 0.973538482 & -1.261465867 \\
C & -1.468537048 & 0.841519818 & -1.038874529 \\
C & -3.587452811 & 1.787338041 & -2.104714857 \\
C & -2.263299459 & 1.690461472 & -1.992647102 \\
H & -4.106861923 & -2.150699979 & -2.809380770 \\
H & -3.252060609 & -0.708338908 & -3.304362347 \\
H & -2.273186630 & -2.542509716 & -1.520697071 \\
H & -3.011686913 & -1.448502266 & -0.376903062 \\
H & -5.482084050 & -0.957121857 & -1.218611164 \\
H & -5.561702021 & -0.172393383 & -2.788831187 \\
H & -0.926253838 & -0.665212291 & -2.495167299 \\
H & -0.558496381 & -1.089702924 & -0.831512228 \\
H & -4.074726261 & 0.767053829 & -0.285011338 \\
H & -5.442514333 & 1.542213262 & -1.065274540 \\
H & -0.473954302 & 1.284471193 & -0.940516377 \\
H & -1.931526078 & 0.883482078 & -0.046233172 \\
H & -3.999754211 & 2.418425568 & -2.885574490 \\
C & -0.205293662 & 4.222876958 & -1.437867258 \\
S & -1.294000396 & 3.981297616 & -2.940778389 \\
O & -0.499812644 & 4.305008522 & -4.109849376 \\
O & -2.563357167 & 4.622287421 & -2.638669792 \\
O & -1.425462312 & 2.357587794 & -2.939064546 \\
F & 0.125805036 & 5.508418994 & -1.347004458 \\
F & 0.896639146 & 3.480267915 & -1.555109547 \\
F & -0.871634674 & 3.852061808 & -0.339291261 \\
C & -3.702805183 & -1.202558581 & -2.435711151 \\
C & -2.596045255 & -1.502758299 & -1.393197804 \\
C & -4.897505486 & -0.368279680 & -1.937395221 \\
C & -1.323659072 & -0.634374116 & -1.472423180 \\
C & -4.526698267 & 0.973538482 & -1.261465867 \\
C & -1.468537048 & 0.841519818 & -1.038874529 \\
C & -3.587452811 & 1.787338041 & -2.104714857 \\
C & -2.263299459 & 1.690461472 & -1.992647102 \\
H & -4.106861923 & -2.150699979 & -2.809380770 \\
\hline & -3.252060609 & -0.708338908 & -3.304362347 \\
\hline
\end{tabular}

C $\quad-1.323659072 \quad-0.634374116-1.472423180$

C $\quad-1.468537048 \quad 0.841519818-1.038874529$

$\begin{array}{llll}\text { C } & -3.587452811 & 1.787338041 & -2.104714857\end{array}$

C $\quad-2.263299459 \quad 1.690461472 \quad-1.992647102$

H $\quad-4.106861923 \quad-2.150699979 \quad-2.809380770$

$\begin{array}{llll}H & -3.252060609 & -0.708338908 & -3.304362347\end{array}$

H $\quad-2.273186630 \quad-2.542509716 \quad-1.520697071$

H $\quad-3.011686913 \quad-1.448502266 \quad-0.376903062$

H $\quad-5.482084050 \quad-0.957121857 \quad-1.218611164$

H $\quad-5.561702021 \quad-0.172393383 \quad-2.788831187$

H $\quad-0.926253838 \quad-0.665212291 \quad-2.495167299$

H $\quad-0.558496381 \quad-1.089702924 \quad-0.831512228$

H $\quad-4.074726261 \quad 0.767053829 \quad-0.285011338$

H $\quad-5.442514333 \quad 1.542213262 \quad-1.065274540$

$\begin{array}{llll}\text { H } & -0.473954302 & 1.284471193 & -0.940516377\end{array}$

$\begin{array}{llll}\text { H } & -1.931526078 & 0.883482078 & -0.046233172\end{array}$

H $\quad-3.999754211 \quad 2.418425568 \quad-2.885574490$

C $\quad-0.205293662 \quad 4.222876958 \quad-1.437867258$

$\begin{array}{lllll}S & -1.294000396 & 3.981297616 & -2.940778389\end{array}$

O $\quad-0.499812644 \quad 4.305008522 \quad-4.109849376$

$\begin{array}{llll}\text { O } & -2.563357167 & 4.622287421 & -2.638669792\end{array}$

F $\quad 0.125805036 \quad 5.508418994-1.347004458$

F $\quad 0.896639146 \quad 3.480267915 \quad-1.555109547$

F $\quad-0.871634674 \quad 3.852061808 \quad-0.339291261$

C $-2.596045255-1.502758299-1.393197804$

C $\quad-4.897505486-0.368279680 \quad-1.937395221$

C $-1.323659072 \quad-0.634374116 \quad-1.472423180$

C $-1.468537048 \quad 0.841519818-1.038874529$

$\begin{array}{llll}\text { C } & -3.587452811 & 1.787338041 & -2.104714857\end{array}$

C $\quad-2.263299459 \quad 1.690461472 \quad-1.992647102$

H $\quad-3.252060609-0.708338908-3.304362347$

H $\quad-2.273186630 \quad-2.542509716 \quad-1.520697071$

H $\quad-3.011686913 \quad-1.448502266 \quad-0.376903062$

H $\quad-5.482084050 \quad-0.957121857 \quad-1.218611164$

H $\quad-5.561702021 \quad-0.172393383 \quad-2.788831187$

H $\quad-0.558496381 \quad-1.089702924 \quad-0.831512228$ 
H $\quad-1.850206455$

C $\quad-0.566713517$

2.055648876

0.765897330

H -0.759355743

0.786757558

H $\quad-0.210937240$

1.770300290

C -0.824126904

0.178681211

H $\quad-0.098080563$

0.603737005

H -1.694036936

$-0.125587156$

C 0.576673649

$-0.251514141$

H 0.167520748

$-0.669363237$

H $\quad-0.436473812$

1.801058882

$-1.685762191$

H -1.850206455

2.055648876

1.913530242

C $\quad-0.566713517$

0.765897330

2.991262334

H $\quad-0.759355743$

0.786757558

1.653412539

C -0.824126904

1.770300290

$-2.091912233$

H $\quad-0.098080563$

0.178681211

0.603737005

$-2.796406740$

H -1.694036936

$-0.125587156$

$-2.686068137$

C $\quad 0.576673649$

$-0.251514141$

1.641070771

$\mathrm{H}$

c

H

c

H$$
\text { H }
$$

H

\section{$s$}

c

\section{0}

0

\section{F}

$F$

\section{$F$}

$\begin{array}{lll}0.167520748 & -1.269583373 & 1.639036943 \\ 1.250897753 & -0.203430816 & 2.503589620\end{array}$

$\begin{array}{lll}1.414044530 & -0.006266081 & 0.357817211\end{array}$

\section{$\begin{array}{lll}2.474730516 & -0.100272761 & 0.621278128\end{array}$}

$\begin{array}{lll}1.298574581 & 1.034559976 & 0.035312696\end{array}$

C $\quad \begin{array}{llll}1.176345087 & -0.960890036 & -0.828662520\end{array}$

H $1.868728459-0.676446916-1.633270770$

H $1.482997769-1.967653391-0.512953921$

C $\quad-0.238737288-1.075586667 \quad-1.420090525$

H $\quad-0.933654737-1.428539111 \quad-0.648853474$

H $\quad-0.208926740 \quad-1.870888833 \quad-2.175394164$

S $\quad-4.662547534 \quad 1.382987234 \quad-0.303437177$

$\begin{array}{llll}-5.791205736 & 0.586986675 & -1.560452282\end{array}$

$\begin{array}{llll}-5.249780268 & 1.198091932 & 1.014952059\end{array}$

$\begin{array}{llll}-4.292850360 & 2.705809956 & -0.789249328\end{array}$

O $\quad-3.442252024 \quad 0.324584545 \quad-0.541841330$

F $\quad-6.932882195 \quad 1.275370029-1.566299070$

F $-6.028267262-0.677750211-1.222900725$

C $\quad \begin{array}{llll}-2.163317809 & 0.752512996 & -0.045785712\end{array}$

$\begin{array}{llll}\text { C } & -1.880908615 & 0.481608572 & 1.227962405\end{array}$

H $\quad-2.663134358 \quad 0.006526333 \quad 1.814187185$

C $\quad-1.290633931 \quad 1.298421615 \quad-1.126526590$

H $\quad-0.436473812 \quad 1.801058882 \quad-0.669363237$
$-1.685762191$

1.913530242

2.991262334

1.653412539

$-2.091912233$

$-2.796406740$

$-2.686068137$

1.641070771

.639036943 


$\begin{array}{rrrr}\text { H } & -0.430752191 & -4.226644401 & 2.263103932 \\ \text { H } & 1.576370268 & -3.575393893 & 0.117531221 \\ \text { H } & 3.502337540 & 2.296100908 & 1.004462879 \\ \text { H } & 4.543340378 & 1.338508565 & 2.075103286 \\ \text { H } & 5.831358607 & 0.469259028 & 0.017080230 \\ \text { H } & 4.925120083 & -0.286536973 & -1.311849805 \\ \text { H } & 4.775123817 & 1.456913256 & -1.015122852 \\ \text { H } & 4.756514137 & -1.271602323 & 1.586933083 \\ \text { H } & 3.804931737 & -1.937543228 & 0.246367312 \\ \text { H } & 2.775957490 & 1.144466547 & 2.147636169 \\ \text { H } & 2.987225340 & -1.364803408 & 1.719469050 \\ \text { H } & 0.200751913 & 2.396678689 & -0.786459632 \\ \text { H } & -3.587282201 & 0.426218206 & -0.257004001 \\ \text { H } & -3.363560483 & 4.721624386 & -0.217404631 \\ \text { H } & -0.900698459 & 4.578145594 & -0.582240345 \\ \text { H } & -4.689673614 & 2.617160591 & -0.076774077 \\ \text { C } & 0.332864104 & -0.334448871 & -4.760373346 \\ \text { C } & 1.544556510 & -1.223871284 & -4.379445146 \\ \text { C } & 1.947185945 & -0.777466314 & -2.953355294 \\ \text { H } & 2.371327073 & -1.153467247 & -5.092429588 \\ \text { H } & 1.222575278 & -2.270491250 & -4.348828517 \\ \text { C } & -0.221723009 & 0.069906153 & -3.415733536 \\ \text { H } & -0.395956194 & -0.867992934 & -5.382001528 \\ \text { H } & 0.642908994 & 0.550032285 & -5.337902041 \\ \text { C } & 0.657648843 & -0.172609867 & -2.434474820 \\ \text { H } & -1.201054001 & 0.511661686 & -3.275435929 \\ \text { H } & 2.294824503 & -1.604141370 & -2.325416488 \\ \text { H } & 2.749355921 & -0.031639619 & -2.964282476\end{array}$

\section{$11 \mathrm{~b}$}

\begin{tabular}{|c|c|c|c|}
\hline $\mathrm{O}$ & -0.396175964 & -2.212263061 & -0.25010651 \\
\hline & 2.549700733 & 0.059372214 & -0.64806102 \\
\hline & -2.421121915 & -1.430342618 & 0.47146942 \\
\hline & 1.060519626 & 0.083838599 & 1.0992459 \\
\hline & -0.959560229 & -0.081584210 & -0.68 \\
\hline & 0.363057823 & 08 & -1 \\
\hline & -1.963 & 3.457770093 & \\
\hline & -3.895494167 & 2.201342865 & -0.8373287 \\
\hline & -0.409299869 & -3.439942789 & 0.0 \\
\hline & 3.755129143 & 0.091679164 & 0.1 \\
\hline
\end{tabular}
C $\quad 0.925802162 \quad-4.084781385$
0.186062585
C $-1.588390147 \quad-4.323384540$
0.147615818
C $\quad-0.437796255 \quad-3.067951876$
2.045840560
C $\quad 3.771874622 \quad 1.369330615$
1.040118116
C $3.818479561-1.174931029$
1.053281367
C $4.876796184 \quad 0.109688671$
$-0.843687776$
C $-1.350413792-1.280852183$
$-0.083310739$
C $1.324309176 \quad 0.070954321$
$-0.090386315$
C -1.765845863
1.094274808
$-0.585871767$
C $-1.184949464 \quad 2.302852830$
$-0.190165913$
C -3.123888719
1.043307850
$-0.914408890$
C $\quad-3.320488981 \quad 3.413648944$
$-0.452041698$
H $\quad-2.534466573-3.835976226$
0.382900270
H $\quad-1.550378101-4.526128920$
$-0.927492077$
H $\quad-1.535118739-5.278735493$
0.679890545
H $\quad 0.334007969-2.321032108$
2.252033616
H $\quad-1.405780264 \quad-2.652739301$
2.328339623
H $\quad 0.974358908 \quad-4.267440745$
$-0.891436854$
H $\quad 1.042481162 \quad-5.038663618$
0.708357801
H $\quad-0.244086731 \quad-3.959681138$
2.650032006
H $1.755515830-3.428938068$
0.464566565
H $\quad 3.650225567 \quad 2.245768811$
0.395914709
H $\quad 4.734228314 \quad 1.453539987$
1.554945971
H $\quad 5.850806615$
0.122983477
$-0.345830586$
H 4.821173960
$-0.777043200$
$-1.481854117$
H 4.794983999
0.996576553
$-1.478511749$
H $4.765877865-1.201411765$
1.600813496
H $\quad 3.765966459 \quad-2.063862838$
0.416646095
H $2.972434623 \quad 1.359758181$
1.780923059
H $2.996244038-1.199075896$
1.768783612
$\begin{array}{lll}H & -0.138751874 & 2.328461684\end{array}$
0.089550852
H $\quad-3.567023843$
0.100385233
$-1.209048594$
H $\quad-3.925252300$
4.314179679
$-0.398648804$
H $\quad-1.506344587$
4.392496269
0.182200284
H $\quad-4.951237825$
2.154809091
$-1.088795104$
C $\quad 1.618333079-1.517738150$
$-4.337473528$
$\begin{array}{llll}\text { C } & 0.743068115 & -0.677324811 & -5.277589342\end{array}$
C $\quad 0.705709708 \quad 0.789029954 \quad-4.817372178$
H $\quad-0.277511460-1.081578619 \quad-5.272842135$
H $\quad 1.105468429 \quad-0.739889411 \quad-6.309648544$
C $1.049471478-1.511465853 \quad-2.911036332$
H $\quad 2.632592889-1.097485768 \quad-4.320022129$ 


$\begin{array}{lrrr}\text { H } & 1.705631101 & -2.546738246 & -4.703319355 \\ \text { C } & 0.514123334 & 0.911601981 & -3.328030166 \\ \text { H } & -0.092627390 & 1.334686126 & -5.336942267 \\ \text { H } & 1.641118439 & 1.296906431 & -5.098530739 \\ \text { C } & 0.650255182 & -0.118541970 & -2.487921188 \\ \text { H } & 1.783067652 & -1.898209516 & -2.196009477 \\ \text { H } & 0.176701110 & -2.172864767 & -2.832041045 \\ \text { H } & 0.243745913 & 1.882969440 & -2.921414706\end{array}$

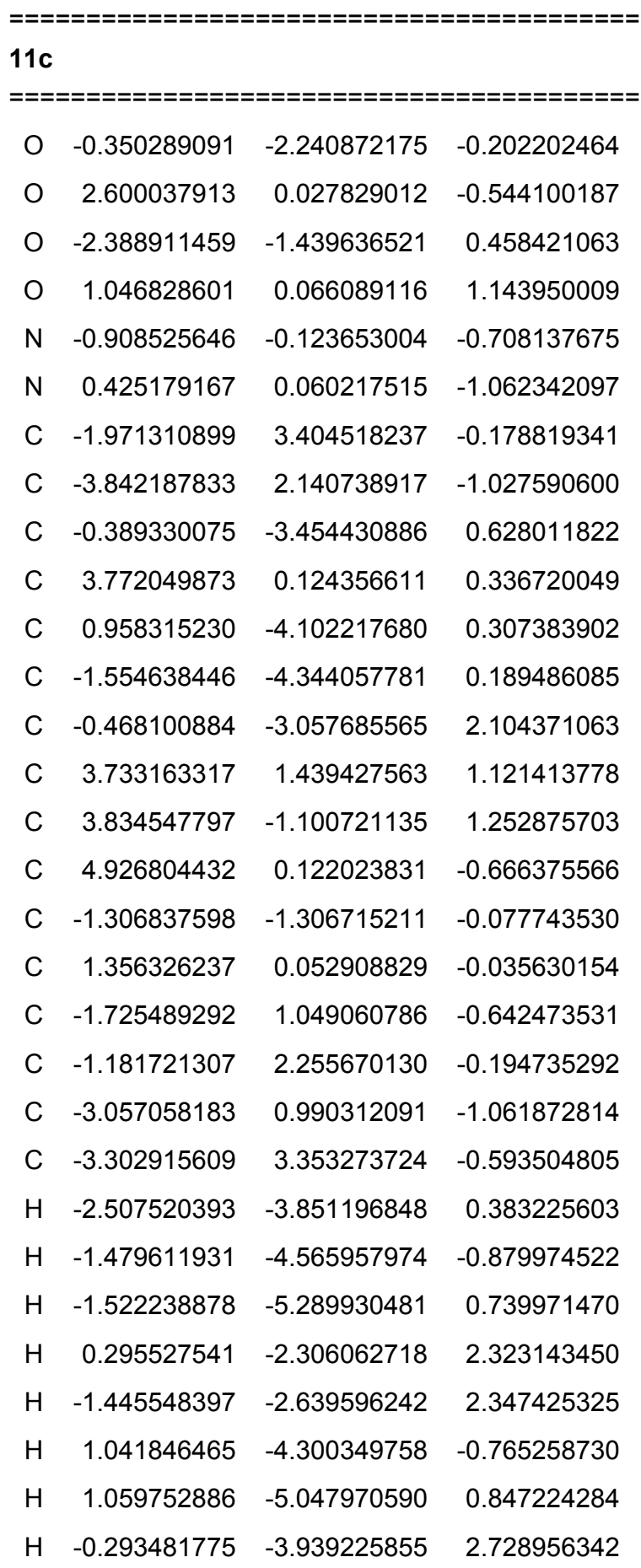

\begin{tabular}{|c|c|c|c|}
\hline-1 & 1.777046507 & 3.439693200 & 0.00 \\
\hline & 3.617666921 & 2.282825426 & 0.4000014 \\
\hline & 4.673578287 & 568817819 & 1.666406570 \\
\hline & 5.883973302 & 0.182202300 & 0.140054805 \\
\hline & 4.911856701 & -0.794044457 & -1.264035901 \\
\hline & 4.844424012 & 0.976259784 & -1344699160 \\
\hline & 18 & 78 & 1.824565120 \\
\hline & 3.811813247 & -2.017379016 & 0.655 \\
\hline & 2.907356067 & 1.446338425 & 1.83296506 \\
\hline & 2.993586749 & -1.106740663 & 1.94 \\
\hline & 979 & 6973 & 475 \\
\hline & -3.468596246 & 0.045570152 & -1.395930822 \\
\hline & 59585 & 5986 & -0.4 \\
\hline & -1.54 & 4.3 & 0 \\
\hline & -4.877826128 & 2.089620006 & -1.00 \\
\hline & 1.48 & .899353549 & -5.5 \\
\hline & 0.271581810 & -1.211266542 & -4.650881080 \\
\hline & 2.40616 & 0.209349812 & 76 \\
\hline & 2.081350502 & -1.810 & -5.6 \\
\hline & 1.119154601 & 89991 & \\
\hline & 0.574271127 & -1.3 & 36 \\
\hline & -0.177222695 & -2.154331836 & -4.98446090 \\
\hline & -0.498202217 & -0.443146592 & -4.793505238 \\
\hline & 1.662153299 & 1.421558693 & -4.410041101 \\
\hline & 0.000 & 2 & -0.4 \\
\hline & 0.0010 & -0.191 & -7.2200 \\
\hline & $a$ & 5 & -2.468842211 \\
\hline & 379 & 998 & 2 \\
\hline 1 & 3279 & 98 & $\$ 18$ \\
\hline C & 1.125921610 & 1.200605843 & -3.018623524 \\
\hline $\mathrm{H}$ & 0.853434198 & 1.737880771 & -5.089652 \\
\hline & 2.34966 & 2.2750 & -4.36 \\
\hline & 1.112624670 & 2.070776011 & -2.36640967 \\
\hline
\end{tabular}

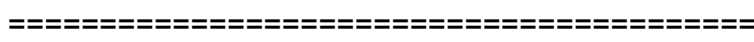
$11 \mathrm{~d}$

$\begin{array}{crrr}==================================== \\ \mathrm{O} & -0.399449861 & -2.024577641 & -0.852086947 \\ \mathrm{O} & 2.325856513 & 0.871742078 & -0.574502388 \\ \mathrm{O} & -2.378104865 & -1.653955860 & 0.219096722 \\ \mathrm{O} & 0.958380006 & 0.007723250 & 1.042870325 \\ \mathrm{~N} & -1.090569073 & 0.092278324 & -0.589179793\end{array}$




\begin{tabular}{|c|c|c|c|}
\hline & 70843 & 8 & \\
\hline & 129829 & 3 & \\
\hline & .762639913 & 194264172 & .00 \\
\hline & 0.271706653 & 3.432574907 & 6. \\
\hline & 2 & 1 & 0 \\
\hline & 1902 & 2 & -1.08531235 \\
\hline & 8 & 0 & -1.0 \\
\hline & 6. & 89845 & \\
\hline & 3376 & 58 & \\
\hline & 3.8876 & 79 & \\
\hline & & & \\
\hline & & & \\
\hline & ז & & \\
\hline & 9364 & & \\
\hline & 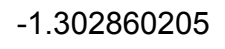 & & \\
\hline & -3.0 & & \\
\hline & 3.2 & & \\
\hline & 9875 & & \\
\hline & & & \\
\hline & - & -5 & -0 \\
\hline & 0.5 & & \\
\hline & $-1.12 \mathrm{~s}+3 \mathrm{~s}$ & & \\
\hline & 1.06 & -3 & \\
\hline & 1.275554666 & 3 & -0.88 \\
\hline & 0122519610 & 6 & \\
\hline & & & \\
\hline & & & \\
\hline & & & \\
\hline & 58 & & \\
\hline & 4.1812 & 0.82 & \\
\hline & $4 . \angle y$ & 4 & \\
\hline & 4.0 & 5 & \\
\hline & & & \\
\hline & & & \\
\hline & 799 & & \\
\hline & & & \\
\hline & - & 0 & \\
\hline & -3.70 & & \\
\hline & & & \\
\hline & -4.1 & & \\
\hline & & & \\
\hline & טעם זדידלט. & 2.491505032 & -4.8993402 \\
\hline
\end{tabular}
C -0.060481206
2.125966632
$-5.402182472$
H 1.696101104
3.347873191
$-5.487947659$
H 1.276888908
2.845255451
$-3.863603757$
C 2.682144382
0.622904426
$-3.647639029$
H 3.345175154
1.775966046
$-5.312401948$
H 2.105516836
0.625619384
$-5.747346342$
C -0.651118047
0.845264661
$-4.770272465$
H $\quad-0.035407368$
1.989282428
$-6.491714750$
H $\quad-0.735206254$
2.970588642
$-5.211062347$
C $\quad 1.585443075$
$-0.301211521$
$-3.074482957$
H 2.937085100
1.36688612
$-2.889103942$
H 3.579415476
0.00555811
$-3.790923355$
C $\quad-0.641606777$
0.868102096
$-3.268104430$
H -0.092303222
$-0.020868094$
$-5.142507375$
H $\quad-1.680463558$
0.719561072
$-5.127128329$
C $\quad 0.352256207$
0.379595150
$-2.510702732$
H 2.023994073
$-0.928251525$
$-2.295827972$
H 1.256543502
$-0.990581269$
$-3.861791801$
H -1.49403476
1.331654635
$-2.780091482$

\section{$11 \mathrm{e}$}

\begin{tabular}{|c|c|c|c|}
\hline $\mathrm{O}$ & -0.463788783 & -2.192873554 & -0.441221705 \\
\hline $\mathrm{O}$ & 2.483885766 & 0.283556968 & -0.836654259 \\
\hline $\mathrm{O}$ & -2.371359880 & -1.438819058 & 0.563353625 \\
\hline $\mathrm{O}$ & 1.055665714 & 0.070735501 & 0.937003072 \\
\hline $\mathrm{N}$ & -1.016316665 & -0.036279633 & -0.679578742 \\
\hline $\mathrm{N}$ & 0.267628336 & 0.078701193 & -1.216405729 \\
\hline C & -1.740561656 & 3.592480584 & -0.277354195 \\
\hline C & -3.805082895 & 2.376161991 & -0.099340595 \\
\hline$C$ & -0.495008592 & -3.530327589 & 0.164808322 \\
\hline C & 3.697387449 & 0.387659727 & -0.009162509 \\
\hline C & 0.749505654 & -4.182512063 & -0.439408993 \\
\hline C & -1.765991382 & -4.267553262 & -0.265004627 \\
\hline$C$ & -0.363786679 & -3.401802082 & 1.684861386 \\
\hline$C$ & 3.620948778 & 1.637411229 & 0.872630682 \\
\hline C & 3.882779718 & -0.893977130 & 0.809366076 \\
\hline C & 4.795905809 & 0.525982272 & -1.064113474 \\
\hline C & -1.370434731 & -1.262681139 & -0.102434650 \\
\hline C & 1.285316202 & 0.121983655 & -0.257268349 \\
\hline C & -1.732510859 & 1.175899306 & -0.440268630 \\
\hline
\end{tabular}




\begin{tabular}{|c|c|c|c|}
\hline & 1.045242171 & 2.3968 & .44 \\
\hline & 3.125893861 & 373 & -0.275096014 \\
\hline & 3.123140324 & 592899743 & .096 \\
\hline & -2.652287586 & 0396946 & 11 \\
\hline & -1.8 & 32 & -1.357072722 \\
\hline & -1.7298 & -5.3 & 0.0950 \\
\hline & 0.480509415 & 6558 & \\
\hline & -1.267140868 & -2. & \\
\hline & 0.6825 & & 1.0 \\
\hline & 083 & -5.2 & \\
\hline & -0.18 & & \\
\hline & 1.6 & -3.6 & -0. \\
\hline & 3.4 & & \\
\hline & 4.0 & & \\
\hline & 5.7 & & \\
\hline & 4.810 & 83 & \\
\hline & 4.624 & & \\
\hline & 4.85 & & \\
\hline & 3.861 & & \\
\hline & & & \\
\hline & 3.05 & & \\
\hline & 0.026 & & \\
\hline & -0.0 & & -0 \\
\hline & ; & & \\
\hline & . & & \\
\hline & -4 & 4 & \\
\hline & 1.2 & & \\
\hline & 0.4 & -0.2 & \\
\hline & -0.46684 & & \\
\hline & 1.717832595 & $-1 . \varepsilon$ & \\
\hline & 2 & -1 & \\
\hline & -0.00511 & & \\
\hline & -0 & & \\
\hline & -1 . & -0. & \\
\hline & 2.5 & -2 & -4 \\
\hline & 1.6 & $-0 . \subseteq$ & -5 \\
\hline & 0.56 & -2.8 & $-4 . \varsigma$ \\
\hline & -2.3 & & \\
\hline & -2.503780045 & & \\
\hline & -2.140 & -1.5 & -3.5 \\
\hline & 0.2 & -3.5 & -4 . \\
\hline & 1.136363000 & -3.396833451 & $-0.000<0<0$ \\
\hline
\end{tabular}
C $\quad-0.672533418$
$-2.214747576$
$-5.664756898$
H $\quad-0.799744048 \quad-2.748410608 \quad-6.614875471$
H $\quad-0.476278747 \quad-1.173053869 \quad-5.947616660$
C $\quad-2.014745138-2.305197812 \quad-4.919589616$
H $\quad-2.800891709-1.946199840 \quad-5.598565866$
H $\quad-2.236867764-3.366133121 \quad-4.736432672$
H $\quad-1.452262922 \quad-1.980194435 \quad-2.853309404$
$\begin{array}{llll}H & -3.148358542 & -1.742536369 & -3.197650797\end{array}$

\begin{tabular}{|c|c|c|c|}
\hline \\
\hline d & -2.370794351 & 2.242364881 & $-1.779366 c$ \\
\hline c & -3.515898800 & 2.815306512 & 1.4257536 \\
\hline D & -1.8895 & 4086 & 036 \\
\hline 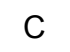 & -0.99 & 096 & 1.1279348 \\
\hline C & -0.72548 & 45341 & 3323 \\
\hline C & -3.411496090 & 2.632765182 & 2.95 \\
\hline C & -4.042773597 & 4.236 & 1.1 \\
\hline 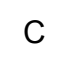 & -4.602187836 & 1.856016481 & 0.8 \\
\hline c & -2.037604569 & -0.16 & 1.245 \\
\hline 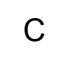 & 0.0368 & 0 & 0.0 \\
\hline 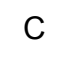 & -0.2979 & 1.1 & 2.4895014 \\
\hline C & -0.641 & 7 & 2.1792690 \\
\hline 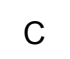 & 6 & 3.7 & 0.2404234 \\
\hline r & -1.19 & 54 & \\
\hline 7 & -2.6 & & \\
\hline 7 & -4 & & \\
\hline & -3 & & \\
\hline 1 & & & \\
\hline - & -3.43 & & \\
\hline 1 & & & \\
\hline & -4.40 & & \\
\hline 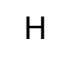 & -5.5 & & \\
\hline 1 & & & -1 \\
\hline 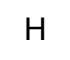 & -2.7 & -0.009582991 & \\
\hline 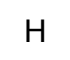 & -1.5 & 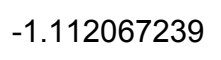 & \\
\hline 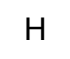 & -2.5 & -5 & \\
\hline - & 0.87 & 0 & $x_{1}+2$ \\
\hline & -0.4 & 6 & -0.8805 \\
\hline & 0. & 6 & \\
\hline & 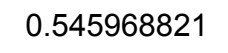 & 24425 & 36 \\
\hline
\end{tabular}




\begin{tabular}{|c|c|c|c|}
\hline & & & \\
\hline & 974986488 & 97861665 & $57-2>$ \\
\hline & 9 & 0 & 23777 \\
\hline & 91247 & 3 & 2.238 \\
\hline & 40 & 966 & 53 \\
\hline & 2 & 37 & 6037 \\
\hline & 12 & 2 & 66 \\
\hline & 1.3 & 4 & 30 \\
\hline & 2.153187566 & 66 & 0.109 \\
\hline & -0.4 & 4 & \\
\hline & 2 & & \\
\hline & 8 & & \\
\hline & 2.1 & & \\
\hline & -1.52 & & \\
\hline & -4.98 & & \\
\hline & & & \\
\hline & & & \\
\hline & & & \\
\hline & & & \\
\hline & -2.8543 & 1 & \\
\hline & -1.858003545 & & \\
\hline & -1.259 & & -4 \\
\hline & 0.5 & 8 & \\
\hline & -0.0 & -0.3 & \\
\hline & -0.11 & -2.4 & -3 \\
\hline & 1.4 & $-1 . \varepsilon$ & \\
\hline & -1.3 & -2.0 & -3 \\
\hline & -1.9 & -0.8 & \\
\hline & 2 & -2 & \\
\hline & -3 & 1 & \\
\hline & s & & \\
\hline & 1 & & \\
\hline & 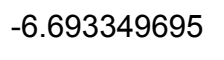 & & \\
\hline & & & \\
\hline & ( & & \\
\hline & 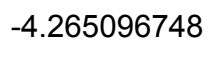 & & \\
\hline & & & \\
\hline & & & \\
\hline & & & \\
\hline & & & \\
\hline & & & enten \\
\hline & & & \\
\hline
\end{tabular}

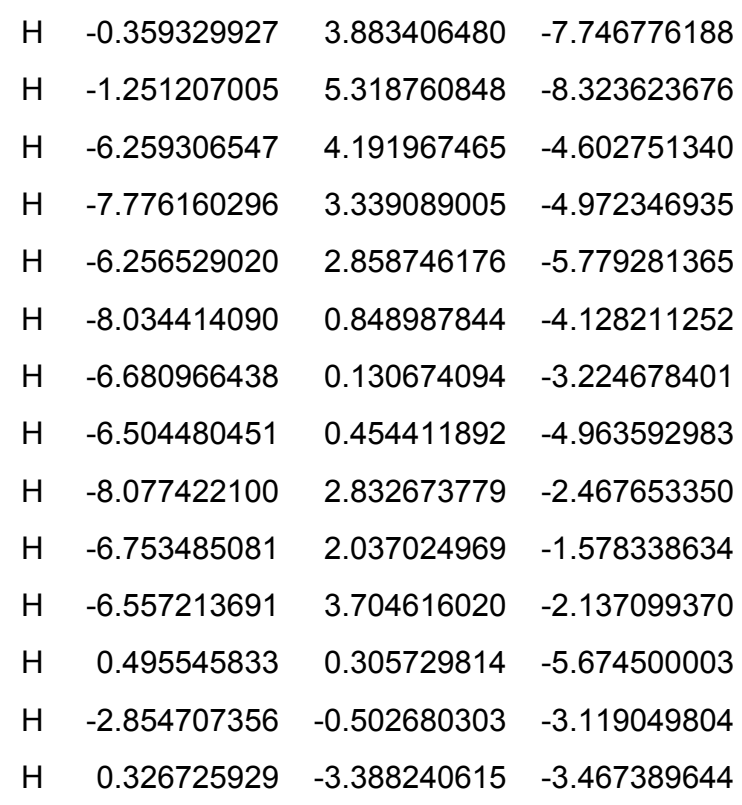

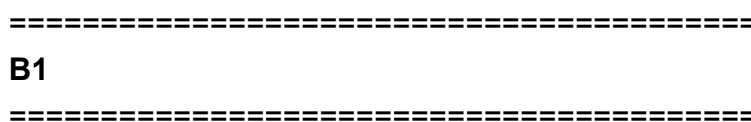

$\begin{array}{crrr}\text { Pd } & -2.428024010 & 2.665532181 & -1.670617995 \\ \text { C } & -4.204069504 & 3.531811120 & 1.454018664 \\ \text { P } & -2.451520857 & 3.258410176 & 0.663309374 \\ \text { C } & -1.586870450 & 1.796025592 & 1.620850446 \\ \text { C } & -1.402273896 & 4.864195535 & 1.028990280 \\ \text { C } & -4.286175291 & 3.468949503 & 2.991834905 \\ \text { C } & -4.747365730 & 4.901197286 & 0.991120467 \\ \text { C } & -5.160626628 & 2.480657803 & 0.843451539 \\ \text { C } & -2.592411899 & 0.635728020 & 1.755789262 \\ \text { C } & -0.449889161 & 1.251755160 & 0.718704195 \\ \text { C } & -1.020258622 & 2.114485991 & 3.017392476 \\ \text { C } & -1.475427488 & 5.437791829 & 2.457730814 \\ \text { C } & 0.071578069 & 4.549386536 & 0.686584020 \\ \text { C } & -1.832490856 & 5.982164206 & 0.049697369 \\ \text { H } & -3.633258962 & 4.194721697 & 3.481706706 \\ \text { H } & -5.316293573 & 3.688780923 & 3.307350796 \\ \text { H } & -4.039522087 & 2.477916415 & 3.377801205 \\ \text { H } & -4.633493138 & 5.031555041 & -0.089859068 \\ \text { H } & -4.271416197 & 5.742577578 & 1.500388558 \\ \text { H } & -5.820530417 & 4.950858107 & 1.220160282 \\ \text { H } & -4.888041031 & 1.451821480 & 1.061283099 \\ \text { H } & -6.169928096 & 2.652134553 & 1.246863008 \\ \text { H } & -5.202568432 & 2.566322572 & -0.243168626 \\ \text { H } & -3.364842684 & 0.822933960 & 2.504943132\end{array}$




\begin{tabular}{|c|c|c|c|}
\hline & 36 & 445 & \\
\hline & 3.077112547 & 0.421760239 & 806923 \\
\hline & 73 & 0 & 6982 \\
\hline & 838728844 & 0.924838281 & .25 \\
\hline & 66 & 0.380 & 1.2 \\
\hline & 94 & 2.82 & 30404 \\
\hline & 12 & 2 & 662 \\
\hline & 34 & 0 & 74 \\
\hline & 1.179802952 & 4.72 & 3.2 \\
\hline & 0.7 & 6.2 & 2 \\
\hline & 26 & 33 & \\
\hline & 8 & & \\
\hline & 0.5 & & \\
\hline & & & \\
\hline & -2.80 & & \\
\hline & & & \\
\hline & 1.0 & & \\
\hline & & & \\
\hline & & & \\
\hline & 0 & 20 & \\
\hline & 0.7 & 3.6 & -5 \\
\hline & 1.66 & 3.4 & \\
\hline & 1.90 & & \\
\hline & 1.30 & & \\
\hline & 2.04 & 0.4 & -3 \\
\hline & 3.12 & 1.7 & -5 \\
\hline & -2.4 & 1 & -3 \\
\hline & -3.3 & 9 & \\
\hline & -2.4 & & \\
\hline & -3.7 & 6 & \\
\hline & & & \\
\hline & -1.5008 & & \\
\hline & - $12+2$ & & \\
\hline & 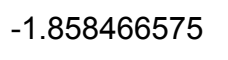 & & \\
\hline & 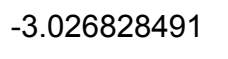 & & \\
\hline & (0) & & \\
\hline & & & \\
\hline & & & \\
\hline & & & \\
\hline & -3.779365880 & & \\
\hline & -6.675483321 & & (1) \\
\hline & & & 500 \\
\hline
\end{tabular}

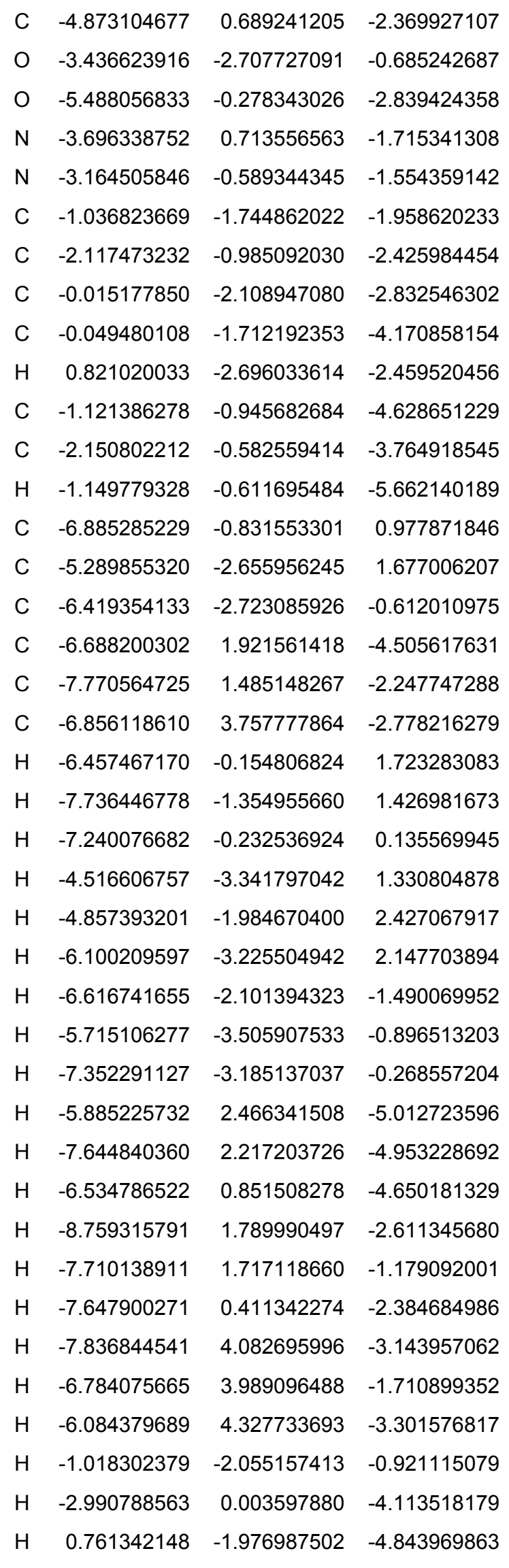

$\begin{array}{llll}\text { C } & -4.873104677 & 0.689241205 & -2.369927107\end{array}$

$\begin{array}{llll}\mathrm{O} & -5.488056833 & -0.278343026 & -2.839424358\end{array}$

$\begin{array}{llll}N & -3.696338752 & 0.713556563 & -1.715341308\end{array}$

C $\quad-1.036823669-1.744862022 \quad-1.958620233$

C $\quad-0.049480108 \quad-1.712192353 \quad-4.170858154$

H $\quad 0.821020033 \quad-2.696033614 \quad-2.459520456$

C $\quad-2.150802212 \quad-0.582559414 \quad-3.764918545$

C $\quad-6.885285229-0.831553301$

$\begin{array}{llll}\text { C } & -6.419354133 & -2.723085926 & -0.612010975\end{array}$

C $\quad-6.688200302 \quad 1.921561418-4.505617631$

$\begin{array}{llll}\text { C } & -7.770564725 & 1.485148267 & -2.247747288\end{array}$

$-6.856118610-3.757777864,-2.778216279$

$\begin{array}{llll}\text { H } & -7.736446778 & -1.354955660 & 1.426981673\end{array}$

$\begin{array}{llll}\text { H } & -7.240076682 & -0.232536924 & 0.135569945\end{array}$

$\begin{array}{llll}\text { H } & -4.516606757 & -3.341797042 & 1.330804878\end{array}$

$\begin{array}{llll}\text { H } & -4.857393201 & -1.984670400 & 2.427067917\end{array}$

$\begin{array}{llll}H & -6.100209597 & -3.225504942 & 2.147703894\end{array}$

H $\quad-6.616741655-2.101394323 \quad-1.490069952$

H $\quad-5.715106277 \quad-3.505907533 \quad-0.896513203$

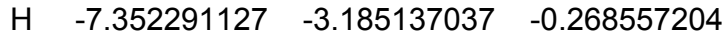

H $\quad-5.885225732 \quad 2.466341508 \quad-5.012723596$

H $\quad-7.644840360 \quad 2.217203726 \quad-4.953228692$

H $\quad-6.534786522 \quad 0.851508278 \quad-4.650181329$

H $\quad-8.759315791 \quad 1.789990497 \quad-2.611345680$

H $\quad-7.710138911 \quad 1.717118660 \quad-1.179092001$

H $\quad-7.647900271 \quad 0.411342274-2.384684986$

H $\quad-7.836844541 \quad 4.082695996 \quad-3.143957062$

H $\quad-6.784075665 \quad 3.989096488-1.710899352$

H $\quad-6.084379689 \quad 4.327733693 \quad-3.301576817$

H $\quad-1.018302379-2.055157413 \quad-0.921115079$

H $\quad 0.761342148 \quad-1.976987502 \quad-4.843969863$

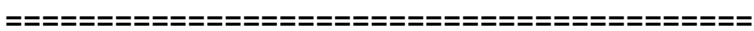




\section{B1-TS}

\begin{tabular}{|c|c|c|c|}
\hline$P d$ & -2.579407692 & 2.793061700 & -1.8 \\
\hline & 037771756 & 320912680 & 1.58 \\
\hline & 7 & 30 & \\
\hline & 272697612 & 1 & \\
\hline & -1.423590826 & 4.924262010 & 0.92 \\
\hline & -3.9415 & 3.140730382 & \\
\hline & $T .1$ & 4.663632148 & \\
\hline & -4.962211 & & \\
\hline & -2.151 & & \\
\hline & $-0.20 s$ & & \\
\hline & 0.58 & 2.1 & \\
\hline & -1.429428052 & 5.435832605 & 2.3 \\
\hline & 0.039 & 4.76 & 0.45 \\
\hline & $-20-2-3$ & 6.01 & \\
\hline & $-333>-$ & $3.8 \mathrm{~s}$ & \\
\hline & -4.9 & 3.23 & \\
\hline & -3.5 & 2.15 & 3.3 \\
\hline 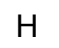 & $-4.7 \mathrm{~s}$ & 4.8 & \\
\hline 1 & -4.2 & 5.5 & \\
\hline-1 & -5.7 & 4.6 & \\
\hline $\mathrm{H}$ & $-4.5 \mathrm{~s}$ & 1.2 & 1.1 \\
\hline$\Pi$ & -5.9 & 2.3 & \\
\hline 1 & -5.10 & $r$ & 0.6 - \\
\hline $\mathrm{H}$ & -2.8 & & \\
\hline $\mathrm{H}$ & -1.5 & & \\
\hline & -2.7 & & \\
\hline & & & \\
\hline & s & & \\
\hline & & & \\
\hline & & & \\
\hline . & & & \\
\hline & 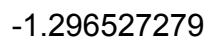 & & \\
\hline $\mathrm{H}$ & -1.004420393 & & \\
\hline $\mathrm{H}$ & -0.821475 & & \\
\hline $\mathrm{H}$ & -2 & & \\
\hline $\mathrm{H}$ & & & \\
\hline & & & \\
\hline & & & \\
\hline & & & \\
\hline & & & \\
\hline
\end{tabular}

\begin{tabular}{|c|c|c|c|}
\hline & 2 & 7847 & \\
\hline & .477311239 & 698144731 & $3638>$ \\
\hline & 1 & 66 & -3.617 \\
\hline & 87 & 008831 & -3.702 \\
\hline & 1.150037461 & 3.11 & -4.91 \\
\hline & 0.9 & 39 & -2 \\
\hline & 2.07 & 17 & -3 \\
\hline & 2.1 & 19 & -4 \\
\hline & $1.817 \varepsilon$ & 0.53 & -2.1 \\
\hline & 3.29 & 31 & \\
\hline & -2.3 & 5.1 & \\
\hline & -3.3 & & \\
\hline & $-2 . \varepsilon$ & & \\
\hline & -3.4 & & \\
\hline & & & \\
\hline & -1.7 & & \\
\hline & $6.35+3$ & & \\
\hline & & & \\
\hline & & & \\
\hline & & & \\
\hline & $-c$ & & \\
\hline & -4 & 52 & \\
\hline & -5.7 & 35 & \\
\hline & -3.7 & 33 & \\
\hline & -6.6 & & \\
\hline & -5.38 & & \\
\hline & -4.8 & 22 & \\
\hline & -3.33 & -2 & \\
\hline & -5.5 & 14 & \\
\hline & -3.6 & & \\
\hline & -3.1 & 91 & \\
\hline & $-0 . \varsigma$ & 88 & \\
\hline & -2 & & \\
\hline & 0.037580710 & 30 & \\
\hline & -0. & 9 & -4 \\
\hline & & -2.2 & -2 \\
\hline & $\cos ^{2}$ & 5 & - \\
\hline & $307-8$ & $0.75-8$ > & \\
\hline & & & \\
\hline & & & \\
\hline & -5.264199492 & -2.595069 & 1. \\
\hline & & & \\
\hline
\end{tabular}

$\begin{array}{llll}\text { H } & -2.049728602 & 5.693277847 & -1.032708191\end{array}$

$\begin{array}{llll}\mathrm{O} & -0.477311239 & 1.698144731 & -3.638121838\end{array}$

S $\quad 0.795069701 \quad 2.507805266 \quad-3.617399631$

$\begin{array}{llll}\text { C } & -2.176311787 & 3.008831975 & -3.702233703\end{array}$

$\begin{array}{llll}\text { O } & 1.150037461 & 3.113521003 & -4.912668323\end{array}$

$\begin{array}{llll}0 & 0.949325146 & 3.366029189 & -2.420396805\end{array}$

F $\quad 2.117866067 \quad 0.290846219 \quad-4.313160485$

F $\quad \begin{array}{llll}1.817865838 & 0.535793848 & -2.166372331\end{array}$

F $\quad 3.293811280 \quad 1.746405581 \quad-3.210469364$

$\begin{array}{llll}\text { C } & -2.395059947 & 5.152998770 & -4.627959792\end{array}$

C $\quad-3.359439830 \quad 4.164993448 \quad-5.348671658$

H $\quad-3.436668780 \quad 4.353295179 \quad-6.424905578$

$\begin{array}{llll}H & -4.360784445 & 4.258947435 & -4.918180557\end{array}$

C $\quad-1.789201533 \quad 4.310580551 \quad-3.514698977$

H $\quad-1.590207422 \quad 5.478034771 \quad-5.305086228$

$\begin{array}{llll}\text { H } & -3.574108800 & 1.966680774 & -5.012254113\end{array}$

$\begin{array}{llll}H & -2.024630579 & 2.447938172 & -5.737806605\end{array}$

$\begin{array}{lllll}\text { O } & -4.735231730 & -0.866122052 & -0.197533781\end{array}$

$\begin{array}{llll}\text { C } & -5.787946014 & -1.757224585 & 0.285088076\end{array}$

C $\quad-3.716101338-1.390892453 \quad-0.930888586$

$\begin{array}{llll}\text { O } & -5.383309267 & 2.120211288 & -2.523109181\end{array}$

$\begin{array}{llll}\text { C } & -4.882020053 & 0.831110822 & -2.511503971\end{array}$

$\begin{array}{llll}\mathrm{O} & -3.336837183 & -2.552675922 & -0.855295832\end{array}$

N $\quad-3.674512684 \quad 0.844157141 \quad-1.910550232$

$\begin{array}{llll}N & -3.133803357 & -0.446646291 & -1.744649649\end{array}$

C $\quad-0.930287034-1.407406308 \quad-2.208626429$

$\begin{array}{llll}\text { C }-2.135751322 & -0.877019635 & -2.669043019\end{array}$

$\begin{array}{llll}\text { C } & 0.037580710 & -1.816263680 & -3.122713712\end{array}$

H $\quad 0.986706011 \quad-2.203397271 \quad-2.762356064$

C $\quad-1.399077108 \quad-1.159103435 \quad-4.946508919$

C $\quad-2.374210142 \quad-0.755454752 \quad-4.038544910$

$\begin{array}{llll}\text { H } & -1.581603254 & -1.052937722 & -6.013227369\end{array}$

$\begin{array}{llll}\text { C } & -6.874475448 & -0.784511418 & 0.751460620 \\ \text { C } & -5.264199492 & -2.595069210 & 1.456789851\end{array}$

C $\quad-6.317170764 \quad-2.622877174 \quad-0.865438033$ 


\begin{abstract}
C $\quad-6.687911853 \quad 2.072223784 \quad-4.609318019$
$\begin{array}{llll}\text { C } & -7.791381061 & 1.705275402 & -2.355581098\end{array}$

$\begin{array}{llll}\text { C } & -6.775065429 & 3.939643064 & -2.910876037\end{array}$

$\begin{array}{llll}\mathrm{H} & -6.500724092 & -0.138383958 & 1.550168897\end{array}$

$\begin{array}{llll}\text { H } & -7.740274534 & -1.340220967 & 1.127914675\end{array}$

$\begin{array}{llll}\text { H } & -7.192553560 & -0.153069835 & -0.081852084\end{array}$

H $\quad-4.467274977 \quad-3.256327532 \quad 1.116075146$

$\begin{array}{llll}H & -4.868051392 & -1.938232704 & 2.238965882\end{array}$

$\begin{array}{llll}\text { H } & -6.077870532 & -3.192657416 & 1.884915160\end{array}$

H $\quad-6.524357476 \quad-1.982836905 \quad-1.727208437$

H $\quad-5.582512059-3.373070927 \quad-1.159751720$

$\begin{array}{llll}\text { H } & -7.237965339 & -3.127259577 & -0.550758045\end{array}$

$\begin{array}{llll}H & -5.867430686 & 2.576463687 & -5.127713301\end{array}$

H $\quad-7.632563565 \quad 2.400713233 \quad-5.058464193$

$\begin{array}{llll}H & -6.578910663 & 0.995671333 & -4.742471689\end{array}$

$\begin{array}{llll}H & -8.769512945 & 2.042882989 & -2.718463575\end{array}$

$\begin{array}{llll}H & -7.720223779 & 1.936503180 & -1.287480167\end{array}$

$\begin{array}{llll}\text { H } & -7.706145538 & 0.626760428 & -2.489844972\end{array}$

$\begin{array}{llll}\text { H } & -7.711988015 & 4.321544627 & -3.331262382\end{array}$

$\begin{array}{llll}\text { H } & -6.742325083 & 4.178484886 & -1.843041584\end{array}$

H $\quad-5.936163945 \quad 4.448820430 \quad-3.393491093$

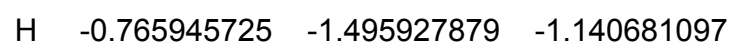

H $\quad-3.330317636 \quad-0.367142400 \quad-4.369552763$

H $\quad 0.579092735 \quad-1.982104237 \quad-5.202657989$
\end{abstract}

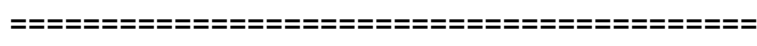

B5

$\begin{array}{crrr}==================================== \\ \text { Pd } & -2.518938719 & 2.722628161 & -1.625808121 \\ \text { C } & -4.057723291 & 3.576134315 & 1.489045929 \\ \text { P } & -2.317280822 & 3.222468607 & 0.697114762 \\ \text { C } & -1.521720697 & 1.702851548 & 1.619917159 \\ \text { C } & -1.168925344 & 4.748800300 & 1.096028156 \\ \text { C } & -4.158580043 & 3.476797436 & 3.023559260 \\ \text { C } & -4.515752076 & 4.986282627 & 1.057186663 \\ \text { C } & -5.070517369 & 2.598186543 & 0.841979055 \\ \text { C } & -2.586614409 & 0.597900404 & 1.740215111 \\ \text { C } & -0.430313250 & 1.125557203 & 0.683790882 \\ \text { C } & -0.926676015 & 1.953685497 & 3.018465465 \\ \text { C } & -1.237730678 & 5.305525170 & 2.531848993 \\ \text { C } & 0.289270057 & 4.353542758 & 0.769354077 \\ \text { C } & -1.497064241 & 5.900882938 & 0.119395689\end{array}$

\begin{tabular}{|c|c|c|c|}
\hline & & & \\
\hline & 178 & 7395 & 3.33820713 \\
\hline & 3.964254586 & 465170 & 852 \\
\hline & .405756973 & 1 & 242 \\
\hline & 7 & 5 & \\
\hline & 1 & 37 & 1081 \\
\hline & 4 & 0 & 99 \\
\hline & 0 & 5 & 37384 \\
\hline & -5.0 & 99 & -0 \\
\hline & -3.32 & 992 & 25 \\
\hline & -2.0 & 30 & \\
\hline & -3.1 & 0.2 & \\
\hline & 0.3 & & \\
\hline & -0.8 & 1 & \\
\hline & $-0 . c$ & & \\
\hline & -0.0 & & \\
\hline & -0.5 & & \\
\hline & -1.6 & & \\
\hline & & & \\
\hline & & & \\
\hline & & & \\
\hline & & & \\
\hline & & & \\
\hline & 0.91 & & \\
\hline & -2.50 & & \\
\hline & -0.7 & & \\
\hline & -1.3 & & -0 \\
\hline & -0.75 & 98 & $-3 . \varepsilon$ \\
\hline & 0.65 & 1 & -3 \\
\hline & 0.8 & & \\
\hline & 1.1 & & \\
\hline & 1.60 & 39 & \\
\hline & & & \\
\hline & 1.5 & & -3.2 \\
\hline & 2. & & -4. \\
\hline & -4.14 & -0.96 & 皮 \\
\hline & -5.7 & - & 0.61 \\
\hline & -3 & 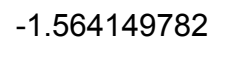 & 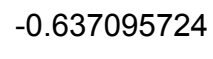 \\
\hline & -0 & 2.050579645 & -0.10 \\
\hline & & & \\
\hline & -4.815131892 & 0.551862 & 2.000000 \\
\hline & & & \\
\hline
\end{tabular}




\begin{tabular}{|c|c|c|c|}
\hline & 10 & & \\
\hline & 638079118 & 0.630221134 & -172097688 \\
\hline & 6 & 49 & -1. \\
\hline & 932566 & 71 & $.7 \varepsilon$ \\
\hline & 2.05 & -1.1 & -2. \\
\hline & 3 & -2 & -2 \\
\hline & 0.0 & $-1 . c$ & \\
\hline & 0. & -2 & -2.2 \\
\hline & 109799441 & -1.320 & \\
\hline & -2 & & \\
\hline & 8 & & \\
\hline & $0.4-5-3$ & & \\
\hline & -5.24 & & \\
\hline & 0.0 & & \\
\hline & -6.7 & & \\
\hline & & & \\
\hline & & & \\
\hline & & & \\
\hline & & & \\
\hline & -7.19 & & \\
\hline & 4.4 & & \\
\hline & 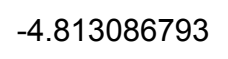 & -1 & \\
\hline & 6.0 & -3 & \\
\hline & -6.5 & -2.1 & \\
\hline & -5.6 & -3.5 & \\
\hline & -7.2 & -3.2 & \\
\hline & -5.9 & & \\
\hline & -7.6 & & \\
\hline & -6.5 & & \\
\hline & -8.7 & & \\
\hline & -7.59 & & \\
\hline & -7.59 & & \\
\hline & -7.75 & & \\
\hline & & & \\
\hline & 2 & & \\
\hline & -0.879964232 & & \\
\hline & & & \\
\hline & & & \\
\hline & & & \\
\hline & & & \\
\hline & & & \\
\hline & 0104 & .1070401 & \\
\hline
\end{tabular}

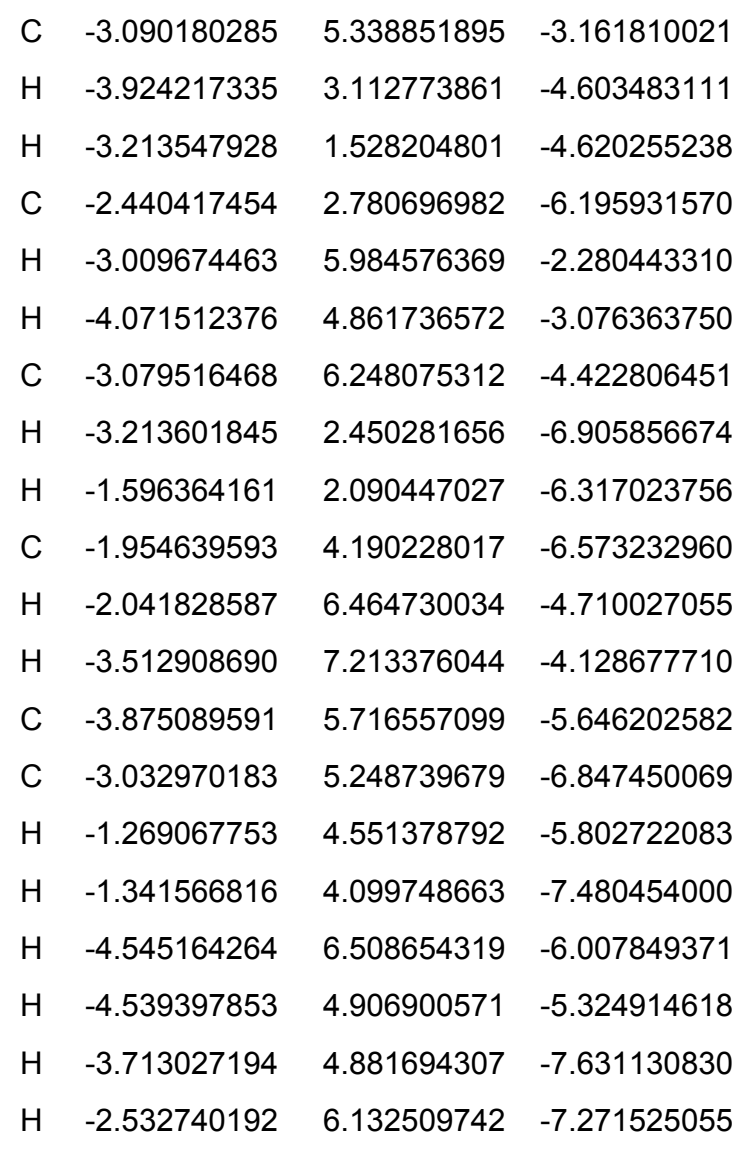

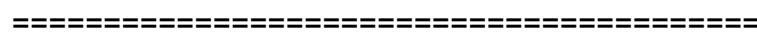

B5-TS

\begin{tabular}{cccc}
\hline \multicolumn{3}{c}{$=================================$} \\
$\mathrm{Pd}$ & -2.223638926 & 2.289413952 & -1.273772304 \\
C & -4.613583538 & 3.825168764 & 1.006476433 \\
P & -2.712903282 & 3.488297559 & 0.836389066 \\
C & -2.147187537 & 2.393106843 & 2.334627590 \\
C & -1.801259661 & 5.199469289 & 1.008930306 \\
C & -5.123132123 & 4.153220800 & 2.423876043 \\
C & -5.012154584 & 4.975097127 & 0.054524227 \\
C & -5.357745327 & 2.577327027 & 0.474673795 \\
C & -3.126564373 & 1.213170124 & 2.487557186 \\
C & -0.790820997 & 1.755871208 & 1.941266836 \\
C & -2.018894828 & 3.099878087 & 3.697696897 \\
C & -2.347413760 & 6.147053276 & 2.095621755 \\
C & -0.297352860 & 4.960198981 & 1.270705310 \\
C & -1.858727221 & 5.919508495 & -0.357794164 \\
H & -4.633356811 & 5.024776781 & 2.862470823 \\
H & -6.199951943 & 4.369035994 & 2.378138614 \\
H & -4.995629270 & 3.312250289 & 3.108569737 \\
H & -4.633511829 & 4.799987938 & -0.957787243
\end{tabular}




\begin{tabular}{|c|c|c|c|}
\hline & 55 & & \\
\hline & .108283458 & 016221506 & 005 \\
\hline & 5515 & 74 & 987414026 \\
\hline & 38 & 06387 & 58 \\
\hline & 3 & 99 & -0.5 \\
\hline & 0 & 94 & 8040 \\
\hline & 2.6 & 88 & 001 \\
\hline & -3.2 & 7 & 58 \\
\hline & 0.008583591 & 05 & 1.7 \\
\hline & -0.9 & & \\
\hline & -0.4 & & \\
\hline & -1.2 & & \\
\hline & 1. & & \\
\hline & 2. & & \\
\hline & 2. & & \\
\hline & & & \\
\hline & & & \\
\hline & & & \\
\hline & & & \\
\hline & & & \\
\hline & -2 & & \\
\hline & -1 & 6.8 & \\
\hline & -1.3 & & \\
\hline & & & \\
\hline & 2.58 & 10 & \\
\hline & 1.4 & & \\
\hline & 1.0 & 55 & \\
\hline & & & \\
\hline & & & \\
\hline & 1. & & \\
\hline & 2.9079736 & & \\
\hline & -5 & & \\
\hline & 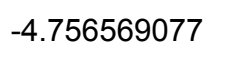 & & \\
\hline & -6.98 & & \\
\hline & $=0$ & & \\
\hline & & & \\
\hline & & & \\
\hline & & & \\
\hline & & & \\
\hline & & & \\
\hline & & & - \\
\hline & & & \\
\hline
\end{tabular}

\begin{tabular}{lrrr} 
C & -5.771326911 & 0.404904436 & -4.627202676 \\
C & -7.181609424 & 0.913913010 & -2.577213641 \\
C & -5.980889947 & 2.767884911 & -3.781683210 \\
O & -4.963993782 & -1.023853207 & -2.150617109 \\
N & -3.354356672 & 0.293562708 & -1.080614078 \\
C & -1.837299415 & -1.584026439 & -1.195390560 \\
C & -0.498303771 & -1.327215835 & -0.920146369 \\
C & -2.190212021 & -2.460652046 & -2.230420657 \\
C & 0.498811351 & -1.940454789 & -1.682131788 \\
C & 0.154071063 & -2.806420981 & -2.717980911 \\
H & 1.535279985 & -1.693708191 & -1.484227309 \\
H & -0.234811594 & -0.630673709 & -0.132769755 \\
C & -1.193061374 & -3.066744247 & -2.990312718 \\
H & 0.932133164 & -3.271752107 & -3.319030614 \\
H & -1.467064630 & -3.740545011 & -3.799374231 \\
H & -3.242923299 & -2.629561482 & -2.423519095 \\
H & -6.664684503 & 0.216298457 & 2.040839413 \\
H & -7.920570553 & -1.010827706 & 1.759027925 \\
H & -7.157402781 & -0.172689856 & 0.383475738 \\
H & -4.794319989 & -2.976686602 & 2.631967020 \\
H & -5.264215272 & -1.423405687 & 3.356975642 \\
H & -6.485368354 & -2.697668066 & 3.133755799 \\
H & -6.385744962 & -2.361388993 & -0.684615781 \\
H & -5.617118094 & -3.600811944 & 0.318464512 \\
H & -7.321730093 & -3.169876223 & 0.610703804 \\
H & -4.869566043 & 0.690252798 & -5.179591576 \\
H & -6.634480343 & 0.534556220 & -5.291278693 \\
H & -5.688371555 & -0.641566745 & -4.335187235 \\
H & -8.080788576 & 1.086611081 & -3.180496650 \\
H & -7.244464800 & 1.533131905 & -1.676543834 \\
H & -7.140367860 & -0.134466622 & -2.281634571 \\
H & -6.857290525 & 2.966537419 & -4.408275658 \\
H & -6.032080713 & 3.401409572 & -2.890715059 \\
H & -5.082519587 & 3.042054983 & -4.342794193 \\
\hline & -1.259820408 & 3.376268365 & -2.929598184 \\
\hline
\end{tabular}




$\begin{array}{lrrr}\text { H } & -2.163611198 & 5.286710105 & -3.149095174 \\ \text { H } & -3.132836152 & 3.993925975 & -3.811417868 \\ \mathrm{C} & -1.583111028 & 4.711293102 & -5.149984211 \\ \mathrm{H} & -1.255373659 & 0.239379161 & -6.031274037 \\ \mathrm{H} & -0.019761174 & 0.061400502 & -4.789008222 \\ \mathrm{C} & 0.018416099 & 1.977628702 & -5.751385633 \\ \mathrm{H} & -0.499229443 & 4.884592672 & -5.105365631 \\ \mathrm{H} & -2.028979235 & 5.675491732 & -5.430700315 \\ \mathrm{C} & -1.913350686 & 3.674549966 & -6.256611287 \\ \mathrm{C} & -0.722585859 & 2.848841534 & -6.777551933 \\ \mathrm{H} & 0.361529889 & 2.592300167 & -4.916821045 \\ \mathrm{H} & 0.932307835 & 1.604024122 & -6.232304224 \\ \mathrm{H} & -2.347944817 & 4.200646672 & -7.117575290 \\ \mathrm{H} & -2.704186915 & 3.002205770 & -5.902543901 \\ \mathrm{H} & -1.073358596 & 2.210261568 & -7.602110212 \\ \mathrm{H} & 0.000896314 & 3.548316918 & -7.223012374\end{array}$

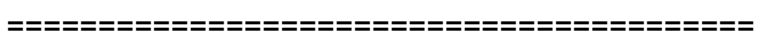

C1

\begin{tabular}{cccc}
\multicolumn{5}{c}{$=====================================$} \\
Pd & -1.864841124 & 1.670509357 & -1.309833629 \\
C & -3.390917108 & 3.664043982 & 1.432550279 \\
P & -2.058300215 & 2.372070686 & 0.919868167 \\
C & -2.425808446 & 0.688867876 & 1.807518151 \\
C & -0.306681047 & 2.997054669 & 1.450162937 \\
C & -3.729943032 & 3.656525172 & 2.937289493 \\
C & -2.924898803 & 5.081334852 & 1.029992685 \\
C & -4.675589609 & 3.393151980 & 0.614172849 \\
C & -3.925244772 & 0.349422558 & 1.662128719 \\
C & -1.669880886 & -0.423668176 & 1.036313277 \\
C & -2.036139874 & 0.627277010 & 3.295953119 \\
C & -0.238102327 & 3.595232039 & 2.868932100 \\
C & 0.711039820 & 1.836693950 & 1.347327336 \\
C & 0.167363366 & 4.050878966 & 0.418854085 \\
H & -2.856785860 & 3.833470684 & 3.568167823 \\
H & -4.448414079 & 4.460576453 & 3.140226752 \\
H & -4.197613890 & 2.722833209 & 3.256315144 \\
H & -2.664089022 & 5.129373656 & -0.030412322 \\
H & -2.084051193 & 5.439588539 & 1.626340874 \\
H & -3.757153115 & 5.775693532 & 1.200021604 \\
H & -5.126624936 & 2.422557195 & 0.814174485 \\
H & -5.418174227 & 4.157613627 & 0.875394832
\end{tabular}

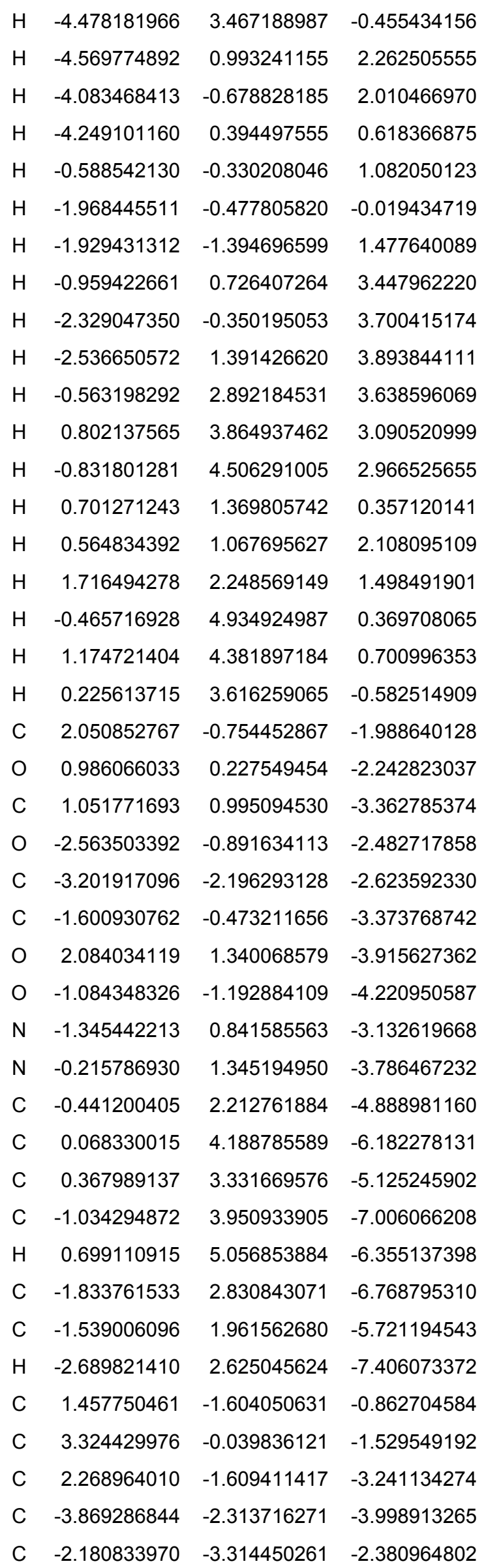




\begin{tabular}{|c|c|c|c|}
\hline & -42 & 87 & -1.513 \\
\hline & 1.288011664 & -0.996266150 & 0.030823549 \\
\hline & 2.144457055 & -2.414702453 & 600112669 \\
\hline & 0.504386494 & -2.037732950 & -1.177790212 \\
\hline & 3.715672650 & 0.588639741 & -2.329519270 \\
\hline & 3.111595132 & 0.588 & 2946 \\
\hline & 4.082522680 & -0.77 & 9530 \\
\hline & 1.305 & -1.97 & 55287 \\
\hline & 2.742058887 & -1.028910964 & -4.034284700 \\
\hline & 2.911904278 & -2.461151549 & -2.996245529 \\
\hline & -4.553036184 & -1.472406670 & -4.155285382 \\
\hline & -4.447783912 & -3.242073629 & -4.053030461 \\
\hline & -3.120991708 & -2.313 & 5825 \\
\hline & -2.683791550 & -4.2 & -2 \\
\hline & -1.699927182 & -3.1 & -1 \\
\hline & -1.415747970 & -3.30 & 81 \\
\hline & -4.8303 & -3 & 56 \\
\hline & -3 & -2 & \\
\hline & -4. & -1 & - \\
\hline & 2 & 0 & \\
\hline & 9 & 5 & \\
\hline & & & \\
\hline & -2 & 0 & -3. \\
\hline & $-4.196154 \mathrm{C}$ & & \\
\hline & -3.878 & 3. & -3. \\
\hline & -4.687 & & -4 \\
\hline & -4.8680 & 4.94 & 92 \\
\hline & -2.02 & 4.3 & -2.5 \\
\hline & -2.918 & 2 & 80 \\
\hline & -2.33 & 5.1 & -4.5 \\
\hline & -2.60 & 326 & -2.2 \\
\hline & -1.05913 & 4.7 & -2.1 \\
\hline & -4.6 & 2.56 & 138 \\
\hline & -3.6528208 & 2.339228329 & -3.916690965 \\
\hline
\end{tabular}

\section{C1-TS}

\begin{tabular}{|c|c|c|c|}
\hline $\mathrm{Pd}$ & -1.608095595 & 1.514932198 & $-1.07484127 c$ \\
\hline C & -3.359162625 & 3.453697681 & 1.366 \\
\hline & -1.892564744 & 2.2109 & 1.1413 \\
\hline & -2.213914019 & 0.668339184 & 2.2735 \\
\hline
\end{tabular}

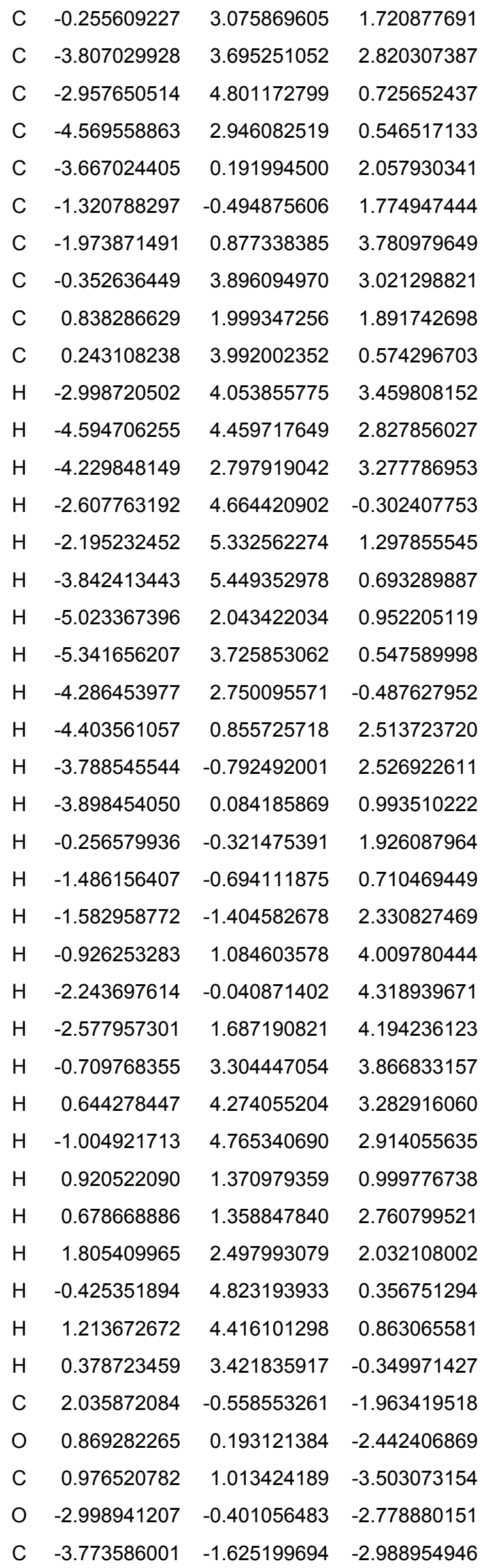




\begin{tabular}{|c|c|c|c|}
\hline & 94 & & \\
\hline & 006823072 & 1.297975771 & 088 \\
\hline & 382695 & 19797 & 3428 \\
\hline & 410299436 & 122094192 & -3.1 \\
\hline & 269542365 & 9003 & -3.8 \\
\hline & 30 & 2.3 & -5 \\
\hline & 0.23118 & 55 & 970 \\
\hline & 0.4 & 17 & -5 \\
\hline & 0.832049985 & 3.965 & -7.2 \\
\hline & 0.9 & 41 & \\
\hline & 32 & 29 & \\
\hline & -1.4 & & \\
\hline & 2.45 & & \\
\hline & & & \\
\hline & & & \\
\hline & & & \\
\hline & 1 & & \\
\hline & & & \\
\hline & & & \\
\hline & 0.9 & -0 & \\
\hline & 2.2 & -2 & \\
\hline & 0.6 & -2. & \\
\hline & 3.4 & & \\
\hline & 2.65 & & \\
\hline & 3.91 & -0.1 & \\
\hline & 1.75 & -2.0 & \\
\hline & 3.0 & -0.8 & \\
\hline & 8 & -2.1 & \\
\hline & 0 & -0.6 & \\
\hline & -5 & -2 & \\
\hline & 5 & & \\
\hline & -3 & -3 & \\
\hline & 2 & -2 & \\
\hline & & & \\
\hline & ? & -2 & \\
\hline & & & \\
\hline & & & \\
\hline & & & \\
\hline & & & \\
\hline & -0.989786950 & & \\
\hline & & & 1 \\
\hline & 204 & 580 & 50100 \\
\hline
\end{tabular}

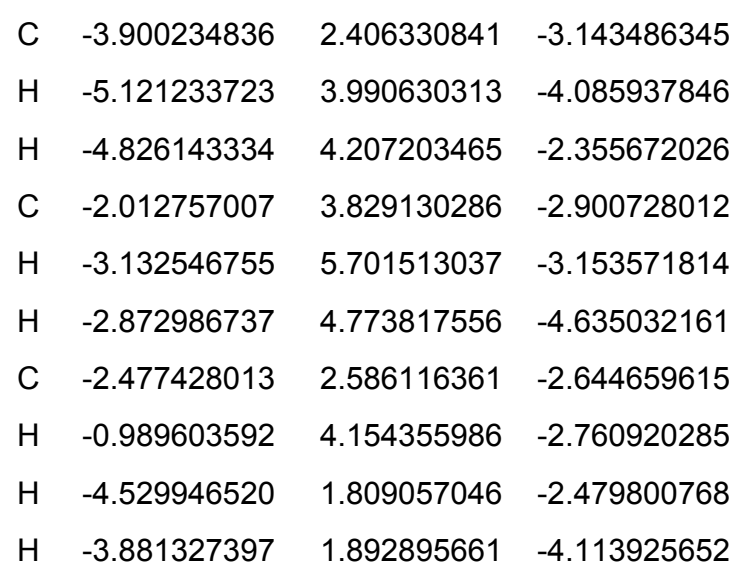

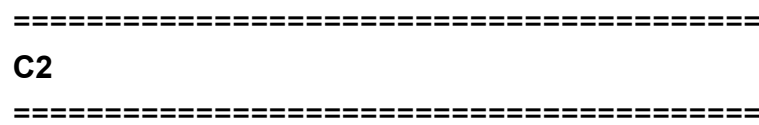

$\begin{array}{llll}\text { Pd } & -2.593644849 & 1.678483140 & -0.883355250\end{array}$

$\begin{array}{llll}\text { C } & -2.513283256 & 1.886956968 & 2.491614361\end{array}$

$\begin{array}{llll}\text { P } & -1.584973284 & 2.704780506 & 0.997601003\end{array}$

$\begin{array}{llll}\text { C } & 0.238434686 & 2.044944169 & 0.939326697\end{array}$

$\begin{array}{llll}\text { C } & -1.536353299 & 4.618238436 & 1.258326051\end{array}$

C $\quad \begin{array}{llll}-1.712912594 & 1.819493752 & 3.806444372\end{array}$

C $\quad-3.837439034 \quad 2.643802322 \quad 2.734481210$

$\begin{array}{llll}\text { C } & -2.922752089 & 0.448917549 & 2.080985946\end{array}$

$\begin{array}{llll}\text { C } & 0.228474101 & 0.504360383 & 1.070641778\end{array}$

$\begin{array}{llll}\text { C } & 0.812659983 & 2.337614116 & -0.469698882\end{array}$

$\begin{array}{llll}\text { C } & 1.182290470 & 2.620361319 & 2.012622387\end{array}$

$\begin{array}{llll}\text { C } & -1.221735682 & 5.055651339 & 2.703072274\end{array}$

$\begin{array}{llll}\text { C } & -0.495374108 & 5.251739240 & 0.308328032\end{array}$

$\begin{array}{llll}\text { C } & -2.898385613 & 5.210250133 & 0.830415397\end{array}$

$\begin{array}{llll}\text { H } & -1.383791939 & 2.800344969 & 4.153332872\end{array}$

$\begin{array}{llll}H & -2.351277212 & 1.392060871 & 4.590381046\end{array}$

$\begin{array}{llll}\text { H } & -0.835151379 & 1.174933127 & 3.722915058\end{array}$

$\begin{array}{llll}H & -4.420086464 & 2.737960482 & 1.812690062\end{array}$

H $\quad-3.692101820 \quad 3.636627500 \quad 3.163684015$

$\begin{array}{llll}H & -4.441030135 & 2.068807993 & 3.447394978\end{array}$

$\begin{array}{llll}\mathrm{H} & -2.081448738 & -0.207842567 & 1.873108405\end{array}$

$\begin{array}{llll}H & -3.492276689 & -0.001691717 & 2.903549673\end{array}$

$\begin{array}{llll}\text { H } & -3.574596786 & 0.442122619 & 1.200362425\end{array}$

$\begin{array}{llll}H & -0.041908734 & 0.162414602 & 2.071325763\end{array}$

$\begin{array}{llll}H & 1.240789207 & 0.133726647 & 0.867769779\end{array}$

$\begin{array}{llll}\text { H } & -0.435648014 & 0.030246860 & 0.341027892\end{array}$

H $\quad 0.925349722 \quad 3.398973236 \quad-0.684658176$

$\begin{array}{llll}H & 0.190079080 & 1.891844982 & -1.250249702\end{array}$ 


$\begin{array}{lrrr}\mathrm{H} & 1.811006496 & 1.886113671 & -0.537414089 \\ \mathrm{H} & 1.353260797 & 3.691420733 & 1.888461063 \\ \mathrm{H} & 2.159701838 & 2.128780412 & 1.924041832 \\ \mathrm{H} & 0.821736087 & 2.446322150 & 3.028431856 \\ \mathrm{H} & -0.277444658 & 4.651272832 & 3.072626884 \\ \mathrm{H} & -1.140473256 & 6.149878816 & 2.727687566 \\ \mathrm{H} & -2.011054262 & 4.778611441 & 3.404514886 \\ \mathrm{H} & -0.660762779 & 4.948287349 & -0.728102121 \\ \mathrm{H} & 0.534206855 & 5.024986727 & 0.589503140 \\ \mathrm{H} & -0.608355824 & 6.342057587 & 0.355462392 \\ \mathrm{H} & -3.744256068 & 4.827139771 & 1.398942417 \\ \mathrm{H} & -2.866992683 & 6.296483076 & 0.984857569 \\ \mathrm{H} & -3.076092322 & 5.026716197 & -0.227945334 \\ \mathrm{C} & -3.112808561 & -2.986562563 & -0.248821726 \\ \mathrm{O} & -3.422920488 & -1.614836806 & -0.670300877 \\ \mathrm{C} & -4.617021376 & -1.354556014 & -1.259059042 \\ \mathrm{O} & -1.243214101 & 0.239444692 & -3.211504252 \\ \mathrm{C} & -0.223293290 & -0.268548401 & -4.122559574 \\ \mathrm{C} & -2.479178572 & -0.345857095 & -3.163474966 \\ \mathrm{H} & -5.644660516 & -1.987198208 & -1.080402609 \\ \mathrm{H} & -1.404463425 & -2.186928851 & 0.830481175 \\ \mathrm{O} & -2.799575014 & -1.346416777 & -3.800379989 \\ \mathrm{~N} & -3.272292599 & 0.352656270 & -2.304140991 \\ \mathrm{~N} & -4.520008051 & -0.238864825 & -2.069785136 \\ \mathrm{C} & -5.637538592 & 0.268455371 & -2.787486260 \\ \mathrm{C} & -7.942855687 & 0.989259940 & -2.881027723 \\ \mathrm{C} & -6.891006180 & 0.404680954 & -2.176691345 \\ \mathrm{C} & -7.759089155 & 1.446145798 & -4.187657937 \\ \mathrm{H} & -8.911154571 & 1.095396765 & -2.398609815 \\ \mathrm{C} & -6.509648442 & 1.298845412 & -4.794245851 \\ \mathrm{C} & -5.451971217 & 0.711663447 & -4.103257283 \\ \mathrm{H} & -6.354781744 & 1.637981474 & -5.815421690 \\ - & -1.611181300 & -2.911591881 & 0.037090239 \\ \mathrm{C} & -3.902337269 & -3.332809780 & 1.016329302 \\ \mathrm{H} & -3.569668030 & -2.320545123\end{array}$

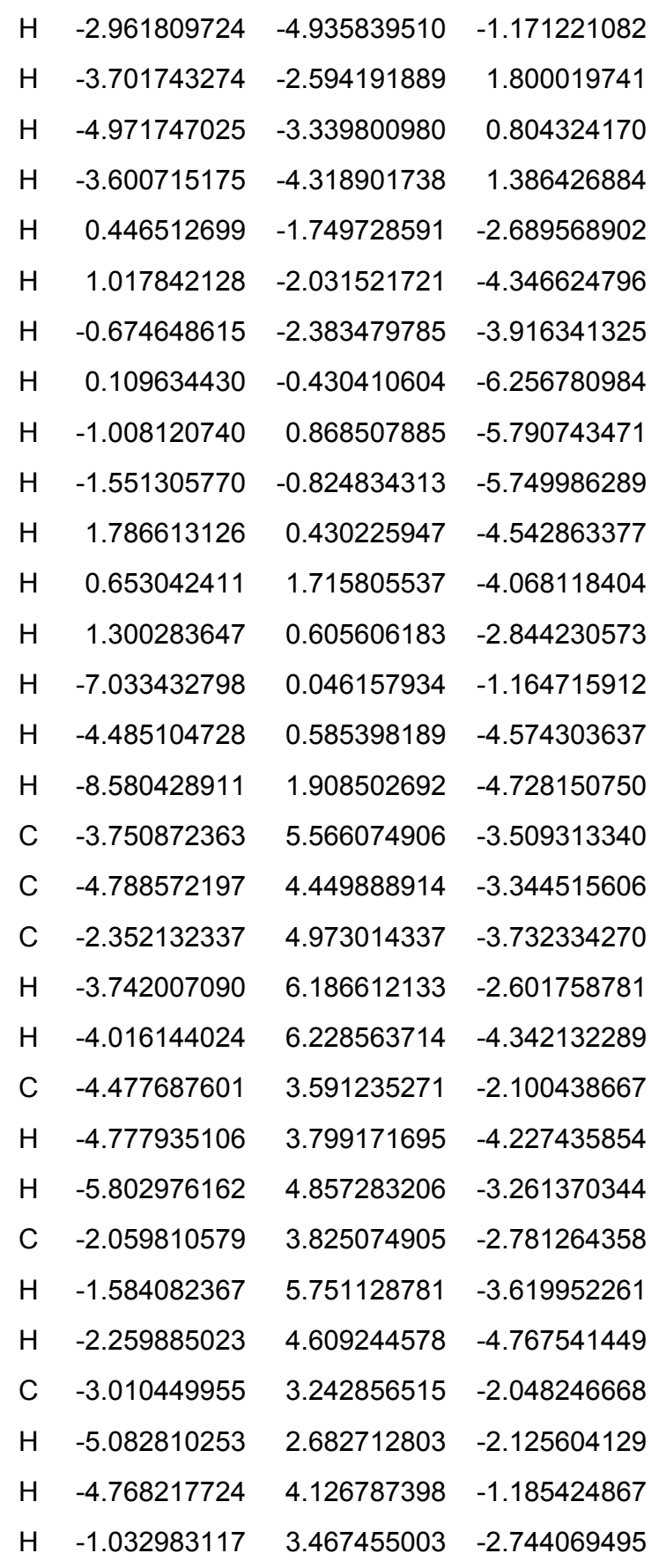

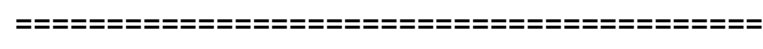

\section{C2-TS}

\begin{tabular}{|c|c|c|c|}
\hline $\mathrm{Pd}$ & -2.347095567 & 1.330216537 & -0.6 \\
\hline C & -2.289682054 & 2.254926419 & 2.7606 \\
\hline$P$ & -1.262890040 & 2.405823627 & 1.1219 \\
\hline C & 0.455358262 & 1.533670274 & 1.2 \\
\hline C & -0.926743201 & 4.284096237 & 0 \\
\hline C & -1.513330803 & 2.506371182 & 4.0677 \\
\hline C & -3.479018636 & 3.237538897 & \\
\hline
\end{tabular}




\begin{tabular}{|c|c|c|c|}
\hline & 2.921043892 & 5482 & \\
\hline & 214873285 & 148566689 & 03551777 \\
\hline & 1.079387834 & 01 & 1700 \\
\hline & 1.476547309 & 302743066 & 257266872 \\
\hline & 499729815 & 5.120450082 & 996 \\
\hline & 7066 & 69 & -0.3 \\
\hline & -2.195921140 & 58 & 0177 \\
\hline & -1.04 & 95 & 015 \\
\hline & -2.208050101 & 7589 & 4.9 \\
\hline & -0.73 & 1.75 & 68 \\
\hline & -4.02 & 20 & \\
\hline & 4 & 0 & \\
\hline & -4.1 & 7 & \\
\hline & 2.1 & & \\
\hline & -3.5 & & \\
\hline & -0.48 & & \\
\hline & 0.1 & & \\
\hline & & & \\
\hline & & & \\
\hline & $136>$ & & \\
\hline & 0.38 & 0.6 & -0. \\
\hline & 1.98 & 1 & \\
\hline & 1.79 & & \\
\hline & 2.37 & & \\
\hline & 1.09 & 2.53 & 3.2 \\
\hline & 0.38 & & \\
\hline & -0.26 & 45 & \\
\hline & -1.25 & 3 & \\
\hline & $-0.0 \varsigma$ & 9 & \\
\hline & 1.15 & & \\
\hline & & & \\
\hline & s & & \\
\hline & 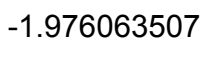 & 5.9620 & \\
\hline & 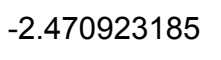 & 7 & \\
\hline & -3.475 & 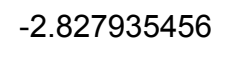 & \\
\hline & $x^{2}+2$ & $-\sigma^{2}+2$ & \\
\hline & 1 7 7 & & \\
\hline & & & \\
\hline & & & \\
\hline & -2.412660720 & -0.163397761 & -3.197948782 \\
\hline & -5.741175410 & -1.971983511 & -1.683383715 \\
\hline & & & \\
\hline
\end{tabular}

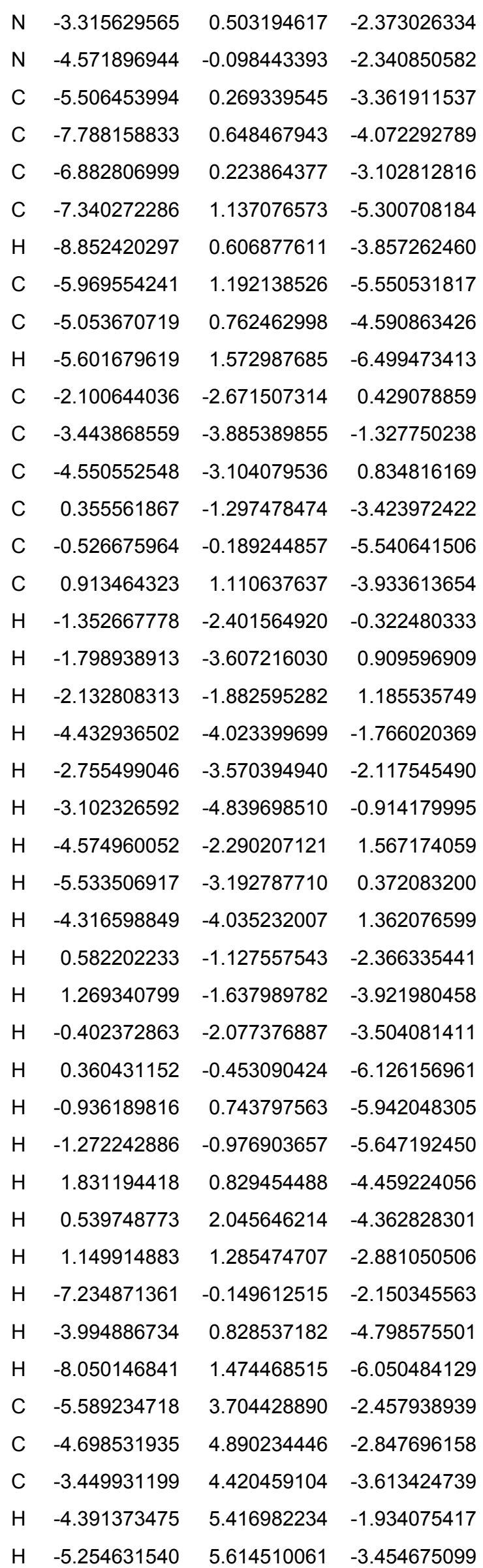




$\begin{array}{llll}\text { C } & -4.804153968 & 2.755523108 & -1.531713450 \\ \text { H } & -5.910667212 & 3.159961746 & -3.352689912 \\ \text { H } & -6.493770888 & 4.049753403 & -1.943152845 \\ \text { C } & -2.865434541 & 3.124367706 & -3.089449023 \\ \text { H } & -2.685358555 & 5.210393643 & -3.606550444 \\ \text { H } & -3.706932474 & 4.267332906 & -4.674619117 \\ \text { C } & -3.471679113 & 2.389345591 & -2.142188280 \\ \text { H } & -5.388259552 & 1.863278579 & -1.300866465 \\ \text { H } & -4.614597041 & 3.253819027 & -0.571651622 \\ \text { H } & -1.950699673 & 2.765757851 & -3.547188084\end{array}$

C3

\begin{tabular}{|c|c|c|c|}
\hline & 4 & 1 & -0.92 \\
\hline C & -0.626486143 & te & 1.685804124 \\
\hline & -1.375138346 & 11 & 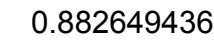 \\
\hline & 0.070489 & 15 & 0. \\
\hline C & -2.559912045 & 3.585642813 & 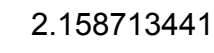 \\
\hline & 0.495731661 & 1.377519933 & 2.71268731 \\
\hline C & -1.757503430 & 0.336272138 & 2.36 \\
\hline C & -0.088130453 & 0.227409897 & 0.55 \\
\hline C & 1.14 & 13 & -03 \\
\hline 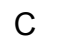 & 0.1 & 97 & 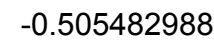 \\
\hline C & 6 & 8 & 1.67494060 \\
\hline C & -2.01 & 3 & 3.6 \\
\hline C & -2.872991368 & 5.023023218 & 1.6 \\
\hline C & -3.907233417 & 2.821326350 & 2.16 \\
\hline $\mathrm{H}$ & 0.185001434 & 2.024707315 & 3.53 \\
\hline $\mathrm{H}$ & 0.786155249 & 0.412595056 & 3.14 \\
\hline $\mathrm{H}$ & 1.391912266 & 1.804463151 & 2.25765606 \\
\hline $\mathrm{H}$ & -2.594651592 & 0.138793820 & 1.68971954 \\
\hline $\mathrm{H}$ & -2.140684222 & 0.820772681 & 3.26663948 \\
\hline $\mathrm{H}$ & -1.353978759 & -0.635672129 & 2.67566003 \\
\hline $\mathrm{H}$ & 0.722997824 & 0.670406642 & -0.022172525 \\
\hline $\mathrm{H}$ & 0.293771450 & -0.696307834 & 1.00425459 \\
\hline $\mathrm{H}$ & -0.867679096 & -0.070000394 & -0.16028993 \\
\hline $\mathrm{H}$ & 1.692497515 & 2.469063274 & 0.30327712 \\
\hline $\mathrm{H}$ & 1.875513684 & 3.883716045 & -0.72489006 \\
\hline $\mathrm{H}$ & 0.707150401 & 2.632628948 & -1.16846146 \\
\hline $\mathrm{H}$ & -1.120488228 & 5.767040368 & -0.03529588 \\
\hline $\mathrm{H}$ & -0.939868922 & 4.629679078 & -1.37324 \\
\hline
\end{tabular}

\begin{tabular}{lrrr}
$\mathrm{H}$ & 0.426475097 & 5.634113659 & -0.856149849 \\
$\mathrm{H}$ & 0.042024730 & 5.342780647 & 2.173406068 \\
$\mathrm{H}$ & 1.578126491 & 5.246465362 & 1.322813579 \\
$\mathrm{H}$ & 1.103465551 & 3.947516894 & 2.417152297 \\
$\mathrm{H}$ & -1.061957809 & 4.175615676 & 3.667556812 \\
$\mathrm{H}$ & -2.735590479 & 4.197089170 & 4.225705700 \\
$\mathrm{H}$ & -1.894588501 & 2.661697036 & 4.043679862 \\
$\mathrm{H}$ & -3.187970719 & 5.056438702 & 0.640921383 \\
$\mathrm{H}$ & -2.026567745 & 5.699590819 & 1.818790493 \\
$\mathrm{H}$ & -3.697409789 & 5.418066711 & 2.292793723 \\
$\mathrm{H}$ & -3.832465956 & 1.813284010 & 2.567492793 \\
$\mathrm{H}$ & -4.617342159 & 3.372131562 & 2.792938431 \\
$\mathrm{H}$ & -4.333630560 & 2.745159551 & 1.160737913 \\
$\mathrm{C}$ & -3.481637205 & -2.943299209 & -0.126903587 \\
$\mathrm{O}$ & -3.638070172 & -1.552572602 & -0.576693746 \\
$\mathrm{C}$ & -4.797255073 & -1.182085411 & -1.177084533 \\
$\mathrm{O}$ & -1.236243034 & 0.079976021 & -3.028412477 \\
$\mathrm{C}$ & -0.179980623 & -0.505415763 & -3.839287268 \\
$\mathrm{H}$ & -2.509447937 & -0.435021485 & -3.036130642 \\
$\mathrm{H}$ & -1.697740581 & -2.305721825 & 0.932323228 \\
$\mathrm{O}$ & -5.886003917 & -1.698889482 & -0.986250447 \\
$\mathrm{O}$ & -2.849620553 & -1.442268257 & -3.648272285 \\
$\mathrm{~N}$ & -3.285028433 & 0.355409658 & -2.246573131 \\
$\mathrm{~N}$ & -4.581663172 & -0.113885354 & -2.027653279 \\
$\mathrm{C}$ & -5.630564593 & 0.525268702 & -2.741100545 \\
$\mathrm{C}$ & -7.854101801 & 1.467286614 & -2.861121872 \\
$\mathrm{C}$ & -6.883379546 & 0.756179764 & -2.156617664 \\
$\mathrm{C}$ & -7.594048623 & 1.956203695 & -4.142734741 \\
$\mathrm{H}$ & -8.820077814 & 1.646585099 & -2.396438305 \\
$\mathrm{C}$ & -6.348080193 & 1.712879194 & -4.724682387 \\
$\mathrm{C}$ & -5.369288211 & 1.003926513 & -4.031938959 \\
$\mathrm{H}$ & -6.132655631 & 2.075692461 & -5.726294848 \\
$\mathrm{C}$ & -1.981753546 & -3.028692864 & 0.162953560 \\
$\mathrm{C}$ & -3.360659008 & -3.897461877 & -1.264623502 \\
\hline
\end{tabular}




$\begin{array}{llll}\text { H } & -3.542980387 & -4.914407684 & -1.011045286 \\ \text { H } & -4.023892610 & -2.448846148 & 1.912690849 \\ \text { H } & -5.369382408 & -3.067397926 & 0.928590442 \\ \text { H } & -4.115190217 & -4.181154218 & 1.531283660 \\ \text { H } & 0.328345882 & -1.945981401 & -2.303038783 \\ \text { H } & 0.982985330 & -2.335082516 & -3.907189423 \\ \text { H } & -0.748484231 & -2.581996044 & -3.567333589 \\ \text { H } & 0.293739045 & -0.780391872 & -5.936386837 \\ \text { H } & -0.794328815 & 0.584685356 & -5.607562683 \\ \text { H } & -1.414264714 & -1.080039805 & -5.529330640 \\ \text { H } & 1.897393639 & 0.080989633 & -4.094733029 \\ \text { H } & 0.782368115 & 1.440446600 & -3.828611217 \\ \text { H } & 1.243870422 & 0.394893097 & -2.470838192 \\ \text { H } & -7.090294115 & 0.371618085 & -1.166294149 \\ \text { H } & -4.400695424 & 0.819830690 & -4.478924095 \\ \text { H } & -8.353379559 & 2.514263991 & -4.683220605 \\ \text { C } & -4.575504761 & 5.304437744 & -2.073988666 \\ \text { C } & -4.884252376 & 5.581642324 & -3.554990103 \\ \text { C } & -4.070227118 & 4.735210527 & -4.549010465 \\ \text { H } & -5.950506426 & 5.398381793 & -3.740991617 \\ \text { H } & -4.713627655 & 6.649114922 & -3.750793040 \\ \text { C } & -4.442158859 & 3.806753034 & -1.717447118 \\ \text { H } & -5.367594914 & 5.748562264 & -1.457761478 \\ \text { H } & -3.643939090 & 5.809145802 & -1.786932482 \\ \text { C } & -2.581692096 & 4.578249609 & -4.195437569 \\ \text { H } & -4.163403939 & 5.181235083 & -5.547466367 \\ \text { H } & -4.507481806 & 3.733384619 & -4.614449754 \\ \text { C } & -3.093068427 & 3.234778856 & -2.079433832 \\ \text { H } & -5.227317151 & 3.216539827 & -2.206587195 \\ \text { H } & -4.612618729 & 3.687392281 & -0.643885376 \\ \text { C } & -2.297505535 & 3.553360783 & -3.103277620 \\ \text { H } & -2.149136742 & 5.556057865 & -3.928166038 \\ \text { H } & -2.036696240 & 4.253006753 & -5.090621762 \\ \text { H } & -1.362356604 & 3.007183357 & -3.218003354\end{array}$

\section{C3-TS}

\begin{tabular}{|c|c|c|c|}
\hline $\mathrm{Pd}$ & -2.425548325 & 1.447499825 & -0.73 \\
\hline C & -2.625342354 & 2.903214005 & 2.529041006 \\
\hline $\mathrm{P}$ & -1.398018867 & 2.473439388 & $1.089242 \mathrm{c}$ \\
\hline & -0.107211567 & 1.180472852 & 1.75 \\
\hline
\end{tabular}

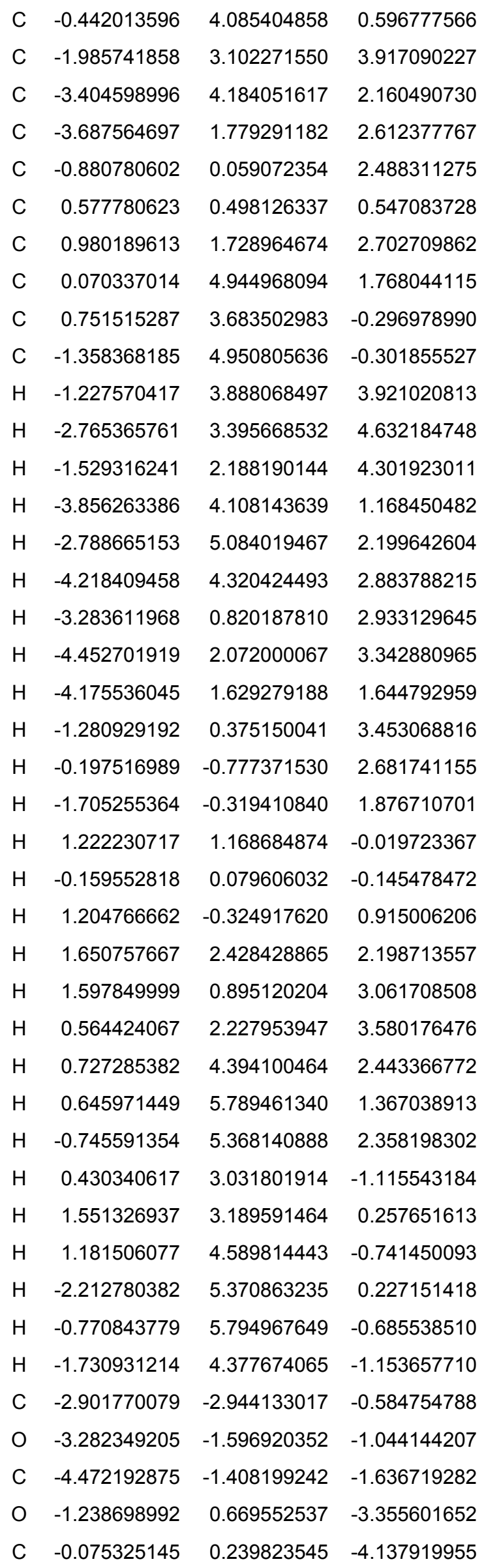




\begin{tabular}{|c|c|c|c|}
\hline & & & \\
\hline & 5.430029651 & 2.155001038 & 558 \\
\hline & 7 & 76 & 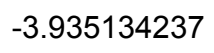 \\
\hline & 2915 & 6307 & -2 \\
\hline & 34 & 265 & -2 \\
\hline & 5.5 & 65 & \\
\hline & -7.82 & 01 & - \\
\hline & -6.8 & 78 & -3 \\
\hline & -7.491 & 836 & -5 \\
\hline & -8.8 & -0 & \\
\hline & -6.1 & & \\
\hline & -5.1 & & \\
\hline & 0.0 & & \\
\hline & -1.4 & & \\
\hline & 3. & & \\
\hline & & & \\
\hline & & & \\
\hline & & & \\
\hline & & & \\
\hline & -0.87 & -2 & \\
\hline & $-0 . \subseteq$ & -3 & \\
\hline & 1 & -2 & \\
\hline & -4.1 & -4 & \\
\hline & -2.6 & -3.5 & \\
\hline & -2.60 & $-4 . \varepsilon$ & \\
\hline & -3.5 & -2 & \\
\hline & -4.7 & -3.3 & \\
\hline & -3.3 & & \\
\hline & & -1 & \\
\hline & 1.38 & -1.3 & \\
\hline & -0.2 & & \\
\hline & & & \\
\hline & -0 & & \\
\hline & -1.1 & -0. & \\
\hline & & & \\
\hline & & & \\
\hline & & & \\
\hline & & & \\
\hline & & & \\
\hline & & & \\
\hline & & & (1) \\
\hline & & 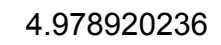 & \\
\hline
\end{tabular}

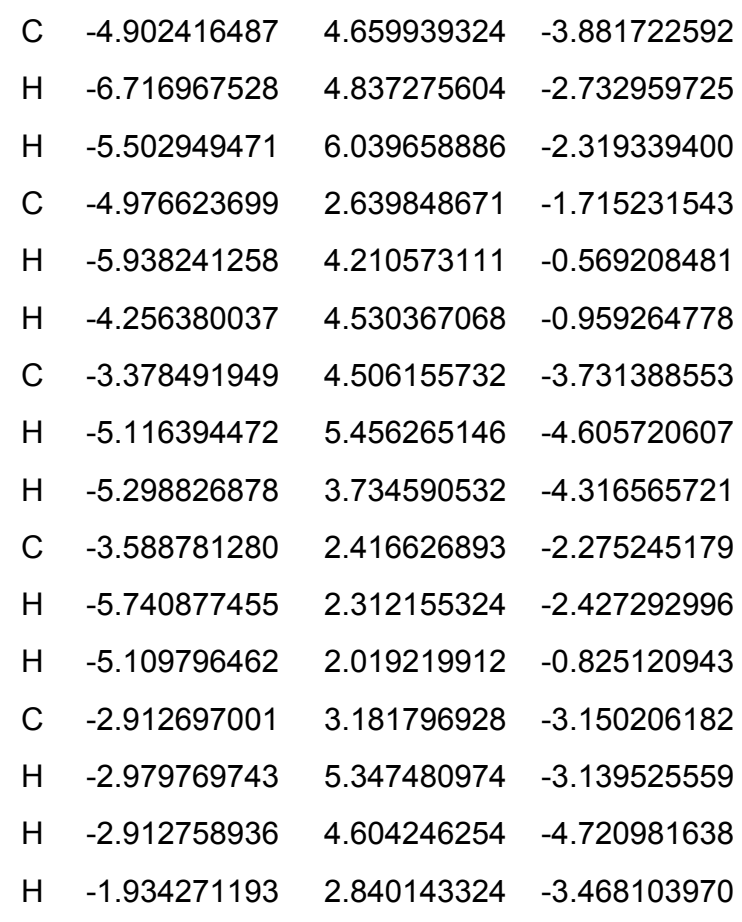

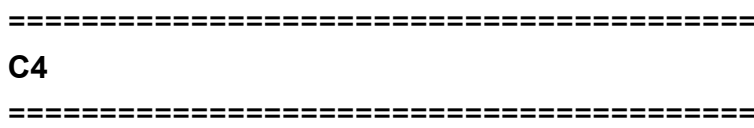

$\begin{array}{crrr}\text { Pd } & -2.614414200 & 1.786224100 & -0.982405650 \\ \text { C } & -2.854362000 & 1.948341500 & 2.344330200 \\ \text { P } & -1.712435000 & 2.727958800 & 0.982863730 \\ \text { C } & 0.062023313 & 1.963535600 & 1.137226300 \\ \text { C } & -1.595853700 & 4.632757000 & 1.267689900 \\ \text { C } & -2.220797900 & 1.819791500 & 3.742966700 \\ \text { C } & -4.153777400 & 2.776891800 & 2.453886000 \\ \text { C } & -3.302538100 & 0.546968350 & 1.855263100 \\ \text { C } & -0.053647700 & 0.427549150 & 1.254271800 \\ \text { C } & 0.823414000 & 2.214896700 & -0.188907570 \\ \text { C } & 0.897384800 & 2.487889800 & 2.321461400 \\ \text { C } & -1.403784600 & 5.042634600 & 2.742329800 \\ \text { C } & -0.432813220 & 5.218960000 & 0.434519550 \\ \text { C } & -2.882567500 & 5.293841700 & 0.719121800 \\ \text { H } & -1.882953900 & 2.777228100 & 4.143306300 \\ \text { H } & -2.971822700 & 1.420694500 & 4.436958000 \\ \text { H } & -1.376011100 & 1.127902000 & 3.755365300 \\ \text { H } & -4.625394400 & 2.914758300 & 1.475739700 \\ \text { H } & -4.002923000 & 3.754467100 & 2.914019200 \\ \text { H } & -4.863266600 & 2.228408600 & 3.085949100 \\ \text { H } & -2.489884900 & -0.154276860 & 1.683999800 \\ \text { H } & -3.961829000 & 0.103590620 & 2.612294000\end{array}$




\begin{tabular}{|c|c|c|c|}
\hline & 400 & & \\
\hline & 483049650 & 096664625 & 201 \\
\hline & 0 & 22 & 600 \\
\hline & 638808 & .002663 & 0.434 \\
\hline & 40 & 3.268 & -0.41 \\
\hline & 0. & 0 & 3300 \\
\hline & 1.8 & 0 & -0 \\
\hline & 1.1 & 0 & 500 \\
\hline & 1.849 & 0 & 2.3 \\
\hline & 0.4 & & \\
\hline & -0.5 & & \\
\hline & -1.8 & & \\
\hline & 0 & & \\
\hline & & & \\
\hline & & & \\
\hline & & & \\
\hline & & & \\
\hline & & & \\
\hline & & & \\
\hline & -2 & 0 & \\
\hline & -3.1 & 0 & -0 \\
\hline & -4.4 & 00 & \\
\hline & -1.3 & & \\
\hline & -0.3 & -0.1 & \\
\hline & -2.52 & -0.2 & -3 \\
\hline & -5.4 & -1.9 & \\
\hline & -2.8 & -1.3 & -3 \\
\hline & -3.3 & & \\
\hline & -4.4 & -0 & \\
\hline & -5.6 & & \\
\hline & -1 . & & \\
\hline & 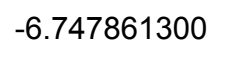 & & \\
\hline & $-c$ & & \\
\hline & & & \\
\hline & 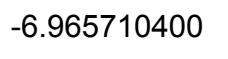 & 0.425 & \\
\hline & -5.799960000 & & \\
\hline & ת & & \\
\hline & & & \\
\hline & & & \\
\hline & -3.432787300 & & \\
\hline & & -1.505874900 & -3.795512300 \\
\hline & & & \\
\hline
\end{tabular}

\begin{tabular}{|c|c|c|c|}
\hline & & & \\
\hline & 812253200 & 2.489788800 & 10 \\
\hline & 0 & -3 & 50 \\
\hline & 980 & -2.1 & 0.63 \\
\hline & 0 & -4.0 & 50 \\
\hline & 78 & -3.4 & -2 \\
\hline & -2.70 & -4.8 & -12 \\
\hline & 0 & -2.5 & 00 \\
\hline & -4.51786 & -3.34 & 0.9 \\
\hline & -3.0 & & \\
\hline & 0.5 & -1 & \\
\hline & 1.0 & $-1 . \varepsilon$ & \\
\hline & -0.6 & -2 & \\
\hline & -0. & & \\
\hline & 1.2 & & \\
\hline & -1. & & \\
\hline & & & \\
\hline & & & \\
\hline & & & \\
\hline & & & \\
\hline & -4.969983 & 0 & \\
\hline & -8 & & \\
\hline & -2.6 & & \\
\hline & -4.0 & & \\
\hline & -1.4 & & \\
\hline & -2.5 & & \\
\hline & -2.43 & 0 & \\
\hline & -4.6 & & \\
\hline & -4.7 & & \\
\hline & -4.0 & & \\
\hline & -1 & & \\
\hline & & & \\
\hline & -0 & & \\
\hline & -3 & & -2 \\
\hline & -4 & & -3.7 \\
\hline & -5.67 & & -3 \\
\hline & -1.436 & & -2.76 \\
\hline & 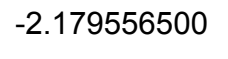 & & \\
\hline & & & \\
\hline & & & \\
\hline & & & -1.663347600 \\
\hline & & & \\
\hline
\end{tabular}


$\begin{array}{llll}H & -0.508086000 & 3.257316800 & -2.542866500\end{array}$

\section{C4-TS}

\begin{tabular}{|c|c|c|c|}
\hline 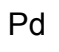 & 2.384154156 & 1.451865107 & 1.66 \\
\hline & 2.258213390 & 014923184 & .826381 \\
\hline & -1.275331024 & 372374379 & 192019 \\
\hline & 0 & 69 & \\
\hline & -0.9 & 38 & \\
\hline & -14 & 79 & 4.1 \\
\hline & -3.48 & 94 & .87 \\
\hline & -2.8 & & \\
\hline & & 2 & \\
\hline & 1.0 & 5 & \\
\hline & 1. & & \\
\hline & & & \\
\hline & & & \\
\hline & & & \\
\hline & & & \\
\hline & & & \\
\hline & & & \\
\hline & 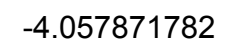 & & \\
\hline & -3.2233432 & & \\
\hline & -4.1 & & \\
\hline & -2.0 & 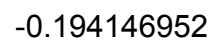 & \\
\hline & -3.5 & & \\
\hline & -3.38 & & \\
\hline & -0.00 & & \\
\hline & 1.2 & -0.4 & 1.74 \\
\hline & -0.41 & -0.4 & 1.2 \\
\hline & 1. & & \\
\hline & 0. & & \\
\hline & 2.00 & & \\
\hline 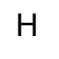 & 1. & & \\
\hline & 2. & 4 & \\
\hline & 1.12 & 2 & $3.2764 C$ \\
\hline & $0<4$ & 9 &. \\
\hline & $-0.376 \varepsilon$ & 2 & 2.12237 \\
\hline & 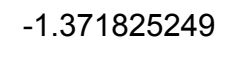 & 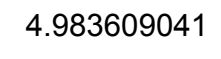 & 3.098551 \\
\hline & -0.1 & 6 & 0.0 \\
\hline & & & \\
\hline
\end{tabular}

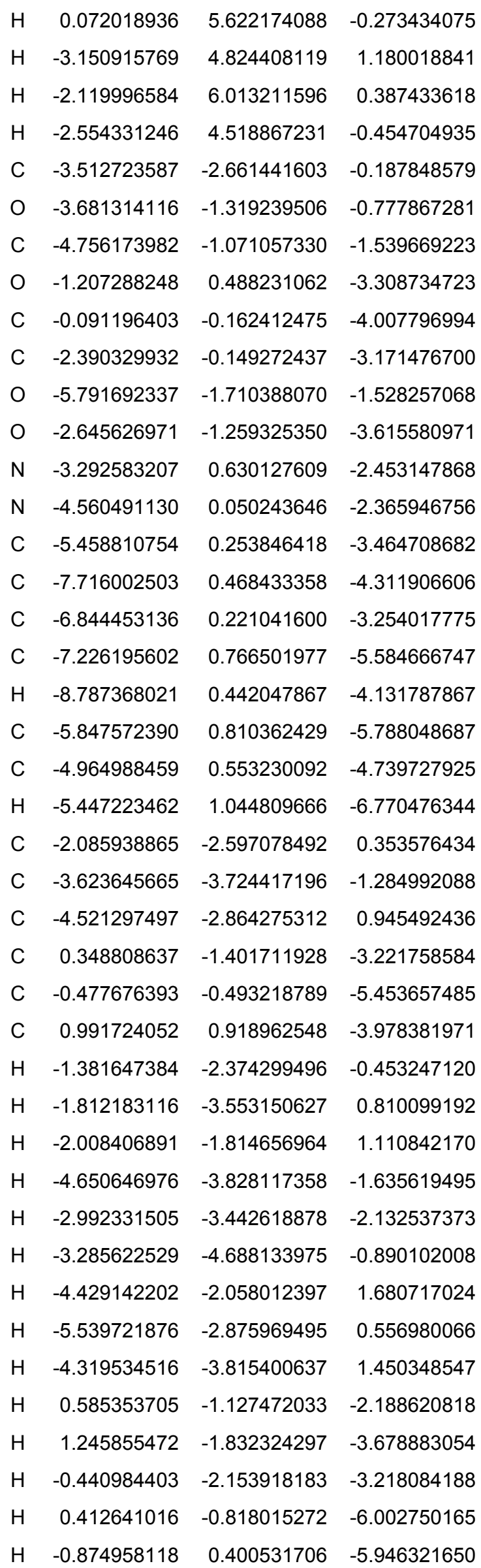




$\begin{array}{llll}\text { H } & -1.229600760 & -1.280740993 & -5.491579066 \\ \text { H } & 1.908676076 & 0.545347462 & -4.444503134 \\ \text { H } & 0.661131195 & 1.807683901 & -4.525138131 \\ \text { H } & 1.216760412 & 1.210248587 & -2.949341271 \\ \text { H } & -7.230205502 & 0.006812449 & -2.266993015 \\ \text { H } & -3.900492262 & 0.599049448 & -4.919676668 \\ \text { H } & -7.910259672 & 0.967207292 & -6.404250399 \\ \text { C } & -5.441392764 & 4.708039208 & -3.775302720 \\ \text { C } & -4.420964972 & 4.422161601 & -4.905456845 \\ \text { C } & -3.031578183 & 5.037358036 & -4.686925300 \\ \text { H } & -4.810982216 & 4.798944816 & -5.859158162 \\ \text { H } & -4.323495797 & 3.337860021 & -5.026287135 \\ \text { C } & -5.707839053 & 3.521223671 & -2.831346222 \\ \text { H } & -6.407820751 & 4.958517490 & -4.229797233 \\ \text { H } & -5.147057016 & 5.600293302 & -3.204777960 \\ \text { C } & -2.434454070 & 4.736405496 & -3.298826481 \\ \text { H } & -3.090031447 & 6.129409279 & -4.800103381 \\ \text { H } & -2.349470227 & 4.677792268 & -5.469005901 \\ \text { C } & -4.631951232 & 3.100596496 & -1.796726184 \\ \text { H } & -5.976622395 & 2.662417468 & -3.443267313 \\ \text { H } & -6.601311128 & 3.754971831 & -2.235822598 \\ \text { C } & -2.335675137 & 3.268618087 & -2.946091528 \\ \text { H } & -3.030212715 & 5.264315313 & -2.544800521 \\ \text { H } & -1.428498833 & 5.172100896 & -3.240575121 \\ \text { C } & -3.276440702 & 2.610220286 & -2.249508684 \\ \text { H } & -5.083829028 & 2.327464964 & -1.166717462 \\ \text { H } & -4.435812548 & 3.945534526 & -1.121879274 \\ \text { H } & -1.429132408 & 2.746497089 & -3.226500356\end{array}$

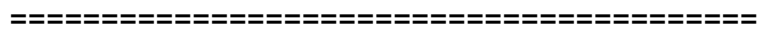

C5

\begin{tabular}{|c|c|c|c|}
\hline $\mathrm{Pd}$ & -2.692189089 & 1.806682407 & -0.944091602 \\
\hline $\mathrm{C}$ & -3.390264740 & 2.333702283 & 2.360975349 \\
\hline $\mathrm{P}$ & -1.941525655 & 2.700716554 & 1.125541318 \\
\hline $\mathrm{C}$ & -0.441062167 & 1.549908766 & 1.564760298 \\
\hline C & -1.381531487 & 4.538593408 & 1.329935821 \\
\hline $\mathrm{C}$ & -2.976506654 & 2.179047816 & 3.837499276 \\
\hline C & -4.441102801 & 3.460366747 & 2.249709922 \\
\hline $\mathrm{C}$ & -4.115572225 & 1.043929507 & 1.896250119 \\
\hline $\mathrm{C}$ & -0.954505991 & 0.110087404 & 1.789366474 \\
\hline $\mathrm{C}$ & 0.494903083 & 1.477173548 & 0.330035862 \\
\hline
\end{tabular}

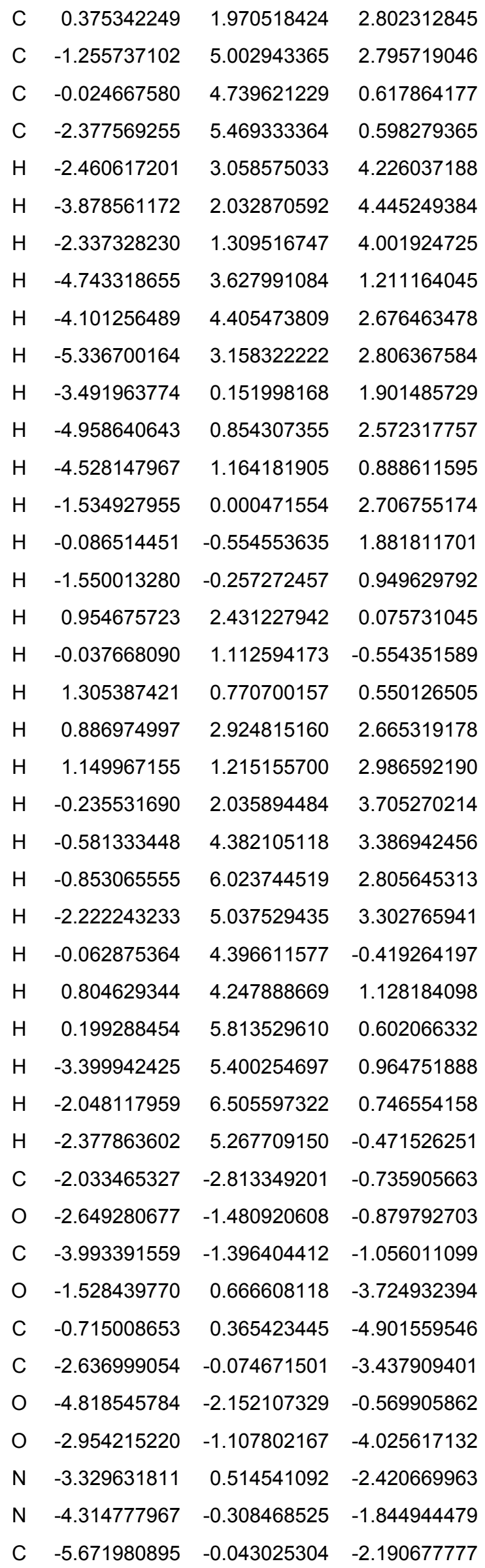




\begin{tabular}{|c|c|c|c|}
\hline & 1 & & \\
\hline & 638509083 & 160458100 & 2035045 \\
\hline & 8 & 5 & -2.92 \\
\hline & 50257 & 646582934 & 80 \\
\hline & 37 & 398 & -3.90 \\
\hline & 6.0 & 20 & 09 \\
\hline & 7.56 & 26 & 37 \\
\hline & -0.55 & 42 & 13 \\
\hline & -2.5774 & -3.750 & 1.8 \\
\hline & -2.2 & 6 & \\
\hline & -0.1 & 00 & \\
\hline & -1.5 & & \\
\hline & & & \\
\hline & 0. & & \\
\hline & & & \\
\hline & & & \\
\hline & & & \\
\hline & & & \\
\hline & & & \\
\hline & 3 & -2 & \\
\hline & -33 & -3 & \\
\hline & 1 & -4 & \\
\hline & 0.4 & -1.1 & \\
\hline & 0.58 & -1.1 & \\
\hline & -0.8 & -1.8 & \\
\hline & 1 & & \\
\hline & -1.9 & 55 & \\
\hline & -2.3 & -0.2 & \\
\hline & 1.0 & & \\
\hline & -0.0 & 3 & \\
\hline & & & \\
\hline & -0 & & \\
\hline & -5. & -0 & \\
\hline & -9.2 & & \\
\hline & 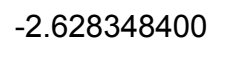 & & \\
\hline & -1.48 & & \\
\hline & & & \\
\hline & & & \\
\hline & & & \\
\hline & -3.754281078 & & \\
\hline & -4.464257270 & & - \\
\hline & 30405 & +5043 & \\
\hline
\end{tabular}

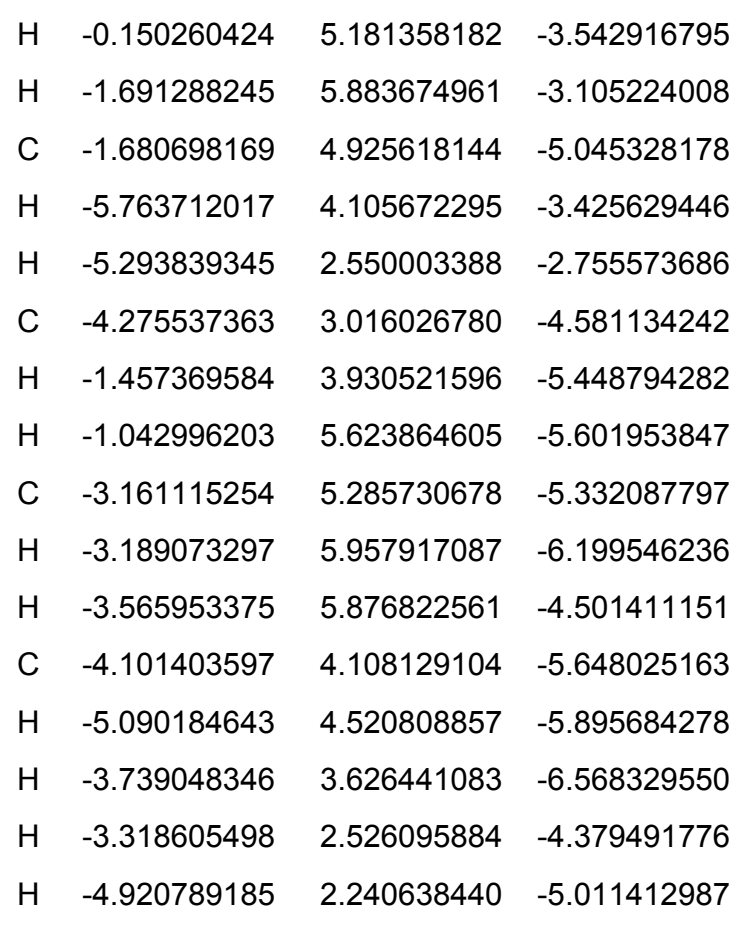

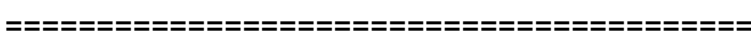

C5-TS

\begin{tabular}{cccc}
\multicolumn{5}{c}{$==================================$} \\
$\mathrm{Pd}$ & -2.415831319 & 1.453369083 & -0.632136161 \\
$\mathrm{C}$ & -2.141092120 & 2.063157898 & 2.853605923 \\
$\mathrm{P}$ & -1.210307615 & 2.352363853 & 1.175050852 \\
C & 0.508271473 & 1.443310250 & 1.262556636 \\
C & -0.858155678 & 4.249866054 & 0.993255474 \\
C & -1.289276329 & 2.186646976 & 4.131436603 \\
C & -3.319259882 & 3.057725437 & 2.938816385 \\
C & -2.786122108 & 0.655964316 & 2.814042771 \\
C & 0.282935251 & 0.003700113 & 1.770568012 \\
C & 1.045765234 & 1.289554928 & -0.181357481 \\
C & 1.587714273 & 2.110944295 & 2.135495201 \\
C & -0.346280886 & 4.963176403 & 2.259204842 \\
C & 0.158520250 & 4.448587428 & -0.153026949 \\
C & -2.152163444 & 4.949334242 & 0.518269740 \\
H & -0.807725925 & 3.161629670 & 4.225149506 \\
H & -1.936090411 & 2.054556367 & 5.008674970 \\
H & -0.514968032 & 1.418520081 & 4.188712486 \\
H & -3.928171644 & 3.034060311 & 2.029214535 \\
H & -2.995936890 & 4.084107803 & 3.120977104 \\
H & -3.965037932 & 2.770151807 & 3.777858028 \\
H & -2.056305122 & -0.151419496 & 2.853171635 \\
H & -3.438898277 & 0.542382293 & 3.689220141
\end{tabular}




\begin{tabular}{|c|c|c|c|}
\hline & 9 & & \\
\hline & .006077006 & .041019437 & 825 \\
\hline & 79 & 96 & 9101 \\
\hline & 480822086 & 28042 & 161 \\
\hline & 19 & 2.23 & -0.63 \\
\hline & 0.2 & 91 & -0.8 \\
\hline & 1.93 & 4 & -0 \\
\hline & 1.8 & 4 & 73 \\
\hline & 2.48 & 6 & 2.1 \\
\hline & 1.2 & & \\
\hline & 0.5 & & \\
\hline & -0.1 & & \\
\hline & -1. & & \\
\hline & & & \\
\hline & & & \\
\hline & & & \\
\hline & & & \\
\hline & & & \\
\hline & & & \\
\hline & 4 & 7 & \\
\hline & -3.8 & 1 & -0 \\
\hline & -4.8 & -0.9 & -1 \\
\hline & -1.18 & & \\
\hline & -0.08 & -0.2 & \\
\hline & -2.36 & -0.2 & -3 \\
\hline & -5.97 & -1.5 & -1. \\
\hline & $-2.5 \mathrm{~s}$ & -1.3 & \\
\hline & -3.2 & & \\
\hline & -4.5 & & \\
\hline & -5.2 & & \\
\hline & -1 & & \\
\hline & -6.6 & -0 & -3.8 \\
\hline & -0 & & \\
\hline & 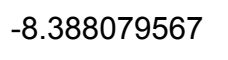 & -5 & \\
\hline & - & & \\
\hline & & & \\
\hline & & & \\
\hline & & & \\
\hline & & & \\
\hline & 8 & & \\
\hline & & & 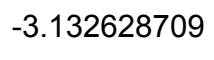 \\
\hline & & 4924 & \\
\hline
\end{tabular}

\begin{tabular}{lrrr}
$\mathrm{C}$ & 0.997381077 & 0.843395737 & -3.974863807 \\
$\mathrm{H}$ & -1.645093321 & -2.357536845 & -0.311926883 \\
$\mathrm{H}$ & -2.155508198 & -3.439651341 & 1.007463570 \\
$\mathrm{H}$ & -2.263050252 & -1.678558220 & 1.207535323 \\
$\mathrm{H}$ & -4.951363935 & -3.698143394 & -1.490475291 \\
$\mathrm{H}$ & -3.265503207 & -3.410729505 & -1.964800222 \\
$\mathrm{H}$ & -3.643815379 & -4.581050597 & -0.673473794 \\
$\mathrm{H}$ & -4.717863224 & -1.784058333 & 1.744360961 \\
$\mathrm{H}$ & -5.840689420 & -2.591759786 & 0.625526771 \\
$\mathrm{H}$ & -4.696325682 & -3.553713909 & 1.592762484 \\
$\mathrm{H}$ & 0.653743184 & -1.159822046 & -2.111817726 \\
$\mathrm{H}$ & 1.288874337 & -1.881290523 & -3.603754580 \\
$\mathrm{H}$ & -0.380893671 & -2.222164732 & -3.093971454 \\
$\mathrm{H}$ & 0.347047649 & -0.983168192 & -5.918653076 \\
$\mathrm{H}$ & -0.908017928 & 0.269425697 & -5.870360760 \\
$\mathrm{H}$ & -1.288430104 & -1.384657464 & -5.342142809 \\
$\mathrm{H}$ & 1.890592675 & 0.466128840 & -4.482178473 \\
$\mathrm{H}$ & 0.635505405 & 1.721632402 & -4.518682945 \\
$\mathrm{H}$ & 1.274025073 & 1.152956950 & -2.964541791 \\
$\mathrm{H}$ & -4.783701293 & 6.822022099 & -4.312667904 \\
$\mathrm{H}$ & -7.228374446 & -0.301471334 & -2.910889526 \\
$\mathrm{H}$ & -3.527987023 & 0.699019928 & -4.861299761 \\
$\mathrm{H}$ & -7.123738238 & 0.337588521 & -7.161702306 \\
$\mathrm{C}$ & -2.382675306 & 3.224640314 & -3.040236020 \\
$\mathrm{C}$ & -3.264412893 & 2.635738231 & -2.213909846 \\
$\mathrm{C}$ & -4.556230639 & 3.203060441 & -1.678921195 \\
$\mathrm{H}$ & -1.561747066 & 2.613981025 & -3.396812041 \\
$\mathrm{C}$ & -2.294111877 & 4.710718583 & -3.358432652 \\
$\mathrm{H}$ & -4.313892009 & 4.255941881 & -1.479451991 \\
$\mathrm{H}$ & -4.780890135 & 2.762135437 & -0.701904001 \\
$\mathrm{C}$ & -5.842349582 & 3.131865824 & -2.540976145 \\
$\mathrm{H}$ & -1.254441632 & 4.884480056 & -3.661000616 \\
$\mathrm{H}$ & -2.428940363 & 5.297925627 & -2.439608512 \\
-3.211843881 & 5.330922078 & -4.445915871 \\
\hline & -6.570996065 & 3.811806823 & -2.076843010 \\
\hline & -5.259084114 & 2.130293837 & -2.432694250 \\
\hline
\end{tabular}




\section{$\begin{array}{llll}\text { H } & -6.722071727 & 5.367022085 & -4.052701513\end{array}$ \\ $\begin{array}{llll}\text { H } & -5.841080426 & 4.994979034 & -5.525680847\end{array}$ \\ $\begin{array}{llll}H & -4.796352345 & 3.010796687 & -4.425085505\end{array}$ \\ H $\quad-6.527468636 \quad 2.920148470 \quad-4.559689255$}

\section{D}

\begin{tabular}{cccc} 
====================================== \\
Pd & -2.322445587 & 1.996732074 & -1.444516464 \\
C & -2.633674340 & -0.611900359 & -3.705683016 \\
P & -2.801888527 & 1.320773499 & -3.541782146 \\
C & -4.621499943 & 1.805484366 & -4.036253902 \\
$\mathrm{C}$ & -1.593364799 & 2.141089989 & -4.829575386 \\
$\mathrm{C}$ & -3.278839149 & -1.239443647 & -4.956979148 \\
$\mathrm{C}$ & -1.135143797 & -0.980802886 & -3.681812189 \\
$\mathrm{C}$ & -3.241662264 & -1.270155807 & -2.443789280 \\
$\mathrm{C}$ & -5.601144338 & 0.886443381 & -3.276057747 \\
$\mathrm{C}$ & -4.909728758 & 3.236331133 & -3.521437194 \\
$\mathrm{C}$ & -4.948919374 & 1.740701946 & -5.540982590 \\
$\mathrm{C}$ & -1.550748664 & 1.489455118 & -6.225458042 \\
$\mathrm{C}$ & -1.976660558 & 3.628052993 & -4.984492119 \\
$\mathrm{C}$ & -0.164576845 & 2.143832734 & -4.234222792 \\
$\mathrm{H}$ & -2.894635777 & -0.819331160 & -5.887804450 \\
$\mathrm{H}$ & -3.061208256 & -2.315436913 & -4.969601313 \\
$\mathrm{H}$ & -4.365817215 & -1.135349785 & -4.959440544 \\
$\mathrm{H}$ & -0.625977410 & -0.513401894 & -2.833369764 \\
$\mathrm{H}$ & -0.614716118 & -0.705470119 & -4.600745687 \\
$\mathrm{H}$ & -1.040649834 & -2.068275287 & -3.572503891 \\
$\mathrm{H}$ & -4.317894993 & -1.132412169 & -2.356549665 \\
$\mathrm{H}$ & -3.051951832 & -2.350547633 & -2.486860826 \\
$\mathrm{H}$ & -2.776341133 & -0.872973652 & -1.536696663 \\
$\mathrm{H}$ & -5.607042096 & -0.136632897 & -3.655652854 \\
$\mathrm{H}$ & -6.618498308 & 1.279053895 & -3.396498011 \\
$\mathrm{H}$ & -5.375845585 & 0.866847534 & -2.205428044 \\
$\mathrm{H}$ & -4.320261844 & 4.004299661 & -4.019552903 \\
$\mathrm{H}$ & -4.719057580 & 3.308247355 & -2.446501034 \\
$\mathrm{H}$ & -5.967640585 & 3.466938276 & -3.702593207 \\
$\mathrm{H}$ & -4.391009207 & 2.480430104 & -6.118794457 \\
$\mathrm{H}$ & -6.014521352 & 1.962967502 & -5.683986024 \\
$\mathrm{H}$ & -4.761528413 & 0.756684508 & -5.973778315 \\
\hline & -2.529351283 & 1.454665448 & -6.706951301 \\
\hline & -0.888244390 & 2.077656638 & -6.873866505
\end{tabular}

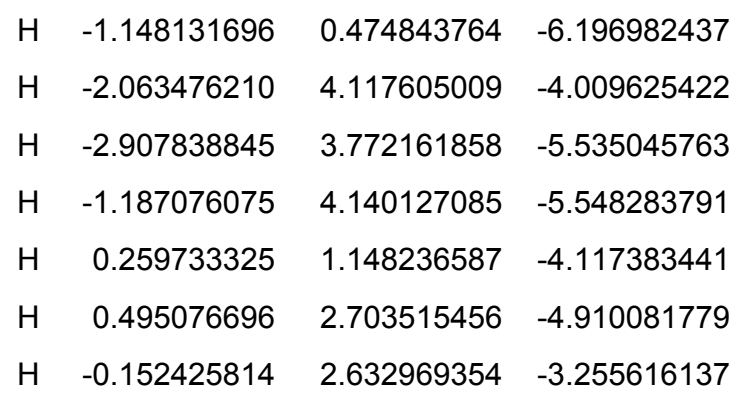

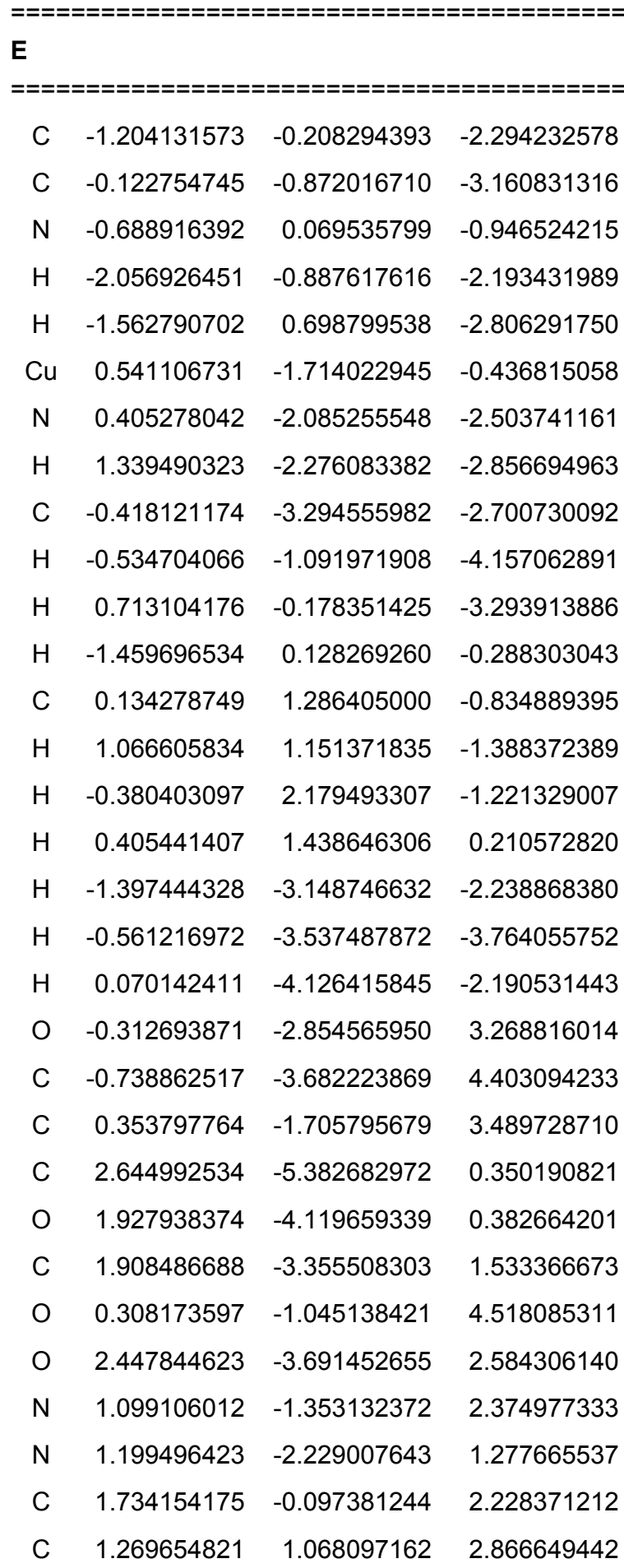




$\begin{array}{lrrr}\text { C } & 1.883345202 & 2.293718394 & 2.610735109 \\ \text { C } & 2.958544431 & 2.394831254 & 1.724622514 \\ \text { H } & 1.508527828 & 3.182421109 & 3.112333605 \\ \text { C } & 2.821974714 & 0.005191125 & 1.342608603 \\ \text { C } & 3.423976760 & 1.237511954 & 1.098603840 \\ \text { H } & 4.267953066 & 1.287637498 & 0.415244203 \\ \text { C } & -1.158736875 & -4.982127796 & 3.713260212 \\ \text { C } & -1.924811934 & -3.024796033 & 5.115190466 \\ \text { C } & 0.448039043 & -3.930758209 & 5.339879709 \\ \text { C } & 4.143299184 & -5.156955238 & 0.588185060 \\ \text { C } & 2.049018755 & -6.370858941 & 1.361401506 \\ \text { C } & 2.399355710 & -5.874979715 & -1.080619197 \\ \text { H } & -1.951284527 & -4.788691206 & 2.983232580 \\ \text { H } & -1.530993231 & -5.699251041 & 4.451723620 \\ \text { H } & -0.304889988 & -5.421389682 & 3.190555726 \\ \text { H } & -1.620959861 & -2.078013325 & 5.562245250 \\ \text { H } & -2.736458478 & -2.837711165 & 4.404017845 \\ \text { H } & -2.301655228 & -3.689353256 & 5.900501836 \\ \text { H } & 1.311247424 & -4.252711955 & 4.751129722 \\ \text { H } & 0.716086917 & -3.023543929 & 5.883097694 \\ \text { H } & 0.185979163 & -4.711661822 & 6.061556185 \\ \text { H } & 4.530829762 & -4.426474244 & -0.130258460 \\ \text { H } & 4.690878708 & -6.096281937 & 0.453837160 \\ \text { H } & 4.313112940 & -4.783258321 & 1.597848821 \\ \text { H } & 2.502715186 & -7.359603966 & 1.232106257 \\ \text { H } & 0.969708945 & -6.462434120 & 1.199969198 \\ \text { H } & 2.224580053 & -6.025266613 & 2.379848507 \\ \text { H } & 2.920379041 & -6.821214089 & -1.257433771 \\ \text { H } & 1.329261419 & -6.033919282 & -1.249706965 \\ \text { H } & 2.762356850 & -5.137992861 & -1.805031496 \\ \text { H } & 0.448933961 & 1.003190620 & 3.567105586 \\ \text { H } & 3.179824297 & -0.890030984 & 0.850460828 \\ \text { H } & 3.428264062 & 3.355336056 & 1.532976771\end{array}$

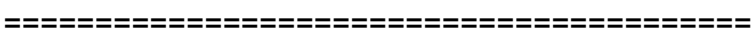

F1

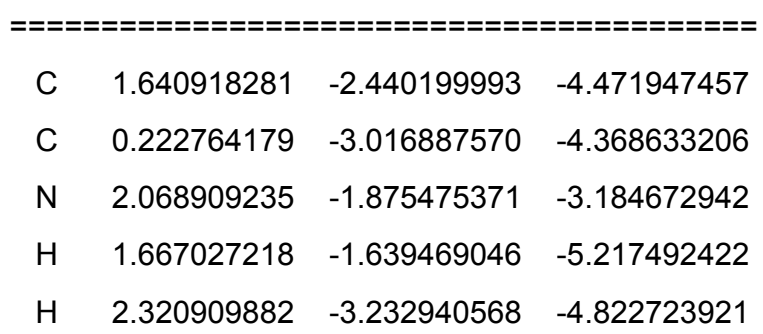

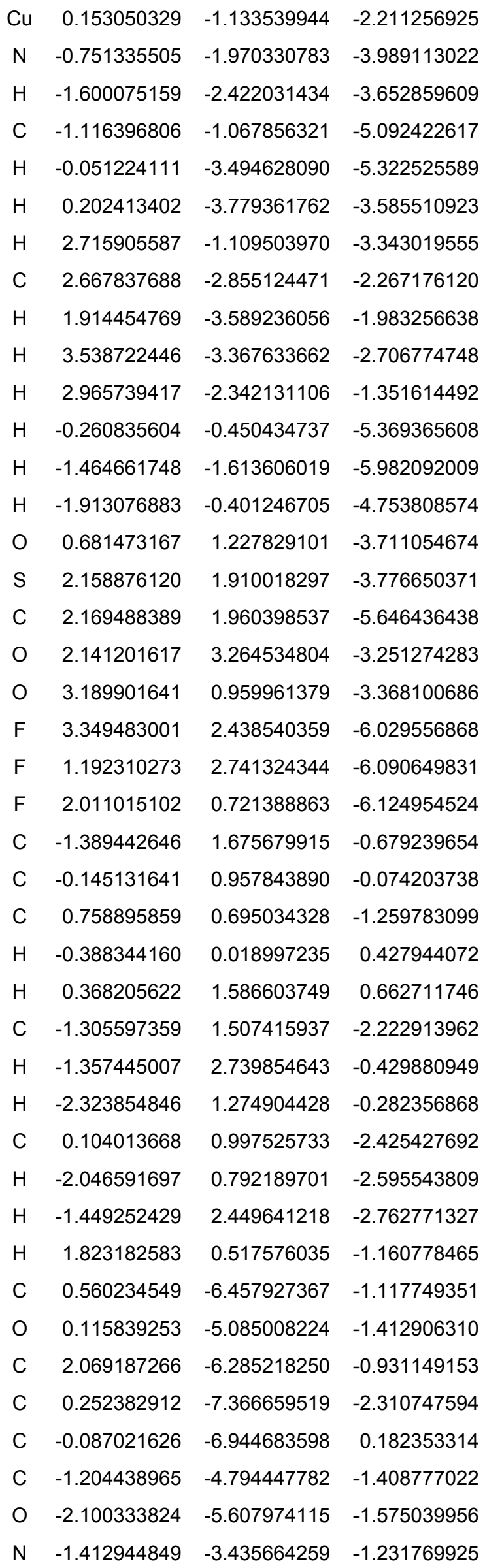




\begin{tabular}{lrrr} 
O & 1.208196566 & -1.928566977 & 0.536313272 \\
C & 1.997281765 & -1.969660334 & 1.757353613 \\
C & 0.167065119 & -2.815273618 & 0.335651675 \\
C & 1.118499069 & -1.673518484 & 2.978833611 \\
C & 2.715729318 & -3.319112452 & 1.885972089 \\
C & 3.011074495 & -0.841168295 & 1.541505284 \\
O & -0.168836985 & -3.667323385 & 1.156209673 \\
N & -0.353350229 & -2.571071289 & -0.890214413 \\
C & -2.669435241 & -2.823188700 & -1.458612124 \\
C & -3.598697809 & -3.329314727 & -2.388307486 \\
C & -2.945727102 & -1.603102351 & -0.820479566 \\
C & -4.764143192 & -2.615764368 & -2.665933325 \\
C & -5.034082881 & -1.399757834 & -2.035318322 \\
H & -5.468252426 & -3.021538977 & -3.387694346 \\
H & -3.416132824 & -4.283099749 & -2.864548109 \\
C & -4.118162542 & -0.906493799 & -1.104750809 \\
H & -5.944651388 & -0.851705751 & -2.258645264 \\
H & -4.318100354 & 0.029398181 & -0.589149258 \\
H & -2.228196208 & -1.216657661 & -0.109124128 \\
H & 2.532815037 & -5.899632484 & -1.844323929 \\
H & 2.529704488 & -7.248665762 & -0.691593817 \\
H & 2.269864612 & -5.585555744 & -0.115405389 \\
H & -0.824474159 & -7.448453644 & -2.459378884 \\
H & 0.708232139 & -6.963185383 & -3.221708497 \\
H & 0.667815674 & -8.364598867 & -2.133328168 \\
H & 0.028093066 & -6.177199074 & 0.952224232 \\
H & -1.150577361 & -7.142399174 & 0.043608932 \\
H & 0.404948630 & -7.866933606 & 0.508805853 \\
H & 0.596095298 & -0.720562631 & 2.841776861 \\
H & 1.740502989 & -1.597038405 & 3.877302726 \\
H & 0.380839341 & -2.463753542 & 3.117379003 \\
H & 3.399711418 & -3.300192813 & 2.741353192 \\
H & 3.301889093 & -3.520180813 & 0.982843166 \\
H & 1.991895857 & -4.122428234 & 2.023950744 \\
H & 3.682853100 & -0.760194729 & 2.401567037 \\
H & 3.614741966 & -1.030356103 & 0.647859044 \\
\hline & 2.496260587 & 0.116197964 & 1.412987396
\end{tabular}

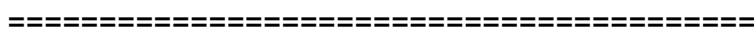

F1-TS

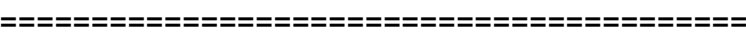

$\begin{array}{llll}\text { C } & 1.833438191 & -2.698954320 & -4.709837212\end{array}$

\begin{tabular}{|c|c|c|c|}
\hline & 247 & & \\
\hline & 1 & 2 & -345502746 \\
\hline & 4 & 36 & -5522 \\
\hline & 0 & -3 & \\
\hline & 6532 & & -2 \\
\hline & .54 & -2.1 & $-2-2>>$ \\
\hline & 313 & -2.4 & -4 \\
\hline & 2590 & -1.2 & -5.5 \\
\hline & 72 & -3.7 & -5.5 \\
\hline & 0.30 & -3.9 & \\
\hline & $2.8 \varepsilon$ & & \\
\hline & 2.80 & & \\
\hline & 2.0 & & \\
\hline & 3.682079507 & & \\
\hline & & & \\
\hline & & & \\
\hline & 078 & & \\
\hline & 5 & & \\
\hline & & & \\
\hline & & & \\
\hline & & & \\
\hline & & & \\
\hline & 8 & & \\
\hline & 3.2 & & \\
\hline & 1.1 & & \\
\hline & 2.50 & & \\
\hline & 0.51 & -6.3 & \\
\hline & 0.17 & -4 . & \\
\hline & 4 & & \\
\hline & 3 & & \\
\hline & & & \\
\hline & -1 & & \\
\hline & -2.06 & & \\
\hline & -1.2788 & 0.2 & -1.0 \\
\hline & 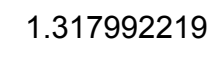 & & \\
\hline & 2.032803575 & -1.74 & \\
\hline & 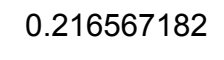 & & \\
\hline & & & \\
\hline & & & \\
\hline & & & \\
\hline & 02748379 & 1. & 0.94463005 \\
\hline & & & \\
\hline
\end{tabular}




\begin{tabular}{lrrr}
$\mathrm{C}$ & -2.582989215 & -2.664267652 & -1.549766173 \\
$\mathrm{C}$ & -3.499284258 & -2.985598920 & -2.560960801 \\
$\mathrm{C}$ & -2.933946165 & -1.731845429 & -0.568244645 \\
$\mathrm{C}$ & -4.744455217 & -2.359288643 & -2.597188782 \\
$\mathrm{C}$ & -5.090480967 & -1.414682129 & -1.629032763 \\
$\mathrm{H}$ & -5.446670718 & -2.613783181 & -3.386368069 \\
$\mathrm{H}$ & -3.245698528 & -3.749938407 & -3.287343580 \\
$\mathrm{C}$ & -4.182979868 & -1.114682082 & -0.610704199 \\
$\mathrm{H}$ & -6.060539066 & -0.927178545 & -1.660392791 \\
$\mathrm{H}$ & -4.451646780 & -0.398865935 & 0.161685996 \\
$\mathrm{H}$ & -2.230229929 & -1.517896002 & 0.227152666 \\
$\mathrm{H}$ & 2.540715344 & -5.969106097 & -1.796178161 \\
$\mathrm{H}$ & 2.406434067 & -7.214224409 & -0.538859748 \\
$\mathrm{H}$ & 2.242810506 & -5.496469951 & -0.109414492 \\
$\mathrm{H}$ & -0.889764307 & -7.359102284 & -2.406768005 \\
$\mathrm{H}$ & 0.694990937 & -7.040078899 & -3.149262022 \\
$\mathrm{H}$ & 0.528893324 & -8.330190615 & -1.941859395 \\
$\mathrm{H}$ & -0.082190376 & -5.824609766 & 0.904420910 \\
$\mathrm{H}$ & -1.281166928 & -6.803365847 & 0.039451274 \\
$\mathrm{H}$ & 0.206470873 & -7.567228255 & 0.642583561 \\
$\mathrm{H}$ & 0.708227100 & -0.271245557 & 2.403831417 \\
$\mathrm{H}$ & 1.704375198 & -1.139721862 & 3.588509086 \\
$\mathrm{H}$ & -2.300658509 & 0.449598289 & -2.222267330 \\
$\mathrm{H}$ & 0.305469550 & -1.947964099 & 2.836053387 \\
$\mathrm{H}$ & 3.207043278 & -3.136895228 & 2.711070943 \\
$\mathrm{H}$ & 3.200556699 & -3.483479542 & 0.970668326 \\
$\mathrm{H}$ & 1.767686112 & -3.861692401 & 1.955589443 \\
$\mathrm{H}$ & -0.839659602 & -0.736656086 & 2.177244597 \\
$\mathrm{H}$ & 3.780172624 & -1.069097251 & 0.433486351 \\
$\mathrm{H}$ & 2.808675771 & 0.244284117 & 1.122090772 \\
$\mathrm{C}$ & -1.832393187 & 1.692563283 & -0.443274305 \\
$\mathrm{C}$ & -0.425627025 & 1.930545244 & 0.184683237 \\
$\mathrm{H}$ & -1.583680054 & -2.209560138 & -1.905287386 \\
\hline & -2.343215742 & 0.8979827959 & 0.102677870 \\
$\mathrm{H}$ & -2.034246276 & -2.626894956 \\
\hline
\end{tabular}

\begin{tabular}{llll} 
========================================= \\
F2-TS & & & \\
======================================= \\
C & 1.870433725 & -2.705973902 & -4.686438659 \\
C & 0.422042103 & -3.208637110 & -4.633201310 \\
N & 2.250274895 & -2.046052059 & -3.424321659 \\
H & 1.979428296 & -1.981090915 & -5.496174508 \\
H & 2.534621158 & -3.553154418 & -4.915860018 \\
$\mathrm{Cu}$ & 0.424310061 & -1.119167816 & -2.581631998 \\
$\mathrm{~N}$ & -0.515231027 & -2.096151781 & -4.397979868 \\
$\mathrm{H}$ & -1.399059975 & -2.469625812 & -4.066277035 \\
$\mathrm{C}$ & -0.744965553 & -1.224394975 & -5.554310122 \\
$\mathrm{H}$ & 0.193488286 & -3.748597607 & -5.566935269 \\
$\mathrm{H}$ & 0.317596764 & -3.908688402 & -3.800720728 \\
$\mathrm{H}$ & 2.911808384 & -1.296042126 & -3.605016653 \\
$\mathrm{C}$ & 2.802651257 & -2.957492473 & -2.410467107 \\
$\mathrm{H}$ & 2.025704210 & -3.654430529 & -2.097988582 \\
$\mathrm{H}$ & 3.677104242 & -3.515735952 & -2.779537825 \\
$\mathrm{H}$ & 3.081297668 & -2.370461833 & -1.534754347 \\
$\mathrm{H}$ & 0.140093201 & -0.613346761 & -5.742781169 \\
$\mathrm{H}$ & -0.994945192 & -1.781928509 & -6.470704617 \\
$\mathrm{H}$ & -1.565353663 & -0.540577307 & -5.322171982 \\
$\mathrm{O}$ & 0.641417305 & 0.754532120 & -3.453211370 \\
$\mathrm{C}$ & 1.949209630 & 1.623273455 & -3.378629024 \\
$\mathrm{C}$ & 2.356022938 & 1.560331821 & -5.199585869 \\
$\mathrm{C}$ & 2.070047192 & -1.733399353 & 1.466342497 \\
$\mathrm{C}$ & 0.210636436 & -2.529604280 & 0.063520038 \\
$\mathrm{O}$ & 1.221676891 & -1.363054397 & 2.688959648 \\
$\mathrm{~F}$ & 3.034085026 & 0.908788291 & -2.696641230 \\
$\mathrm{~F}$ & 3.430679957 & 2.309818485 & -5.434548085 \\
$\mathrm{~F}$ & 1.330748435 & 2.001902512 & -5.927555465 \\
$\mathrm{C}$ & 2.625208916 & 0.289505905 & -5.554730333 \\
$\mathrm{O}$ & 0.449022191 & -6.355048982 & -1.144460242 \\
$\mathrm{C}$ & 0.118181735 & -4.985512746 & -1.577986807 \\
$\mathrm{C}$ & 1.962614674 & -6.283013974 & -0.934218350 \\
\hline & -0.2690748390 & -7.352233593 & -2.249125690 \\
\hline
\end{tabular}




\begin{tabular}{|c|c|c|c|}
\hline & 0 & 04836 & \\
\hline & 3.148977214 & 0.674185031 & 200073284 \\
\hline & 0.228377262 & -3.2 & 34 \\
\hline & -0.234966303 & -2.396335175 & 209258124 \\
\hline & -2.617116411 & -2.668291881 & -1.47771200 \\
\hline & -3.521716441 & -2.85 & 380 \\
\hline & -2.973674645 & -1.8 & 62 \\
\hline & -4.7604 & -2.2 & 5358 \\
\hline & -5.108407534 & -1.390226947 & -1.4 \\
\hline & -5.45517 & -2. & -3 \\
\hline & -3.2 & -3.5 & 934 \\
\hline & -4.2 & -1 & $-c$ \\
\hline & -6.0 & -0 & \\
\hline & -4 & & \\
\hline & -2.2 & & \\
\hline & & & \\
\hline & & & \\
\hline & & & \\
\hline & 4 & & \\
\hline & 0.5 & -7.0 & \\
\hline & 0.4 & -8.3 & -1. \\
\hline & -0.1 & -5.8 & 966 \\
\hline & -1.3 & -6.7 & \\
\hline & 0.14 & -7.5 & \\
\hline & 0.751 & -0.3 & 2.5 \\
\hline & 1.8595 & -1.2 & 82 \\
\hline & 0.44 & -2.1 & \\
\hline & 3.38 & -3.1 & \\
\hline & 3.2 & -3.3 & \\
\hline & 9 & -3.8 & \\
\hline & 3.8 & -0 & \\
\hline & 1 & -0 & \\
\hline & 2.701099405 & 1 & \\
\hline & -2 & 0 & -1 \\
\hline & -2.00 & - & \\
\hline & -0.48 & & \\
\hline & -2 & & \\
\hline & & & \\
\hline & & & \\
\hline & -2.008429648 & & -1.597560179 \\
\hline & & & \\
\hline & 28148658 & 1.09951421 & $.103 / 108$ \\
\hline
\end{tabular}

$\begin{array}{llll}\text { H } & -0.228825887 & 1.252623334 & 1.314608124 \\ \text { H } & -0.129374241 & 2.782440057 & 0.449488868 \\ \text { C } & -0.375166742 & 0.712645518 & -1.846228071 \\ \text { H } & -1.788837210 & 1.313489390 & -3.305227977 \\ \text { H } & -2.340591326 & 0.016593178 & -2.233383290 \\ \text { H } & 1.349835349 & 0.954999519 & -0.658075908\end{array}$

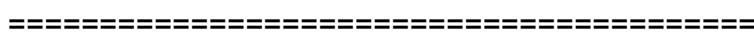

F3-TS

\begin{tabular}{cccc}
\multicolumn{1}{c}{$===================================$} \\
$\mathrm{C}$ & 2.277670751 & -2.530854260 & -4.432173775 \\
$\mathrm{C}$ & 0.973261416 & -3.337918827 & -4.473752205 \\
$\mathrm{~N}$ & 2.452539303 & -1.866457925 & -3.128918074 \\
$\mathrm{H}$ & 2.255072794 & -1.751416867 & -5.197848205 \\
$\mathrm{H}$ & 3.121729507 & -3.199705802 & -4.665400044 \\
$\mathrm{Cu}$ & 0.436881491 & -1.173678039 & -2.507516888 \\
$\mathrm{~N}$ & -0.196805948 & -2.463895813 & -4.278506294 \\
$\mathrm{H}$ & -0.958384625 & -3.012944338 & -3.894886473 \\
$\mathrm{C}$ & -0.659949062 & -1.769053028 & -5.483559484 \\
$\mathrm{H}$ & 0.918833384 & -3.889672814 & -5.426316112 \\
$\mathrm{H}$ & 0.970301586 & -4.069466022 & -3.661496002 \\
$\mathrm{H}$ & 3.041081608 & -1.044988035 & -3.244939907 \\
$\mathrm{C}$ & 2.986980454 & -2.741118578 & -2.074670488 \\
$\mathrm{H}$ & 2.241110952 & -3.494717377 & -1.819549074 \\
$\mathrm{H}$ & 3.926508683 & -3.234503706 & -2.369890070 \\
$\mathrm{H}$ & 3.150615671 & -2.145584059 & -1.175814731 \\
$\mathrm{H}$ & 0.077694523 & -1.029162515 & -5.794126176 \\
$\mathrm{H}$ & -0.849213464 & -2.453839962 & -6.325044302 \\
$\mathrm{H}$ & -1.583488947 & -1.234973369 & -5.246263087 \\
$\mathrm{O}$ & 0.471465026 & 0.618628933 & -3.602632405 \\
$\mathrm{~S}$ & 1.612943130 & 1.653434579 & -3.889599751 \\
$\mathrm{C}$ & 1.544405218 & 1.602333700 & -5.755282916 \\
$\mathrm{O}$ & 1.240941053 & 3.010989738 & -3.503708227 \\
$\mathrm{O}$ & 2.930831111 & 1.126795274 & -3.511961380 \\
$\mathrm{~F}$ & 2.377278569 & 2.521625588 & -6.239185883 \\
$\mathrm{~F}$ & 0.306348570 & 1.846616535 & -6.183592854 \\
$\mathrm{~F}$ & 1.928563212 & 0.392449154 & -6.198617332 \\
$\mathrm{C}$ & 0.608398696 & -6.289238297 & -1.044628704 \\
$\mathrm{O}$ & 0.243247197 & -4.921781838 & -1.458187531 \\
$\mathrm{C}$ & 2.093332819 & -6.151158541 & -0.704258266 \\
$\mathrm{C}$ & 0.399431527 & -7.265487200 & -2.205778986 \\
$\mathrm{C}$ & -0.194852118 & -6.669797072 & 0.203446218
\end{tabular} 


\begin{tabular}{llll}
$\mathrm{C}$ & -1.065894985 & -4.619448034 & -1.625821809 \\
$\mathrm{O}$ & -1.946948700 & -5.421758749 & -1.894605418 \\
$\mathrm{~N}$ & -1.291648415 & -3.263041946 & -1.497985122 \\
$\mathrm{O}$ & 1.137744801 & -1.674147479 & 0.468639463 \\
$\mathrm{C}$ & 1.815274612 & -1.735121228 & 1.760599079 \\
$\mathrm{C}$ & 0.082642214 & -2.516439298 & 0.201977521 \\
$\mathrm{C}$ & 0.862338263 & -1.330081473 & 2.891779207 \\
$\mathrm{C}$ & 2.407045166 & -3.132204339 & 1.987119034 \\
$\mathrm{C}$ & 2.939714341 & -0.705025161 & 1.611970897 \\
$\mathrm{O}$ & -0.415263328 & -3.275275916 & 1.031425588 \\
$\mathrm{~N}$ & -0.281030439 & -2.369635185 & -1.097568319 \\
$\mathrm{C}$ & -2.611135876 & -2.742667137 & -1.608929786 \\
$\mathrm{C}$ & -3.412785738 & -3.024445846 & -2.722021194 \\
$\mathrm{C}$ & -3.086713308 & -1.885928620 & -0.610710565 \\
$\mathrm{C}$ & -4.666579367 & -2.427861394 & -2.844752610 \\
$\mathrm{C}$ & -5.134634656 & -1.552403368 & -1.862032469 \\
$\mathrm{H}$ & -5.280160473 & -2.648701060 & -3.713872970 \\
$\mathrm{H}$ & -3.068130873 & -3.734040968 & -3.465864512 \\
$\mathrm{C}$ & -4.343284768 & -1.296310685 & -0.739223425 \\
$\mathrm{H}$ & -6.111161571 & -1.086853149 & -1.962850763 \\
$\mathrm{H}$ & -2.531344700 & 0.290017824 & 1.418455637 \\
$\mathrm{H}$ & -4.707758115 & -0.639876011 & 0.046806677 \\
$\mathrm{H}$ & -2.472356161 & -1.713982881 & 0.265345306 \\
$\mathrm{H}$ & 2.665629844 & -5.830638338 & -1.580368632 \\
$\mathrm{H}$ & 2.490056276 & -7.114116260 & -0.368035313 \\
$\mathrm{H}$ & 2.232963619 & -5.416576812 & 0.092811936 \\
$\mathrm{H}$ & -0.657107735 & -7.332658698 & -2.464127654 \\
$\mathrm{H}$ & 0.960801732 & -6.934043714 & -3.086341381 \\
$\mathrm{H}$ & 0.764607518 & -8.258573733 & -1.921841876 \\
$\mathrm{H}$ & -0.143543960 & -5.854438954 & 0.930564348 \\
$\mathrm{H}$ & -1.242252621 & -6.849014392 & -0.042685472 \\
$\mathrm{H}$ & 0.224837118 & -7.578897217 & 0.646217604 \\
$\mathrm{H}$ & 0.431001581 & -0.344400360 & 2.686284050 \\
$\mathrm{H}$ & 1.411121385 & -1.270340576 & 3.837888623 \\
\hline & 0.054901147 & -2.055178047 & 2.988362860 \\
$\mathrm{H}$ & 3.010363429 & -3.137294925 & 2.901057242 \\
$\mathrm{H}$ & 2.814383193 & -0.042171443 \\
\hline
\end{tabular}

$\begin{array}{lrrr}\text { C } & -0.320202009 & 3.158226029 & -0.013453990 \\ \text { H } & -2.347434361 & 3.589647008 & -0.617059256 \\ \text { H } & -2.228761966 & 2.865561774 & 0.977408901 \\ \text { C } & -1.547054324 & 1.084691556 & -1.971927972 \\ \text { H } & -3.271511672 & 1.362011158 & -0.729366162 \\ \text { H } & -1.915978761 & 0.645215510 & 0.106518322 \\ \text { C } & 0.602983461 & 1.964212718 & 0.297662969 \\ \text { H } & -0.153471027 & 3.945253516 & 0.731255701 \\ \text { H } & -0.008970762 & 3.573409084 & -0.978855238 \\ \text { C } & -0.113438373 & 0.764959053 & -1.777754651 \\ \text { H } & -1.640848989 & 1.917008153 & -2.679261672 \\ \text { H } & -2.062268121 & 0.218889246 & -2.399122456 \\ \text { C } & 0.848881534 & 1.100824616 & -0.931894743 \\ \text { H } & 0.210308232 & 1.359911816 & 1.124624177 \\ \text { H } & 1.581933738 & 2.332281479 & 0.622506833 \\ \text { H } & 1.869621194 & 0.796117936 & -1.136200246\end{array}$

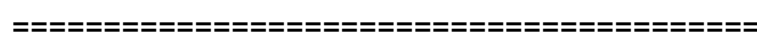

\section{F4-TS}

\begin{tabular}{|c|c|c|c|}
\hline $\mathrm{C}$ & 1.826459714 & -2.729259009 & -4.763091029 \\
\hline C & 0.379529619 & -3.232514847 & -4.692409659 \\
\hline $\mathrm{N}$ & 2.215117952 & -2.075214005 & -3.502289435 \\
\hline $\mathrm{H}$ & 1.927740682 & -2.002232684 & -5.573019258 \\
\hline $\mathrm{H}$ & 2.487847380 & -3.575897164 & -5.002439546 \\
\hline $\mathrm{Cu}$ & 0.372371869 & -1.152079776 & -2.650396668 \\
\hline $\mathrm{N}$ & -0.556698638 & -2.111322168 & -4.482442646 \\
\hline $\mathrm{H}$ & -1.453695922 & -2.480162880 & -4.178471610 \\
\hline C & -0.737917761 & -1.251125517 & -5.658707385 \\
\hline $\mathrm{H}$ & 0.145119501 & -3.792575427 & -5.612564050 \\
\hline $\mathrm{H}$ & 0.277641266 & -3.911759189 & -3.841852816 \\
\hline $\mathrm{H}$ & 2.873561507 & -1.323982108 & -3.683767519 \\
\hline C & 2.787790710 & -2.995594586 & -2.508026809 \\
\hline $\mathrm{H}$ & 2.018491084 & -3.698096697 & -2.188958200 \\
\hline $\mathrm{H}$ & 3.656640285 & -3.546495499 & -2.900966668 \\
\hline $\mathrm{H}$ & 3.079444276 & -2.416993265 & -1.632629520 \\
\hline $\mathrm{H}$ & 0.134720791 & -0.606082977 & -5.786045779 \\
\hline $\mathrm{H}$ & -0.899107577 & -1.822409726 & -6.585982459 \\
\hline $\mathrm{H}$ & -1.600758367 & -0.599482529 & -5.500551073 \\
\hline $\mathrm{O}$ & 0.635854038 & 0.767001525 & -3.415176263 \\
\hline$S$ & 1.950083732 & 1.589238346 & -3.212831829 \\
\hline C & 2.499175464 & 1.559474988 & -4.997610873 \\
\hline
\end{tabular}




\begin{tabular}{lrrr}
$\mathrm{O}$ & 1.682890234 & 2.985941219 & -2.883514087 \\
$\mathrm{O}$ & 2.953426353 & 0.836672988 & -2.454208432 \\
$\mathrm{~F}$ & 3.626226679 & 2.255322438 & -5.128165094 \\
$\mathrm{~F}$ & 1.555597398 & 2.077131341 & -5.784437267 \\
$\mathrm{~F}$ & 2.728135286 & 0.287039671 & -5.380800315 \\
$\mathrm{C}$ & 0.412715529 & -6.369654736 & -1.135856613 \\
$\mathrm{O}$ & 0.082627129 & -5.010673166 & -1.599413929 \\
$\mathrm{C}$ & 1.933036065 & -6.306386783 & -0.976057212 \\
$\mathrm{C}$ & 0.008222582 & -7.394166829 & -2.198319129 \\
$\mathrm{C}$ & -0.257251051 & -6.620487734 & 0.218715383 \\
$\mathrm{C}$ & -1.211375805 & -4.612657680 & -1.604618820 \\
$\mathrm{O}$ & -2.177650143 & -5.348049610 & -1.733006409 \\
$\mathrm{~N}$ & -1.306644102 & -3.240283643 & -1.482805331 \\
$\mathrm{O}$ & 1.407875340 & -1.687464066 & 0.095915079 \\
$\mathrm{C}$ & 2.251412346 & -1.676035347 & 1.287127969 \\
$\mathrm{C}$ & 0.318976773 & -2.525377774 & 0.006697323 \\
$\mathrm{C}$ & 1.459358965 & -1.161299240 & 2.494571621 \\
$\mathrm{C}$ & 2.836255028 & -3.071187028 & 1.542258659 \\
$\mathrm{C}$ & 3.363507486 & -0.691183086 & 0.911798089 \\
$\mathrm{H}$ & -0.092327681 & -3.221455013 & 0.934333014 \\
$\mathrm{H}$ & -1.336603761 & -6.734353422 & 0.108753637 \\
$\mathrm{~N}$ & -0.181207418 & -2.434785001 & -1.248887839 \\
$\mathrm{C}$ & -2.576332934 & -2.604751114 & -1.415803307 \\
$\mathrm{C}$ & -3.554393314 & -2.833814642 & -2.391315495 \\
$\mathrm{C}$ & -2.823330856 & -1.688052924 & -0.386163056 \\
$\mathrm{C}$ & -4.760451074 & -2.133746116 & -2.344236767 \\
$\mathrm{C}$ & -5.003998671 & -1.207323109 & -1.328387216 \\
$\mathrm{H}$ & -5.513193892 & -2.315809695 & -3.106467767 \\
$\mathrm{H}$ & -3.377227846 & -3.582648616 & -3.155835259 \\
$\mathrm{C}$ & -4.032926245 & -0.997425496 & -0.344490685 \\
$\mathrm{H}$ & -5.943667484 & -0.663451059 & -1.296222774 \\
$\mathrm{H}$ & -4.219897581 & -0.296573622 & 0.465315981 \\
$\mathrm{H}$ & -2.070899718 & -1.542511341 & 0.379292109 \\
\hline & 2.414362956 & -6.072427294 & -1.930680596 \\
\hline & -2.313339683 & -7.270711167 & -0.625281605 \\
\hline & -0.165814905 & 2.281328183 \\
\hline
\end{tabular}

\begin{tabular}{|c|c|c|c|}
\hline & & & \\
\hline & & & \\
\hline & & & \\
\hline & 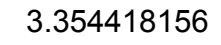 & & \\
\hline & & & 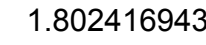 \\
\hline & & & \\
\hline & 3.9107 & -1.0 & ( \\
\hline & 35 & 06 & 0 \\
\hline & 6 & 3.744 & $-0 c \quad c$ \\
\hline & 5 & 16 & 176 \\
\hline & -0.906188632 & 99 & 912 \\
\hline & -1.1172 & 4.816 & -0.6 \\
\hline & -0.1 & 3.6 & 26 \\
\hline & -2.0 & 2.4 & -2 \\
\hline & -2.7 & & \\
\hline & -3.1 & & \\
\hline & -0.97 & & 0.3 \\
\hline & -1.68 & 3.3 & 1. \\
\hline & 33 & 3.3 & 218 \\
\hline & -1.8 & 34 & -2 \\
\hline & -1.2 & 38 & \\
\hline & -2.94 & 49 & -3 \\
\hline & -0.06 & 99 & -0.7 \\
\hline & -2 & & \\
\hline & 0.0 & 1.02 & 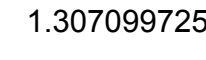 \\
\hline & -0.57 & 22 & -1.8 \\
\hline & -1.8862 & 0.32 & -3.5 \\
\hline & -2.6 & 0.5 & -1 \\
\hline & 1750 & .7358 & 0.000 \\
\hline
\end{tabular}

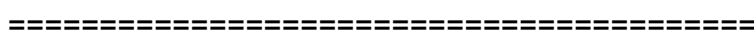

F5

$\begin{array}{cccc}======================================== \\ \mathrm{C} & 1.559156439 & -2.248158217 & -4.876264605 \\ \mathrm{C} & 0.195053754 & -2.937072016 & -4.761271455 \\ \mathrm{~N} & 2.075164118 & -1.900020435 & -3.544652577 \\ \mathrm{H} & 1.464156677 & -1.321401157 & -5.450922696 \\ \mathrm{H} & 2.248333271 & -2.908559605 & -5.427974021 \\ \mathrm{Cu} & 0.247270414 & -1.308112060 & -2.376589265 \\ \mathrm{~N} & -0.806073086 & -2.030460417 & -4.157649650 \\ \mathrm{H} & -1.553496077 & -2.593413844 & -3.760586209 \\ \mathrm{C} & -1.394215893 & -1.084986932 & -5.117666260\end{array}$




\begin{tabular}{|c|c|c|c|}
\hline & 5167 & 59 & \\
\hline & 0.277346131 & 3.808353639 & 107094507 \\
\hline & 7249820 & 4879 & 192 \\
\hline & 2.714905984 & -3.021537435 & -2 \\
\hline & 1.961465052 & -3.769118293 & -2.5 \\
\hline & 3.518 & -3.4 & 98 \\
\hline & $3.1163 c$ & -2.6 & 30 \\
\hline & -0.663 & -0.3 & 766 \\
\hline & -1.739478014 & -1.583337043 & -6.0 \\
\hline & -2.2 & -0.5 & \\
\hline & 0.5 & 2 & \\
\hline & 1.9 & & \\
\hline & 1.6 & & \\
\hline & 2.0 & & \\
\hline & 3.06 & & \\
\hline & & & \\
\hline & & & \\
\hline & & & \\
\hline & & & \\
\hline & 2 & 54 & \\
\hline & 1.6 & -6 & \\
\hline & -0.4 & -7 & 62 \\
\hline & -0.42 & -7.2 & \\
\hline & -1.4 & -4.7 & \\
\hline & -2.43 & -5.42 & \\
\hline & -1.404 & -3.4 & -1 \\
\hline & 1.58 & -2.6 & \\
\hline & 2.46 & $-3 . c$ & \\
\hline & 0.4 & -3.3 & \\
\hline & 2 & -2 & \\
\hline & & & \\
\hline & 3.620 & -2.02 & \\
\hline & 0.023753683 & -4.4 & \\
\hline & $-c$ & -2.8 & \\
\hline & -2.593 & -2.7 & \\
\hline & -3.67 & 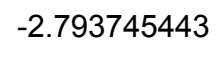 & \\
\hline & -2.6425338 & & \\
\hline & & & \\
\hline & & & \\
\hline & -5.588974932 & -1.979496759 & \\
\hline & -3.665192895 & -3.503837421 & \\
\hline & & & $5 /<0$ \\
\hline
\end{tabular}

\begin{tabular}{|c|c|c|c|}
\hline & 529 & 36 & \\
\hline & 7591266 & 372461720 & 089453928 \\
\hline & 17 & 75 & $056>-7$ \\
\hline & 1 & & -3.0 \\
\hline & 2.024 & -7.7 & -2.1 \\
\hline & 13 & -6.1 & -1. \\
\hline & 58 & -7.2 & -3 \\
\hline & 72 & -6.7 & -4 \\
\hline & 0.13 & -8.3 & -3.5 \\
\hline & -0.12 & -6 & \\
\hline & 1.5 & -7 & \\
\hline & -0.0 & -8.3 & \\
\hline & 1.3 & & \\
\hline & & & \\
\hline & & & \\
\hline & & & \\
\hline & & & \\
\hline & & & \\
\hline & & & \\
\hline & & & \\
\hline & & & \\
\hline & 7 & & \\
\hline & 3 & & \\
\hline & 0 & & \\
\hline & 1.9 & & -1 \\
\hline & 7 & & \\
\hline & 33 & & -1.3 \\
\hline & 9 & 0 & \\
\hline & .5 & & \\
\hline & 9 & $-C$ & \\
\hline & & & \\
\hline & 0.3022 & & \\
\hline & .0 & & \\
\hline & 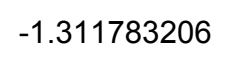 & & -3. \\
\hline & 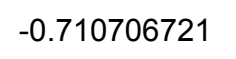 & & -1.3 \\
\hline & & & \\
\hline & & & \\
\hline & & & \\
\hline & & & \\
\hline & & & \\
\hline & & & \\
\hline & & & \\
\hline
\end{tabular}




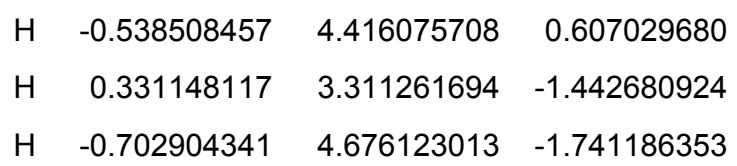

\section{F5-TS
F=}

\begin{tabular}{|c|c|c|c|}
\hline $\mathrm{C}$ & 1.924410673 & -2.717963542 & -4.71 \\
\hline C & 79006116 & -3.230349815 & 46828170 \\
\hline$N$ & 2.287185729 & -2.072025984 & -3.4 \\
\hline $\mathrm{H}$ & 2.036757502 & 4344 & \\
\hline $\mathrm{H}$ & 2.596097536 & -3.557784093 & -4.9 \\
\hline $\mathrm{Cu}$ & 0.465221310 & -1.161787926 & -2.605 \\
\hline 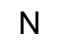 & -0.467 & -2.1 & -4 \\
\hline $\mathrm{H}$ & 593 & -2.5 & -6 \\
\hline 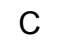 & -0.6 & -1 & -5.604951078 \\
\hline 1 & 0.262737430 & -3.765 & 6. \\
\hline 4 & 2 & 1 & -3.8 \\
\hline $\mathrm{H}$ & 23523 & 590 & 年 \\
\hline C & 2.833978370 & -2.993172903 & \\
\hline $\mathrm{H}$ & 206 & -3.6 & -2.1 \\
\hline $\mathrm{H}$ & 3.709802534 & -3.548479993 & -2.80655950 \\
\hline $\mathrm{H}$ & 7 & -2 & -1 \\
\hline $\mathrm{H}$ & 5 & -0.6 & -3. \\
\hline $\mathrm{H}$ & -0.888990142 & -1.7 & -6 . \\
\hline $\mathrm{H}$ & 2 & -0 & -5.39584365 \\
\hline $\mathrm{O}$ & 0.0001 & 0.75 & 0.700 \\
\hline S & 01 & 1.5 & -0.4 \\
\hline $\mathrm{C}$ & 4 & & -5 \\
\hline $\mathrm{O}$ & 27 & & -2 \\
\hline $\mathrm{O}$ & 504 & 0.80 & -2 \\
\hline$F$ & 3.53984 & 2.3 & -5 \\
\hline$F$ & 1.439846512 & 2.092851436 & 55837 \\
\hline$F$ & 2.005000290 & & -0.00 \\
\hline C & 0.367 & 56 & -1.18 \\
\hline $\mathrm{O}$ & 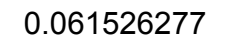 & -6 & -1 \\
\hline $\mathrm{C}$ & 2 & - & -0.99896120 \\
\hline C & -0.020049211 & -7.3 & -2 \\
\hline $\mathrm{C}$ & -0.339 & -6.6 & 0 \\
\hline$C$ & -1.228803022 & -4.5 & -1.6 \\
\hline $\mathrm{O}$ & -2.198936815 & -5.304605684 & -1.826 \\
\hline $\mathrm{N}$ & -1.317763269 & -3.211905582 & -1 \\
\hline
\end{tabular}

\begin{tabular}{|c|c|c|c|}
\hline & 9722811 & -1.692356923 & \\
\hline & 161517215 & 1845 & $1.39352053 c$ \\
\hline & 0.275241317 & -2.550975082 & 10371692 \\
\hline & 1.333635954 & -1.350256634 & 201 \\
\hline & 315715572 & 3859 & .550323 \\
\hline & 4 & -0 & \\
\hline & 142384350 & 2603 & \\
\hline & -0.199543408 & 2253 & \\
\hline & -2.587981199 & -2.576581126 & 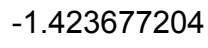 \\
\hline & 496 & -2.7 & \\
\hline & -2.852430353 & -1.730468157 & 01411 \\
\hline & -4.744108297 & -2.020160847 & -2 \\
\hline & -4.9 & -1. & \\
\hline & -5.4 & -2 . & \\
\hline & -3.358400084 & -3.432 & \\
\hline & -4.0531 & 03 & . \\
\hline & -5.9 & 9 & \\
\hline & 3096002 & -0.376982760 & \\
\hline & 34755 & 3487 & \\
\hline & 8426 & 1525 & \\
\hline & 2.24 & -7.2 & \\
\hline & 690 & -5 & \\
\hline & 5 & -1.04000 & 2 \\
\hline & 0.479172778 & 9 & \\
\hline & 294123440 & 1582 & \\
\hline & & & \\
\hline & -1 & 37 & \\
\hline & 36981 & 265 & \\
\hline & 0.835316738 & -0.388463172 & \\
\hline & 1.991022019 & -1.253105322 & 0.4000 \\
\hline & ז & -2.1004000 & . \\
\hline & .0002 & -3 & \\
\hline & 3.387444533 & & \\
\hline & & & \\
\hline & 3.936040676 & - & \\
\hline & & & \\
\hline & & & \\
\hline & 0.013 & & \\
\hline & -0.5 & & \\
\hline & -1.8535346 & 780101 & -2 \\
\hline & 1 & & \\
\hline & 3 & 1.438230706 & 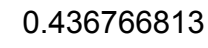 \\
\hline
\end{tabular}




$\begin{array}{llll}\text { H } & -2.572903437 & 0.670547706 & -1.752756779 \\ \text { H } & -2.069806941 & -0.180288751 & -3.173626557 \\ \text { C } & -2.029942092 & 1.985836582 & -3.437277261 \\ \text { H } & -0.123173303 & 1.309077221 & 1.299828593 \\ \text { H } & -1.629612297 & 0.760080621 & 0.593848834 \\ \text { C } & -1.297736880 & 2.900302989 & 0.407051951 \\ \text { H } & -3.085316248 & 2.035938521 & -3.737122859 \\ \text { H } & -1.443357097 & 1.844036663 & -4.350715945 \\ \text { C } & -1.597555638 & 3.311135969 & -2.790047844 \\ \text { H } & -0.510568141 & 3.557271095 & 0.017298157 \\ \text { H } & -1.444865994 & 3.196111805 & 1.452704369 \\ \text { C } & -2.625405293 & 3.128332541 & -0.363664969 \\ \text { C } & -2.516558870 & 3.887416113 & -1.700455785 \\ \text { H } & -0.575284952 & 3.214880396 & -2.413298556 \\ \text { H } & -1.536555995 & 4.056545831 & -3.593101947 \\ \text { H } & -3.299618496 & 3.710892289 & 0.276355436 \\ \text { H } & -3.137903630 & 2.169906025 & -0.509553692 \\ \text { H } & -3.529494869 & 4.004707136 & -2.111353419 \\ \text { H } & -2.163881129 & 4.903887274 & -1.477056442\end{array}$

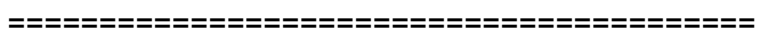

G1

\begin{tabular}{|c|c|c|c|}
\hline C & 0.316763986 & -2.346395370 & -4.278958893 \\
\hline C & -1.055838687 & -2.533370592 & -3.635698629 \\
\hline $\mathrm{N}$ & 1.330925002 & -2.143057062 & -3.223562534 \\
\hline $\mathrm{H}$ & 0.322316332 & -1.454971902 & -4.905236446 \\
\hline $\mathrm{H}$ & 0.565245232 & -3.212336240 & -4.908442582 \\
\hline $\mathrm{Cu}$ & 0.458901539 & -0.781177005 & -1.876870856 \\
\hline $\mathrm{N}$ & -1.357886969 & -1.356375580 & -2.791294530 \\
\hline $\mathrm{H}$ & -1.966389425 & -1.647253800 & -2.029994258 \\
\hline C & -1.977650394 & -0.225469143 & -3.509426041 \\
\hline $\mathrm{H}$ & -1.825222543 & -2.687919111 & -4.404864481 \\
\hline $\mathrm{H}$ & -1.054586029 & -3.412365399 & -2.982611325 \\
\hline $\mathrm{H}$ & 2.125442797 & -1.604729620 & -3.599687877 \\
\hline C & 1.813619325 & -3.393659476 & -2.619037526 \\
\hline $\mathrm{H}$ & 1.035372702 & -3.851058261 & -2.000413896 \\
\hline $\mathrm{H}$ & 2.129216482 & -4.114229009 & -3.385472818 \\
\hline $\mathrm{H}$ & 2.668782087 & -3.165758568 & -1.984106626 \\
\hline $\mathrm{H}$ & -1.282909184 & 0.145526134 & -4.262060930 \\
\hline $\mathrm{H}$ & -2.927654911 & -0.514165313 & -3.9796476 \\
\hline $\mathrm{H}$ & -2.143422305 & 0.581019489 & -2.7935232 \\
\hline
\end{tabular}

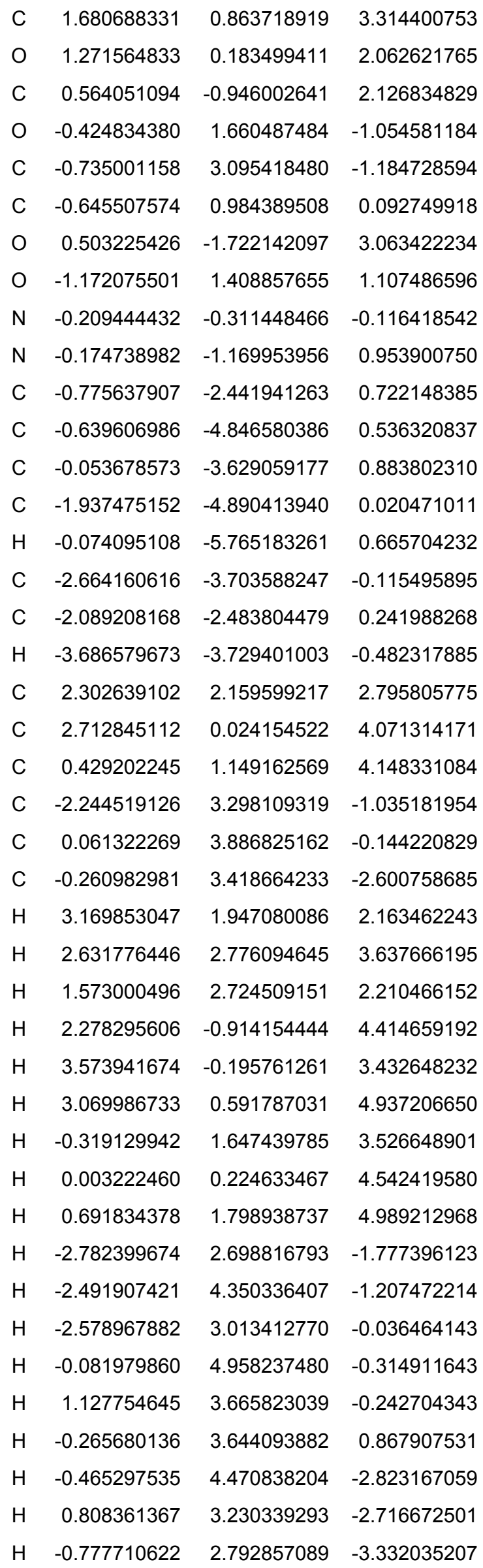




$\begin{array}{lrrr}\text { H } & 0.947201969 & -3.593875274 & 1.297409254 \\ \text { H } & -2.644736522 & -1.553196133 & 0.169009473 \\ \text { H } & -2.385507767 & -5.841150804 & -0.252262372 \\ \text { O } & 0.907128932 & 0.701049409 & -3.850896350 \\ \text { S } & 2.306110231 & 0.956547643 & -4.319703271 \\ \text { C } & 2.092367231 & 0.930941731 & -6.166880679 \\ \text { O } & 2.833991978 & 2.292012088 & -4.005218014 \\ \text { O } & 3.221394656 & -0.190339317 & -4.063270408 \\ \text { F } & 3.263113846 & 1.090796424 & -6.789708778 \\ \text { F } & 1.253326988 & 1.897871806 & -6.559809953 \\ \text { F } & 1.570675118 & -0.258147923 & -6.546813701 \\ \text { C } & 4.138800574 & 0.687286334 & -0.558681132 \\ \text { C } & 4.115951191 & -0.661917275 & 0.210628373 \\ \text { C } & 2.797420877 & -1.275445338 & -0.226233088 \\ \text { H } & 4.098192430 & -0.510559571 & 1.299393138 \\ \text { H } & 4.982227310 & -1.294795965 & -0.013844723 \\ \text { C } & 2.649690623 & 1.022511648 & -0.840214484 \\ \text { H } & 4.642223637 & 0.552418661 & -1.519999731 \\ \text { H } & 4.648371148 & 1.485077276 & -0.009471573 \\ \text { C } & 2.072061147 & -0.365114308 & -0.872222005 \\ \text { H } & 2.186018692 & 1.557753765 & -0.005327612 \\ \text { H } & 2.509211472 & 1.612784473 & -1.741940464 \\ \text { H } & 2.492349515 & -2.283807386 & 0.025297935\end{array}$

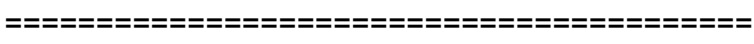

\section{G1-TS}

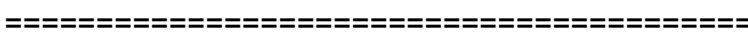

$\begin{array}{lrrr}\mathrm{C} & 0.172092546 & -2.380363100 & -3.925490942 \\ \mathrm{C} & -0.883894952 & -2.439734520 & -2.820832031 \\ \mathrm{~N} & 1.524643798 & -2.388720952 & -3.332745351 \\ \mathrm{H} & 0.078048698 & -1.450388442 & -4.488593673 \\ \mathrm{H} & 0.033831865 & -3.216317230 & -4.626125708 \\ \mathrm{Cu} & 1.412987813 & -1.022487216 & -1.709625487 \\ \mathrm{~N} & -0.698764063 & -1.299512143 & -1.898649112 \\ \mathrm{H} & -1.034814370 & -1.565760484 & -0.976717422 \\ \mathrm{C} & -1.369316539 & -0.055898948 & -2.324264435 \\ \mathrm{H} & -1.892289325 & -2.448731092 & -3.258854897 \\ \mathrm{H} & -0.766019167 & -3.357111396 & -2.234480100 \\ \mathrm{H} & 2.155057004 & -1.918254491 & -3.992594913 \\ \mathrm{C} & 2.033171890 & -3.725865716 & -3.003264434 \\ \mathrm{H} & 1.448432132 & -4.166483860 & -2.189899918 \\ \mathrm{H} & 2.006011368 & -4.402157476 & -3.869219887\end{array}$

H $3.066817906 \quad-3.631603785 \quad-2.665582921$

$\begin{array}{llll}\mathrm{H} & -0.982589570 & 0.249469077 & -3.295650308\end{array}$

H $\quad-2.460301829 \quad-0.177568097 \quad-2.377179308$

H $\quad-1.114126526 \quad 0.731544781 \quad-1.613212780$

$\begin{array}{llll}\text { C } & 4.576022397 & 0.109485698 & 2.862731444\end{array}$

$\begin{array}{llll}\text { O } & 3.718390841 & -0.434657070 & 1.787369627\end{array}$

C $\quad 2.944262822 \quad-1.498439322 \quad 2.028158461$

O $1.249909354 \quad 1.476769364 \quad-0.460165049$

C $\quad \begin{array}{llll}1.042655118 & 2.933174240 & -0.359109141\end{array}$

$\begin{array}{llll}\text { C } & 1.345242955 & 0.692269283 & 0.625035440\end{array}$

O $3.099530508 \quad-2.340767680 \quad 2.893332056$

$\begin{array}{llll}\text { O } & 1.186964573 & 1.017868105 & 1.789906209\end{array}$

$\begin{array}{llll}\mathrm{N} & 1.609016264 & -0.601136639 & 0.188029998\end{array}$

N $\quad \begin{array}{lll}1.862424882 & -1.554351528 & 1.142171513\end{array}$

C $\quad 0.982945876 \quad-2.675723641 \quad 1.130871417$

$\begin{array}{llll}\text { C } & 0.567613899 & -5.033466234 & 0.830412650\end{array}$

$\begin{array}{llll}\text { C } & 1.467533197 & -3.974647410 & 0.959779334\end{array}$

$\begin{array}{llll}\text { C } & -0.810130686 & -4.802545052 & 0.866379646\end{array}$

H $\quad 0.946577122 \quad-6.042408118 \quad 0.694199538$

$\begin{array}{llll}\text { C } & -1.288099899 & -3.503389872 & 1.066567948\end{array}$

$\begin{array}{llll}\text { C } & -0.394011798 & -2.442447953 & 1.208067752\end{array}$

$\begin{array}{llll}\text { H } & -2.356991577 & -3.318607398 & 1.129498583\end{array}$

$\begin{array}{llll}\text { C } & 5.107780500 & 1.401742127 & 2.242837884\end{array}$

$\begin{array}{llll}\text { C } & 5.717120415 & -0.863494627 & 3.169361544\end{array}$

C $\quad 3.708586821 \quad 0.405852805 \quad 4.088396749$

$\begin{array}{llll}\text { C } & -0.319831562 & 3.210286243 & 0.280608709\end{array}$

C $\quad 2.189143570 \quad 3.558959835 \quad 0.439766163$

$\begin{array}{lllll}\text { C } & 1.079553877 & 3.384053956 & -1.819519803\end{array}$

H $5.675998945 \quad 1.189308386 \quad 1.332275824$

H $\quad 5.767873066 \quad 1.910087430 \quad 2.952112591$

H $4.282306166 \quad 2.072084166 \quad 1.990443338$

H $\quad 5.331725745 \quad-1.798264509 \quad 3.576219438$

H $\quad 6.289779028 \quad-1.079973445 \quad 2.261812849$

H $6.395523480 \quad-0.407826707 \quad 3.898431455$

H $2.844494148 \quad 1.005306744 \quad 3.790048524$

H $3.355226655 \quad-0.517850626 \quad 4.549975764$

H $4.296922202 \quad 0.962423470 \quad 4.824813582$

H $\quad-1.117480329 \quad 2.738238750 \quad-0.302228108$

H $\quad-0.504286781 \quad 4.289233035 \quad 0.292936207$

H $\quad-0.353543579 \quad 2.833221535 \quad 1.303762327$

H $2.100312802 \quad 4.649424259 \quad 0.410093541$

$\begin{array}{llll}\text { H } & 3.149457309 & 3.285467012 & -0.006790637\end{array}$ 


$\begin{array}{lrrr}\text { H } & 2.169038531 & 3.230582522 & 1.480064621 \\ \text { H } & 0.908638817 & 4.464090162 & -1.872984130 \\ \text { H } & 2.041965027 & 3.152267086 & -2.281246143 \\ \text { H } & 0.312856085 & 2.871705474 & -2.403994451 \\ \text { H } & 2.537882609 & -4.145530288 & 0.941251193 \\ \text { H } & -0.738416479 & -1.427851724 & 1.386710662 \\ \text { H } & -1.505144571 & -5.629678438 & 0.757953452 \\ \text { O } & 1.380829145 & 0.569488988 & -3.291142661 \\ \text { S } & 2.254087127 & 0.786999493 & -4.489552849 \\ \text { C } & 1.008798086 & 1.501331163 & -5.670865052 \\ \text { O } & 3.278161226 & 1.829488478 & -4.329730725 \\ \text { O } & 2.687327281 & -0.481635542 & -5.129282732 \\ \text { F } & 1.521270813 & 1.651756475 & -6.894151370 \\ \text { F } & 0.576484077 & 2.690346200 & -5.227585275 \\ \text { F } & -0.062068917 & 0.675960684 & -5.757158062 \\ \text { C } & 5.438445471 & 0.062701443 & -1.666407687 \\ \text { C } & 5.542610049 & -1.354877481 & -1.039131766 \\ \text { C } & 4.093294149 & -1.800030496 & -0.961293066 \\ \text { H } & 5.952764558 & -1.317022835 & -0.019078137 \\ \text { H } & 6.178795869 & -2.032622540 & -1.619471188 \\ \text { C } & 4.006702210 & 0.558334386 & -1.328675585 \\ \text { H } & 5.533621458 & -0.004971557 & -2.754125374 \\ \text { H } & 6.210542805 & 0.747440187 & -1.301909181 \\ \text { C } & 3.292855114 & -0.758814089 & -1.199453621 \\ \text { H } & 3.970899985 & 1.060312876 & -0.357208828 \\ \text { H } & 3.597230631 & 1.225696837 & -2.081636700 \\ \text { H } & 3.780634776 & -2.793604742 & -0.663227561\end{array}$

\begin{tabular}{|c|c|c|c|}
\hline \multicolumn{4}{|l|}{$\begin{array}{l}\text { G5 } \\
===\end{array}$} \\
\hline C & -0.750522282 & -0.651599965 & -3.252319957 \\
\hline C & -1.378223268 & 0.127771112 & -2.099372529 \\
\hline$N$ & -0.369125473 & -2.015159057 & -2.795250237 \\
\hline $\mathrm{H}$ & 0.164203512 & -0.162716236 & -3.586264261 \\
\hline $\mathrm{H}$ & -1.436877739 & -0.708322284 & -4.107813712 \\
\hline $\mathrm{Cu}$ & 0.567764966 & -1.741298528 & -0.982847328 \\
\hline$N$ & -0.411023868 & 0.183430290 & -0.987607430 \\
\hline $\mathrm{H}$ & -0.898108757 & 0.186085791 & -0.095783286 \\
\hline C & 0.520144685 & 1.325701837 & -1.044243713 \\
\hline $\mathrm{H}$ & -1.676460996 & 1.130389672 & -2.436713470 \\
\hline $\mathrm{H}$ & -2.279913399 & -0.382287348 & -1.743001977 \\
\hline
\end{tabular}

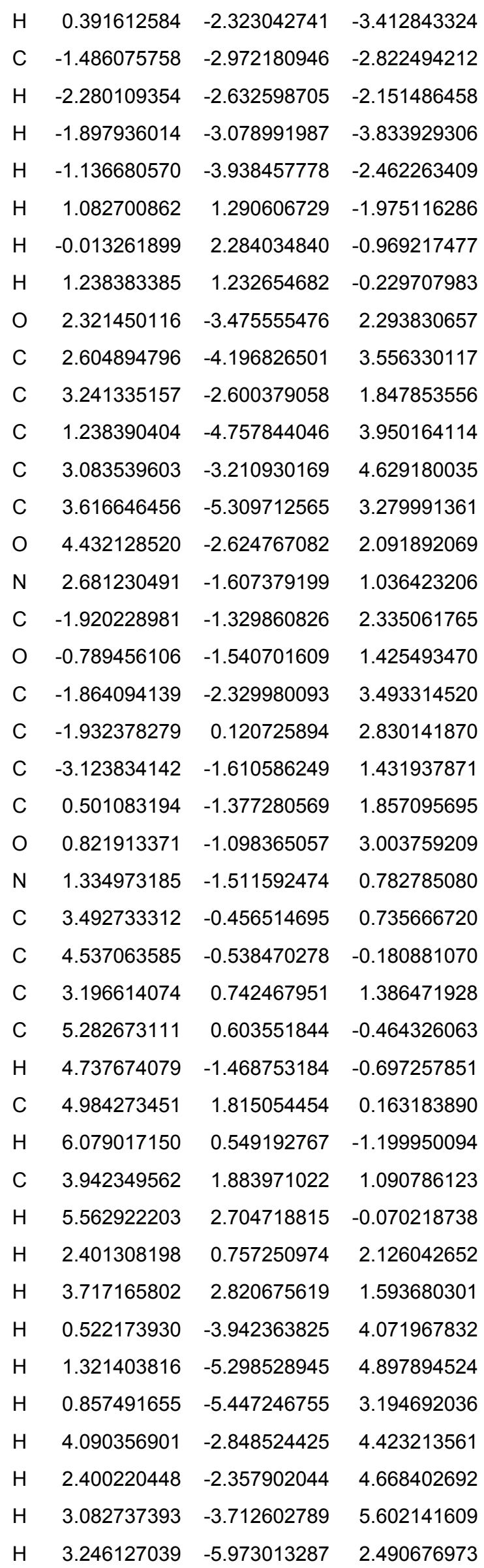




\begin{tabular}{|c|c|c|c|}
\hline & 87 & & \\
\hline & 3.769677248 & -5.906024469 & .1860227 \\
\hline & .762347089 & -3.348205002 & .1052694 \\
\hline & 2.788695015 & -2.27179 & 635 \\
\hline & -1.017883220 & -2.1 & 92 \\
\hline & -2.827704457 & 53 & 89 \\
\hline & -1.955245830 & 0.81800 & 32 \\
\hline & -1.04 & 30 & 60 \\
\hline & -4.0 & 72 & 1.9909 \\
\hline & $-3.13 s$ & -0.9 & \\
\hline & -3.0 & 4 & \\
\hline & 0.9 & 5 & \\
\hline & 0.0 & & \\
\hline & 2.37 & & \\
\hline & 9 & & \\
\hline & 0.0 & & \\
\hline & 2. & & \\
\hline & 3.03 & -3 & 0 \\
\hline & 2.550 & -4.5 & \\
\hline & -0.78 & -5.8 & 1. \\
\hline & 0.96 & -5.7 & 73 \\
\hline & 0.09 & -6.9 & -0. \\
\hline & 3.572 & -4.9 & -2.5 \\
\hline & 2.51 & -3.7 & -3 \\
\hline & 1.54 & -5.5 & -2 \\
\hline & -0.59 & -6.7 & \\
\hline & -0.3 & & \\
\hline & 1.4 & & \\
\hline & 1.6 & -8 & \\
\hline & & & \\
\hline & 1.733 & & \\
\hline & 2. & & \\
\hline & 1. & & -3.06 \\
\hline & 0.52 & -5.2 & \\
\hline & 1.61 & & \\
\hline & & & \\
\hline & & & \\
\hline & & & \\
\hline & & & 0.2 \\
\hline & & -1.759589953 & \\
\hline & & & 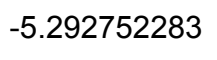 \\
\hline & 4.09635 & 1.241276 & 70344 \\
\hline
\end{tabular}

F $\quad 2.107380042 \quad 1.218819463 \quad-4.160318946$

\begin{tabular}{|c|c|c|c|}
\hline \multicolumn{4}{|c|}{ G5-TS } \\
\hline C & -0.842003746 & 3 & -3.0633351 \\
\hline 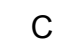 & 72444876 & 253998345 & -1.8593142 \\
\hline v & -0 & -1.995105094 & \\
\hline + & 0 & 9 & -3.6006876 \\
\hline 1 & -1.691621908 & -0.648219583 & -3.7609685 \\
\hline $\mathrm{Cu}$ & 0.811740096 & -1.726856634 & $-0 c$ \\
\hline & -0.01 & 20 & -0.9 \\
\hline & -0.34 & 0.44 & 0005 \\
\hline & 5 & 1.33 & -1 \\
\hline & -1.473660451 & 1.25 & -2.2 \\
\hline$H$ & -2.016348288 & -0.174 & -1.3 \\
\hline-1 & 0.137953983 & -2.372456540 & -3.3 \\
\hline ¿ & -1.630383844 & -2.878382441 & -2 \\
\hline-1 & -2.269962276 & -2.488881656 & \\
\hline 1 & -2.231499419 & -2.9 & -3 \\
\hline 7 & -1.278 & -3.86 & \\
\hline H & 1.42 & 1.1 & \\
\hline-1 & 8 & 5 & \\
\hline-1 & & & \\
\hline 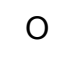 & 5 & & \\
\hline & & & \\
\hline & & & \\
\hline c & 8 & & \\
\hline c & 2 & 8 & \\
\hline 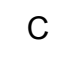 & - & 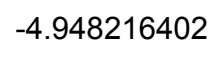 & \\
\hline ○ & 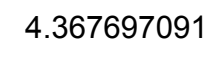 & & \\
\hline v & 8 & 8 & \\
\hline$c$ & -1.85 & -1.4 & 2271 \\
\hline O & -0.68 & 3 & 13023 \\
\hline c & -1.7 & -2 & \\
\hline C & -1.96 & -0.0 & 2824 \\
\hline C & -3.0 & -1 & \\
\hline 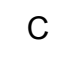 & 5 & -1.4 & 1.88 \\
\hline 0 & 7 & -1.0 & 3.01 \\
\hline IV & 3 & -1.765586020 & 0.9032 \\
\hline C & 3.502650582 & -0.479324498 & 0.86 \\
\hline c & 875 & 81687 & \\
\hline
\end{tabular}




\begin{tabular}{|c|c|c|c|}
\hline & 5 & & \\
\hline & 820776212 & .702747767 & 76713257 \\
\hline & 290914123 & 9951 & 3984 \\
\hline & .700942864 & 1.867502217 & 8173 \\
\hline & 5.353451599 & 0.712717136 & $-1.7^{\prime}$ \\
\hline & 4 & 02 & 627 \\
\hline & 5.1621 & 53 & 270 \\
\hline & 2.80 & 74 & 057 \\
\hline & 3.900125428 & 2.760 & $1.7 \varepsilon$ \\
\hline & 0.5585 & -4.5 & \\
\hline & $1.4 \mathrm{C}$ & -5.7 & \\
\hline & 1.5 & -5 & \\
\hline & 3.50 & -2.5 & \\
\hline & 4 & -2 & \\
\hline & 2.5 & -3 & \\
\hline & 7.00 & -5.5 & \\
\hline & 1.00 & & \\
\hline & 7 & & \\
\hline & 3 & -3 & \\
\hline & 2 & -2 & \\
\hline & -0.9 & -2.2 & \\
\hline & $-2.8 s$ & 0.0 & \\
\hline & -2.00 & 0.6 & \\
\hline & -1.11 & 79 & \\
\hline & -3.9688 & -1.7 & 381 \\
\hline & -3.05 & -1.0 & 500 \\
\hline & -2.925 & -2.76 & 241 \\
\hline & 1.19 & -3.6 & \\
\hline & 0.04 & -4.2 & \\
\hline & 2.55 & -4.1 & \\
\hline & -0.8 & -3 & \\
\hline & -0 . & -5 & \\
\hline & ; & -5.0 & \\
\hline & 3. & -3.50 & 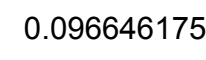 \\
\hline & ; & -4.43 & 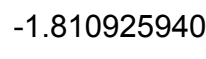 \\
\hline & -1.104 & -5.84 & \\
\hline & 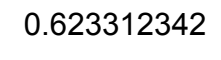 & -6.10 & \\
\hline & -0 & & \\
\hline & & & \\
\hline & 3 & -3.511998397 & $2.100+0010$ \\
\hline & & -4.925874151 & - \\
\hline & & & \\
\hline
\end{tabular}
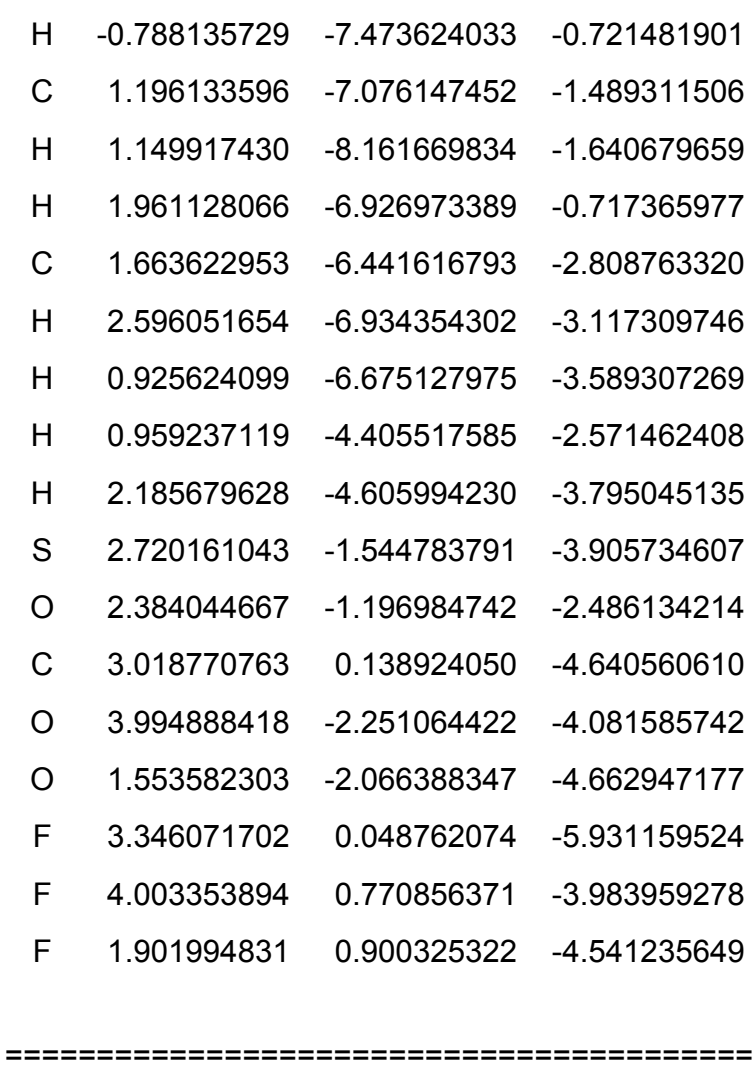

H

\begin{tabular}{crrr}
\hline$====================================$ \\
$\mathrm{C}$ & 0.505198407 & -2.651077091 & -3.215861202 \\
$\mathrm{C}$ & 0.157717968 & -2.657914496 & -1.717231565 \\
$\mathrm{~N}$ & 1.932572092 & -2.299723905 & -3.436146405 \\
$\mathrm{H}$ & -0.095257823 & -1.891044465 & -3.724373205 \\
$\mathrm{H}$ & 0.264418179 & -3.626719211 & -3.661734018 \\
$\mathrm{Cu}$ & 2.377065103 & -0.781300067 & -2.124900748 \\
$\mathrm{~N}$ & 0.487558096 & -1.366574288 & -1.116862345 \\
$\mathrm{H}$ & 0.758634240 & -1.447000018 & -0.141101802 \\
$\mathrm{C}$ & -0.532564022 & -0.319048405 & -1.249484148 \\
$\mathrm{H}$ & -0.904507632 & -2.925660057 & -1.604358979 \\
$\mathrm{H}$ & 0.742434401 & -3.431040911 & -1.208761250 \\
$\mathrm{H}$ & 2.046268315 & -2.009265412 & -4.404506252 \\
$\mathrm{C}$ & 2.863713508 & -3.422977687 & -3.176791507 \\
$\mathrm{H}$ & 2.871570489 & -3.651135378 & -2.109391903 \\
$\mathrm{H}$ & 2.584093500 & -4.327309121 & -3.734983606 \\
$\mathrm{H}$ & 3.872424373 & -3.115676933 & -3.457994941 \\
$\mathrm{H}$ & -0.645405723 & -0.034659225 & -2.300628459 \\
$\mathrm{H}$ & -1.515564473 & -0.633036789 & -0.867059504 \\
$\mathrm{H}$ & -0.182608816 & 0.550525618 & -0.691928103 \\
$\mathrm{O}$ & 3.459666306 & 0.464718420 & -1.116592410 \\
$\mathrm{~S}$ & 3.231701195 & 0.530601977 & 0.391243224
\end{tabular}




\section{C $\quad 3.909962138 \quad-1.124836993 \quad 0.932622168$ \\ $\begin{array}{llll}\text { O } & 4.067983998 & 1.531468295 & 1.046615437\end{array}$ \\ $\begin{array}{llll}\text { O } & 1.807768704 & 0.443452071 & 0.775755421\end{array}$ \\ F $\quad 3.825303344 \quad-1.266610596 \quad 2.256696354$ \\ F $\quad \begin{array}{llll}5.181050018 & -1.283427454 & 0.554115617\end{array}$ \\ $\begin{array}{llll}F & 3.181096360 & -2.131572977 & 0.363910079\end{array}$}

\begin{tabular}{|c|c|c|c|}
\hline \\
\hline C & 0.534480372 & 0.993072345 & -0.599649749 \\
\hline & 0.244590593 & .403532698 & 0.02 \\
\hline & 0.094534976 & -0.094944395 & 1.517 \\
\hline C & -0.006865891 & 214272386 & 1.677045337 \\
\hline & 0.031876544 & 2.052619993 & 8503 \\
\hline $\mathrm{H}$ & 0.068034574 & 1.118133308 & -1.58 \\
\hline & 1.046682103 & -1.121857331 & -0.178143162 \\
\hline & -0.683192114 & -0.842800349 & -0.364196610 \\
\hline & 0.040700379 & -0.857547860 & 2.287582245 \\
\hline & 0.697067454 & 2.919595965 & 0.507736300 \\
\hline & -0.968709071 & 2.433438308 & 0.191630531 \\
\hline & & & \\
\hline
\end{tabular}

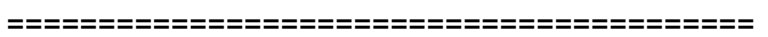

12

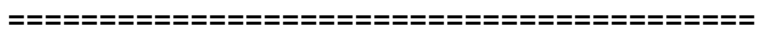

C $\quad 0.074776749 \quad-0.809485549 \quad-0.588939944$

C $\quad-0.964182222 \quad-0.799837505 \quad 0.543142079$

C $\quad 1.021263260 \quad 0.401616660 \quad-0.503762998$

C $\quad-1.869246731 \quad 0.460197518 \quad 0.454172771$

C $\quad 0.247725339 \quad 1.688952726 \quad-0.229209477$

C $\quad-1.007718656 \quad 1.633104025 \quad 0.169678750$

H $\quad-0.450554166 \quad-0.786934847 \quad-1.552990644$

H $\quad 0.653620652 \quad-1.739760215 \quad-0.564363831$

H $\quad-0.445580302 \quad-0.795264955 \quad 1.510155017$

H $\quad-1.583226139-1.704424830 \quad 0.512995575$

H $\quad 1.593771004 \quad 0.501449288 \quad-1.435114701$

H $\quad 1.763792944 \quad 0.250374093 \quad 0.294368856$

$\begin{array}{llll}H & -2.429509741 & 0.592038819 & 1.388671684\end{array}$

H $\quad-2.618763341 \quad 0.339329263 \quad-0.340833076$

H $\quad 0.769109647 \quad 2.636249377 \quad-0.368130173$

13

$\begin{array}{rrrr}========================================= \\ \text { C } & -3.858711120 & 0.228731697 & -4.896777893 \\ \text { C } & -3.087461950 & 1.312128821 & -5.675800214 \\ \text { C } & -3.016053968 & -0.904505218 & -4.274415155 \\ \text { C } & -1.749553937 & 1.750775261 & -5.027728170 \\ \text { C } & -1.925948695 & -1.509204597 & -5.177429256 \\ \text { C } & -0.718777913 & 0.697068916 & -5.182160121 \\ \text { C } & -0.694811605 & -0.612874592 & -5.320103096 \\ \text { H } & -4.428434195 & 0.704039323 & -4.087545758 \\ \text { H } & -4.603961458 & -0.206958013 & -5.575185638 \\ \text { H } & -3.735494628 & 2.188626373 & -5.801901769 \\ \text { H } & -2.857098412 & 0.949436997 & -6.684155408 \\ \text { H } & -3.701845898 & -1.698413848 & -3.953819145 \\ \text { H } & -2.527104257 & -0.538150078 & -3.363323794 \\ \text { H } & -1.894058146 & 2.003523445 & -3.967075858 \\ \text { H } & -1.404775138 & 2.674991669 & -5.511146392 \\ \text { H } & -2.338347598 & -1.744216163 & -6.170459175 \\ \text { H } & -1.600752039 & -2.468191215 & -4.754847419 \\ \text { H } & 0.251054507 & -1.121441710 & -5.515233251\end{array}$

14

$\begin{array}{cccc}==================================== \\ \text { C } & -3.687818335 & -1.197560875 & -2.441592704 \\ \text { C } & -2.601270641 & -1.473942814 & -1.368921338 \\ \text { C } & -4.909191227 & -0.380830288 & -1.980824518 \\ \text { C } & -1.305962528 & -0.644147819 & -1.488254199 \\ \text { C } & -4.580711933 & 0.956761229 & -1.281407864 \\ \text { C } & -1.402090550 & 0.842785113 & -1.072034854 \\ \text { C } & -3.595650905 & 1.810643367 & -2.067509327 \\ \text { C } & -2.294419428 & 1.666454571 & -1.920339362 \\ \text { H } & -4.063654002 & -2.153529528 & -2.825933852 \\ \text { H } & -3.224035899 & -0.692330710 & -3.295960596 \\ \text { H } & -2.307892344 & -2.528502673 & -1.433341450 \\ \text { H } & -3.021215816 & -1.349902505 & -0.360906316 \\ \text { H } & -5.513313041 & -0.985150096 & -1.290314001 \\ \text { H } & -5.543357542 & -0.188801116 & -2.856360709 \\ \text { H } & -0.938053623 & -0.700929414 & -2.520766539 \\ \text { H } & -0.535568962 & -1.110340493 & -0.860258806 \\ \text { H } & -4.156893389 & 0.746626204 & -0.291529175 \\ \text { H } & -5.509794507 & 1.513625425 & -1.109691442 \\ \text { H } & -0.390676700 & 1.270826011 & -1.067759362 \\ \text { H } & -1.753511310 & 0.901402652 & -0.027418622\end{array}$


15

$\begin{array}{crrr}\text { ===================================== } \\ \text { C } & -2.061991198 & 0.853296952 & -0.036310870 \\ \mathrm{C} & -1.870223813 & 0.545145313 & 1.229808741 \\ \mathrm{H} & -2.702365105 & 0.128360810 & 1.801157795 \\ \mathrm{C} & -1.291672330 & 1.302852492 & -1.209867870 \\ \mathrm{H} & -0.408429710 & 1.864816341 & -0.865629026 \\ \mathrm{H} & -1.877832105 & 2.011648356 & -1.810142776 \\ \mathrm{C} & -0.557171166 & 0.704504780 & 2.000760565 \\ \mathrm{H} & -0.786640474 & 0.616730746 & 3.068877175 \\ \mathrm{H} & -0.164803790 & 1.719659659 & 1.857202508 \\ \mathrm{C} & -0.855668418 & 0.122975285 & -2.121217246 \\ \mathrm{H} & -0.154896981 & 0.507270907 & -2.874149564 \\ \mathrm{H} & -1.742439807 & -0.218413172 & -2.669010101 \\ \mathrm{C} & 0.560176644 & -0.309154257 & 1.648541683 \\ \mathrm{H} & 0.129706169 & -1.316354677 & 1.582771547 \\ \mathrm{H} & 1.251161241 & -0.335594859 & 2.499818495 \\ \mathrm{C} & 1.373393516 & 0.013590793 & 0.366392535 \\ \mathrm{H} & 2.441523923 & -0.017025478 & 0.614920562 \\ \mathrm{H} & 1.185491051 & 1.049342181 & 0.063569156 \\ \mathrm{C} & 1.173438931 & -0.932089004 & -0.833521487 \\ \mathrm{H} & 1.845361667 & -0.607638685 & -1.640856042 \\ \mathrm{H} & 1.525914678 & -1.929115680 & -0.534471914 \\ \mathrm{C} & -0.243901214 & -1.094612863 & -1.407931402 \\ \mathrm{H} & -0.928793728 & -1.423923206 & -0.618074133 \\ & -0.207169425 & -1.915109943 & -2.136134096\end{array}$

J

$=$
O $-0.368891356-1.935525921$
$-0.189041833$
O 2.638699734
0.165920084
$-0.371702217$
O -2.132227283
$-1.099310703$
0.998329122
O 1.286572845
$-0.330338002$
1.409200441
N -0.775259531
0.291026874
$-0.275109838$
N 0.484032178
0.620648930
$-0.568168150$
C -2.354795798
3.598224782
$-0.821878173$
C -4.024021610
1.888695639
$-1.160430528$
C -0.419216973
$-3.303896208$
0.382156335
C 3.897863748
$-0.157233558$
C $\quad 0.756502272$
$-3.984177690$
$-0.316053298$
C $-1.749882853-3.953333416$
$-0.002537911$
C -0.202492770
$-3.242573234$
1.895160938
C $4.080419433 \quad 0.748839330$
1.535675739
C $3.926348562-1.643351680$
0.683141667
C $4.943874362 \quad 0.157373064$
C -1.165964749
$-0.985756211$
$-0.757271892$
C 1.462260993
0.073783820
0.279582769
C -1.773367478
1.270619700
0.266766334
C -1.407641813
2.622805173
$-0.548303766$
C $\quad-3.086470926$
$-0.526099799$
C -3.666584457
0.901038228
$-0.872829873$
H -2.585416537
3.238745807
$-1.137838080$
$\mathrm{H} \quad-1.884974163 \quad-3.929420824$
0.472492038
H -1.750389145
$-4.998804435$
$-1.088335723$
H $0.681749604 \quad-2.642486231$
0.321131008
$\mathrm{H} \quad-1.060645147 \quad-2.800127429$
2.120625034
H 0.63319980
$-3.946238378$
2.401693195
H 0.81713788
$-5.031555452$
$-1.401964638$
H $\quad-0.055259175 \quad-4.258747588$
$-0.006648397$
H $1.692882419 \quad-3.486300964$
2.274232500
H $3.987558345 \quad 1.798498658$
$-0.053798183$
H 5.080234707
0.597689102
1.240460192
H 5.947624240
H 4.772421567
$-0.052962300$
1.955160462
H 4.891225617
$-0.451595735$
$-0.376022798$
H 4.908627367
1.211124143
$-1.649435312$
H 3.753155180
$-1.903089628$
$-1.044998318$
H 3.337824884
$-2.253663270$
1.090358895
H $3.164326249-1.872991046$
$-0.208563721$
H $\quad-0.387352141$
2.887992794
2.303071999
H -3.375315811
$-0.142029616$
H -4.404367038
$4.003091917-1.362415809$
H $\quad-2.065881573$
4.644785752
$-0.796731923$
H $\quad-5.040519191$
1.598209539
$-1.408377547$

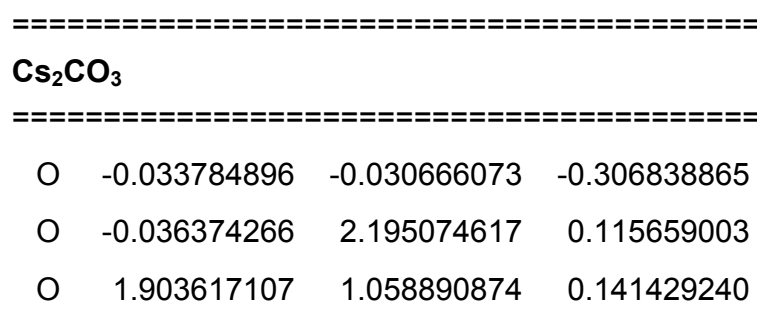




\section{C $\quad 0.623187393 \quad 1.094033072 \quad-0.006555705$ \\ Cs $\quad-2.613469869 \quad 1.261763499 \quad-0.507553813$ \\ Cs $\quad 2.369195581 \quad-1.635902081 \quad-0.479181715$}

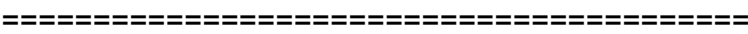

\section{$\mathrm{CsHCO}_{3}$}

\begin{tabular}{|c|c|c|c|}
\hline $\mathrm{O}$ & -0.473984098 & 0.421830118 & 政 \\
\hline V & 0.777242200 & 1.907792869 & -1.0201190 \\
\hline 0 & 1.423259524 & -0.242342627 & -0.83 \\
\hline C & 0.508687282 & 0.765567996 & -0.54930 \\
\hline & 2.09 & 13 & -1 \\
\hline & 1.6604688 & 10315930 & 0.138 \\
\hline
\end{tabular}

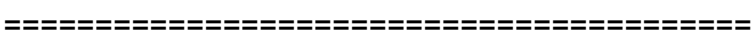

\section{Cs-OTf}

$\begin{array}{cccc}================================== \\ \mathrm{O} & -1.373185812 & 0.184333238 & -2.579804772 \\ \text { S } & -0.751386597 & -0.142371876 & -1.269206511 \\ \text { C } & -1.966965355 & 0.441341017 & 0.002115058 \\ O & -0.610813731 & -1.603574491 & -1.029370528 \\ O & 0.493132198 & 0.624622833 & -0.998120354 \\ \text { F } & -1.507515107 & 0.190198097 & 1.236621532 \\ \text { F } & -3.142023315 & -0.185892821 & -0.152863563 \\ \text { F } & -2.168716037 & 1.761460276 & -0.117769384 \\ \text { Cs } & 1.448562961 & -1.225645069 & -3.580829055\end{array}$

\section{$\mathrm{K}_{3} \mathrm{PO}_{4}$}

$\begin{array}{crrr}=================================== \\ \mathrm{O} & 0.695411203 & 2.214385375 & -1.059714189 \\ \mathrm{P} & 0.586532457 & 2.159566499 & 0.504292069 \\ \mathrm{O} & -0.678310382 & 1.366845955 & 0.995502410 \\ \mathrm{O} & 1.863135132 & 1.317865406 & 1.014439980 \\ \mathrm{O} & 0.701592848 & 3.577238722 & 1.164754480 \\ \mathrm{~K} & 0.376013494 & -0.427890664 & -0.658187275 \\ \mathrm{~K} & 2.862835440 & 3.608633929 & -0.403798581 \\ \mathrm{~K} & 0.380193685 & 2.019328595 & 3.335312552\end{array}$

\section{$\mathrm{K}_{2} \mathrm{HPO}_{4}$}

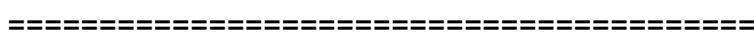

$\begin{array}{llll}\mathrm{O} & 0.336318023 & 2.550694338 & -0.071746172\end{array}$

$\begin{array}{llll}\mathrm{P} & 0.481726281 & 1.771165582 & 1.456284766\end{array}$
O $\quad-0.734627501$
0.854268796
1.595279833
$\begin{array}{llll}\text { O } & 1.880515679 & 1.156796809 & 1.423709444\end{array}$
$\begin{array}{llll}\mathrm{O} & 0.398866381 & 3.031377611 & 2.382021911\end{array}$
H $\quad 0.300165146 \quad 1.841054482 \quad-0.727126482$
K $\quad 2.760656598 \quad 3.593789339 \quad 1.322461973$
$\begin{array}{llll}\text { K } & -1.974279282 & 2.406300289 & 3.149294133\end{array}$

\begin{tabular}{|c|c|c|c|}
\hline \multicolumn{4}{|c|}{ 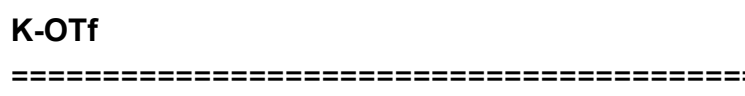 } \\
\hline O & 657417409 & 0.0000 & -3.13 \\
\hline 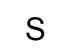 & .693597818 & 0.1000 & -2.15 \\
\hline C & 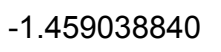 & s.t. & -0.4 \\
\hline $\mathrm{O}$ & -0.468964392 & -1.584174180 & -2.095 \\
\hline 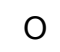 & 0.599815886 & 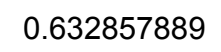 & -2 \\
\hline $\mathrm{F}$ & er & -0.237 & 0178 \\
\hline $\mathrm{F}$ & 2 & -5 & -0.3 \\
\hline $\mathrm{F}$ & 1 & & \\
\hline K & 1. & -1 & -0 \\
\hline \multicolumn{4}{|c|}{ OTf- } \\
\hline $\mathrm{O}$ & -1.039 & 0.03 & $-2 . \varepsilon$ \\
\hline S & -0.373 & -0.31 & -1.6 \\
\hline C & -1.615 & 1 & $-0.3^{2}$ \\
\hline $\mathrm{O}$ & -0.285 & -1.7 & -1.3 \\
\hline 0 & 0.827 & 0.48 & -1.2 \\
\hline$F$ & -1.193024032 & 0.04 & 0.914 \\
\hline$F$ & -2.808922606 & -0.345793773 & -0.49217555 \\
\hline $\mathrm{F}$ & 1.845698706 & 1.605765650 & -0.44965246 \\
\hline
\end{tabular}

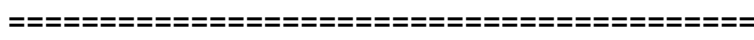

OTf ·

$\begin{array}{cccc}==================================== \\ \mathrm{S} & -1.512587765 & 2.269443852 & 3.443924179 \\ \mathrm{C} & -2.128657691 & 0.792910293 & 4.404686927 \\ \mathrm{O} & -1.380733474 & 3.375102106 & 4.430062016 \\ \mathrm{O} & -2.526782482 & 2.540282219 & 2.401508705 \\ \mathrm{O} & -0.190679817 & 1.880110543 & 2.887859161 \\ \mathrm{~F} & -3.310747235 & 1.093456747 & 4.934826599 \\ \mathrm{~F} & -1.255389941 & 0.508636730 & 5.366402040 \\ \mathrm{~F} & -2.248722013 & -0.240798050 & 3.576454222\end{array}$


XIII. Vibrational Frequencies

Table S5. Vibrational frequencies of optimized structures in $\mathrm{cm}^{-1}$.

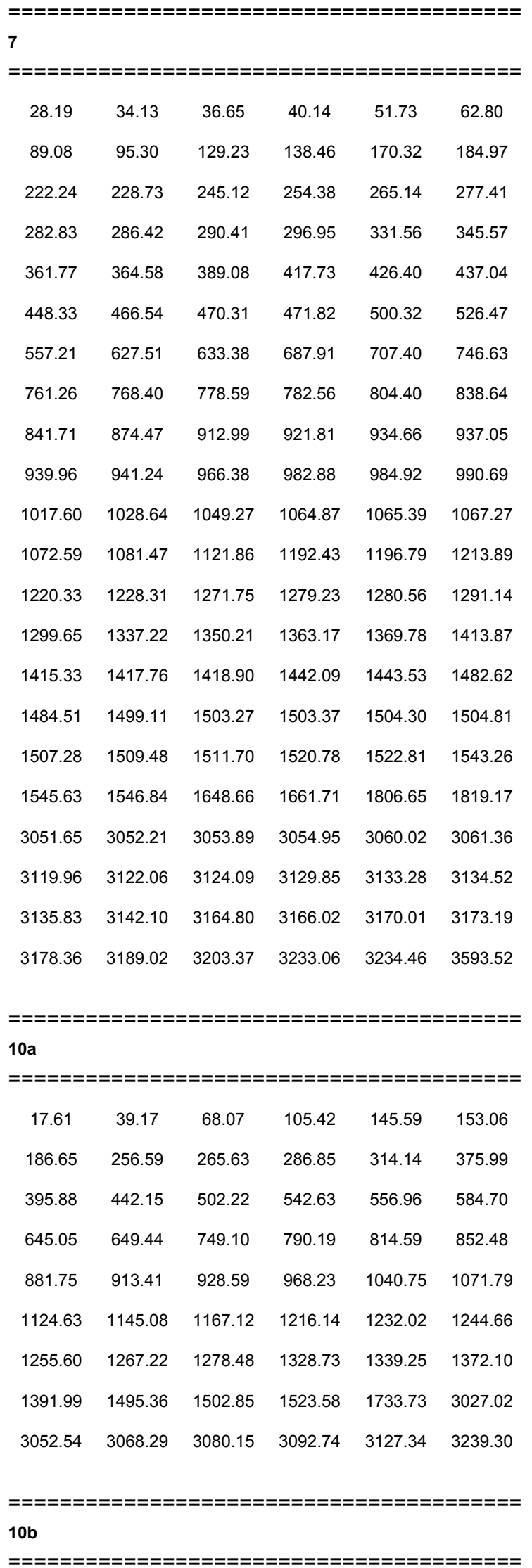

$\begin{array}{rrrrrr}26.26 & 45.56 & 65.46 & 95.53 & 156.58 & 172.98 \\ 193.78 & 255.83 & 261.86 & 280.32 & 307.91 & 317.63 \\ 361.80 & 393.90 & 449.68 & 471.41 & 482.06 & 531.03 \\ 544.04 & 565.80 & 584.96 & 668.33 & 734.90 & 771.71 \\ 802.87 & 829.53 & 871.87 & 889.90 & 933.89 & 950.18 \\ 990.03 & 1058.93 & 1087.37 & 1097.50 & 1109.33 & 1125.97 \\ 1173.74 & 1203.46 & 1218.77 & 1253.48 & 1261.25 & 1274.30 \\ 1296.47 & 1327.46 & 1376.47 & 1379.91 & 1390.79 & 1392.43 \\ 1402.25 & 1485.39 & 1494.56 & 1505.12 & 1515.30 & 1756.87 \\ 3016.73 & 3038.23 & 3043.06 & 3046.15 & 3063.17 & 3083.86 \\ 3093.43 & 3099.14 & 3199.41 & & & \end{array}$

\begin{tabular}{|c|c|c|c|c|c|}
\hline \multicolumn{6}{|l|}{$10 c$} \\
\hline =ニニニニニ & $====$ & $:====$ & $=====$ & =ニニニニ & =ニニニニ= \\
\hline 22.05 & 39.38 & 48.15 & 57.87 & 109.82 & 137.36 \\
\hline 182.79 & 221.22 & 251.61 & 255.35 & 272.60 & 304.33 \\
\hline 306.75 & 338.01 & 354.20 & 386.81 & 449.96 & 481.88 \\
\hline 500.81 & 539.34 & 550.45 & 573.35 & 585.90 & 667.50 \\
\hline 721.93 & 748.86 & 782.29 & 805.90 & 842.23 & 879.51 \\
\hline 888.62 & 919.95 & 966.23 & 1005.28 & 1019.49 & 1055.14 \\
\hline 1080.03 & 1104.10 & 1122.13 & 1140.23 & 1182.25 & 1222.14 \\
\hline 1232.04 & 1255.46 & 1264.52 & 1272.08 & 1290.95 & 1309.08 \\
\hline 1343.98 & 1363.25 & 1377.13 & 1387.38 & 1401.61 & 1406.83 \\
\hline 1410.08 & 1489.13 & 1492.49 & 1497.97 & 1512.78 & 1518.72 \\
\hline 1748.28 & 3015.86 & 3029.80 & 3034.23 & 3041.71 & 3046.96 \\
\hline 3065.38 & 3072.65 & 3079.26 & 3086.06 & 3092.48 & 3191.37 \\
\hline
\end{tabular}

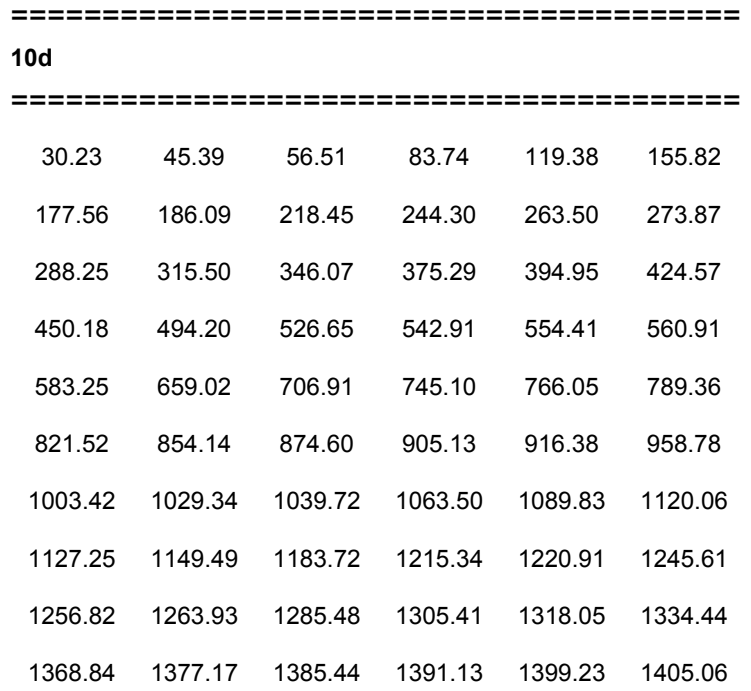




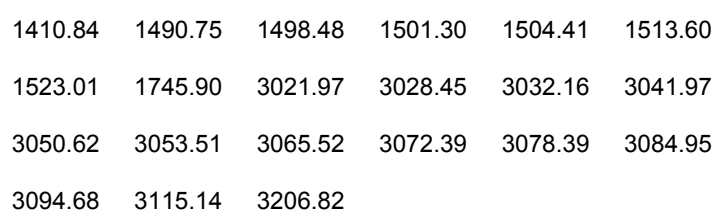

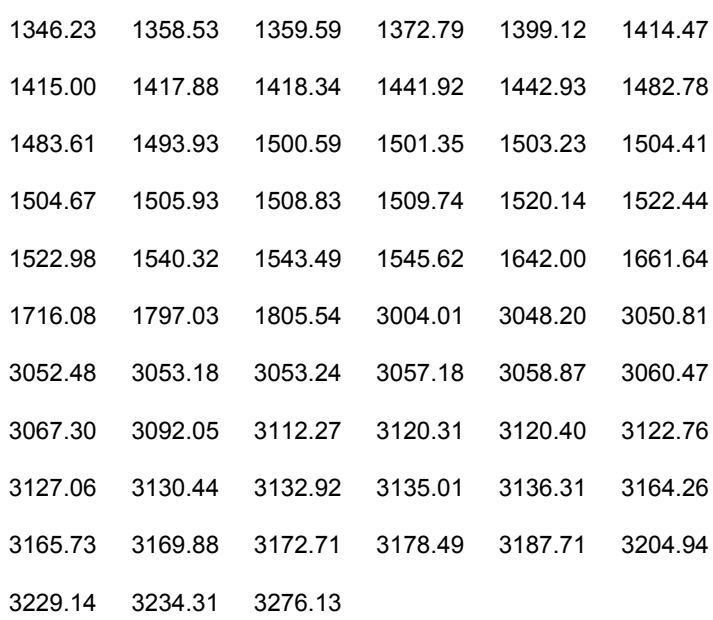

\begin{tabular}{|c|c|c|c|c|c|}
\hline 25.07 & 30.20 & 36.11 & 39.97 & 42.45 & 46.45 \\
\hline 51.78 & 52.68 & 71.39 & 90.46 & 97.51 & 133.17 \\
\hline 146.88 & 150.05 & 181.75 & 196.99 & 219.81 & 227.36 \\
\hline 228.78 & 233.90 & 255.94 & 256.95 & 263.66 & 272.61 \\
\hline 278.25 & 291.08 & 294.21 & 307.60 & 337.68 & 339.65 \\
\hline 350.21 & 362.61 & 365.02 & 372.37 & 416.53 & 425.77 \\
\hline 443.48 & 444.67 & 457.31 & 465.79 & 471.06 & 475.27 \\
\hline 485.73 & 519.64 & 530.52 & 548.07 & 556.23 & 631.31 \\
\hline 642.80 & 694.24 & 709.24 & 741.88 & 746.39 & 761.78 \\
\hline 766.91 & 778.01 & 790.78 & 812.86 & 824.51 & 837.01 \\
\hline 840.44 & 862.99 & 875.00 & 886.19 & 911.78 & 920.39 \\
\hline 934.63 & 937.30 & 937.92 & 939.50 & 940.11 & 950.01 \\
\hline 965.44 & 982.92 & 984.26 & 986.29 & 989.89 & 1018.06 \\
\hline 1026.80 & 1053.64 & 1064.49 & 1065.16 & 1065.53 & 1067.44 \\
\hline 1071.47 & 1084.34 & 1095.78 & 1104.16 & 1121.93 & 1169.46 \\
\hline 1196.22 & 1201.35 & 1207.42 & 1208.86 & 1220.38 & 1221.59 \\
\hline 1261.87 & 1273.53 & 1278.69 & 1280.95 & 1286.00 & 1291.50 \\
\hline 1299.12 & 1312.24 & 1331.14 & 1344.18 & 1359.86 & 1365.99 \\
\hline 1374.61 & 1379.57 & 1384.05 & 1393.10 & 1403.56 & 1413.07 \\
\hline 1414.92 & 1417.27 & 1418.61 & 1441.05 & 1442.90 & 1480.03 \\
\hline 1483.12 & 1484.00 & 1488.10 & 1498.94 & 1503.12 & 1503.71 \\
\hline 1504.60 & 1504.89 & 1505.40 & 1509.00 & 1509.90 & 1514.08 \\
\hline 1520.81 & 1523.13 & 1543.24 & 1544.11 & 1546.44 & 1647.66 \\
\hline 1660.68 & 1746.51 & 1790.89 & 1805.51 & 3006.48 & 3032.35 \\
\hline 3035.76 & 3039.38 & 3050.45 & 3050.85 & 3052.20 & 3053.93 \\
\hline 3055.07 & 3058.67 & 3060.70 & 3083.00 & 3086.14 & 3092.40 \\
\hline 3118.49 & 3119.91 & 3122.24 & 3130.87 & 3132.20 & 3133.51 \\
\hline 3135.45 & 3139.35 & 3164.57 & 3166.41 & 3171.00 & 3171.03 \\
\hline
\end{tabular}


$\begin{array}{llllll}3178.27 & 3183.02 & 3188.83 & 3203.43 & 3230.58 & 3235.87\end{array}$

\begin{tabular}{|c|c|c|c|c|c|}
\hline 21.61 & 25.63 & 31.39 & 36.85 & 38.26 & 41.72 \\
\hline 47.57 & 50.13 & 67.02 & 85.87 & 97.19 & 115.57 \\
\hline 133.16 & 150.06 & 175.48 & 181.90 & 198.77 & 219.67 \\
\hline 228.42 & 229.63 & 233.81 & 252.53 & 257.63 & 267.82 \\
\hline 272.86 & 277.72 & 290.85 & 293.13 & 300.51 & 321.12 \\
\hline 338.28 & 339.15 & 351.42 & 361.52 & 365.74 & 381.75 \\
\hline 416.39 & 420.87 & 442.49 & 453.61 & 457.70 & 465.58 \\
\hline 472.80 & 478.38 & 487.26 & 510.98 & 527.56 & 534.52 \\
\hline 585.97 & 631.31 & 645.16 & 699. & 706.25 & 717.12 \\
\hline 744.78 & 758. & 762.84 & 772.21 & 780.46 & 785.96 \\
\hline 810.95 & 838.34 & 840.63 & 850.18 & 875.10 & 879.95 \\
\hline 884.10 & 897.75 & 915.60 & 931.46 & 934.49 & 938.18 \\
\hline 939.89 & 940.43 & 964.13 & 965.99 & 982.68 & 986.44 \\
\hline 989.98 & 1001.09 & 1006.54 & 1018.81 & 1041.23 & 1055.95 \\
\hline 1064.20 & 064.98 & 1067.31 & 1068.74 & 1078.13 & 083.62 \\
\hline 1100.78 & 120 & 1139 & 117 & 119 & 1201.61 \\
\hline 1208.2 & 217 & 1221 & 1232.09 & 27 & 71.36 \\
\hline 1278. & 1281.25 & 1287.64 & 1291.34 & 1296.56 & 1307.93 \\
\hline 1309.86 & 1341.45 & 1351.43 & 1362.83 & 1366.75 & 1375.47 \\
\hline 1382.11 & 1387.07 & 1401.49 & 1408.20 & 1412.55 & 1414.90 \\
\hline 1416.17 & 1416.78 & 1418.80 & 1440.63 & 1442.94 & 1482.27 \\
\hline 483.46 & 1484 & 1490 & 149 & 149 & 4 \\
\hline 504. & 15 & 1 & 15 & 8 & 151 \\
\hline 518. & 1520 & 152 & 4 & 1 & 154 \\
\hline 1648.77 & 1661.15 & 1737.94 & 1783.53 & 1806.49 & 3012.06 \\
\hline 3017.11 & 3024.28 & 3035.53 & 3042.95 & 3050.09 & 3050.33 \\
\hline 3051.43 & 3053.88 & 3057.44 & 3057.89 & 3060.69 & 3060.75 \\
\hline 078. & 3086 & 3103 & 118 & 311 & 312 \\
\hline 31. & 3131 & 313 & 313 & 313 & 316 \\
\hline 66 & 316 & 3169.73 & 3171.53 & 3178.25 & 3188.90 \\
\hline 203. & 3228.03 & & & & \\
\hline
\end{tabular}

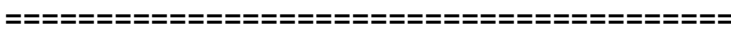

11d

\begin{tabular}{|c|c|c|c|c|c|}
\hline 14.89 & 21.39 & 25.90 & 33.22 & 40.57 & 42.74 \\
\hline 52.00 & 68.57 & 79.43 & 92.98 & 106.48 & 116.32 \\
\hline 139.81 & 148.12 & 171.51 & 195.82 & 208.50 & 220.32 \\
\hline 230.88 & 236.18 & 241.10 & 244.95 & 251.14 & 260.07 \\
\hline
\end{tabular}

\begin{tabular}{|c|c|c|c|c|c|}
\hline 265.09 & 273.19 & 286.35 & 295.34 & 310.04 & 315.38 \\
\hline 332.49 & 343.14 & 356.20 & 364.57 & 370.36 & 386.08 \\
\hline 395.27 & 415.22 & 420.48 & 441.96 & 445.49 & 457.08 \\
\hline 462.46 & 468.52 & 470.10 & 478.53 & 492.42 & 511.04 \\
\hline 540.10 & 558.76 & 598.97 & 631.02 & 641.83 & 668.50 \\
\hline 704.45 & 722.62 & 743.82 & 747.35 & 763.38 & 768.14 \\
\hline 772.19 & 792.76 & 796.79 & 826.28 & 834.45 & 849.48 \\
\hline 852.82 & 871.72 & 874.84 & 890.96 & 905.71 & 916.04 \\
\hline 920.98 & 935.37 & 937.58 & 938.89 & 939.45 & 964.97 \\
\hline 976.13 & 982.56 & 984.93 & 997.31 & 1010.10 & 1011.83 \\
\hline 1016.17 & 1036.58 & 1045.77 & 1057.19 & 1064.93 & 1065.37 \\
\hline 1068.33 & 1070.09 & 1081.32 & 1103.04 & 1115.65 & 1127.30 \\
\hline 1140.91 & 1181.44 & 1197.19 & 1200.34 & 1204.92 & 1216.54 \\
\hline 1217.98 & 1226.37 & 1238.11 & 1269.67 & 1278.18 & 1280.29 \\
\hline 1282.43 & 1289.44 & 1291.78 & 1296.24 & 1312.60 & 1318.33 \\
\hline 1339.71 & 1343.64 & 1350.72 & 1356.80 & 1369.94 & 1375.03 \\
\hline 1377.33 & 1390.29 & 1401.68 & 1403.80 & 1414.15 & 1414.88 \\
\hline 1417.63 & 1418.09 & 1422.49 & 1442.30 & 1443.13 & 1482.74 \\
\hline 1483.36 & 1496.18 & 1497.92 & 1499.87 & 1501.40 & 1503.65 \\
\hline 1503.90 & 1504.15 & 1507.17 & 1508.65 & 1509.01 & 1510.20 \\
\hline 1518.01 & 1521.65 & 1523.54 & 1526.29 & 1537.59 & 1544.22 \\
\hline 1546.05 & 1639.80 & 1660.84 & 1724.11 & 1789.04 & 1801.56 \\
\hline 3018.06 & 3022.27 & 3029.89 & 3036.01 & 3042.98 & 3045.49 \\
\hline 3048.85 & 3051.65 & 3053.05 & 3053.13 & 3058.36 & 3059.73 \\
\hline 3059.99 & 3064.23 & 3078.62 & 3084.85 & 3118.58 & 3119.41 \\
\hline 3120.25 & 3122.26 & 3125.66 & 3127.07 & 3130.58 & 3134.32 \\
\hline 3135.80 & 3139.89 & 3164.30 & 3170.81 & 3170.87 & 3175.26 \\
\hline 3177.74 & 3186.87 & 3192.13 & 3204.49 & 3230.65 & 3286.58 \\
\hline \multicolumn{6}{|c|}{ 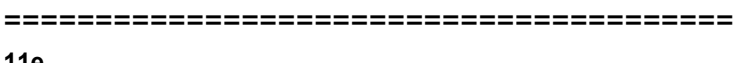 } \\
\hline \multicolumn{6}{|c|}{ 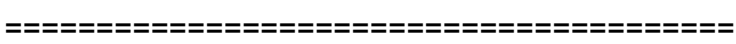 } \\
\hline 20.44 & 29.10 & 31.89 & 38.11 & 39.11 & 42.07 \\
\hline 47.89 & 55.06 & 81.15 & 86.29 & 101.36 & 107.85 \\
\hline 128.94 & 145.85 & 150.07 & 165.76 & 174.65 & 198.60 \\
\hline 212.73 & 226.30 & 226.79 & 229.66 & 235.38 & 244.23 \\
\hline 256.85 & 268.12 & 272.10 & 285.96 & 290.16 & 301.31 \\
\hline 308.89 & 329.87 & 346.34 & 353.66 & 362.70 & 365.21 \\
\hline 369.01 & 383.44 & 404.97 & 409.02 & 418.37 & 439.91 \\
\hline 444.05 & 457.09 & 464.00 & 468.74 & 474.15 & 484.14 \\
\hline 496.44 & 500.19 & 537.61 & 566.47 & 611.83 & 632.39 \\
\hline 642.88 & 661.08 & 700.13 & 713.26 & 735.87 & 749.58 \\
\hline 763.34 & 768.56 & 775.01 & 785.71 & 790.70 & 799.64 \\
\hline
\end{tabular}




\begin{tabular}{|c|c|c|c|c|c|}
\hline 819.75 & 839.49 & 840.27 & 848.42 & 875.98 & 878.26 \\
\hline 892.70 & 911.02 & 933.94 & 934.53 & 937.54 & 938.11 \\
\hline 939.56 & 949.04 & 960.67 & 969.46 & 982.73 & 984.39 \\
\hline 985.29 & 991.72 & 1006.57 & 1014.35 & 1037.31 & 1043.61 \\
\hline 1055.72 & 1064.45 & 1065.92 & 1067.72 & 1069.13 & 1080.90 \\
\hline 1086.26 & 1095.53 & 1124.27 & 1127.92 & 1132.52 & 1182.97 \\
\hline 1198.62 & 1202.64 & 1212.05 & 1216.87 & 1221.61 & 1228.50 \\
\hline 1233.64 & 1252.51 & 1272.53 & 1278.21 & 1280.25 & 1283.28 \\
\hline 1289.23 & 1300.02 & 1304.83 & 1316.57 & 1336.36 & 1341.07 \\
\hline 1346.25 & 1350.59 & 1359.38 & 1363.21 & 1369.98 & 1373.77 \\
\hline 1389.14 & 1391.34 & 1406.87 & 1413.64 & 1415.38 & 1417.04 \\
\hline 1417.41 & 1417.80 & 1423.40 & 1441.70 & 1442.59 & 1482.63 \\
\hline 1483.66 & 1491.60 & 1498.26 & 1499.96 & 1501.23 & 1503.30 \\
\hline 1503.71 & 1504.40 & 1504.72 & 1505.79 & 1508.87 & 1509.90 \\
\hline 1513.76 & 1515.14 & 1521.15 & 1522.59 & 1524.28 & 1541.99 \\
\hline 1543.62 & 1545.51 & 1643.38 & 1661.29 & 1736.16 & 1790.73 \\
\hline 1804.46 & 3009.34 & 3025.69 & 3031.47 & 3033.35 & 3034.84 \\
\hline 3040.44 & 3046.95 & 3048.39 & 3050.21 & 3051.37 & 3052.44 \\
\hline 3056.89 & 3057.97 & 3059.05 & 3067.18 & 3072.70 & 3076.23 \\
\hline 3098.98 & 3102.09 & 3118.11 & 3119.38 & 3121.04 & 3122.53 \\
\hline 3126.07 & 3129.36 & 3132.91 & 3135.21 & 3135.60 & 3167.20 \\
\hline 3168.09 & 3171.12 & 3171.47 & 3178.88 & 3188.61 & 3196.37 \\
\hline 3205.02 & 3238.66 & 3262 & & & \\
\hline
\end{tabular}

\begin{tabular}{|c|c|c|c|c|c|}
\hline \multicolumn{6}{|l|}{ A } \\
\hline$===ニ==$ & $====$ & $====$ & $:====$ & $====$ & $==ニ=ニ$ \\
\hline 10.42 & 13.87 & 20.00 & 27.09 & 31.61 & 40.69 \\
\hline 45.65 & 48.56 & 55.87 & 58.47 & 77.23 & 80.42 \\
\hline 99.28 & 106.10 & 109.21 & 124.76 & 130.61 & 132.55 \\
\hline 143.24 & 143.58 & 163.07 & 179.53 & 196.63 & 202.15 \\
\hline 203.69 & 211.08 & 223.81 & 227.55 & 230.96 & 236.37 \\
\hline 238.12 & 248.46 & 254.34 & 265.62 & 269.39 & 270.67 \\
\hline 275.07 & 278.73 & 280.16 & 281.60 & 288.57 & 293.09 \\
\hline 297.46 & 298.96 & 303.59 & 304.24 & 305.61 & 328.89 \\
\hline 329.67 & 339.34 & 353.84 & 360.48 & 366.53 & 368.43 \\
\hline 372.19 & 372.67 & 388.23 & 397.63 & 399.60 & 401.78 \\
\hline 423.66 & 433.53 & 434.90 & 436.35 & 438.85 & 446.67 \\
\hline 449.77 & 466.70 & 476.10 & 482.00 & 484.24 & 486.42 \\
\hline 501.26 & 525.45 & 534.91 & 573.92 & 583.44 & 584.70 \\
\hline 631.47 & 637.18 & 693.24 & 705.00 & 741.09 & 760.37 \\
\hline 762.35 & 770.96 & 775.23 & 800.75 & 813.40 & 813.92 \\
\hline 815.21 & 838.77 & 844.29 & 885.74 & 898.57 & 924.93 \\
\hline
\end{tabular}

\begin{tabular}{|c|c|c|c|c|c|}
\hline 928.79 & 934.83 & 937.33 & 937.37 & 939.54 & 940.34 \\
\hline 950.76 & 952.13 & 952.61 & 958.74 & 959.42 & 971.46 \\
\hline 974.13 & 975.92 & 976.53 & 981.33 & 985.01 & 1009.03 \\
\hline 1023.60 & 1040.58 & 1046.32 & 1046.65 & 1054.43 & 1055.04 \\
\hline 1055.98 & 1059.85 & 1060.88 & 1065.41 & 1067.39 & 1072.42 \\
\hline 1085.69 & 1114.62 & 1185.12 & 1190.47 & 1205.79 & 1206.38 \\
\hline 1208.28 & 1213.44 & 1215.00 & 1218.31 & 1218.57 & 1221.29 \\
\hline 1232.96 & 1233.59 & 1234.72 & 1236.83 & 1266.25 & 1275.72 \\
\hline 1280.22 & 1283.74 & 1293.97 & 1344.67 & 1346.20 & 1363.54 \\
\hline 1380.52 & 1398.35 & 1404.07 & 1405.05 & 1405.54 & 1406.72 \\
\hline 1407.17 & 1412.16 & 1412.49 & 1414.22 & 1415.80 & 1429.85 \\
\hline 1437.70 & 1438.47 & 1438.99 & 1448.93 & 1479.35 & 1482.22 \\
\hline 1486.68 & 1487.41 & 1488.06 & 1494.55 & 1498.48 & 1500.27 \\
\hline 1501.15 & 1502.10 & 1502.66 & 1502.89 & 1505.01 & 1506.57 \\
\hline 1507.66 & 1509.15 & 1511.70 & 1511.93 & 1518.14 & 1518.63 \\
\hline 1519.49 & 1523.40 & 1525.37 & 1529.66 & 1530.03 & 1533.42 \\
\hline 1536.75 & 1543.73 & 1544.54 & 1546.16 & 1547.31 & 1550.45 \\
\hline 1628.77 & 1654.11 & 1719.19 & 1748.56 & 3000.15 & 3003.75 \\
\hline 3006.36 & 3007.85 & 3008.06 & 3009.97 & 3015.49 & 3016.76 \\
\hline 3020.51 & 3030.08 & 3032.43 & 3034.02 & 3039.15 & 3040.84 \\
\hline 3046.56 & 3086.16 & 3088.34 & 3088.98 & 3094.76 & 3095.33 \\
\hline 3096.26 & 3097.17 & 3098.29 & 3100.45 & 3101.74 & 3102.26 \\
\hline 3103.56 & 3113.93 & 3116.83 & 3124.54 & 3126.78 & 3130.69 \\
\hline 3132.37 & 3133.98 & 3137.53 & 3137.95 & 3142.13 & 3143.03 \\
\hline 3151.15 & 3159.13 & 3159.29 & 3160.03 & 3162.47 & 3162.60 \\
\hline 3162.82 & 3164.41 & 3165.28 & 3181.50 & 3246.45 & 3266.59 \\
\hline \multicolumn{6}{|c|}{ 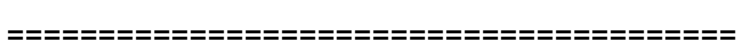 } \\
\hline \multirow{2}{*}{\multicolumn{6}{|c|}{ 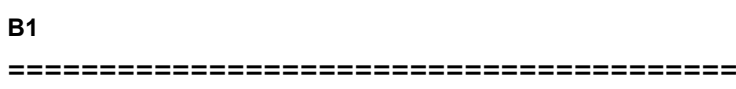 }} \\
\hline & & & & & \\
\hline 12.67 & 17.34 & 25.56 & 26.88 & 31.12 & 37.49 \\
\hline 45.39 & 48.48 & 52.25 & 59.62 & 62.00 & 69.63 \\
\hline 73.25 & 75.79 & 80.76 & 84.03 & 84.64 & 90.91 \\
\hline 94.10 & 100.22 & 104.85 & 107.31 & 111.69 & 122.04 \\
\hline 130.33 & 132.28 & 138.68 & 149.95 & 156.98 & 166.46 \\
\hline 173.06 & 176.69 & 188.40 & 190.30 & 192.66 & 204.80 \\
\hline 207.57 & 216.55 & 222.14 & 224.78 & 228.94 & 234.26 \\
\hline 235.36 & 240.32 & 251.92 & 256.91 & 261.04 & 263.52 \\
\hline 264.50 & 268.12 & 273.27 & 276.41 & 278.17 & 280.78 \\
\hline 282.78 & 291.66 & 295.13 & 296.49 & 299.40 & 303.08 \\
\hline 306.32 & 306.82 & 311.36 & 319.58 & 326.76 & 339.95 \\
\hline 347.45 & 356.27 & 358.63 & 366.12 & 370.47 & 372.89 \\
\hline 373.92 & 382.56 & 398.39 & 399.40 & 402.40 & 418.77 \\
\hline
\end{tabular}




\begin{tabular}{|c|c|c|c|c|c|}
\hline 425.09 & 429.48 & 433.93 & 436.42 & 441.59 & 444.53 \\
\hline 460.27 & 464.20 & 470.88 & 476.40 & 480.14 & 482.24 \\
\hline 485.85 & 487.92 & 508.75 & 511.23 & 525.95 & 528.94 \\
\hline 545.55 & 551.26 & 572.09 & 573.09 & 590.62 & 594.64 \\
\hline 630.09 & 631.80 & 637.04 & 660.21 & 684.55 & 700.93 \\
\hline 742.13 & 745.39 & 757.36 & 759.82 & 776.49 & 777.92 \\
\hline 795.89 & 798.95 & 801.71 & 816.24 & 819.77 & 820.82 \\
\hline 830.06 & 844.15 & 865.48 & 883.30 & 888.18 & 901.08 \\
\hline 924.37 & 926.58 & 930.06 & 933.61 & 934.48 & 937.66 \\
\hline 940.04 & 941.47 & 943.17 & 946.18 & 949.22 & 949.38 \\
\hline 950.80 & 952.17 & 959.87 & 973.69 & 976.12 & 976.27 \\
\hline 979.41 & 980.93 & 984.16 & 1014.68 & 1017.00 & 1018.37 \\
\hline 1040.88 & 1045.14 & 1047.34 & 1047.49 & 1051.94 & 1054.40 \\
\hline 1057.13 & 1061.40 & 1062.16 & 1064.68 & 1066.88 & 1070.16 \\
\hline 1078.15 & 1092.41 & 1114.39 & 1120.16 & 1159.32 & 1174.27 \\
\hline 1191.11 & 1194.50 & 1204.89 & 1207.67 & 1208.91 & 1211.90 \\
\hline 1215.54 & 1216.56 & 1217.31 & 1218.44 & 1220.06 & 1229.59 \\
\hline 1230.30 & 1233.03 & 1234.06 & 1235.28 & 1243.65 & 1261.46 \\
\hline 1264.37 & 1269.42 & 1275.38 & 1279.58 & 1284.13 & 1296.26 \\
\hline 1308.17 & 1326.84 & 1331.50 & 1333.88 & 1346.15 & 1359.54 \\
\hline 1369.17 & 1380.79 & 1400.51 & 1406.09 & 1407.17 & 1408.05 \\
\hline 1409.37 & 1411.73 & 1414.83 & 1415.88 & 1418.74 & 1423.71 \\
\hline 1426.86 & 1432.78 & 1438.56 & 1443.18 & 1448.50 & 1458.15 \\
\hline 1480.62 & 1482.01 & 1488.42 & 1489.63 & 1493.56 & 1494.43 \\
\hline 1496.68 & 1497.20 & 1498.40 & 1500.51 & 1501.47 & 1503.07 \\
\hline 1504.36 & 1505.21 & 1506.24 & 1506.99 & 1508.03 & 1508.80 \\
\hline 1513.13 & 1514.43 & 1516.63 & 1519.02 & 1521.53 & 1523.44 \\
\hline 1525.04 & 1526.92 & 1529.72 & 1530.30 & 1534.57 & 1542.61 \\
\hline 1544.61 & 1545.70 & 1548.36 & 1548.86 & 1550.00 & 1642.31 \\
\hline 1659.21 & 1712.11 & 1753.94 & 2987.17 & 3005.26 & 3016.63 \\
\hline 3017.04 & 3017.52 & 3017.97 & 3021.72 & 3023.96 & 3024.00 \\
\hline 3032.88 & 3034.47 & 3035.15 & 3036.14 & 3037.77 & 3041.99 \\
\hline 3046.66 & 3050.99 & 3056.79 & 3063.45 & 3089.38 & 3090.74 \\
\hline 3100.77 & 3102.21 & 3102.47 & 3102.97 & 3105.23 & 3107.63 \\
\hline 3108.43 & 3109.79 & 3117.56 & 3117.66 & 3120.90 & 3126.71 \\
\hline 3131.36 & 3134.81 & 3139.23 & 3141.39 & 3145.96 & 3147.12 \\
\hline 3147.16 & 3148.48 & 3151.16 & 3159.14 & 3161.04 & 3162.24 \\
\hline 3162.43 & 3168.26 & 3169.43 & 3172.34 & 3176.88 & 3187.71 \\
\hline 3193.67 & 3203.25 & 3210.41 & 3216.12 & 3229.29 & 3244.67 \\
\hline \multicolumn{6}{|c|}{ 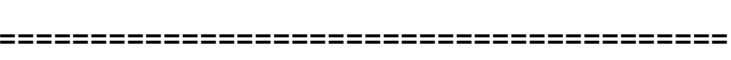 } \\
\hline & & & & & \\
\hline
\end{tabular}

\begin{tabular}{|c|c|c|c|c|c|}
\hline-163.54 & 14.58 & 25.64 & 28.21 & 33.43 & 38.50 \\
\hline 45.33 & 48.60 & 50.25 & 53.29 & 60.09 & 62.71 \\
\hline 67.12 & 71.70 & 75.09 & 77.40 & 79.35 & 85.36 \\
\hline 88.46 & 90.84 & 94.77 & 97.65 & 104.13 & 106.06 \\
\hline 112.00 & 124.55 & 130.08 & 133.82 & 138.38 & 146.79 \\
\hline 152.87 & 159.57 & 164.75 & 190.19 & 196.75 & 201.11 \\
\hline 205.40 & 209.80 & 214.41 & 214.82 & 221.65 & 223.75 \\
\hline 230.34 & 233.56 & 235.34 & 240.58 & 251.76 & 255.82 \\
\hline 260.12 & 262.70 & 265.96 & 274.92 & 276.39 & 278.74 \\
\hline 281.28 & 282.67 & 290.25 & 296.05 & 298.31 & 302.80 \\
\hline 303.88 & 305.66 & 307.19 & 317.97 & 319.04 & 326.46 \\
\hline 335.25 & 338.93 & 343.27 & 344.44 & 351.13 & 362.23 \\
\hline 363.13 & 371.18 & 374.11 & 383.00 & 392.29 & 397.69 \\
\hline 400.20 & 415.45 & 431.15 & 434.54 & 436.04 & 437.04 \\
\hline 446.56 & 464.65 & 468.58 & 471.89 & 474.96 & 478.92 \\
\hline 485.62 & 487.24 & 488.32 & 497.11 & 511.77 & 513.84 \\
\hline 528.70 & 554.52 & 555.17 & 573.58 & 592.99 & 594.90 \\
\hline 600.24 & 616.62 & 631.55 & 639.57 & 685.05 & 700.74 \\
\hline 720.36 & 739.81 & 741.34 & 751.82 & 756.56 & 760.63 \\
\hline 775.70 & 781.66 & 801.22 & 816.37 & 818.53 & 823.06 \\
\hline 827.44 & 836.07 & 847.24 & 882.11 & 885.18 & 909.15 \\
\hline 912.57 & 927.15 & 930.44 & 935.25 & 937.44 & 939.24 \\
\hline 941.12 & 943.59 & 944.63 & 947.17 & 948.42 & 952.16 \\
\hline 952.76 & 961.92 & 963.69 & 968.67 & 974.25 & 975.81 \\
\hline 978.16 & 982.57 & 984.50 & 1002.01 & 1015.25 & 1021.51 \\
\hline 1029.05 & 1042.13 & 1046.35 & 1050.65 & 1054.96 & 1055.85 \\
\hline 1056.96 & 1061.62 & 1063.00 & 1063.80 & 1066.30 & 1067.50 \\
\hline 1070.73 & 1079.17 & 1113.45 & 1134.18 & 1148.44 & 1180.31 \\
\hline 1184.36 & 1192.27 & 1202.90 & 1205.88 & 1208.37 & 1209.51 \\
\hline 1210.19 & 1213.24 & 1215.92 & 1217.61 & 1218.68 & 1219.07 \\
\hline 1220.27 & 1226.04 & 1229.99 & 1231.48 & 1232.87 & 1234.89 \\
\hline 1239.03 & 1267.11 & 1268.70 & 1276.48 & 1279.46 & 1284.96 \\
\hline 1294.75 & 1302.04 & 1307.86 & 1335.22 & 1342.87 & 1347.65 \\
\hline 1366.55 & 1383.28 & 1402.32 & 1407.06 & 1407.95 & 1410.29 \\
\hline 1412.59 & 1415.32 & 1415.86 & 1421.00 & 1422.54 & 1427.58 \\
\hline 1431.91 & 1439.27 & 1448.68 & 1454.37 & 1461.09 & 1479.26 \\
\hline 1481.94 & 1482.49 & 1490.58 & 1491.05 & 1493.81 & 1494.89 \\
\hline 1497.71 & 1498.00 & 1499.10 & 1500.08 & 1502.01 & 1503.41 \\
\hline 1504.33 & 1506.06 & 1506.42 & 1507.75 & 1510.56 & 1511.97 \\
\hline 1513.36 & 1514.20 & 1518.97 & 1519.72 & 1522.31 & 1523.03 \\
\hline 1524.71 & 1526.47 & 1530.38 & 1531.94 & 1534.27 & 1542.26 \\
\hline 1544.5 & 1545.37 & 1546.97 & 1550.59 & 1551.31 & 1649.06 \\
\hline
\end{tabular}


$\begin{array}{llllll}1661.99 & 1713.04 & 1760.65 & 3000.21 & 3011.85 & 3020.75 \\ 3022.13 & 3022.18 & 3024.26 & 3026.65 & 3027.61 & 3033.84 \\ 3036.40 & 3036.56 & 3040.21 & 3041.16 & 3044.89 & 3048.77 \\ 3049.82 & 3053.64 & 3059.28 & 3064.03 & 3093.22 & 3102.51 \\ 3102.76 & 3104.31 & 3105.22 & 3109.87 & 3110.72 & 3114.35 \\ 3115.68 & 3116.16 & 3118.68 & 3120.50 & 3120.82 & 3125.11 \\ 3130.11 & 3131.27 & 3132.78 & 3135.84 & 3138.14 & 3142.02 \\ 3149.40 & 3150.41 & 3152.32 & 3160.43 & 3164.02 & 3164.55 \\ 3165.00 & 3167.80 & 3168.29 & 3169.13 & 3176.71 & 3191.78 \\ 3193.20 & 3211.09 & 3214.89 & 3217.56 & 3226.77 & 3247.59\end{array}$

$=$
B5

\begin{tabular}{|c|c|c|c|c|c|}
\hline 13.73 & 19.28 & 21.28 & 29.84 & 35.70 & 39.43 \\
\hline 43.01 & 48.25 & 51.30 & 55.23 & 57.42 & 63.11 \\
\hline 67.39 & 72.09 & 74.95 & 78.92 & 80.76 & 82.20 \\
\hline 83.68 & 92.18 & 95.60 & 98.79 & 102.88 & 104.83 \\
\hline 115.78 & 118.80 & 123.46 & 135.52 & 142.69 & 145.58 \\
\hline 155.72 & 169.36 & 172.71 & 185.28 & 187.36 & 192.27 \\
\hline 199.23 & 201.22 & 205.85 & 207.70 & 210.33 & 215.73 \\
\hline 220.81 & 226.84 & 230.76 & 232.99 & 236.84 & 239.40 \\
\hline 251.45 & 253.39 & 260.77 & 261.63 & 262.74 & 267.54 \\
\hline 271.88 & 274.23 & 276.45 & 278.11 & 278.65 & 282.75 \\
\hline 293.00 & 296.91 & 298.55 & 301.01 & 303.44 & 305.07 \\
\hline 314.02 & 316.98 & 319.87 & 324.45 & 342.07 & 349.06 \\
\hline 353.08 & 357.38 & 365.20 & 367.70 & 372.00 & 374.82 \\
\hline 378.10 & 384.81 & 391.99 & 397.02 & 400.03 & 406.64 \\
\hline 414.74 & 420.39 & 428.54 & 435.69 & 436.71 & 440.29 \\
\hline 444.45 & 447.23 & 462.42 & 469.14 & 473.57 & 476.53 \\
\hline 479.66 & 483.77 & 488.94 & 494.87 & 501.47 & 507.73 \\
\hline 511.56 & 530.57 & 532.70 & 545.16 & 549.73 & 571.44 \\
\hline 591.49 & 593.10 & 618.81 & 631.60 & 632.86 & 637.88 \\
\hline 674.35 & 684.02 & 702.95 & 722.77 & 742.11 & 756.67 \\
\hline 757.04 & 761.24 & 778.57 & 780.11 & 786.44 & 798.74 \\
\hline 801.29 & 817.55 & 818.86 & 820.00 & 822.32 & 827.85 \\
\hline 835.92 & 840.34 & 844.23 & 864.15 & 884.54 & 907.43 \\
\hline 926.44 & 929.70 & 934.18 & 936.17 & 937.60 & 938.79 \\
\hline 940.89 & 943.00 & 944.69 & 948.96 & 949.50 & 952.01 \\
\hline 952.84 & 955.01 & 959.69 & 964.94 & 974.20 & 975.60 \\
\hline 977.51 & 982.46 & 984.12 & 990.53 & 1015.18 & 1018.86 \\
\hline 1035.53 & 1041.22 & 1041.69 & 1045.94 & 1049.02 & 1052.48 \\
\hline 1054.71 & 1058.25 & 1059.60 & 1061.04 & 1063.52 & 1066.58 \\
\hline
\end{tabular}

\begin{tabular}{|c|c|c|c|c|c|}
\hline 1069.64 & 1078.55 & 1079.00 & 1086.59 & 1111.54 & 1113.47 \\
\hline 1123.36 & 1128.95 & 1172.50 & 1189.91 & 1190.56 & 1205.49 \\
\hline 1207.54 & 1207.68 & 1209.10 & 1210.81 & 1215.74 & 1216.62 \\
\hline 1217.76 & 1218.10 & 1220.55 & 1226.35 & 1229.83 & 1230.31 \\
\hline 1232.95 & 1234.82 & 1237.05 & 1253.42 & 1259.13 & 1267.57 \\
\hline 1275.28 & 1280.17 & 1284.35 & 1289.78 & 1296.42 & 1311.74 \\
\hline 1316.68 & 1326.63 & 1329.12 & 1340.23 & 1342.36 & 1358.68 \\
\hline 1365.19 & 1366.47 & 1379.74 & 1381.89 & 1391.70 & 1397.19 \\
\hline 1400.54 & 1406.30 & 1406.49 & 1406.75 & 1408.09 & 1408.87 \\
\hline 1412.91 & 1415.01 & 1416.80 & 1418.66 & 1422.89 & 1425.81 \\
\hline 1431.35 & 1438.57 & 1444.36 & 1447.47 & 1455.65 & 1476.05 \\
\hline 1481.63 & 1482.15 & 1489.04 & 1490.34 & 1492.46 & 1493.12 \\
\hline 1495.34 & 1496.98 & 1498.73 & 1499.26 & 1500.68 & 1501.27 \\
\hline 1502.63 & 1502.95 & 1503.64 & 1504.33 & 1504.91 & 1505.44 \\
\hline 1506.88 & 1508.31 & 1508.73 & 1513.16 & 1513.97 & 1516.16 \\
\hline 1519.27 & 1520.44 & 1522.37 & 1523.61 & 1524.63 & 1526.65 \\
\hline 1530.29 & 1531.63 & 1533.27 & 1541.67 & 1543.69 & 1546.55 \\
\hline 1547.91 & 1549.83 & 1550.60 & 1640.29 & 1657.25 & 1706.03 \\
\hline 1752.95 & 2991.89 & 3004.31 & 3006.43 & 3008.02 & 3015.66 \\
\hline 3016.36 & 3017.00 & 3019.76 & 3020.98 & 3022.63 & 3023.29 \\
\hline 3028.52 & 3029.97 & 3031.18 & 3034.59 & 3034.86 & 3036.14 \\
\hline 3038.19 & 3042.32 & 3046.90 & 3049.32 & 3050.93 & 3062.94 \\
\hline 3063.61 & 3076.51 & 3089.55 & 3097.02 & 3099.34 & 3102.36 \\
\hline 3102.54 & 3102.89 & 3104.22 & 3105.46 & 3108.08 & 3108.41 \\
\hline 3113.53 & 3116.53 & 3119.31 & 3119.89 & 3122.55 & 3126.48 \\
\hline 3127.64 & 3131.14 & 3132.91 & 3138.90 & 3147.60 & 3149.24 \\
\hline 3151.42 & 3152.65 & 3154.98 & 3158.51 & 3158.73 & 3160.09 \\
\hline 3165.13 & 3167.20 & 3168.40 & 3170.74 & 3171.74 & 3172.30 \\
\hline 3187.26 & 3199.83 & 3200.20 & 3223.43 & 3226.99 & 3240.05 \\
\hline \multicolumn{6}{|c|}{ 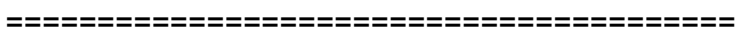 } \\
\hline \multirow{2}{*}{\multicolumn{6}{|c|}{ 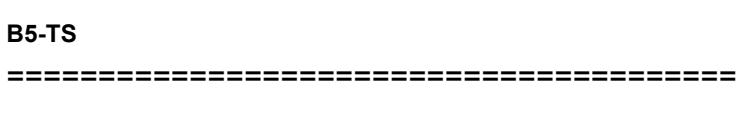 }} \\
\hline & & & & & \\
\hline-84.84 & 12.16 & 19.19 & 25.64 & 28.73 & 34.05 \\
\hline 39.60 & 43.40 & 47.85 & 52.43 & 54.62 & 55.23 \\
\hline 57.83 & 63.46 & 64.47 & 70.14 & 76.85 & 79.01 \\
\hline 82.57 & 88.07 & 94.78 & 101.61 & 104.06 & 109.94 \\
\hline 115.21 & 117.97 & 127.71 & 133.22 & 137.05 & 147.11 \\
\hline 156.25 & 157.78 & 168.70 & 171.06 & 176.15 & 196.23 \\
\hline 198.17 & 198.69 & 203.09 & 205.76 & 209.74 & 211.95 \\
\hline 214.69 & 217.77 & 225.84 & 229.13 & 231.61 & 233.54 \\
\hline 235.21 & 243.84 & 245.36 & 256.06 & 260.30 & 264.67 \\
\hline 268.74 & 271.59 & 273.69 & 275.80 & 276.61 & 279.70 \\
\hline
\end{tabular}




\begin{tabular}{|c|c|c|c|c|c|}
\hline 282.20 & 295.54 & 296.60 & 300.74 & 301.30 & 305.80 \\
\hline 309.11 & 313.57 & 317.79 & 321.72 & 330.37 & 340.45 \\
\hline 343.51 & 350.56 & 352.40 & 363.60 & 365.73 & 370.45 \\
\hline 371.16 & 379.32 & 382.68 & 389.31 & 394.03 & 397.13 \\
\hline 399.97 & 411.95 & 427.34 & 432.91 & 434.66 & 436.83 \\
\hline 439.98 & 442.27 & 469.44 & 471.38 & 473.95 & 477.58 \\
\hline 478.92 & 485.24 & 485.83 & 496.47 & 500.05 & 504.66 \\
\hline 510.25 & 517.90 & 530.06 & 544.53 & 557.88 & 562.15 \\
\hline 572.61 & 594.87 & 596.96 & 604.05 & 631.11 & 639.46 \\
\hline 652.61 & 681.51 & 712.90 & 714.78 & 742.10 & 746.82 \\
\hline 747.52 & 758.00 & 764.97 & 776.97 & 783.86 & 785.72 \\
\hline 803.99 & 806.93 & 818.76 & 820.06 & 821.57 & 823.27 \\
\hline 830.94 & 845.12 & 861.47 & 883.63 & 913.34 & 927.19 \\
\hline 927.75 & 930.04 & 931.83 & 934.48 & 936.77 & 940.51 \\
\hline 942.39 & 943.12 & 948.30 & 950.79 & 952.04 & 952.05 \\
\hline 954.58 & 960.76 & 974.81 & 976.72 & 979.49 & 980.34 \\
\hline 983.25 & 985.17 & 987.22 & 995.97 & 1013.94 & 1020.19 \\
\hline 1031.49 & 1035.86 & 1043.16 & 1047.50 & 1050.10 & 1051.09 \\
\hline 1056.46 & 1058.52 & 1059.14 & 1060.37 & 1061.26 & 1063.23 \\
\hline 1065.85 & 1069.42 & 1076.73 & 1083.51 & 1107.13 & 1111.09 \\
\hline 1117.58 & 1149.03 & 1162.39 & 1177.40 & 1184.77 & 1192.51 \\
\hline 1203.34 & 1204.76 & 1207.22 & 1209.16 & 1209.74 & 1213.12 \\
\hline 1214.16 & 1217.97 & 1218.77 & 1220.37 & 1221.62 & 1229.41 \\
\hline 1231.22 & 1232.00 & 1234.84 & 1237.05 & 1246.82 & 1263.65 \\
\hline 1268.33 & 1275.37 & 1277.04 & 1281.63 & 1288.66 & 1289.22 \\
\hline 1315.21 & 1317.58 & 1322.91 & 1329.08 & 1334.94 & 1359.35 \\
\hline 1360.01 & 1362.50 & 1369.30 & 1386.64 & 1386.85 & 1396.23 \\
\hline 1401.91 & 1406.58 & 1406.82 & 1408.77 & 1409.53 & 1411.26 \\
\hline 1414.28 & 1414.85 & 1417.06 & 1420.18 & 1420.86 & 1426.58 \\
\hline 1431.45 & 1438.07 & 1447.80 & 1453.34 & 1457.95 & 1461.19 \\
\hline 1480.85 & 1481.49 & 1488.54 & 1490.36 & 1490.58 & 1492.23 \\
\hline 1494.10 & 1495.55 & 1496.69 & 1499.52 & 1500.30 & 1501.05 \\
\hline 1502.51 & 1502.99 & 1503.67 & 1504.25 & 1505.26 & 1506.65 \\
\hline 1506.80 & 1508.11 & 1510.28 & 1513.33 & 1515.12 & 1515.33 \\
\hline 1518.82 & 1521.21 & 1522.27 & 1523.87 & 1524.07 & 1525.82 \\
\hline 1530.55 & 1533.29 & 1533.48 & 1538.42 & 1542.96 & 1544.98 \\
\hline 1545.59 & 1550.24 & 1552.87 & 1643.28 & 1657.45 & 1699.15 \\
\hline 1764.20 & 2994.36 & 3006.74 & 3009.89 & 3015.18 & 3019.26 \\
\hline 3020.01 & 3021.32 & 3023.12 & 3023.70 & 3025.55 & 3027.25 \\
\hline 3029.21 & 3031.50 & 3031.75 & 3035.66 & 3035.95 & 3036.94 \\
\hline 3037.48 & 3038.36 & 3042.46 & 3042.54 & 3046.28 & 3049.71 \\
\hline 3057.42 & 3067.61 & 3086.49 & 3090.85 & 3101.46 & 3102.82 \\
\hline
\end{tabular}

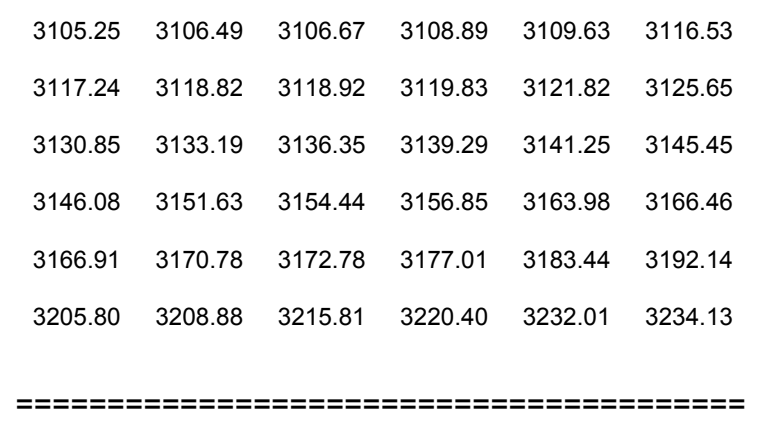

C1

\begin{tabular}{|c|c|c|c|c|c|}
\hline 18.56 & 25.61 & 32.87 & 39.38 & 41.43 & 44.20 \\
\hline 49.24 & 51.41 & 53.80 & 56.04 & 60.40 & 65.62 \\
\hline 67.24 & 81.94 & 92.81 & 99.87 & 109.29 & 113.58 \\
\hline 122.72 & 131.35 & 133.45 & 137.38 & 145.88 & 147.39 \\
\hline 180.88 & 188.51 & 194.52 & 203.92 & 204.81 & 212.52 \\
\hline 214.72 & 218.12 & 223.42 & 227.47 & 230.70 & 235.01 \\
\hline 237.74 & 251.72 & 255.49 & 261.12 & 263.87 & 267.96 \\
\hline 273.54 & 277.13 & 277.23 & 281.03 & 282.14 & 289.34 \\
\hline 294.14 & 296.06 & 299.96 & 301.28 & 304.39 & 309.41 \\
\hline 324.17 & 334.49 & 342.50 & 359.56 & 360.14 & 363.86 \\
\hline 368.82 & 373.24 & 379.24 & 386.05 & 398.25 & 401.32 \\
\hline 410.07 & 418.51 & 432.51 & 434.30 & 435.67 & 439.31 \\
\hline 446.90 & 454.40 & 458.73 & 467.60 & 473.22 & 482.32 \\
\hline 486.82 & 488.53 & 510.43 & 520.76 & 529.94 & 577.35 \\
\hline 599.42 & 608.29 & 620.59 & 631.19 & 636.85 & 689.24 \\
\hline 708.40 & 739.46 & 758.84 & 765.52 & 776.38 & 782.33 \\
\hline 794.84 & 802.69 & 806.44 & 815.51 & 816.90 & 819.49 \\
\hline 839.16 & 842.52 & 847.18 & 874.55 & 898.82 & 909.80 \\
\hline 910.32 & 931.36 & 933.19 & 937.11 & 937.36 & 937.97 \\
\hline 940.08 & 942.85 & 947.47 & 949.42 & 950.72 & 952.20 \\
\hline 954.26 & 962.67 & 976.02 & 979.38 & 980.63 & 981.88 \\
\hline 986.43 & 987.09 & 997.98 & 1016.30 & 1024.28 & 1036.30 \\
\hline 1038.26 & 1046.29 & 1048.14 & 1054.30 & 1054.63 & 1056.62 \\
\hline 1057.88 & 1063.59 & 1063.92 & 1066.95 & 1068.72 & 1071.37 \\
\hline 1077.70 & 1119.22 & 1147.37 & 1189.36 & 1194.69 & 1202.54 \\
\hline 1204.26 & 1205.45 & 1206.47 & 1211.07 & 1215.27 & 1219.75 \\
\hline 1221.38 & 1222.64 & 1223.38 & 1233.64 & 1234.14 & 1235.85 \\
\hline 1238.81 & 1259.70 & 1273.65 & 1277.65 & 1279.49 & 1286.87 \\
\hline 1297.18 & 1315.27 & 1321.48 & 1331.86 & 1347.17 & 1349.42 \\
\hline 1365.85 & 1375.42 & 1409.16 & 1412.24 & 1412.70 & 1413.60 \\
\hline 1416.48 & 1417.55 & 1418.18 & 1422.71 & 1423.86 & 1424.32 \\
\hline 1437.13 & 1442.41 & 1449.26 & 1450.51 & 1461.47 & 1482.46 \\
\hline 1483.79 & 1488.81 & 1491.29 & 1492.18 & 1494.57 & 1496.12 \\
\hline
\end{tabular}




\begin{tabular}{|c|c|c|c|c|c|c|c|c|c|c|c|}
\hline 1498.44 & 1499.70 & 1500.55 & 1502.22 & 1503.23 & 1504.49 & 1082.93 & 1123.47 & 1151.68 & 1196.82 & 1198.57 & 1204.22 \\
\hline 1505.78 & 1507.02 & 1507.32 & 1508.25 & 1509.90 & 1512.30 & 1204.61 & 1206.97 & 1207.79 & 1210.32 & 1214.21 & 1219.41 \\
\hline 1514.33 & 1514.73 & 1519.71 & 1521.22 & 1521.72 & 1523.21 & 1221.15 & 1221.89 & 1224.98 & 1234.50 & 1235.45 & 1235.57 \\
\hline 1525.09 & 1530.73 & 1531.63 & 1535.39 & 1542.19 & 1543.25 & 1239.50 & 1263.56 & 1275.51 & 1276.69 & 1277.27 & 1286.55 \\
\hline 1544.28 & 1547.63 & 1547.74 & 1548.22 & 1641.36 & 1653.97 & 1296.42 & 1311.73 & 1326.55 & 1327.69 & 1343.78 & 1353.37 \\
\hline 1658.57 & 1757.90 & 1775.52 & 3019.24 & 3023.67 & 3035.25 & 1366.14 & 1373.80 & 1409.47 & 1412.10 & 1412.32 & 1413.50 \\
\hline 3037.32 & 3037.97 & 3040.32 & 3040.68 & 3041.48 & 3042.78 & 1414.08 & 1414.89 & 1416.31 & 1418.75 & 1419.86 & 1421.27 \\
\hline 3043.68 & 3043.93 & 3045.47 & 3045.97 & 3048.33 & 3049.63 & 1439.05 & 1440.28 & 1444.95 & 1446.61 & 1457.03 & 1481.66 \\
\hline 3050.83 & 3052.34 & 3056.95 & 3063.94 & 3072.04 & 3081.83 & 1482.54 & 1487.66 & 1488.99 & 1490.36 & 1493.49 & 1496.55 \\
\hline 3099.91 & 3105.02 & 3107.19 & 3109.32 & 3109.45 & 3112.15 & 1498.59 & 1499.01 & 1499.40 & 1501.55 & 1501.91 & 1502.37 \\
\hline 3113.76 & 3116.25 & 3117.21 & 3118.42 & 3123.14 & 3126.04 & 1503.02 & 1505.66 & 1506.39 & 1507.91 & 1508.36 & 1511.61 \\
\hline 3126.57 & 3129.72 & 3131.21 & 3138.15 & 3144.36 & 3147.68 & 1512.11 & 1515.90 & 1519.49 & 1519.87 & 1521.05 & 1521.65 \\
\hline 3150.69 & 3151.59 & 3154.40 & 3157.99 & 3159.27 & 3160.94 & 1522.23 & 1529.72 & 1531.29 & 1534.29 & 1543.15 & 1543.16 \\
\hline 3164.13 & 3170.22 & 3170.94 & 3172.55 & 3181.92 & 3183.72 & 1543.71 & 1544.38 & 1545.79 & 1548.05 & 1585.71 & 1644.11 \\
\hline 3191.52 & 3198.83 & 3203.28 & 3221.61 & 3228.20 & 3242.26 & 1658.87 & 1765.92 & 1786.45 & 2993.16 & 3027.62 & 3030.31 \\
\hline ニニニニニニ & =ニニニニ= & ニニニニニ= & E=ニニニニ= & ニニニニニニ & :=ニ=ニ= & 3032.32 & 3033.84 & 3034.61 & 3035.17 & 3037.76 & 3039.02 \\
\hline C1-TS & & & & & & 3041.57 & 3045.64 & 3046.51 & 3046.57 & 3047.64 & 3050.10 \\
\hline$====ニ=$ & =ニニニニ= & $===ニ==$ & $=====ニ=$ & $====ニ=$ & :=ニ=ニ=ニ & 3051.32 & 3054.25 & 3058.25 & 3058.43 & 3090.84 & 3099.08 \\
\hline-338.01 & 14.16 & 15.96 & 27.89 & 33.38 & 35.01 & 3100.41 & 3101.80 & 3102.45 & 3105.62 & 3110.69 & 3113.34 \\
\hline 47.14 & 48.87 & 52.00 & 58.37 & 66.17 & 67.13 & 3113.69 & 3114.70 & 3117.60 & 3118.35 & 3118.99 & 3121.57 \\
\hline 69.30 & 84.76 & 88.20 & 102.78 & 103.35 & 115.39 & 3128.38 & 3130.59 & 3130.72 & 3143.40 & 3145.22 & 3145.61 \\
\hline 117.48 & 121.62 & 126.00 & 132.79 & 134.59 & 142.89 & 3147.60 & 3150.12 & 3151.21 & 3155.83 & 3158.08 & 3162.66 \\
\hline 148.16 & 186.32 & 192.64 & 196.05 & 201.74 & 206.30 & 3166.59 & 3168.26 & 3170.25 & 3174.48 & 3175.65 & 3179.80 \\
\hline 217.15 & 220.60 & 225.02 & 230.76 & 232.43 & 233.18 & 3184.70 & 3191.22 & 3200.13 & 3229.76 & 3236.33 & 3246.25 \\
\hline 235.68 & 241.66 & 249.19 & 253.18 & 260.86 & 263.49 & \multicolumn{6}{|c|}{ 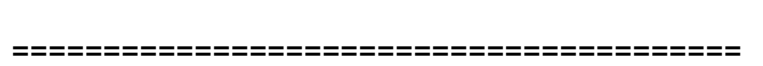 } \\
\hline 264.20 & 271.51 & 274.29 & 276.36 & 281.43 & 282.18 & \multicolumn{6}{|c|}{$\mathrm{C2}$} \\
\hline 288.39 & 294.60 & 296.42 & 299.44 & 301.93 & 303.05 & \multicolumn{6}{|c|}{ 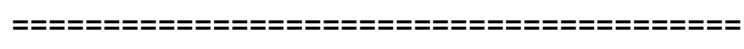 } \\
\hline 323.40 & 334.82 & 341.58 & 358.50 & 360.97 & 364.54 & 11.80 & 19.11 & 26.84 & 32.47 & 37.77 & 39.45 \\
\hline 368.52 & 372.36 & 374.80 & 379.02 & 384.97 & 398.15 & 44.01 & 46.32 & 49.35 & 53.21 & 53.75 & 59.13 \\
\hline 399.40 & 419.46 & 431.27 & 432.28 & 432.74 & 438.60 & 66.03 & 79.49 & 83.26 & 90.74 & 97.15 & 106.99 \\
\hline 448.01 & 453.54 & 462.30 & 467.11 & 470.55 & 481.17 & 118.08 & 128.37 & 129.85 & 137.58 & 141.75 & 145.61 \\
\hline 486.16 & 486.64 & 509.70 & 518.21 & 531.47 & 575.89 & 159.13 & 183.69 & 189.44 & 198.00 & 204.08 & 207.02 \\
\hline 596.12 & 598.92 & 609.03 & 631.21 & 641.81 & 694.79 & 211.10 & 217.10 & 220.98 & 228.32 & 232.49 & 237.63 \\
\hline 712.52 & 746.00 & 749.71 & 757.84 & 765.80 & 772.22 & 239.24 & 242.56 & 249.67 & 254.16 & 262.05 & 263.36 \\
\hline 777.02 & 778.84 & 802.96 & 816.60 & 817.79 & 818.67 & 265.21 & 273.36 & 275.34 & 278.23 & 279.65 & 280.74 \\
\hline 840.11 & 842.36 & 844.85 & 876.91 & 898.97 & 914.40 & 293.92 & 298.39 & 301.04 & 301.72 & 302.72 & 312.85 \\
\hline 918.20 & 926.90 & 933.92 & 935.60 & 937.79 & 938.17 & 316.88 & 325.15 & 334.83 & 342.95 & 355.32 & 360.64 \\
\hline 938.63 & 940.14 & 941.90 & 951.01 & 952.41 & 953.54 & 364.72 & 368.04 & 368.48 & 373.94 & 384.61 & 397.30 \\
\hline 961.04 & 964.28 & 976.96 & 978.36 & 980.41 & 982.31 & 399.31 & 404.32 & 416.29 & 431.28 & 433.68 & 434.41 \\
\hline 983.07 & 983.92 & 985.68 & 1016.55 & 1018.72 & 1038.24 & 436.09 & 442.91 & 448.31 & 457.46 & 466.83 & 473.27 \\
\hline 1038.84 & 1045.43 & 1047.38 & 1053.62 & 1054.35 & 1055.54 & 480.91 & 485.57 & 485.86 & 490.85 & 506.73 & 515.88 \\
\hline 1057.09 & 1064.30 & 1065.41 & 1065.96 & 1066.18 & 1070.84 & 523.67 & 530.05 & 575.88 & 596.77 & 605.00 & 630.74 \\
\hline
\end{tabular}




\begin{tabular}{|c|c|c|c|c|c|c|c|c|c|c|c|}
\hline 637.63 & 687.78 & 706.18 & 721.11 & 741.06 & 765.70 & 147.73 & 179.67 & 196.03 & 199.74 & 203.21 & 207.64 \\
\hline 769.60 & 777.56 & 781.11 & 805.50 & 808.66 & 814.31 & 212.72 & 218.79 & 220.16 & 225.52 & 227.76 & 228.32 \\
\hline 816.41 & 820.81 & 837.37 & 840.09 & 843.89 & 846.37 & 229.37 & 237.90 & 244.36 & 249.95 & 261.47 & 262.44 \\
\hline 876.63 & 907.82 & 908.84 & 920.38 & 931.60 & 934.04 & 267.94 & 269.75 & 273.49 & 278.20 & 279.79 & 281.65 \\
\hline 936.92 & 937.29 & 937.82 & 939.66 & 940.17 & 944.19 & 285.26 & 289.70 & 292.49 & 295.62 & 301.79 & 306.76 \\
\hline 946.71 & 950.42 & 952.53 & 953.57 & 961.68 & 973.27 & 311.99 & 326.32 & 335.62 & 343.30 & 353.57 & 365.08 \\
\hline 978.79 & 980.93 & 983.65 & 984.93 & 985.60 & 1008.28 & 365.26 & 367.43 & 370.74 & 374.77 & 383.55 & 397.78 \\
\hline 1016.20 & 1030.63 & 1036.82 & 1045.48 & 1046.21 & 1047.36 & 399.78 & 400.41 & 416.18 & 431.64 & 434.35 & 435.56 \\
\hline 1054.09 & 1056.44 & 1057.31 & 1057.63 & 1063.18 & 1063.81 & 439.59 & 442.39 & 450.20 & 459.54 & 468.81 & 471.42 \\
\hline 1066.48 & 1072.97 & 1083.21 & 1085.66 & 1093.70 & 1119.84 & 475.35 & 483.28 & 484.81 & 486.95 & 508.56 & 519.47 \\
\hline 1162.75 & 1191.32 & 1192.67 & 1195.28 & 1202.39 & 1205.34 & 531.89 & 539.03 & 575.05 & 593.86 & 596.71 & 630.86 \\
\hline 1208.04 & 1210.55 & 1214.42 & 1220.56 & 1220.90 & 1222.23 & 640.85 & 691.68 & 692.75 & 708.89 & 745.87 & 758.24 \\
\hline 1223.19 & 1231.90 & 1235.88 & 1238.59 & 1266.28 & 1273.47 & 765.08 & 777.12 & 779.36 & 798.00 & 806.48 & 816.28 \\
\hline 1278.64 & 1279.89 & 1284.81 & 1287.12 & 1297.94 & 1314.10 & 818.64 & 819.87 & 824.65 & 830.84 & 840.75 & 845.79 \\
\hline 1339.06 & 1346.59 & 1352.94 & 1361.84 & 1366.01 & 1374.98 & 876.95 & 889.08 & 906.23 & 912.99 & 920.14 & 934.32 \\
\hline 1382.37 & 1387.70 & 1407.90 & 1409.80 & 1411.91 & 1412.04 & 937.35 & 937.75 & 938.43 & 938.78 & 939.05 & 940.59 \\
\hline 1414.53 & 1416.34 & 1417.99 & 1421.14 & 1422.10 & 1423.55 & 941.50 & 950.87 & 953.85 & 955.28 & 959.93 & 976.66 \\
\hline 1436.34 & 1441.86 & 1446.79 & 1449.20 & 1459.79 & 1481.76 & 980.77 & 983.01 & 985.59 & 986.05 & 988.03 & 991.91 \\
\hline 1482.83 & 1483.45 & 1489.73 & 1490.89 & 1491.76 & 1493.89 & 1012.96 & 1016.90 & 1039.01 & 1045.64 & 1046.38 & 1047.78 \\
\hline 1497.77 & 1498.58 & 1499.13 & 1500.72 & 1501.67 & 1503.67 & 1049.92 & 1054.63 & 1055.77 & 1057.36 & 1064.41 & 1066.65 \\
\hline 1504.21 & 1505.74 & 1506.25 & 1508.67 & 1509.24 & 1509.72 & 1068.11 & 1071.79 & 1081.30 & 1095.52 & 1096.08 & 1125.74 \\
\hline 1511.47 & 1512.04 & 1512.73 & 1520.10 & 1520.68 & 1520.92 & 1164.69 & 1189.05 & 1197.33 & 1199.34 & 1203.57 & 1205.19 \\
\hline 1522.93 & 1524.18 & 1529.92 & 1531.12 & 1534.40 & 1541.52 & 1206.75 & 1210.32 & 1213.64 & 1217.80 & 1220.71 & 1220.97 \\
\hline 1542.26 & 1544.50 & 1545.64 & 1547.62 & 1548.12 & 1640.93 & 1227.26 & 1234.44 & 1236.39 & 1239.83 & 1272.44 & 1272.85 \\
\hline 1657.09 & 1694.69 & 1741.75 & 1780.24 & 2998.90 & 3016.79 & 1277.01 & 1279.51 & 1281.20 & 1290.54 & 1294.27 & 1302.70 \\
\hline 3021.79 & 3033.41 & 3034.19 & 3037.05 & 3037.85 & 3038.03 & 1323.17 & 1336.96 & 1355.48 & 1361.57 & 1366.78 & 1371.48 \\
\hline 3038.94 & 3039.85 & 3042.19 & 3042.19 & 3042.67 & 3043.74 & 1385.19 & 1391.89 & 1409.18 & 1412.13 & 1412.84 & 1414.20 \\
\hline 3044.24 & 3045.03 & 3047.91 & 3049.14 & 3052.02 & 3055.37 & 1414.77 & 1416.08 & 1416.45 & 1418.63 & 1420.17 & 1422.62 \\
\hline 3071.15 & 3088.76 & 3092.28 & 3100.29 & 3104.10 & 3108.12 & 1440.14 & 1441.22 & 1445.27 & 1447.65 & 1457.12 & 1481.51 \\
\hline 3110.45 & 3112.18 & 3113.56 & 3114.90 & 3117.17 & 3117.58 & 1482.59 & 1484.28 & 1487.29 & 1489.03 & 1490.21 & 1494.29 \\
\hline 3122.86 & 3124.95 & 3126.01 & 3126.40 & 3129.07 & 3132.19 & 1497.91 & 1499.02 & 1500.52 & 1501.24 & 1502.71 & 1503.37 \\
\hline 3134.86 & 3144.83 & 3146.00 & 3151.82 & 3153.74 & 3156.64 & 1503.66 & 1503.86 & 1505.71 & 1507.68 & 1508.25 & 1509.45 \\
\hline 3160.13 & 3160.87 & 3161.08 & 3165.22 & 3167.47 & 3168.12 & 1511.69 & 1512.61 & 1515.53 & 1519.19 & 1520.60 & 1521.04 \\
\hline 3170.63 & 3171.13 & 3179.55 & 3180.63 & 3193.22 & 3197.45 & 1522.71 & 1522.98 & 1530.18 & 1530.72 & 1535.05 & 1543.14 \\
\hline 3197.92 & 3232.51 & 3237.03 & & & & 1543.67 & 1544.12 & 1544.56 & 1545.54 & 1549.25 & 1644.38 \\
\hline \multicolumn{6}{|c|}{ 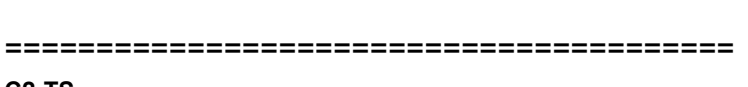 } & 1653.05 & 1659.68 & 1758.29 & 1794.21 & 2979.83 & 3019.74 \\
\hline C2-TS & & & & & & 3029.0 & 3029.96 & 3032.60 & 3033.78 & 3034.24 & 3034.52 \\
\hline \multicolumn{6}{|c|}{ 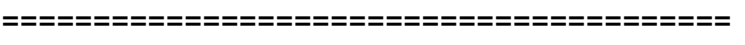 } & 3035.12 & 3036.03 & 3039.66 & 3040.58 & 3043.46 & 3047.01 \\
\hline-327.21 & 17.04 & 21.59 & 27.80 & 38.24 & 41.74 & 3047.95 & 3047.97 & 3050.32 & 3050.63 & 3056.87 & 3057.88 \\
\hline 43.86 & 48.47 & 49.55 & 60.93 & 63.08 & 67.51 & 3073.82 & 3098.92 & 3100.68 & 3103.08 & 3103.12 & 3104.19 \\
\hline 73.11 & 83.74 & 93.32 & 101.57 & 107.28 & 111.06 & 3111.49 & 3113.84 & 3114.21 & 3114.24 & 3114.45 & 3118.91 \\
\hline 115.07 & 119.72 & 124.66 & 130.00 & 133.91 & 142.85 & 3119.77 & 3126.35 & 3127.37 & 3129.97 & 3132.97 & 3135.48 \\
\hline
\end{tabular}




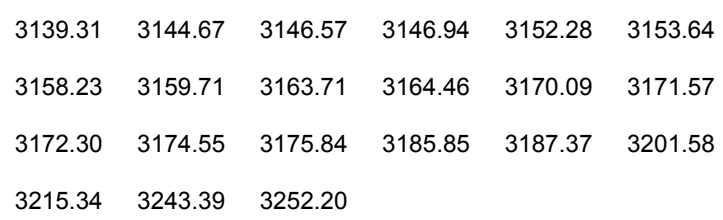

\begin{tabular}{|c|c|c|c|c|c|}
\hline 15.59 & 22.29 & 25.34 & 29.04 & 33.87 & 37.24 \\
\hline 41.07 & 46.19 & 49.87 & 52.17 & 57.27 & 62.73 \\
\hline 65.38 & 75.79 & 84.28 & 87.43 & 103.93 & 107.69 \\
\hline 115.15 & 117.87 & 126.86 & 134.64 & 136.75 & 143.56 \\
\hline 146.05 & 169.83 & 175.26 & 187.57 & 190.97 & 200.48 \\
\hline 201.48 & 209.18 & 213.41 & 219.84 & 230.90 & 231.83 \\
\hline 236.51 & 238.04 & 243.96 & 253.18 & 260.01 & 262.06 \\
\hline 263.63 & 265.39 & 272.28 & 277.13 & 279.76 & 281.74 \\
\hline 283.09 & 288.72 & 295.37 & 296.92 & 299.68 & 303.31 \\
\hline 305.78 & 307.01 & 327.74 & 334.58 & 341.22 & 352.57 \\
\hline 357.90 & 359.81 & 361.28 & 366.70 & 368.85 & 374.89 \\
\hline 385.39 & 398.67 & 401.95 & 405.29 & 417.43 & 421.39 \\
\hline 428.40 & 433.62 & 435.64 & 443.96 & 447.78 & 454.52 \\
\hline 460.37 & 467.04 & 473.88 & 481.47 & 487.04 & 489.32 \\
\hline 505.68 & 518.11 & 529.73 & 570.31 & 575.33 & 598.29 \\
\hline 604.21 & 630.96 & 638.14 & 689.63 & 701.11 & 706.63 \\
\hline 741.08 & 764.63 & 767.26 & 774.74 & 776.95 & 780.08 \\
\hline 788.00 & 807.30 & 811.93 & 817.20 & 817.87 & 835.68 \\
\hline 838.35 & 844.86 & 867.40 & 876.33 & 882.46 & 908.71 \\
\hline 931.32 & 933.47 & 934.93 & 938.10 & 938.56 & 940.08 \\
\hline 941.32 & 943.30 & 948.87 & 951.42 & 953.74 & 955.40 \\
\hline 961.67 & 966.48 & 976.31 & 979.01 & 979.16 & 983.90 \\
\hline 984.32 & 986.57 & 998.37 & 1016.01 & 1027.31 & 1028.66 \\
\hline 1038.90 & 1045.23 & 1046.96 & 1053.14 & 1054.10 & 1055.19 \\
\hline 1057.67 & 1058.47 & 1063.46 & 1064.91 & 1067.11 & 1072.76 \\
\hline 1082.66 & 1092.70 & 1119.73 & 1130.41 & 1173.47 & 1194.67 \\
\hline 1195.40 & 1200.90 & 1205.26 & 1208.14 & 1210.33 & 1213.14 \\
\hline 1219.69 & 1220.50 & 1221.04 & 1223.83 & 1225.65 & 1232.44 \\
\hline 1234.15 & 1238.27 & 1248.22 & 1273.77 & 1279.26 & 1279.70 \\
\hline 1281.56 & 1286.87 & 1298.13 & 1308.28 & 1329.61 & 1338.85 \\
\hline 1349.54 & 1360.38 & 1366.28 & 1367.37 & 1376.55 & 1381.33 \\
\hline 1399.08 & 1406.97 & 1408.69 & 1411.21 & 1412.34 & 1414.46 \\
\hline 1417.88 & 1418.52 & 1419.13 & 1421.77 & 1426.85 & 1428.20 \\
\hline 1435.78 & 1442.21 & 1449.61 & 1453.77 & 1462.94 & 1479.56 \\
\hline 1482.73 & 1484.25 & 1484.99 & 1489.98 & 1490.51 & 1492.99 \\
\hline
\end{tabular}

\begin{tabular}{|c|c|c|c|c|c|}
\hline 1495.48 & 1496.10 & 1497.68 & 1500.44 & 1500.56 & 1502.13 \\
\hline 1503.14 & 1504.77 & 1506.50 & 1506.85 & 1508.17 & 1510.09 \\
\hline 1510.87 & 1512.29 & 1513.10 & 1518.90 & 1520.29 & 1520.63 \\
\hline 1521.77 & 1522.66 & 1524.02 & 1530.07 & 1531.07 & 1535.06 \\
\hline 1541.96 & 1542.20 & 1544.10 & 1544.77 & 1547.90 & 1548.68 \\
\hline 1640.53 & 1657.04 & 1691.52 & 1754.52 & 1779.12 & 2988.75 \\
\hline 3017.50 & 3028.56 & 3029.32 & 3036.53 & 3037.42 & 3038.01 \\
\hline 3039.55 & 3040.33 & 3041.16 & 3041.81 & 3041.89 & 3043.31 \\
\hline 3043.45 & 3045.92 & 3049.23 & 3050.04 & 3050.27 & 3050.74 \\
\hline 3053.46 & 3057.90 & 3058.19 & 3074.02 & 3084.83 & 3094.72 \\
\hline 3101.94 & 3102.30 & 3105.03 & 3109.04 & 3109.36 & 3112.39 \\
\hline 3113.57 & 3115.22 & 3115.73 & 3120.91 & 3121.29 & 3125.63 \\
\hline 3126.08 & 3126.34 & 3133.88 & 3142.52 & 3144.25 & 3145.26 \\
\hline 3147.01 & 3147.95 & 3152.48 & 3153.93 & 3158.36 & 3160.68 \\
\hline 3164.53 & 3164.63 & 3167.47 & 3168.22 & 3173.12 & 3182.34 \\
\hline 3182.77 & 3188.15 & 3188.33 & 3199.20 & 3238.89 & 3240.59 \\
\hline \multirow{2}{*}{\multicolumn{6}{|c|}{ 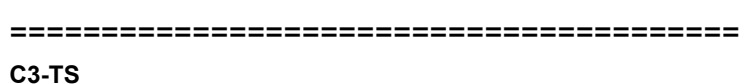 }} \\
\hline C3-TS & & & & & \\
\hline \multicolumn{6}{|c|}{ 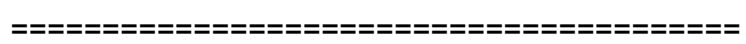 } \\
\hline-289.08 & 18.16 & 24.18 & 30.08 & 36.50 & 37.15 \\
\hline 40.99 & 51.32 & 53.40 & 60.35 & 62.33 & 67.52 \\
\hline 74.04 & 75.07 & 87.80 & 90.93 & 98.88 & 108.62 \\
\hline 112.25 & 117.29 & 122.30 & 131.21 & 135.56 & 143.52 \\
\hline 154.76 & 166.24 & 179.76 & 192.91 & 196.63 & 202.72 \\
\hline 204.70 & 209.72 & 210.09 & 218.62 & 225.56 & 228.17 \\
\hline 234.49 & 237.72 & 241.38 & 248.87 & 262.50 & 264.54 \\
\hline 268.18 & 270.44 & 273.97 & 274.18 & 281.00 & 282.61 \\
\hline 283.23 & 291.64 & 296.03 & 297.29 & 299.44 & 302.59 \\
\hline 303.33 & 306.38 & 325.16 & 336.08 & 342.79 & 353.73 \\
\hline 356.13 & 366.81 & 367.35 & 367.50 & 372.24 & 376.84 \\
\hline 385.11 & 395.37 & 399.30 & 400.88 & 410.21 & 418.51 \\
\hline 431.18 & 432.47 & 434.25 & 438.54 & 447.56 & 453.77 \\
\hline 454.90 & 469.34 & 470.83 & 482.36 & 485.27 & 487.42 \\
\hline 511.05 & 517.49 & 535.53 & 569.04 & 574.52 & 593.77 \\
\hline 597.50 & 630.14 & 638.64 & 655.84 & 691.34 & 707.88 \\
\hline 745.83 & 759.60 & 764.28 & 769.10 & 775.84 & 778.99 \\
\hline 788.74 & 804.97 & 814.74 & 816.49 & 818.67 & 823.69 \\
\hline 831.44 & 840.61 & 854.65 & 872.99 & 877.84 & 904.86 \\
\hline 921.39 & 930.66 & 934.46 & 937.29 & 937.75 & 938.74 \\
\hline 939.50 & 940.30 & 941.77 & 949.91 & 951.51 & 955.06 \\
\hline 960.93 & 963.34 & 975.50 & 978.22 & 982.41 & 984.81 \\
\hline 985.88 & 986.00 & 999.07 & 1010.80 & 1015.74 & 1027.68 \\
\hline
\end{tabular}




\begin{tabular}{|c|c|c|c|c|c|}
\hline 1039.08 & 1045.82 & 1046.38 & 1046.60 & 1054.19 & 1055.03 \\
\hline 1056.74 & 1064.25 & 1064.90 & 1065.50 & 1067.48 & 1071.45 \\
\hline 1079.42 & 1095.12 & 1125.71 & 1127.12 & 1175.22 & 1196.01 \\
\hline 1198.15 & 1200.12 & 1203.52 & 1205.70 & 1209.83 & 1211.96 \\
\hline 1215.08 & 1219.76 & 1220.75 & 1221.53 & 1228.34 & 1233.87 \\
\hline 1235.06 & 1239.51 & 1249.04 & 1268.83 & 1274.91 & 1277.14 \\
\hline 1279.73 & 1284.61 & 1292.19 & 1306.32 & 1308.57 & 1328.96 \\
\hline 1336.34 & 1356.29 & 1361.33 & 1370.95 & 1371.78 & 1380.76 \\
\hline 1395.77 & 1403.29 & 1410.02 & 1411.75 & 1413.32 & 1413.72 \\
\hline 1414.81 & 1415.14 & 1417.56 & 1418.56 & 1420.05 & 1422.45 \\
\hline 1439.31 & 1441.72 & 1445.49 & 1447.04 & 1457.05 & 1482.49 \\
\hline 1483.56 & 1485.63 & 1489.13 & 1489.55 & 1492.24 & 1493.05 \\
\hline 1497.26 & 1497.84 & 1498.78 & 1499.89 & 1501.60 & 1502.67 \\
\hline 1503.71 & 1504.41 & 1507.15 & 1508.08 & 1508.79 & 1509.71 \\
\hline 1511.32 & 1512.25 & 1515.93 & 1518.44 & 1520.41 & 1521.35 \\
\hline 1521.93 & 1522.55 & 1523.36 & 1530.07 & 1530.45 & 1534.29 \\
\hline 1541.50 & 1543.47 & 1544.73 & 1545.07 & 1546.58 & 1548.50 \\
\hline 1641.56 & 1650.84 & 1658.43 & 1759.07 & 1790.52 & 2974.22 \\
\hline 3020.11 & 3026.10 & 3030.23 & 3032.46 & 3032.85 & 3033.05 \\
\hline 3034.50 & 3035.79 & 3038.26 & 3040.83 & 3043.12 & 3044.04 \\
\hline 3046.44 & 3046.69 & 3047.39 & 3048.14 & 3054.03 & 3054.95 \\
\hline 3056.18 & 3062.19 & 3065.78 & 3081.46 & 3096.00 & 3097.88 \\
\hline 3102.69 & 3103.24 & 3104.15 & 3107.34 & 3112.52 & 3113.90 \\
\hline 3114.11 & 3114.58 & 3114.87 & 3117.87 & 3119.76 & 3126.44 \\
\hline 3126.66 & 3128.58 & 3133.26 & 3135.67 & 3144.91 & 3145.46 \\
\hline 3151.61 & 3154.53 & 3156.31 & 3157.78 & 3158.63 & 3163.31 \\
\hline 3165.16 & 3168.35 & 3169.77 & 3171.20 & 3174.65 & 3174.82 \\
\hline 3176.52 & 3184.34 & 3200.80 & 3212.47 & 3256.67 & 3259.79 \\
\hline
\end{tabular}

C
C4

\begin{tabular}{|c|c|c|c|c|c|}
\hline 11.74 & 19.16 & 32.33 & 35.19 & 42.25 & 46.09 \\
\hline 51.58 & 52.79 & 56.77 & 58.60 & 60.70 & 71.27 \\
\hline 74.93 & 80.53 & 82.45 & 90.61 & 96.13 & 104.28 \\
\hline 113.63 & 124.85 & 128.12 & 131.19 & 139.28 & 144.90 \\
\hline 146.84 & 154.78 & 189.83 & 194.35 & 205.90 & 206.87 \\
\hline 210.76 & 214.10 & 217.83 & 222.34 & 227.80 & 231.23 \\
\hline 233.38 & 239.60 & 240.69 & 245.19 & 253.89 & 260.25 \\
\hline 261.77 & 265.19 & 267.67 & 275.26 & 277.11 & 278.24 \\
\hline 279.51 & 280.23 & 293.60 & 294.76 & 299.54 & 301.29 \\
\hline 303.02 & 304.97 & 309.07 & 326.20 & 336.19 & 341.97 \\
\hline 352.98 & 357.60 & 363.64 & 370.64 & 370.93 & 372.05 \\
\hline
\end{tabular}

\begin{tabular}{|c|c|c|c|c|c|}
\hline 373.95 & 380.33 & 386.19 & 397.06 & 400.58 & 402.43 \\
\hline 419.61 & 433.14 & 434.55 & 436.00 & 440.79 & 446.97 \\
\hline 458.49 & 465.44 & 467.65 & 472.61 & 479.46 & 485.58 \\
\hline 487.98 & 506.68 & 518.69 & 525.53 & 534.28 & 575.74 \\
\hline 581.21 & 597.60 & 607.22 & 626.27 & 631.23 & 638.15 \\
\hline 683.81 & 710.78 & 739.37 & 743.61 & 764.48 & 773.76 \\
\hline 775.09 & 785.97 & 789.76 & 809.02 & 814.86 & 818.29 \\
\hline 819.32 & 831.68 & 841.44 & 842.55 & 852.77 & 873.03 \\
\hline 874.32 & 898.72 & 911.61 & 926.05 & 931.33 & 933.51 \\
\hline 936.61 & 936.90 & 938.61 & 940.05 & 942.65 & 947.92 \\
\hline 949.00 & 950.21 & 955.10 & 969.04 & 975.23 & 979.45 \\
\hline 980.34 & 982.73 & 985.78 & 988.10 & 993.26 & 1002.97 \\
\hline 1017.28 & 1017.79 & 1020.72 & 1037.33 & 1044.01 & 1046.60 \\
\hline 47.98 & 1054.30 & 056.29 & 1056.75 & 1057.99 & 1059.71 \\
\hline 063.43 & 1066.27 & 072.47 & 1082.49 & 1085 & 21 \\
\hline 11.51 & 1129.49 & 1171.83 & 1186.05 & 1192.89 & 1202.79 \\
\hline 205.24 & 1206.25 & 1209.36 & 1209.91 & 1211.28 & 1214.88 \\
\hline 1219.98 & 1221.82 & 1222.96 & 1231.67 & 1234.52 & 1238.67 \\
\hline 1239.17 & 1273.66 & 1277.81 & 1278.76 & 1280.17 & 1286.02 \\
\hline 1289.84 & 1297.56 & 1316.24 & 1322.87 & 1334.96 & 1337.86 \\
\hline 356.62 & 1360.26 & 1367.07 & 1374.43 & 1381.90 & 1389.89 \\
\hline 406.60 & 1406.6 & 409.26 & 1411.02 & 1412.83 & 41 \\
\hline 415. & 1416.3 & 1418 & 1420.92 & 142 & 1423.64 \\
\hline 37.06 & 1443.02 & 447 & 1449 & 1460.34 & 1478.89 \\
\hline 1482.34 & 1484.11 & 1489.76 & 1491.95 & 1493.92 & 1494.71 \\
\hline 1496.69 & 1498.09 & 1498.60 & 1500.05 & 1501.36 & 1502.51 \\
\hline 1504.70 & 1504.98 & 1506.12 & 1506.63 & 1507.90 & 1508.59 \\
\hline 1510.15 & 1512. & 1513.19 & 1518.45 & 1519.87 & 1521 \\
\hline 521 & 1522. & 152 & 152 & 153 & 153 \\
\hline 39 & 1540. & 154 & 154 & 1547.42 & 1547.92 \\
\hline 1549.24 & 1640 & 1655.50 & 1684.26 & 1731.42 & 1776.07 \\
\hline 2991.46 & 3008.68 & 3012.83 & 3028.48 & 3031.37 & 3033.47 \\
\hline 3034.47 & 3036.77 & 3037.10 & 3037.63 & 3038.80 & 3041.17 \\
\hline 3041.69 & 3043.5 & 3043 & 3043 & 3048.50 & 3049.01 \\
\hline 3050.71 & 3052 . & 305 & 305 & 305 & 307 \\
\hline 307 & 3085 & 30 & 310 & 310 & 3108.90 \\
\hline 3109 & 3111 & 3114.41 & 3116.86 & 3117.56 & 3119.46 \\
\hline & 3125.04 & 3127.79 & 3132.96 & 3139.27 & 3140.50 \\
\hline 3142.90 & 3147.95 & 3150.22 & 3152.12 & 3153.70 & 3156.19 \\
\hline 31 & 31 & 31 & 31 & 31 & 316 \\
\hline & 31 & 31 & 319 & 319 & \\
\hline 3199.61 & 320 & & & & \\
\hline
\end{tabular}




\begin{tabular}{|c|c|c|c|c|c|}
\hline \multicolumn{6}{|l|}{ C4-TS } \\
\hline \multicolumn{6}{|c|}{ 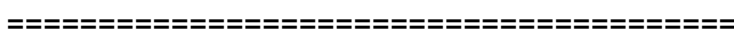 } \\
\hline-260.40 & 12.65 & 21.19 & 28.50 & 36.94 & 44.70 \\
\hline 48.05 & 53.63 & 57.82 & 60.28 & 66.19 & 69.80 \\
\hline 76.16 & 81.32 & 88.89 & 91.55 & 94.63 & 104.98 \\
\hline 113.30 & 117.11 & 123.24 & 131.39 & 135.81 & 138.69 \\
\hline 144.87 & 155.77 & 163.51 & 189.31 & 191.58 & 197.79 \\
\hline 205.08 & 208.65 & 216.75 & 218.05 & 223.62 & 228.85 \\
\hline 230.14 & 231.02 & 234.37 & 241.39 & 247.80 & 253.39 \\
\hline 262.00 & 262.19 & 266.96 & 268.89 & 273.07 & 273.68 \\
\hline 279.84 & 280.37 & 282.62 & 287.19 & 291.67 & 294.70 \\
\hline 296.07 & 302.33 & 304.54 & 325.69 & 335.33 & 343.80 \\
\hline 350.51 & 354.92 & 366.26 & 366.71 & 369.73 & 371.34 \\
\hline 371.64 & 378.95 & 384.60 & 398.85 & 399.53 & 418.16 \\
\hline 423.20 & 431.37 & 433.32 & 435.81 & 436.95 & 439.34 \\
\hline 449.04 & 458.57 & 469.06 & 472.04 & 482.56 & 484.57 \\
\hline 487.15 & 497.63 & 514.03 & 516.52 & 528.57 & 538.77 \\
\hline 573.78 & 593.60 & 596.14 & 605.77 & 630.39 & 639.45 \\
\hline 690.89 & 705.95 & 729.71 & 744.86 & 760.46 & 768.00 \\
\hline 776.86 & 778.86 & 783.19 & 807.11 & 816.06 & 817.75 \\
\hline 819.84 & 821.19 & 826.18 & 831.76 & 840.50 & 853.75 \\
\hline 865.51 & 872.70 & 903.39 & 906.45 & 919.92 & 934.45 \\
\hline 938.29 & 938.51 & 939.28 & 940.00 & 941.38 & 942.14 \\
\hline 952.24 & 954.03 & 954.70 & 962.00 & 978.04 & 978.44 \\
\hline 980.51 & 982.55 & 985.24 & 985.95 & 989.28 & 1007.85 \\
\hline 1010.26 & 1016.76 & 1018.99 & 1039.67 & 1040.98 & 1044.06 \\
\hline 1046.75 & 1047.48 & 1055.20 & 1056.05 & 1056.99 & 1064.02 \\
\hline 1066.99 & 1068.21 & 1071.98 & 1078.80 & 1094.99 & 1113.53 \\
\hline 1120.24 & 1126.11 & 1180.92 & 1195.86 & 1196.40 & 1198.79 \\
\hline 1203.93 & 1205.24 & 1209.86 & 1210.59 & 1214.21 & 1216.21 \\
\hline 1220.73 & 1221.35 & 1228.38 & 1235.45 & 1236.83 & 1240.39 \\
\hline 1250.36 & 1271.25 & 1276.71 & 1280.57 & 1282.13 & 1287.34 \\
\hline 1293.28 & 1296.38 & 1306.93 & 1316.09 & 1326.89 & 1334.24 \\
\hline 1356.77 & 1364.55 & 1370.56 & 1377.84 & 1379.77 & 1390.63 \\
\hline 1404.64 & 1407.77 & 1408.99 & 1411.87 & 1412.45 & 1413.27 \\
\hline 1414.47 & 1415.95 & 1416.44 & 1418.71 & 1420.01 & 1421.34 \\
\hline 1440.09 & 1441.71 & 1445.21 & 1446.47 & 1456.49 & 1465.85 \\
\hline 1482.05 & 1482.85 & 1488.21 & 1489.73 & 1492.64 & 1493.41 \\
\hline 1495.93 & 1498.62 & 1499.00 & 1501.09 & 1501.95 & 1502.38 \\
\hline 1504.13 & 1504.55 & 1505.06 & 1506.38 & 1506.93 & 1508.13 \\
\hline 1508.32 & 1511.42 & 1512.83 & 1513.77 & 1518.74 & 1519.04 \\
\hline
\end{tabular}

\begin{tabular}{llllll}
1519.83 & 1520.66 & 1522.30 & 1523.75 & 1530.01 & 1530.69 \\
1534.67 & 1542.77 & 1543.86 & 1544.50 & 1545.59 & 1546.56 \\
1547.09 & 1642.78 & 1647.37 & 1658.49 & 1749.47 & 1788.89 \\
3011.48 & 3015.91 & 3024.84 & 3029.02 & 3029.40 & 3030.01 \\
3032.82 & 3033.32 & 3034.24 & 3035.12 & 3035.92 & 3038.98 \\
3039.45 & 3041.45 & 3044.51 & 3047.55 & 3047.81 & 3050.11 \\
3051.38 & 3053.95 & 3056.61 & 3060.33 & 3061.26 & 3073.72 \\
3083.81 & 3091.50 & 3097.18 & 3100.72 & 3102.48 & 3105.44 \\
3113.26 & 3113.61 & 3113.75 & 3114.46 & 3115.45 & 3119.24 \\
3120.15 & 3127.30 & 3127.82 & 3134.55 & 3142.93 & 3144.48 \\
3146.63 & 3151.88 & 3153.16 & 3155.51 & 3156.15 & 3160.53 \\
3160.90 & 3162.15 & 3167.12 & 3170.15 & 3171.69 & 3174.07 \\
3174.75 & 3176.21 & 3179.85 & 3186.30 & 3201.34 & 3201.75 \\
3225.82 & 3251.77 & 3256.26 & & & \\
\hline
\end{tabular}

\begin{tabular}{|c|c|c|c|c|c|}
\hline \multicolumn{6}{|l|}{ C5 } \\
\hline$=====$ & $====$ & $:====$ & $=====$ & $=====$ & $======$ \\
\hline 11.17 & 20.75 & 34.34 & 39.22 & 46.75 & 52.36 \\
\hline 54.79 & 55.47 & 56.84 & 62.48 & 70.18 & 74.64 \\
\hline 78.17 & 82.09 & 88.23 & 92.61 & 100.71 & 103.91 \\
\hline 118.90 & 127.71 & 131.67 & 133.47 & 142.52 & 145.27 \\
\hline 150.14 & 152.38 & 159.93 & 182.87 & 190.32 & 198.43 \\
\hline 205.67 & 209.04 & 211.49 & 215.66 & 219.91 & 221.70 \\
\hline 224.54 & 228.15 & 232.65 & 238.86 & 242.79 & 248.24 \\
\hline 257.14 & 259.84 & 265.08 & 272.62 & 275.14 & 277.57 \\
\hline 282.25 & 282.59 & 288.59 & 294.85 & 297.42 & 298.36 \\
\hline 301.21 & 302.11 & 304.88 & 307.22 & 324.85 & 334.97 \\
\hline 340.34 & 351.78 & 356.29 & 366.59 & 367.92 & 370.05 \\
\hline 371.04 & 372.28 & 385.20 & 389.73 & 397.87 & 403.05 \\
\hline 405.75 & 406.92 & 420.96 & 432.93 & 434.37 & 436.83 \\
\hline 439.00 & 445.87 & 460.57 & 469.05 & 472.54 & 474.73 \\
\hline 479.96 & 484.81 & 489.37 & 502.59 & 506.34 & 516.82 \\
\hline 532.40 & 574.38 & 592.77 & 595.51 & 605.42 & 618.05 \\
\hline 631.42 & 638.00 & 683.02 & 710.06 & 717.43 & 735.68 \\
\hline 762.53 & 768.43 & 781.85 & 784.20 & 786.42 & 799.33 \\
\hline 807.88 & 813.62 & 815.08 & 818.94 & 825.41 & 836.44 \\
\hline 840.77 & 851.71 & 868.46 & 871.33 & 923.70 & 926.02 \\
\hline 930.15 & 933.44 & 936.72 & 937.67 & 938.77 & 939.67 \\
\hline 942.40 & 942.62 & 944.75 & 949.80 & 951.29 & 953.30 \\
\hline 966.54 & 970.99 & 977.01 & 978.61 & 980.73 & 985.23 \\
\hline 987.92 & 988.98 & 993.20 & 1018.55 & 1021.73 & 1037.90 \\
\hline 1038.36 & 1044.21 & 1046.38 & 1047.30 & 1054.47 & 1055.80 \\
\hline
\end{tabular}




\begin{tabular}{|c|c|c|c|c|c|c|c|c|c|c|c|}
\hline 1056.90 & 1058.53 & 1062.31 & 1063.78 & 1067.04 & 1070.96 & 346.32 & 349.83 & 354.72 & 365.66 & 367.32 & 370.12 \\
\hline 1073.38 & 1083.14 & 1086.21 & 1110.75 & 1114.63 & 1128.43 & 371.79 & 372.33 & 381.28 & 383.53 & 398.70 & 400.50 \\
\hline 1181.97 & 1186.01 & 1194.27 & 1202.66 & 1203.96 & 1205.39 & 401.91 & 423.23 & 431.07 & 433.39 & 435.10 & 438.56 \\
\hline 1209.74 & 1211.98 & 1214.13 & 1215.45 & 1219.34 & 1220.79 & 440.70 & 447.61 & 454.66 & 468.98 & 471.31 & 473.87 \\
\hline 1221.33 & 1227.69 & 1233.26 & 1234.75 & 1237.42 & 1258.99 & 485.12 & 486.19 & 488.06 & 496.15 & 510.49 & 515.75 \\
\hline 1273.98 & 1276.44 & 1280.19 & 1285.50 & 1290.63 & 1296.24 & 537.08 & 543.13 & 573.78 & 589.55 & 594.03 & 596.39 \\
\hline 1312.23 & 1333.05 & 1337.37 & 1338.42 & 1344.80 & 1355.44 & 629.97 & 640.77 & 690.94 & 705.58 & 718.99 & 742.10 \\
\hline 1362.18 & 1364.75 & 1369.44 & 1375.43 & 1389.37 & 1396.83 & 758.97 & 764.94 & 772.86 & 777.36 & 780.37 & 794.44 \\
\hline 1408.22 & 1411.02 & 1412.11 & 1413.76 & 1414.00 & 1415.36 & 798.07 & 809.18 & 816.68 & 818.43 & 819.87 & 821.13 \\
\hline 1416.58 & 1419.75 & 1420.07 & 1420.80 & 1423.12 & 1426.89 & 837.18 & 840.01 & 852.38 & 872.20 & 911.25 & 918.22 \\
\hline 1437.23 & 1444.86 & 1447.43 & 1450.73 & 1462.01 & 1479.70 & 924.04 & 934.05 & 938.07 & 938.38 & 938.87 & 939.60 \\
\hline 1482.32 & 1485.72 & 1489.78 & 1491.49 & 1492.70 & 1494.14 & 940.30 & 941.91 & 942.73 & 952.38 & 953.82 & 954.51 \\
\hline 1496.30 & 1496.68 & 1499.23 & 1499.69 & 1501.83 & 1503.22 & 964.50 & 969.58 & 978.83 & 979.51 & 981.60 & 982.33 \\
\hline 1503.78 & 1504.01 & 1504.70 & 1506.65 & 1507.31 & 1508.43 & 984.55 & 985.16 & 990.13 & 1006.86 & 1014.16 & 1027.70 \\
\hline 1509.63 & 1512.21 & 1513.48 & 1514.42 & 1515.42 & 1518.85 & 1036.83 & 1037.93 & 1040.10 & 1046.25 & 1047.33 & 1054.92 \\
\hline 1519.65 & 1520.72 & 1522.45 & 1523.24 & 1524.70 & 1530.81 & 1056.20 & 1057.11 & 1064.16 & 1068.10 & 1069.32 & 1071.94 \\
\hline 1533.22 & 1536.33 & 1542.23 & 1544.31 & 1544.87 & 1547.56 & 1077.22 & 1080.30 & 1092.57 & 1112.40 & 1124.53 & 1127.52 \\
\hline 1548.84 & 1549.97 & 1644.51 & 1658.09 & 1688.14 & 1730.04 & 1182.29 & 1187.99 & 1193.63 & 1198.95 & 1203.30 & 1204.63 \\
\hline 1775.69 & 3001.93 & 3015.99 & 3017.69 & 3022.73 & 3030.02 & 1208.27 & 1210.15 & 1214.46 & 1218.40 & 1220.95 & 1221.86 \\
\hline 3033.13 & 3033.22 & 3034.58 & 3037.64 & 3038.59 & 3039.36 & 1230.69 & 1232.56 & 1235.76 & 1237.76 & 1241.06 & 1256.42 \\
\hline 3040.97 & 3041.13 & 3042.88 & 3044.89 & 3044.97 & 3047.75 & 1257.11 & 1276.23 & 1278.23 & 1281.89 & 1288.80 & 1296.19 \\
\hline 3048.16 & 3048.92 & 3053.38 & 3053.43 & 3054.39 & 3057.83 & 1300.41 & 1311.59 & 1318.08 & 1334.94 & 1341.03 & 1354.26 \\
\hline 3058.57 & 3061.69 & 3069.63 & 3077.39 & 3094.26 & 3100.72 & 1360.39 & 1362.41 & 1368.10 & 1370.84 & 1386.13 & 1396.80 \\
\hline 3104.24 & 3109.56 & 3111.32 & 3113.56 & 3114.09 & 3115.66 & 1406.96 & 1409.17 & 1412.00 & 1412.58 & 1413.08 & 1414.93 \\
\hline 3117.44 & 3118.03 & 3119.25 & 3122.60 & 3124.71 & 3126.92 & 1416.27 & 1416.36 & 1419.01 & 1419.80 & 1421.00 & 1421.71 \\
\hline 3129.43 & 3136.61 & 3147.13 & 3148.31 & 3150.16 & 3152.30 & 1440.26 & 1441.91 & 1445.36 & 1446.93 & 1456.75 & 1462.80 \\
\hline 3153.65 & 3156.01 & 3158.50 & 3159.68 & 3160.04 & 3161.88 & 1482.52 & 1482.93 & 1489.03 & 1490.14 & 1492.04 & 1495.35 \\
\hline 3164.05 & 3164.34 & 3172.38 & 3173.61 & 3173.85 & 3176.68 & 1498.43 & 1498.92 & 1499.37 & 1500.59 & 1501.20 & 1502.47 \\
\hline 3183.80 & 3184.13 & 3198.59 & 3201.05 & 3221.57 & 3229.25 & 1504.58 & 1505.03 & 1505.85 & 1506.18 & 1506.22 & 1508.73 \\
\hline \multicolumn{6}{|c|}{ 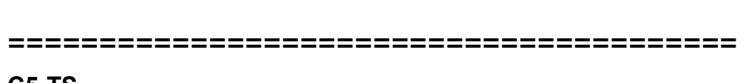 } & 1509.69 & 1511.45 & 1511.63 & 1512.61 & 1512.98 & 1518.50 \\
\hline C5-TS & & & & & & 1519.52 & 1519.82 & 1520.40 & 1522.55 & 1523.84 & 1530.04 \\
\hline \multicolumn{6}{|c|}{ 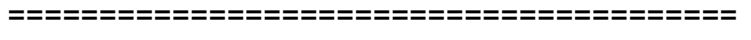 } & 1530.51 & 1534.87 & 1540.11 & 1544.14 & 1544.91 & 1545.37 \\
\hline-262.00 & 15.29 & 19.12 & 28.03 & 36.36 & 46.61 & 1547.10 & 1547.46 & 1640.50 & 1643.67 & 1658.59 & 1751.05 \\
\hline 50.85 & 53.68 & 59.46 & 65.35 & 66.53 & 73.93 & 1787.69 & 3008.63 & 3016.81 & 3018.86 & 3023.38 & 3029.17 \\
\hline 78.24 & 81.27 & 85.09 & 88.60 & 94.06 & 101.18 & 3029.54 & 3029.84 & 3032.98 & 3033.45 & 3033.63 & 3034.75 \\
\hline 109.77 & 116.29 & 126.71 & 129.45 & 135.94 & 141.82 & 3036.58 & 3039.27 & 3042.10 & 3044.93 & 3045.25 & 3047.42 \\
\hline 143.15 & 147.48 & 158.03 & 164.30 & 182.94 & 192.64 & 3047.54 & 3052.02 & 3054.98 & 3058.12 & 3058.45 & 3061.27 \\
\hline 196.90 & 200.74 & 206.21 & 212.65 & 215.33 & 220.87 & 3061.30 & 3067.81 & 3072.92 & 3073.82 & 3097.07 & 3101.69 \\
\hline 224.36 & 228.82 & 231.34 & 233.11 & 235.39 & 248.12 & 3102.42 & 3108.21 & 3113.02 & 3113.34 & 3113.46 & 3114.93 \\
\hline 253.42 & 261.18 & 263.30 & 266.09 & 268.34 & 273.83 & 3115.00 & 3115.75 & 3120.01 & 3121.00 & 3127.44 & 3128.15 \\
\hline 276.09 & 280.77 & 283.43 & 285.09 & 287.13 & 290.31 & 3135.59 & 3141.66 & 3143.45 & 3146.54 & 3150.93 & 3152.85 \\
\hline 294.79 & 298.58 & 303.75 & 304.36 & 325.84 & 337.64 & 3154.65 & 3156.84 & 3158.24 & 3161.20 & 3163.54 & 3165.63 \\
\hline
\end{tabular}




\begin{tabular}{|c|c|c|c|c|c|}
\hline 3167.65 & 3168.51 & 3172.41 & 3172.90 & 3173.04 & 3174.95 \\
\hline 3184.33 & 3201.41 & 3203.21 & 3219.08 & 3253.48 & 3270.91 \\
\hline$===$ & $==$ & $===$ & $:=$ & & $:=$ \\
\hline & & & & & \\
\hline$====$ & $====$ & $====$ & $=====$ & $===$ & $====$ \\
\hline 84.88 & 85.11 & 116.33 & 117.51 & 136.38 & 155.67 \\
\hline 198.14 & 199.03 & 214.64 & 221.05 & 222.33 & 239.93 \\
\hline 261.09 & 262.60 & 273.08 & 278.46 & 279.01 & 294.16 \\
\hline 300.98 & 301.39 & 324.05 & 349.75 & 362.71 & 365.57 \\
\hline 385.86 & 397.70 & 398.22 & 432.74 & 432.90 & 437.65 \\
\hline 484.14 & 485.04 & 523.11 & 574.85 & 590.41 & 590.56 \\
\hline 816.10 & 816.23 & 817.48 & 939.36 & 941.23 & 941.63 \\
\hline 950.38 & 951.92 & 952.16 & 976.39 & 976.60 & 981.68 \\
\hline 1038.61 & 1045.28 & 1045.41 & 1054.06 & 1054.15 & 1054.28 \\
\hline 1203.95 & 1204.05 & 1210.10 & 1213.58 & 1220.79 & 1220.92 \\
\hline 1233.81 & 1234.02 & 1236.09 & 1407.99 & 1410.88 & 1411.04 \\
\hline 1416.85 & 1416.95 & 1419.52 & 1443.28 & 1443.33 & 1454.36 \\
\hline 1487.05 & 1487.24 & 1489.85 & 1498.43 & 1501.71 & 1501.82 \\
\hline 1502.06 & 1510.98 & 1511.26 & 1518.26 & 1518.37 & 1518.62 \\
\hline 1529.73 & 1530.12 & 1533.88 & 1543.76 & 1543.85 & 1544.96 \\
\hline 3029.34 & 3029.57 & 3029.76 & 3033.61 & 3033.90 & 3034.04 \\
\hline 3039.16 & 3039.29 & 3042.64 & 3099.41 & 3100.79 & 3100.90 \\
\hline 3105.5 & 3105.73 & 3106.18 & 3114.89 & 3115.11 & 3120.05 \\
\hline 3144.90 & 3145.54 & 3146.66 & 3155.63 & 3159.73 & 3160.20 \\
\hline 77 & 178.24 & & & & \\
\hline
\end{tabular}

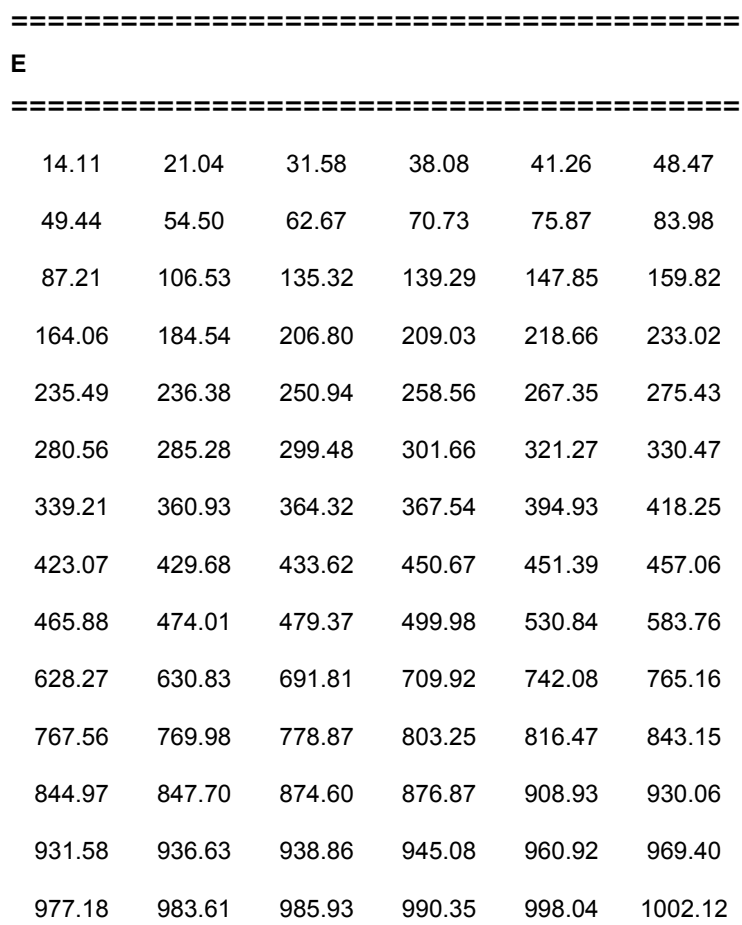

\begin{tabular}{|c|c|c|c|c|c|}
\hline 1025.18 & 048.24 & 1057.80 & 1062.29 & 1064.03 & 1066.40 \\
\hline 1072.98 & 1087.83 & 1092.91 & 1116.32 & 1148.06 & 1156.60 \\
\hline 1165.94 & 82.98 & 193.27 & 195.31 & 210.46 & 1216.88 \\
\hline 1226.29 & 230.66 & 272.32 & 279.24 & 281.62 & 286.62 \\
\hline 1298.85 & 308.17 & 310.68 & 344.10 & 346.45 & 365.25 \\
\hline 1378.90 & 94.88 & 407.12 & 408.38 & 1409.03 & 410.93 \\
\hline 1417.17 & 433.25 & 1441.08 & 462.50 & 465.94 & 476.71 \\
\hline 1481.19 & 483.86 & 488.47 & 1493.60 & 1495.79 & 501.30 \\
\hline 1504.40 & 504.68 & 505.11 & 507.55 & 1509.77 & 1509.96 \\
\hline 1512.56 & 518.26 & 520.95 & 522.57 & 53 & 524.45 \\
\hline 1536.6 & 546. & 1548 & 630 & 8 & 756 \\
\hline 1768.9 & 395. & 2998.80 & 007 & 77 & 038.23 \\
\hline 3042.8 & 044.08 & 3048.32 & 049 & 23 & 3083.40 \\
\hline 3102.7 & 07 & 110.26 & 111 & 14.11 & 114. \\
\hline 3116.4 & 22 & 123 & 133 & 5 & 161. \\
\hline 3162.30 & 65. & 67 & 169 & 8 & 172. \\
\hline 3178.92 & 198.49 & 3234.89 & 3256.02 & 3539.12 & 3557. \\
\hline \multirow{2}{*}{\multicolumn{6}{|c|}{ 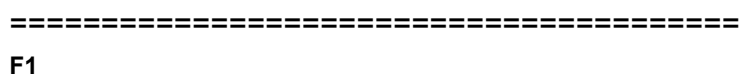 }} \\
\hline \multirow{2}{*}{\multicolumn{6}{|c|}{ 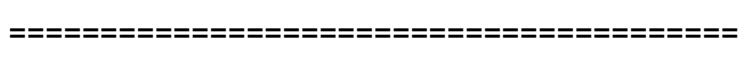 }} \\
\hline & & & & & \\
\hline 22.43 & 261 & 31.68 & 36 & 40.25 & 41. \\
\hline 49.17 & 54.0 & 54 & 58. & 61 & 66. \\
\hline 74.16 & 1.4 & 83.66 & 92.8 & 95.98 & 101.56 \\
\hline 102.2 & 112. & 15 & 119 & 124 & 144 \\
\hline 148.0 & 158. & 66. & 171 & 176 & \\
\hline 185. & 15 & 209. & 211. & 224 & 234.57 \\
\hline 6 & & & & & \\
\hline 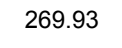 & & & & & \\
\hline & & & & & \\
\hline 347.2 & 363.1 & 365 & 371.5 & & \\
\hline 421.7 & 432 & 3 & 436 & & \\
\hline 465.4 & 466 & 4 & 475 & & \\
\hline . & 528.8 & 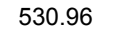 & & & 001.20 \\
\hline 631.6 & & & & & \\
\hline 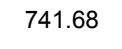 & 750. & & & & \\
\hline 785.14 & 802. & 年 & . & 84.01 & 列 \\
\hline 850.37 & & & & & \\
\hline 917.35 & & & & & \\
\hline 9 & 9 & 973.72 & 977. & & \\
\hline 986. & & & 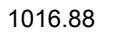 & & \\
\hline 59 & & $100<$ & 8 & 1067 & 107 \\
\hline $10 / 6.10$ & $.5<$ & 98.81 & 05.25 & 115.01 & 12 \\
\hline
\end{tabular}




\begin{tabular}{|c|c|c|c|c|c|}
\hline 1153.11 & 1168.48 & 1174.25 & 1178.05 & 1184.72 & 1189.77 \\
\hline 1195.13 & 1207.99 & 1215.30 & 1217.75 & 1224.40 & 1235.23 \\
\hline 1237.20 & 1247.57 & 1252.23 & 1272.46 & 1279.60 & 1280.12 \\
\hline 1280.55 & 1284.81 & 1287.23 & 1296.62 & 1313.32 & 1315.41 \\
\hline 1319.17 & 1329.19 & 1343.38 & 1344.77 & 1359.02 & 1363.27 \\
\hline 1364.85 & 1372.59 & 1399.02 & 1407.98 & 1411.66 & 1412.46 \\
\hline 1418.73 & 1424.65 & 1435.87 & 1442.77 & 1466.15 & 1476.96 \\
\hline 1482.28 & 1484.30 & 1486.21 & 1489.70 & 1496.29 & 1497.86 \\
\hline 1500.30 & 1501.91 & 1503.79 & 1504.27 & 1504.88 & 1507.07 \\
\hline 1509.19 & 1509.96 & 1515.40 & 1519.70 & 1522.13 & 1522.83 \\
\hline 1524.88 & 1525.81 & 1527.94 & 1538.13 & 1539.29 & 1545.92 \\
\hline 1549.68 & 1582.94 & 1632.08 & 1655.73 & 1750.49 & 1772.87 \\
\hline 2984.35 & 2990.63 & 3004.32 & 3007.17 & 3043.10 & 3043.96 \\
\hline 3044.26 & 3051.08 & 3051.45 & 3052.91 & 3054.68 & 3058.17 \\
\hline 3080.06 & 3096.17 & 3097.98 & 3109.18 & 3109.67 & 3112.10 \\
\hline 3115.10 & 3119.76 & 3120.29 & 3125.70 & 3127.73 & 3128.41 \\
\hline 3135.67 & 3137.30 & 3141.17 & 3143.28 & 3161.64 & 3165.09 \\
\hline 3167.37 & 3169.16 & 3172.08 & 3173.38 & 3181.79 & 3183.59 \\
\hline 3201.91 & 3241.47 & 3246.34 & 3248.13 & 3511.54 & 3561.94 \\
\hline
\end{tabular}

\begin{tabular}{|c|c|c|c|c|c|}
\hline \multicolumn{6}{|l|}{ F1-TS } \\
\hline \multicolumn{6}{|c|}{ 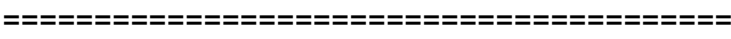 } \\
\hline-323.23 & 10.94 & 20.02 & 26.67 & 34.49 & 39.04 \\
\hline 43.59 & 46.75 & 48.05 & 51.10 & 56.56 & 61.55 \\
\hline 68.22 & 68.87 & 78.26 & 90.82 & 91.56 & 93.23 \\
\hline 98.42 & 108.40 & 115.74 & 120.11 & 122.80 & 131.32 \\
\hline 144.68 & 149.93 & 154.35 & 156.13 & 179.30 & 187.23 \\
\hline 189.73 & 192.84 & 197.73 & 202.57 & 206.32 & 215.74 \\
\hline 222.44 & 232.43 & 236.92 & 237.91 & 242.80 & 249.47 \\
\hline 257.31 & 263.05 & 266.97 & 272.56 & 280.35 & 286.85 \\
\hline 292.52 & 301.71 & 308.38 & 314.01 & 323.59 & 326.76 \\
\hline 339.88 & 355.30 & 357.17 & 367.47 & 370.56 & 394.26 \\
\hline 405.55 & 422.99 & 425.00 & 434.29 & 436.23 & 448.56 \\
\hline 457.26 & 462.15 & 467.28 & 473.35 & 479.76 & 493.22 \\
\hline 498.50 & 508.36 & 531.01 & 553.33 & 558.53 & 581.47 \\
\hline 598.88 & 612.04 & 629.77 & 635.91 & 687.64 & 695.01 \\
\hline 711.70 & 741.11 & 757.08 & 764.77 & 772.35 & 775.90 \\
\hline 782.00 & 790.87 & 805.92 & 818.59 & 823.78 & 841.60 \\
\hline 844.41 & 847.96 & 853.06 & 873.25 & 891.23 & 907.00 \\
\hline 909.76 & 916.60 & 929.85 & 933.80 & 937.95 & 939.63 \\
\hline 948.09 & 959.91 & 968.79 & 971.16 & 977.42 & 986.56 \\
\hline 987.27 & 994.87 & 996.26 & 1012.20 & 1017.44 & 1021.87 \\
\hline
\end{tabular}

\begin{tabular}{|c|c|c|c|c|c|}
\hline 1025.16 & 1056.54 & 1058.99 & 1060.04 & 1061.77 & 1064.93 \\
\hline 1066.98 & 1072.53 & 1082.80 & 1103.74 & 1116.17 & 1116.79 \\
\hline 1149.55 & 1150.25 & 1161.55 & 1174.82 & 1187.74 & 1191.89 \\
\hline 1196.01 & 1196.30 & 1201.07 & 1207.42 & 1217.92 & 1221.43 \\
\hline 1226.00 & 1231.37 & 1239.85 & 1263.17 & 1264.47 & 1272.53 \\
\hline 1279.72 & 1281.59 & 1287.54 & 1298.78 & 1305.40 & 1312.97 \\
\hline 1316.16 & 1318.70 & 1339.68 & 1340.69 & 1343.97 & 1352.82 \\
\hline 1366.26 & 1372.90 & 1400.20 & 1408.12 & 1412.79 & 1413.34 \\
\hline 1419.59 & 1422.31 & 1437.48 & 1443.46 & 1465.21 & 1468.13 \\
\hline 1480.75 & 1483.45 & 1484.15 & 1485.48 & 1492.23 & 1494.37 \\
\hline 1497.74 & 1497.88 & 1500.46 & 1504.03 & 1505.24 & 1505.51 \\
\hline 1507.48 & 1509.42 & 1512.85 & 1514.61 & 1516.88 & 1522.08 \\
\hline 1523.32 & 1523.67 & 1526.60 & 1534.75 & 1542.78 & 1544.60 \\
\hline 1548.65 & 1632.44 & 1640.88 & 1657.06 & 1739.77 & 1776.64 \\
\hline 2985.67 & 2994.09 & 2995.86 & 3011.91 & 3013.95 & 3045.46 \\
\hline 3045.91 & 3048.04 & 3051.85 & 3055.77 & 3058.15 & 3061.63 \\
\hline 3078.56 & 3092.41 & 3109.98 & 3110.96 & 3111.74 & 3114.41 \\
\hline 3121.63 & 3126.95 & 3129.16 & 3130.05 & 3132.79 & 3132.95 \\
\hline 3138.06 & 3140.45 & 3142.14 & 3152.33 & 3153.16 & 3163.62 \\
\hline 3168.69 & 3171.27 & 3172.57 & 3175.76 & 3184.65 & 3187.29 \\
\hline 3201.28 & 3214.74 & 3231.16 & 3279.01 & 3548.12 & 3561.04 \\
\hline
\end{tabular}

\begin{tabular}{|c|c|c|c|c|c|}
\hline \multicolumn{6}{|l|}{ F2-TS } \\
\hline \multicolumn{6}{|c|}{ 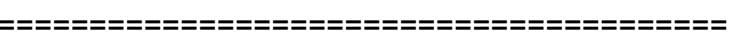 } \\
\hline-295.90 & 12.35 & 26.32 & 29.17 & 34.73 & 39.29 \\
\hline 45.55 & 47.13 & 51.17 & 55.01 & 55.87 & 63.38 \\
\hline 65.11 & 68.15 & 78.01 & 84.15 & 88.54 & 91.41 \\
\hline 94.16 & 98.07 & 109.37 & 116.28 & 119.00 & 124.35 \\
\hline 143.70 & 144.87 & 153.93 & 155.73 & 158.03 & 183.03 \\
\hline 188.08 & 190.48 & 196.86 & 199.68 & 206.80 & 214.43 \\
\hline 226.68 & 229.60 & 237.92 & 238.14 & 245.04 & 251.58 \\
\hline 256.78 & 259.18 & 267.78 & 272.28 & 277.88 & 285.57 \\
\hline 290.61 & 297.89 & 307.12 & 307.55 & 315.19 & 326.81 \\
\hline 330.97 & 340.60 & 350.83 & 358.89 & 364.30 & 370.82 \\
\hline 388.95 & 400.14 & 406.75 & 423.12 & 432.98 & 435.87 \\
\hline 447.22 & 455.86 & 457.69 & 462.90 & 466.58 & 473.06 \\
\hline 479.40 & 487.21 & 493.67 & 498.97 & 507.19 & 533.07 \\
\hline 553.79 & 558.73 & 581.61 & 608.34 & 629.76 & 637.00 \\
\hline 668.14 & 685.89 & 713.59 & 739.67 & 756.88 & 763.52 \\
\hline 770.00 & 779.02 & 784.43 & 793.73 & 806.65 & 817.08 \\
\hline 823.58 & 835.83 & 844.20 & 849.04 & 852.54 & 853.49 \\
\hline 873.58 & 896.04 & 903.45 & 914.23 & 921.75 & 928.77 \\
\hline
\end{tabular}




\begin{tabular}{llllll}
933.17 & 937.93 & 938.17 & 939.56 & 950.59 & 964.97 \\
972.85 & 974.93 & 976.74 & 986.58 & 991.66 & 993.33 \\
1013.31 & 1018.19 & 1022.09 & 1037.52 & 1056.17 & 1059.91 \\
1061.48 & 1063.08 & 1066.66 & 1072.32 & 1081.17 & 1088.42 \\
1095.06 & 1105.00 & 1114.34 & 1117.49 & 1149.57 & 1162.41 \\
1165.24 & 1175.46 & 1179.28 & 1187.62 & 1190.88 & 1195.05 \\
1197.45 & 1206.99 & 1215.98 & 1222.55 & 1230.14 & 1239.58 \\
1263.05 & 1267.92 & 1270.28 & 1275.00 & 1279.74 & 1282.64 \\
1287.88 & 1299.62 & 1310.56 & 1316.57 & 1318.98 & 1334.57 \\
1338.31 & 1341.76 & 1347.28 & 1358.09 & 1365.59 & 1380.26 \\
1383.21 & 1393.17 & 1400.49 & 1408.55 & 1413.13 & 1413.59 \\
1419.66 & 1422.95 & 1438.17 & 1443.55 & 1464.49 & 1468.47 \\
1474.06 & 1480.54 & 1483.96 & 1484.07 & 1484.93 & 1492.48 \\
1495.21 & 1497.51 & 1497.80 & 1499.78 & 1503.99 & 1505.16 \\
1506.02 & 1507.70 & 1509.69 & 1510.08 & 1513.18 & 1516.97 \\
1521.71 & 1522.85 & 1523.75 & 1527.53 & 1534.92 & 1542.00 \\
\hline 1544.00 & 1549.22 & 1639.89 & 1655.98 & 1706.16 & 1736.53 \\
\hline 1773.87 & 2984.49 & 2992.99 & 2993.66 & 3008.17 & 3010.19 \\
3044.82 & 3046.59 & 3047.60 & 3048.08 & 3051.22 & 3054.75 \\
\hline 3058.07 & 3063.22 & 3075.99 & 3078.03 & 3098.46 & 3107.49 \\
3110.30 & 3111.56 & 3116.04 & 3119.85 & 3120.73 & 3125.80 \\
3125.86 & 3126.30 & 3130.40 & 3133.61 & 3136.98 & 3141.47 \\
3142.05 & 3148.96 & 3162.46 & 3163.57 & 3170.62 & 3174.83 \\
3174.88 & 3183.84 & 3187.19 & 3198.19 & 3207.64 & 3226.92 \\
3229.00 & 3551.18 & 3563.97 & & & \\
\hline & & & & & \\
\hline 13
\end{tabular}

\begin{tabular}{|c|c|c|c|c|c|}
\hline-278.74 & 12.31 & 18.66 & 31.92 & 35.55 & 37.85 \\
\hline 41.26 & 44.78 & 48.91 & 56.94 & 60.54 & 61.50 \\
\hline 68.06 & 70.37 & 73.13 & 79.68 & 87.47 & 94.32 \\
\hline 95.96 & 101.56 & 106.97 & 109.41 & 115.04 & 121.87 \\
\hline 131.24 & 139.21 & 144.68 & 146.06 & 153.92 & 175.96 \\
\hline 180.07 & 191.35 & 193.82 & 196.18 & 200.86 & 205.96 \\
\hline 207.18 & 217.40 & 224.65 & 229.70 & 236.49 & 241.01 \\
\hline 251.68 & 258.00 & 265.58 & 268.95 & 271.97 & 279.05 \\
\hline 282.98 & 291.23 & 295.72 & 301.26 & 305.73 & 313.56 \\
\hline 324.81 & 330.31 & 340.26 & 341.23 & 353.30 & 359.65 \\
\hline 368.18 & 371. & 391.09 & 403.64 & 412.73 & 422.50 \\
\hline 424.21 & 430.01 & 435.07 & 447.86 & 459.20 & 464.77 \\
\hline 467.59 & 473.63 & 480.15 & 488.41 & 497.70 & 506.74 \\
\hline 532.5 & 553.35 & 558.59 & 561.65 & 579.06 & 603.60 \\
\hline
\end{tabular}

\begin{tabular}{|c|c|c|c|c|c|}
\hline 631.24 & 636.07 & 638.07 & 686.54 & 712.24 & 739.71 \\
\hline 757.22 & 764.35 & 768.80 & 771.47 & 776.34 & 782.63 \\
\hline 788.17 & 804.74 & 824.12 & 826.41 & 843.38 & 347.99 \\
\hline 850.16 & 854.65 & 871.67 & 872.96 & 882.09 & 900.02 \\
\hline 916.74 & 918.93 & 929.76 & 932.30 & 937.18 & 939.46 \\
\hline 948.07 & 963.96 & 966.01 & 970.01 & 977.49 & 986.31 \\
\hline 989.24 & 995.06 & 998.36 & 1011.84 & 1017.78 & 1019.84 \\
\hline 1021.70 & 1055.35 & 1056.03 & 1057.05 & 1061.88 & 1062.83 \\
\hline 1066.36 & 1071.19 & 1079.42 & 1098.23 & 1103.95 & 1114.14 \\
\hline 1115.86 & 1124.46 & 1154.10 & 1165.94 & 1174.57 & 1174.90 \\
\hline 1184.82 & 1188.41 & 1194.62 & 1200.25 & 1206.23 & 1215.90 \\
\hline 1219.28 & 1221.78 & 1230.36 & 1237.68 & 1240.54 & 1262.53 \\
\hline 1271.90 & 275.94 & 1279.02 & 1281.24 & 1286.86 & 1298.51 \\
\hline 1303.61 & 314.83 & 1319.06 & 1324.98 & 1333.74 & 1336.15 \\
\hline 1344.05 & 1350.91 & 1365.13 & 1366.11 & 1380.81 & 1381.86 \\
\hline 1399.82 & 1400.19 & 1406.33 & 1408.57 & 1412.59 & 1413.02 \\
\hline 1419.43 & 1425.72 & 1437.32 & 1443.16 & 1465.63 & 1474.70 \\
\hline 1481.36 & 1483.98 & 1484.82 & 1488.02 & 1488.62 & 1495.04 \\
\hline 1496.95 & 1498.66 & 1499.41 & 1503.19 & 1503.98 & 1505.08 \\
\hline 1505.73 & 508.20 & 1509.29 & 1513.50 & 1514.14 & 1515.69 \\
\hline 1518.74 & 522.31 & 1523.91 & 1525.28 & 1528.27 & 1536.35 \\
\hline 41 & 545 & 54 & 04 & 37 & 1707.92 \\
\hline 1741.16 & 1771.38 & 2985.53 & 2990.66 & 2995.03 & 3000.31 \\
\hline 3030.46 & 3043.95 & 3044.26 & 3046.33 & 3046.81 & 3048.99 \\
\hline 3051.89 & 3053.16 & 3057.09 & 3058.12 & 3061.76 & 3069.99 \\
\hline 3087.66 & 3093.77 & 3101.63 & $310 \varepsilon$ & 3109.04 & 3110.71 \\
\hline 3112.64 & 114.43 & 3114.65 & 3124 & 3126.23 & 3132.11 \\
\hline 3133. & 3137.60 & 3145.18 & 3147 & 3162.24 & 3169.68 \\
\hline 3172. & 3173.68 & 3174.79 & 3175.68 & 3176.82 & 3183.89 \\
\hline 3198.6 & 3206.58 & 3215.53 & 3227.87 & 3535.12 & 3578.42 \\
\hline \multicolumn{6}{|c|}{ 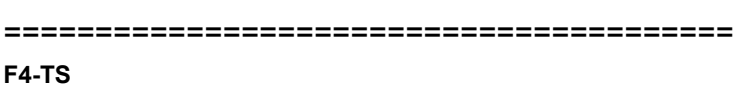 } \\
\hline \multicolumn{6}{|c|}{ 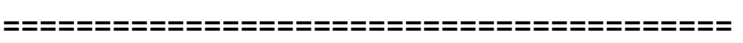 } \\
\hline-254.6 & 14.9 & 18.81 & 29.76 & 34.85 & 40.13 \\
\hline 4 & 48. & 48. & 52 & 56. & 58 \\
\hline 1.72 & 68 & 70.4 & 74. & 81.14 & 89.01 \\
\hline 90.00 & 94.32 & 98.5 & 110. & 116.85 & 120.45 \\
\hline 124.9 & 142.8 & 145 & 153. & 156.49 & 158.50 \\
\hline 183.3 & 185.42 & 192.2 & 198. & 201.86 & 207.39 \\
\hline 209 & 2 & 226.79 & 234. & 238.86 & 245.55 \\
\hline & 256. & 260 & 260 & 269 & \\
\hline 276.73 & 20 & 21 & 291.19 & 300.66 & 311.04 \\
\hline
\end{tabular}




\begin{tabular}{|c|c|c|c|c|c|}
\hline 315.70 & 318.96 & 327.94 & 338.93 & 350.51 & 352.26 \\
\hline 359.70 & 365.88 & 369.11 & 388.74 & 392.99 & 405.68 \\
\hline 419.98 & 434.10 & 435.10 & 437.57 & 445.87 & 458.01 \\
\hline 463.16 & 468.07 & 473.00 & 478.98 & 492.96 & 494.30 \\
\hline 503.19 & 512.22 & 525.24 & 531.15 & 552.03 & 557.86 \\
\hline 565.82 & 581.49 & 611.60 & 629.18 & 637.36 & 685.35 \\
\hline 709.87 & 732.06 & 738.42 & 756.55 & 763.69 & 769.53 \\
\hline 770.26 & 777.21 & 782.05 & 805.97 & 817.81 & 827.83 \\
\hline 831.91 & 835.84 & 843.19 & 847.65 & 849.03 & 871.00 \\
\hline 873.02 & 880.71 & 904.80 & 910.75 & 915.84 & 928.90 \\
\hline 932.64 & 938.08 & 939.03 & 951.21 & 965.50 & 967.10 \\
\hline 976.41 & 978.99 & 984.10 & 986.70 & 990.09 & 999.75 \\
\hline 1011.97 & 1015.92 & 1017.09 & 1023.33 & 1032.56 & 1056.33 \\
\hline 1060.65 & 1061.36 & 1062.94 & 1066.70 & 1072.27 & 1076.39 \\
\hline 1080.03 & 1102.59 & 1105.86 & 1111.81 & 1115.18 & 1125.71 \\
\hline 1146.36 & 1161.73 & 1169.72 & 1173.24 & 1187.53 & 1190.63 \\
\hline 1193.21 & 1194.11 & 1204.96 & 1206.60 & 1215.88 & 1223.05 \\
\hline 1229.26 & 1237.82 & 1242.15 & 1263.44 & 1270.34 & 1276.45 \\
\hline 1280.18 & 1281.51 & 1287.87 & 1289.47 & 1298.68 & 1313.19 \\
\hline 1315.36 & 1318.21 & 1319.47 & 1329.85 & 1337.72 & 1343.53 \\
\hline 1358.39 & 1365.84 & 1375.18 & 1376.69 & 1381.97 & 1388.03 \\
\hline 1399.07 & 1400.24 & 1408.40 & 1413.02 & 1414.55 & 1414.72 \\
\hline 1419.51 & 1421.86 & 1440.46 & 1443.57 & 1456.24 & 1463.84 \\
\hline 1468.01 & 1481.42 & 1483.87 & 1484.77 & 1492.31 & 1494.63 \\
\hline 1495.37 & 1497.46 & 1498.01 & 1498.68 & 1501.83 & 1504.00 \\
\hline 1504.78 & 1506.11 & 1507.88 & 1510.28 & 1514.27 & 1516.76 \\
\hline 1520.22 & 1521.58 & 1522.26 & 1523.30 & 1523.89 & 1529.14 \\
\hline 1533.09 & 1540.83 & 1543.07 & 1549.11 & 1638.38 & 1655.03 \\
\hline 1709.82 & 1740.33 & 1775.47 & 2985.81 & 2992.31 & 2997.04 \\
\hline 3009.61 & 3018.87 & 3022.26 & 3035.18 & 3044.56 & 3045.06 \\
\hline 3045.67 & 3047.96 & 3050.01 & 3053.71 & 3055.21 & 3057.35 \\
\hline 3059.04 & 3066.69 & 3069.73 & 3091.12 & 3104.15 & 3105.20 \\
\hline 3110.35 & 3110.70 & 3111.06 & 3112.50 & 3114.70 & 3122.05 \\
\hline 3125.45 & 3128.60 & 3129.59 & 3131.66 & 3132.30 & 3138.50 \\
\hline 3142.27 & 3151.33 & 3160.75 & 3166.58 & 3169.45 & 3169.52 \\
\hline 3173.27 & 3182.88 & 3193.89 & 3199.93 & 3213.03 & 3224.81 \\
\hline 234.89 & 3548.02 & 35 & & & \\
\hline
\end{tabular}

\section{F5}

\begin{tabular}{|c|c|c|c|c|c|}
\hline 14.36 & 22.81 & 25.08 & 33.49 & 35.10 & 40.05 \\
\hline 43.50 & 48.60 & 53.70 & 55.63 & 57.92 & 63.41 \\
\hline
\end{tabular}

\begin{tabular}{|c|c|c|c|c|c|}
\hline 65.47 & 70.11 & 74.16 & 84.02 & 86.06 & 90.38 \\
\hline 95.43 & 97.37 & 102.58 & 105.91 & 113.82 & 116.40 \\
\hline 124.57 & 145.06 & 150.73 & 159.89 & 171.10 & 174.27 \\
\hline 183.01 & 184.78 & 187.42 & 194.54 & 197.20 & 206.69 \\
\hline 211.21 & 221.57 & 230.65 & 233.34 & 235.67 & 237.95 \\
\hline 245.88 & 257.37 & 261.89 & 262.77 & 267.58 & 275.34 \\
\hline 277.27 & 283.45 & 292.76 & 293.59 & 304.90 & 308.43 \\
\hline 311.98 & 316.03 & 326.95 & 339.68 & 348.88 & 356.28 \\
\hline 366.01 & 368.01 & 369.12 & 387.06 & 394.10 & 403.56 \\
\hline 412.44 & 424.96 & 434.02 & 439.79 & 441.33 & 447.93 \\
\hline 457.54 & 468.67 & 470.50 & 471.56 & 475.99 & 480.20 \\
\hline 496.67 & 500.42 & 502.19 & 530.79 & 533.35 & 547.49 \\
\hline 552.07 & 581.91 & 597.98 & 628.82 & 634.25 & 637.72 \\
\hline 686.16 & 690.85 & 711.97 & 724.34 & 739.14 & 761.79 \\
\hline 765.69 & 768.85 & 775.45 & 780.49 & 787.36 & 800.18 \\
\hline 803.30 & 819.53 & 831.68 & 835.48 & 841.46 & 845.58 \\
\hline 852.46 & 875.11 & 884.13 & 902.78 & 913.00 & 921.75 \\
\hline 929.80 & 931.47 & 935.52 & 937.56 & 939.28 & 952.49 \\
\hline 955.90 & 970.68 & 977.11 & 977.95 & 980.73 & 984.67 \\
\hline 986.85 & 1000.38 & 1007.73 & 1010.67 & 1012.99 & 1021.13 \\
\hline 1038.90 & 1045.30 & 1056.66 & 1057.71 & 1061.81 & 1063.65 \\
\hline 1066.78 & 1072.00 & 1081.50 & 1081.95 & 1090.01 & 1098.47 \\
\hline 1115.23 & 1116.43 & 1127.16 & 1130.76 & 1151.03 & 1167.81 \\
\hline 1172.95 & 1182.37 & 1188.06 & 1193.92 & 1198.11 & 1209.46 \\
\hline 1212.09 & 1215.50 & 1224.33 & 1225.99 & 1235.04 & 1235.93 \\
\hline 1250.52 & 1261.53 & 1271.45 & 1274.81 & 1279.95 & 1280.00 \\
\hline 1286.41 & 1295.40 & 1297.95 & 1313.23 & 1313.46 & 1321.83 \\
\hline 1334.12 & 1340.23 & 1342.90 & 1345.88 & 1354.79 & 1364.79 \\
\hline 1366.73 & 1372.87 & 1380.76 & 1388.20 & 1393.45 & 1399.64 \\
\hline 1405.58 & 1407.51 & 1411.88 & 1412.46 & 1412.81 & 1418.98 \\
\hline 1425.22 & 1427.35 & 1437.00 & 1442.87 & 1462.17 & 1472.37 \\
\hline 1481.49 & 1483.53 & 1484.09 & 1484.73 & 1493.35 & 1495.46 \\
\hline 1495.73 & 1499.67 & 1500.16 & 1501.76 & 1503.81 & 1504.14 \\
\hline 1504.60 & 1505.72 & 1507.50 & 1510.15 & 1511.11 & 1513.14 \\
\hline 1514.84 & 1517.70 & 1523.00 & 1523.26 & 1524.18 & 1526.67 \\
\hline 1529.24 & 1533.22 & 1539.15 & 1545.39 & 1548.97 & 1617.09 \\
\hline 1635.91 & 1655.18 & 1743.54 & 1769.91 & 2983.58 & 2985.20 \\
\hline 3003.41 & 3006.33 & 3011.91 & 3030.88 & 3032.01 & 3036.67 \\
\hline 3042.45 & 3043.04 & 3043.30 & 3046.68 & 3049.01 & 3049.81 \\
\hline 3050.60 & 3056.95 & 3070.47 & 3073.76 & 3074.81 & 3093.11 \\
\hline 3097.15 & 3106.89 & 3107.47 & 3108.44 & 3108.52 & 3110.55 \\
\hline 3114.93 & 3120.92 & 3123.42 & 3126.22 & 3130.12 & 3133.00 \\
\hline
\end{tabular}


$\begin{array}{llllll}3139.53 & 3139.73 & 3147.49 & 3151.59 & 3158.12 & 3161.11 \\ 3168.80 & 3168.93 & 3172.25 & 3173.52 & 3176.29 & 3177.46 \\ 3181.91 & 3200.45 & 3226.64 & 3235.03 & 3552.12 & 3556.71\end{array}$

\section{ニニニニ
F5-TS}

\begin{tabular}{|c|c|c|c|c|c|}
\hline-238.82 & 15.18 & 20.87 & 22.08 & 34.99 & 39.31 \\
\hline 46.49 & 48.35 & 49.57 & 55.71 & 59.85 & 62.40 \\
\hline 65.85 & 66.15 & 69.48 & 76.78 & 82.75 & 85.24 \\
\hline 89.55 & 95.70 & 98.61 & 110.74 & 112.42 & 115.26 \\
\hline 121.99 & 129.64 & 143.08 & 152.99 & 155.94 & 162.52 \\
\hline 173.62 & 186.88 & 187.04 & 189.85 & 196.89 & 202.19 \\
\hline 205.59 & 207.74 & 213.49 & 215.32 & 226.57 & 231.73 \\
\hline 238.84 & 244.00 & 250.20 & 257.77 & 259.52 & 267.88 \\
\hline 273.14 & 280.11 & 284.80 & 287.39 & 293.02 & 306.00 \\
\hline 309.56 & 316.93 & 319.31 & 331.70 & 339.33 & 349.31 \\
\hline 350.33 & 353.55 & 362.67 & 369.88 & 377.56 & 389.31 \\
\hline 399.33 & 405.68 & 420.78 & 435.09 & 435.64 & 446.56 \\
\hline 457.59 & 459.97 & 466.29 & 467.42 & 473.14 & 479.53 \\
\hline 489.43 & 494.12 & 497.43 & 503.81 & 531.43 & 532.44 \\
\hline 554.44 & 560.11 & 571.41 & 582.65 & 612.31 & 628.86 \\
\hline 637.45 & 685.36 & 711.93 & 718.52 & 738.32 & 752.43 \\
\hline 760.88 & 763.38 & 769.82 & 777.91 & 783.29 & 796.23 \\
\hline 803.77 & 806.57 & 813.42 & 823.36 & 843.50 & 848.55 \\
\hline 848.94 & 851.47 & 866.57 & 873.00 & 903.43 & 917.89 \\
\hline 920.13 & 928.75 & 933.35 & 935.33 & 938.05 & 939.24 \\
\hline 951.95 & 961.60 & 966.82 & 970.46 & 976.27 & 978.84 \\
\hline 986.78 & 987.35 & 991.83 & 1014.34 & 1017.26 & 1023.17 \\
\hline 1026.59 & 1037.04 & 1056.20 & 1060.76 & 1061.32 & 1062.71 \\
\hline 1066.63 & 1068.86 & 1072.66 & 1080.77 & 1082.10 & 1103.91 \\
\hline 1108.32 & 1114.78 & 1117.77 & 1124.11 & 1149.37 & 1163.04 \\
\hline 1174.51 & 1179.40 & 1188.53 & 1191.55 & 1194.28 & 1195.73 \\
\hline 1207.19 & 1209.32 & 1216.31 & 1221.54 & 1223.54 & 1228.60 \\
\hline 1238.85 & 1256.43 & 1261.68 & 1269.81 & 1279.95 & 1281.55 \\
\hline 1283.40 & 1288.30 & 1298.18 & 1314.51 & 1317.71 & 1318.59 \\
\hline 1324.49 & 1328.31 & 1339.47 & 1340.81 & 1342.56 & 1357.54 \\
\hline 1364.90 & 1366.23 & 1369.84 & 1381.93 & 1391.36 & 1400.40 \\
\hline 1400.83 & 1409.00 & 1411.62 & 1413.27 & 1414.31 & 1419.58 \\
\hline 1423.13 & 1428.37 & 1439.11 & 1443.60 & 1452.15 & 1467.03 \\
\hline 1468.70 & 1480.84 & 1484.34 & 1485.22 & 1492.61 & 1493.80 \\
\hline 1494.32 & 1498.04 & 1498.38 & 1500.75 & 1501.88 & 1503.31 \\
\hline 1504.10 & 1504.68 & 1506.30 & 1507.74 & 1510.39 & 1514.04 \\
\hline
\end{tabular}

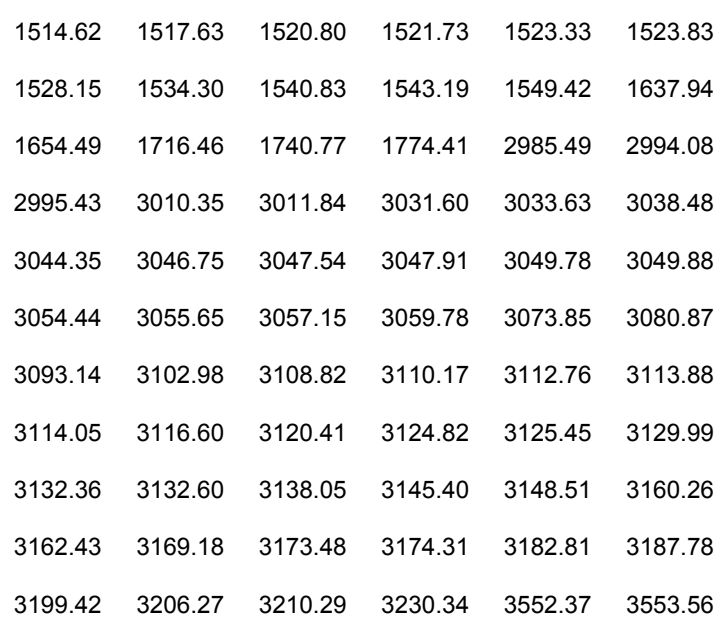




$\begin{array}{llllll}1422.20 & 1427.80 & 1443.41 & 1450.85 & 1460.11 & 1469.03 \\ 1479.91 & 1482.52 & 1483.92 & 1484.88 & 1493.67 & 1496.27 \\ 1499.57 & 1500.47 & 1504.43 & 1505.11 & 1507.28 & 1507.71 \\ 1510.65 & 1511.52 & 1512.62 & 1517.42 & 1517.94 & 1521.14 \\ 1523.49 & 1524.95 & 1528.10 & 1538.28 & 1541.86 & 1547.49 \\ 1562.35 & 1639.41 & 1653.14 & 1656.88 & 1775.19 & 1785.65 \\ 3021.26 & 3023.11 & 3023.50 & 3023.86 & 3035.42 & 3050.18 \\ 3052.30 & 3055.31 & 3057.77 & 3061.55 & 3063.66 & 3067.93 \\ 3074.66 & 3083.38 & 3091.90 & 3102.51 & 3117.77 & 3118.33 \\ 3126.01 & 3130.13 & 3130.68 & 3137.18 & 3139.72 & 3141.45 \\ 3147.74 & 3158.49 & 3158.82 & 3159.66 & 3161.85 & 3170.19 \\ 3171.08 & 3175.52 & 3179.87 & 3183.31 & 3187.95 & 3189.97 \\ 3198.05 & 3208.19 & 3225.84 & 3230.02 & 3303.21 & 3531.35\end{array}$

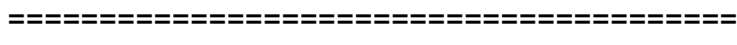
G1-TS

\begin{tabular}{|c|c|c|c|c|c|}
\hline-148.03 & 15.26 & 26.33 & 34.00 & 35.51 & 43.76 \\
\hline 47.63 & 48.17 & 55.82 & 59.32 & 61.76 & 71.16 \\
\hline 74.73 & 82.74 & 83.94 & 86.68 & 92.54 & 96.23 \\
\hline 100.59 & 103.75 & 117.40 & 126.50 & 133.03 & 136.54 \\
\hline 144.41 & 148.46 & 162.06 & 170.63 & 181.93 & 188.36 \\
\hline 197.85 & 204.56 & 212.43 & 218.59 & 225.05 & 234.15 \\
\hline 235.86 & 238.78 & 241.21 & 247.58 & 252.23 & 261.94 \\
\hline 264.40 & 274.50 & 277.75 & 284.18 & 284.66 & 300.76 \\
\hline 310.02 & 312.03 & 319.25 & 322.56 & 337.05 & 341.12 \\
\hline 342.96 & 345.62 & 359.45 & 362.50 & 369.59 & 383.42 \\
\hline 421.92 & 425.29 & 434.73 & 443.75 & 449.28 & 452.01 \\
\hline 465.75 & 466.54 & 471.87 & 476.62 & 479.38 & 495.44 \\
\hline 503.88 & 505.07 & 528.65 & 555.67 & 561.54 & 598.74 \\
\hline 610.47 & 619.65 & 631.09 & 637.89 & 686.44 & 717.04 \\
\hline 734.94 & 747.87 & 752.61 & 762.21 & 766.85 & 772.20 \\
\hline 787.33 & 799.56 & 804.41 & 833.40 & 838.16 & 847.72 \\
\hline 857.03 & 859.09 & 867.91 & 889.79 & 914.90 & 925.20 \\
\hline 931.29 & 933.52 & 937.31 & 940.70 & 942.35 & 951.03 \\
\hline 953.83 & 976.60 & 982.73 & 986.46 & 988.13 & 992.69 \\
\hline 1000.38 & 1002.55 & 1011.39 & 1013.30 & 1018.28 & 1024.96 \\
\hline 1051.95 & 1053.86 & 1063.15 & 1064.73 & 1067.42 & 1068.16 \\
\hline 1072.69 & 1087.23 & 1093.01 & 1096.50 & 1110.76 & 1147.73 \\
\hline 1148.90 & 1165.34 & 1177.33 & 1178.96 & 1181.38 & 1190.36 \\
\hline 1193.47 & 1196.11 & 1199.75 & 1204.23 & 1212.12 & 1224.27 \\
\hline 1234.17 & 1242.53 & 1243.09 & 1251.01 & 1251.76 & 1267.68 \\
\hline 1275.44 & 1279.27 & 1281.51 & 1290.48 & 1304.03 & 1306.95 \\
\hline
\end{tabular}

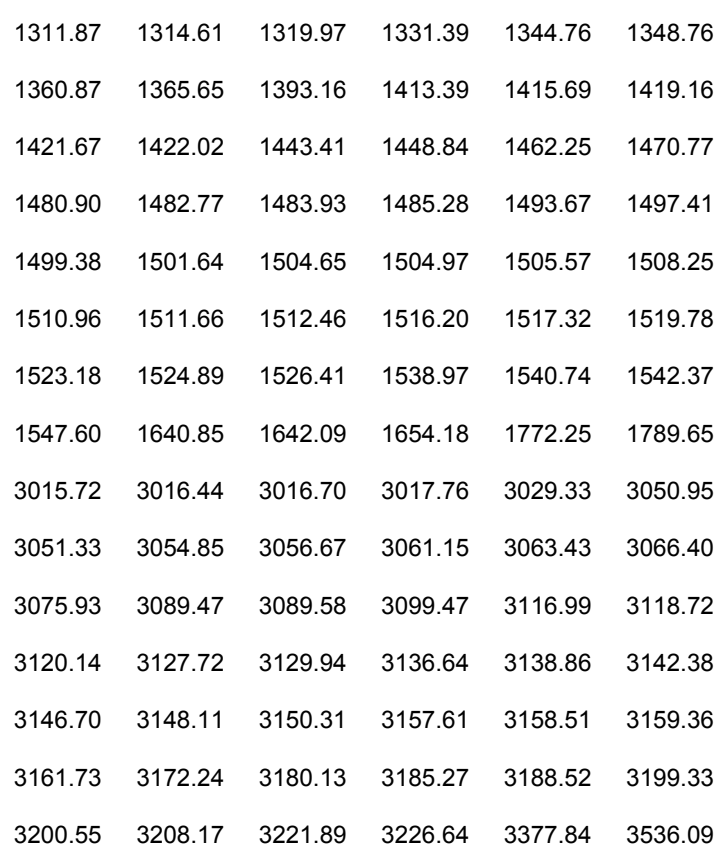

\begin{tabular}{|c|c|c|c|c|c|}
\hline 15.34 & 29.70 & 34.94 & 35.89 & 42.73 & 43.46 \\
\hline 50.02 & 52.40 & 57.01 & 62.69 & 64.62 & 66.87 \\
\hline 73.35 & 75.85 & 82.77 & 88.25 & 93.25 & 97.04 \\
\hline 102.89 & 105.90 & 110.36 & 114.84 & 120.62 & 134.88 \\
\hline 137.92 & 145.59 & 147.55 & 154.15 & 157.11 & 174.74 \\
\hline 177.69 & 189.33 & 194.31 & 199.80 & 206.60 & 208.95 \\
\hline 210.79 & 222.72 & 230.90 & 235.92 & 239.14 & 245.61 \\
\hline 247.38 & 249.96 & 255.36 & 262.44 & 270.86 & 276.48 \\
\hline 279.29 & 288.78 & 296.67 & 298.67 & 302.08 & 309.58 \\
\hline 311.15 & 321.16 & 335.48 & 341.23 & 343.40 & 346.61 \\
\hline 353.54 & 354.57 & 361.72 & 364.10 & 380.09 & 396.28 \\
\hline 399.98 & 425.49 & 430.00 & 436.42 & 448.62 & 458.80 \\
\hline 465.21 & 468.06 & 470.73 & 475.85 & 478.46 & 484.46 \\
\hline 486.68 & 497.82 & 503.44 & 511.22 & 535.01 & 552.86 \\
\hline 556.74 & 561.53 & 599.68 & 612.30 & 617.07 & 627.87 \\
\hline 638.52 & 686.58 & 711.43 & 720.75 & 740.04 & 752.77 \\
\hline 753.86 & 760.82 & 774.85 & 778.64 & 787.33 & 799.13 \\
\hline 807.34 & 808.64 & 820.36 & 829.52 & 844.37 & 850.77 \\
\hline 854.20 & 862.09 & 869.13 & 923.23 & 927.61 & 933.51 \\
\hline 935.74 & 936.53 & 937.81 & 940.08 & 946.13 & 948.78 \\
\hline 969.28 & 974.18 & 981.99 & 982.51 & 984.97 & 987.24 \\
\hline 989.98 & 994.08 & 1007.34 & 1011.80 & 1016.54 & 1023.22 \\
\hline 1038.10 & 1051.61 & 1052.81 & 1059.85 & 1062.87 & 1065.87 \\
\hline 1070.77 & 1078.07 & 1079.74 & 1081.20 & 1091.35 & 1093.64 \\
\hline
\end{tabular}




\begin{tabular}{|c|c|c|c|c|c|}
\hline 1110.73 & 1115.58 & 1126.31 & 1142.91 & 1162.52 & 1172.12 \\
\hline 1173.99 & 1176.13 & 1180.49 & 1192.97 & 1194.37 & 1198.29 \\
\hline 1204.72 & 1211.49 & 1215.12 & 1225.82 & 1227.27 & 1231.09 \\
\hline 1246.05 & 1248.02 & 1249.71 & 1270.01 & 1273.48 & 1275.56 \\
\hline 1280.76 & 1287.64 & 1293.37 & 1296.93 & 1307.76 & 1311.12 \\
\hline 1315.83 & 1330.81 & 1335.81 & 1340.47 & 1341.93 & 1347.41 \\
\hline 1358.77 & 1365.80 & 1366.84 & 1376.24 & 1388.81 & 1393.70 \\
\hline 1401.73 & 1413.36 & 1413.49 & 1415.02 & 1415.71 & 1416.69 \\
\hline 1417.53 & 1421.77 & 1440.44 & 1442.93 & 1461.75 & 1468.57 \\
\hline 1470.93 & 1478.90 & 1482.31 & 1483.71 & 1489.75 & 1492.58 \\
\hline 1494.32 & 1494.71 & 1497.53 & 1499.67 & 1500.33 & 1502.12 \\
\hline 1503.17 & 1505.17 & 1507.40 & 1509.91 & 1510.27 & 1511.63 \\
\hline 1512.17 & 1515.02 & 1517.95 & 1520.12 & 1520.59 & 1522.07 \\
\hline 1524.17 & 1531.91 & 1538.84 & 1543.31 & 1548.78 & 1647.64 \\
\hline 1655.84 & 1706.57 & 1758.53 & 1790.49 & 3013.00 & 3015.68 \\
\hline 3017.88 & 3023.62 & 3029.19 & 3033.49 & 3034.16 & 3036.28 \\
\hline 3041.30 & 3043.14 & 3043.86 & 3045.06 & 3045.54 & 3052.26 \\
\hline 3054.82 & 3055.11 & 3058.04 & 3068.67 & 3071.22 & 3074.75 \\
\hline 3076.84 & 3079.92 & 3083.31 & 3084.20 & 3109.42 & 3111.47 \\
\hline 3112.96 & 3116.41 & 3121.26 & 3132.14 & 3133.24 & 3144.14 \\
\hline 3154.04 & 3154.27 & 3163.99 & 3164.63 & 3168.03 & 3172.33 \\
\hline 3172.62 & 3174.17 & 3174.95 & 3175.86 & 3186.94 & 3189.73 \\
\hline 3202.81 & 3203.72 & 3207.59 & 3240.73 & 3387.89 & 3549.91 \\
\hline \multicolumn{6}{|l|}{ G5-TS } \\
\hline \multicolumn{6}{|c|}{ 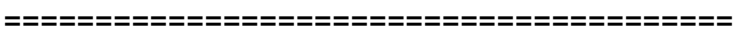 } \\
\hline-153.72 & 20.06 & 26.98 & 36.00 & 40.77 & 41.24 \\
\hline 45.83 & 46.94 & 56.28 & 57.04 & 62.45 & 66.53 \\
\hline 69.19 & 70.21 & 73.83 & 84.00 & 87.21 & 95.56 \\
\hline 105.13 & 107.57 & 112.23 & 118.44 & 129.23 & 136.06 \\
\hline 141.24 & 146.93 & 151.65 & 153.69 & 159.44 & 164.29 \\
\hline 173.89 & 183.64 & 197.47 & 200.50 & 200.93 & 205.69 \\
\hline 212.89 & 218.63 & 223.81 & 224.19 & 228.07 & 232.16 \\
\hline 236.54 & 237.65 & 244.45 & 249.41 & 252.85 & 260.33 \\
\hline 269.52 & 272.64 & 275.40 & 293.29 & 297.86 & 304.07 \\
\hline 307.81 & 312.72 & 332.15 & 335.30 & 336.32 & 346.54 \\
\hline 347.95 & 349.51 & 364.72 & 368.38 & 371.21 & 389.44 \\
\hline 401.98 & 426.03 & 429.09 & 437.31 & 442.63 & 450.18 \\
\hline 463.89 & 466.14 & 469.15 & 470.56 & 472.77 & 481.41 \\
\hline 491.45 & 497.71 & 503.14 & 523.58 & 544.60 & 553.43 \\
\hline 557.11 & 560.32 & 578.89 & 588.30 & 615.82 & 632.28 \\
\hline 639.95 & 688.90 & 716.83 & 721.92 & 748.41 & 751.59 \\
\hline
\end{tabular}

\begin{tabular}{|c|c|c|c|c|c|}
\hline 757.99 & 764.20 & 775.37 & 778.52 & 786.32 & 790.15 \\
\hline 811.40 & 816.61 & 827.92 & 829.33 & 849.50 & 851.78 \\
\hline 858.70 & 860.17 & 873.52 & 923.47 & 924.01 & 934.34 \\
\hline 937.04 & 937.44 & 939.21 & 941.90 & 942.44 & 947.87 \\
\hline 971.80 & 980.07 & 981.90 & 984.72 & 985.14 & 986.77 \\
\hline 990.63 & 999.58 & 1010.18 & 1014.56 & 1020.60 & 1023.37 \\
\hline 1034.05 & 1048.89 & 1051.60 & 1061.20 & 1063.58 & 1066.59 \\
\hline 1070.34 & 1080.38 & 1082.19 & 1083.19 & 1092.51 & 1096.05 \\
\hline 1111.32 & 1112.56 & 1124.35 & 1150.70 & 1161.84 & 1172.06 \\
\hline 1175.36 & 1177.79 & 1181.65 & 1192.97 & 1194.86 & 1199.65 \\
\hline 1202.26 & 1210.02 & 1221.00 & 1222.77 & 1223.71 & 1230.47 \\
\hline 1247.14 & 1248.43 & 1251.17 & 1268.14 & 1272.70 & 1277.17 \\
\hline 1279.87 & 1285.25 & 1292.98 & 1295.96 & 1309.74 & 1312.16 \\
\hline 1314.71 & 1321.35 & 1336.65 & 1338.71 & 1341.00 & 1350.72 \\
\hline 1358.62 & 1361.47 & 1367.63 & 1373.88 & 1392.98 & 1393.17 \\
\hline 1401.91 & 1412.22 & 1414.59 & 1415.33 & 1416.34 & 1417.32 \\
\hline 1418.44 & 1422.36 & 1441.25 & 1442.58 & 1460.84 & 1462.53 \\
\hline 1469.83 & 1482.16 & 1483.10 & 1483.50 & 1493.16 & 1494.08 \\
\hline 1495.03 & 1496.25 & 1498.45 & 1499.50 & 1502.39 & 1503.28 \\
\hline 1503.55 & 1504.46 & 1508.67 & 1508.84 & 1509.62 & 1511.33 \\
\hline 1512.82 & 1515.83 & 1517.92 & 1519.38 & 1520.37 & 1522.92 \\
\hline 1525.32 & 1532.26 & 1539.08 & 1542.35 & 1545.48 & 1645.53 \\
\hline 1656.06 & 1682.22 & 1759.08 & 1806.40 & 3007.99 & 3009.31 \\
\hline 3014.05 & 3018.60 & 3022.03 & 3025.92 & 3027.12 & 3030.56 \\
\hline 3037.20 & 3042.62 & 3045.57 & 3049.14 & 3049.59 & 3050.08 \\
\hline 3053.26 & 3056.34 & 3060.32 & 3064.27 & 3068.38 & 3074.54 \\
\hline 3077.16 & 3088.25 & 3099.77 & 3101.74 & 3103.14 & 3112.28 \\
\hline 3116.53 & 3121.59 & 3127.51 & 3130.66 & 3133.34 & 3133.51 \\
\hline 3138.33 & 3149.44 & 3154.28 & 3165.14 & 3166.91 & 3169.52 \\
\hline 3173.03 & 3173.92 & 3177.21 & 3178.11 & 3193.83 & 3212.12 \\
\hline 3212.62 & 3216.98 & 3220.75 & 3243.85 & 3406.52 & 3548.35 \\
\hline \multicolumn{6}{|c|}{ 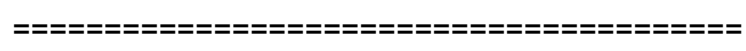 } \\
\hline \multirow{2}{*}{\multicolumn{6}{|c|}{ 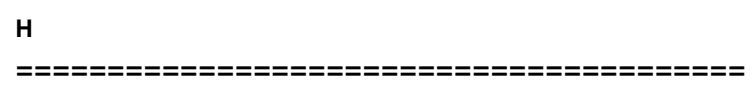 }} \\
\hline & & & & & \\
\hline 24.77 & 30.43 & 46.86 & 72.60 & 80.42 & 97.01 \\
\hline 127.10 & 141.69 & 174.82 & 179.85 & 194.62 & 196.71 \\
\hline 214.97 & 228.62 & 252.84 & 273.36 & 297.52 & 300.78 \\
\hline 332.86 & 367.05 & 422.06 & 445.87 & 468.40 & 494.89 \\
\hline 526.28 & 554.24 & 579.26 & 590.85 & 623.98 & 745.84 \\
\hline 823.64 & 849.28 & 885.35 & 948.35 & 967.38 & 987.14 \\
\hline 1011.28 & 1050.77 & 1085.15 & 1132.70 & 1148.54 & 1153.14 \\
\hline 1160.15 & 1172.36 & 1186.76 & 1230.18 & 1234.61 & 1254.30 \\
\hline
\end{tabular}




$\begin{array}{llllll}1307.94 & 1310.41 & 1315.08 & 1392.90 & 1407.31 & 1459.53 \\ 1461.81 & 1475.90 & 1486.71 & 1496.46 & 1510.04 & 1516.45 \\ 1519.74 & 1523.12 & 1528.91 & 3000.23 & 3001.43 & 3020.63 \\ 3026.66 & 3085.50 & 3089.96 & 3110.30 & 3126.67 & 3160.64 \\ 3164.50 & 3531.86 & 3561.16 & & & \end{array}$

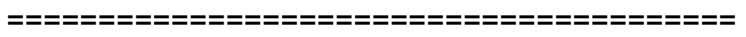
11

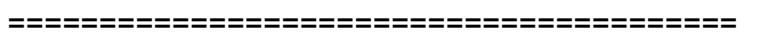

$\begin{array}{cccccc}148.08 & 371.14 & 611.44 & 753.19 & 760.96 & 818.54 \\ 881.90 & 893.71 & 930.94 & 974.52 & 1018.60 & 1054.12 \\ 1136.31 & 1199.67 & 1217.19 & 1238.00 & 1301.35 & 1311.12 \\ 1342.39 & 1490.74 & 1498.75 & 1516.63 & 1689.27 & 3018.24 \\ 3032.60 & 3060.75 & 3070.88 & 3084.40 & 3118.37 & 3212.08\end{array}$

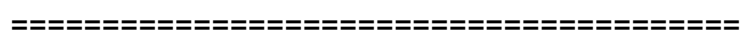
12

\begin{tabular}{|c|c|c|c|c|c|}
\hline 179.43 & 285.03 & 375.92 & 458.15 & 483.15 & 674.69 \\
\hline 755.24 & 821.57 & 835.67 & 887.15 & 905.94 & 936.36 \\
\hline 987.61 & 1026.47 & 1074.04 & 1082.49 & 1151.98 & 1171.21 \\
\hline 1253.62 & 1270.59 & 1290.59 & 1333.08 & 1354.76 & 1376.58 \\
\hline 1387.35 & 1483.49 & 1489.88 & 1499.73 & 1511.01 & 1739.59 \\
\hline 3007.39 & 3020.89 & 3031.87 & 3035.46 & 3055.26 & 3058.65 \\
\hline 3080.31 & 3088.16 & 3141.12 & & & \\
\hline
\end{tabular}

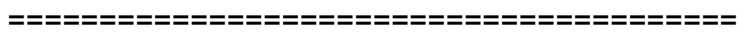

13

\begin{tabular}{|c|c|c|c|c|c|}
\hline 112.91 & 231.26 & 282.23 & 326.77 & 351.07 & 410.98 \\
\hline 558.75 & 647.29 & 734.59 & 764.35 & 815.56 & 847.92 \\
\hline 864.90 & 910.82 & 48.56 & 986.80 & 1006.10 & 1037.47 \\
\hline 1086.24 & 1125.12 & 1160.18 & 1211.75 & 1234.88 & 1261.50 \\
\hline 300. & 1312 & 342 & 1362 & 137 & 39 \\
\hline 1405 & 1481 & $148 \varepsilon$ & 149 & 15 & 1515.62 \\
\hline 1753 & 3007.08 & 3013.41 & 3021.87 & 3031.86 & 3038.36 \\
\hline 30 & 305 & 3058.13 & $307 t$ & .24 & 3120.28 \\
\hline
\end{tabular}

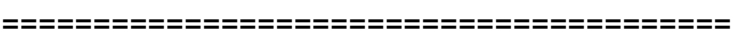

14

\begin{tabular}{|c|c|c|c|c|c|}
\hline 155.75 & 194.86 & 227.41 & 250.12 & 319.27 & 341.99 \\
\hline 431.22 & 495.69 & 525.91 & 572.42 & 728.60 & 767.06 \\
\hline 799.36 & 834.45 & 847.93 & 871.37 & 905.65 & 980.24 \\
\hline 994.00 & 1006.75 & 1034.71 & 1079.87 & 1108.40 & 1113.82 \\
\hline 1160.25 & 1205.25 & 1231.64 & 1269.51 & 1284.38 & 1302.61 \\
\hline
\end{tabular}

$\begin{array}{llllll}1315.80 & 1354.76 & 1360.88 & 1375.37 & 1386.68 & 1398.38 \\ 1403.78 & 1473.39 & 1495.86 & 1498.23 & 1502.60 & 1512.61 \\ 1518.65 & 1746.76 & 2973.72 & 3019.29 & 3021.84 & 3025.91 \\ 3033.70 & 3042.04 & 3042.54 & 3057.73 & 3066.26 & 3072.58 \\ 3078.68 & 3086.79 & 3128.13 & & & \end{array}$

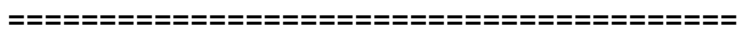

15

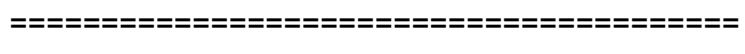

$\begin{array}{llllll}89.26 & 164.57 & 177.63 & 212.00 & 244.09 & 307.53\end{array}$

$\begin{array}{llllll}337.57 & 392.55 & 430.19 & 485.19 & 545.24 & 559.53\end{array}$

$\begin{array}{llllll}713.74 & 765.95 & 775.65 & 797.68 & 803.90 & 841.13\end{array}$

$\begin{array}{llllll}912.47 & 937.67 & 953.28 & 983.34 & 1031.26 & 1034.74\end{array}$

$\begin{array}{llllll}1062.47 & 1081.37 & 1100.97 & 1123.86 & 1177.63 & 1198.82\end{array}$

$\begin{array}{llllll}1217.12 & 1247.98 & 1281.19 & 1307.93 & 1317.16 & 1330.00\end{array}$

$\begin{array}{llllll}1350.70 & 1355.49 & 1364.94 & 1387.74 & 1396.26 & 1408.05\end{array}$

$\begin{array}{llllll}1417.62 & 1471.68 & 1494.05 & 1498.87 & 1501.10 & 1503.88\end{array}$

$\begin{array}{llllll}1511.77 & 1520.15 & 1756.60 & 2983.08 & 3010.01 & 3026.06\end{array}$

$\begin{array}{llllll}3027.23 & 3031.02 & 3035.25 & 3042.14 & 3043.93 & 3047.79\end{array}$

$\begin{array}{llllll}3072.49 & 3072.58 & 3074.31 & 3082.94 & 3086.98 & 3111.56\end{array}$

\begin{tabular}{|c|c|c|c|c|c|}
\hline \multicolumn{6}{|l|}{ J } \\
\hline$====$ & $:====$ & $====$ & $====$ & $===$ & $=$ =ニニニ= \\
\hline 27.62 & 42.63 & 48.79 & 57.35 & 63.75 & 73.50 \\
\hline 104.17 & 126.38 & 135.73 & 168.81 & 180.31 & 192.48 \\
\hline 225.14 & 227.89 & 239.89 & 249.76 & 266.57 & 277.76 \\
\hline 280.49 & 291.05 & 293.79 & 310.63 & 333.23 & 342.68 \\
\hline 356.58 & 370.27 & 375.63 & 417.63 & 424.35 & 437.14 \\
\hline 454.31 & 469.16 & 473.25 & 478.36 & 496.42 & 527.18 \\
\hline 618.98 & 628.85 & 661.20 & 698.63 & 726.69 & 742.37 \\
\hline 759.83 & 770.43 & 787.63 & 790.39 & 843.86 & 852.26 \\
\hline 864.58 & 899.70 & 926.08 & 934.51 & 938.79 & 939.75 \\
\hline 942.89 & 975.85 & 982.55 & 988.17 & 995.07 & 997.68 \\
\hline 1014.93 & 1047.05 & 1063.32 & 1063.65 & 1067.28 & 1071.31 \\
\hline 1079.67 & 1124.57 & 1176.70 & 1196.51 & 1213.46 & 1220.45 \\
\hline 1244.45 & 1266.84 & 1277.78 & 1282.68 & 1292.65 & 1299.57 \\
\hline 1329.75 & 1352.21 & 1369.12 & 1391.87 & 1411.28 & 1416.67 \\
\hline 1417.03 & 1421.95 & 1441.15 & 1445.95 & 1483.15 & 1484.57 \\
\hline 1501.92 & 1503.57 & 1504.85 & 1505.33 & 1506.26 & 1509.76 \\
\hline 1510.98 & 1520.52 & 1523.46 & 1532.80 & 1544.18 & 1546.57 \\
\hline 1640.87 & 1650.43 & 1729.27 & 1819.84 & 3051.54 & 3052.78 \\
\hline 3055.01 & 3059.32 & 3059.76 & 3066.37 & 3121.02 & 3122.87 \\
\hline 3124.25 & 3134.62 & 3135.48 & 3136.35 & 3143.63 & 3151.65 \\
\hline
\end{tabular}




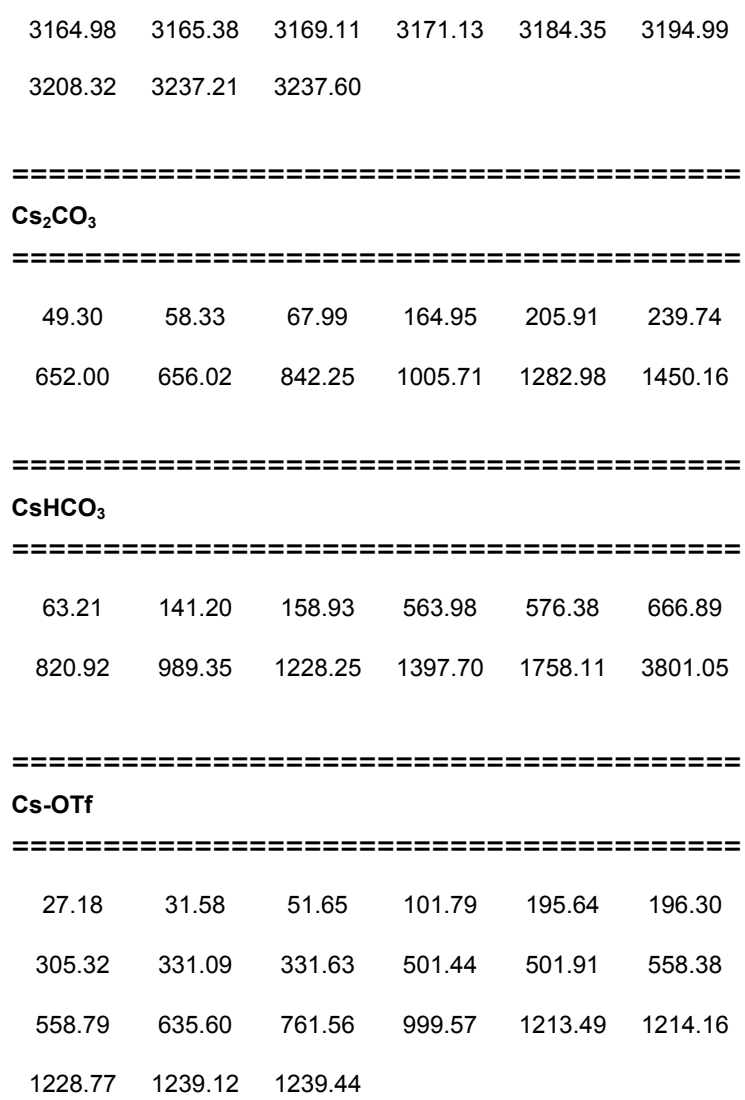

$\begin{array}{rrrrrr}60.59 & 191.74 & 192.72 & 289.33 & 331.17 & 331.42 \\ 497.77 & 498.24 & 555.06 & 555.22 & 621.34 & 737.54 \\ 997.42 & 1177.35 & 1177.95 & 1224.49 & 1254.64 & 1255.52\end{array}$

ニニニニニニニニニニニニニニニニニニニニニニニニニニニニニニニニニニニニニニニニ OTf .

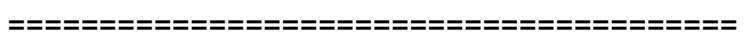

$\begin{array}{rrrrrr}45.40 & 93.71 & 137.75 & 181.63 & 183.06 & 290.38 \\ 342.73 & 343.09 & 543.22 & 543.54 & 588.41 & 766.81 \\ 928.99 & 974.94 & 1013.80 & 1208.02 & 1275.95 & 1276.90\end{array}$




\section{Supplemental References}

(1) Stanislawski, P. C.; Willis, A. C.; Banwell, M. G. New Protocols for the Assembly of the Tetracyclic Framework Associated with the Aromatic Erythrina Alkaloids. Org. Lett. 2006, 8, 2143-2146.

(2) Hioki, Y.; Okano, K.; Mori, A. Generation of Cycloalkynes through Deprotonation of Cyclic Enol Triflates with Magnesium Bisamides. Chem. Commun. 2017, 53, 2614-2617.

(3) Lim, B.-Y.; Jung, B.-E.; Cho, C.-G. Ene-Hydrazide from Enol Triflate for the Regioselective Fischer Indole Synthesis. Org. Lett. 2014, 16, 4492-4495.

(4) Wolter, M.; Klapars, A.; Buchwald, S. L. Synthesis of N-Aryl Hydrazides by Copper-Catalyzed Coupling of Hydrazides with Aryl lodides. Org. Lett. 2001, 3, 3803-3805.

(5) Ma, D.; Zhang, Y.; Yao, J.; Wu, S.; Tao, F. Accelerating Effect Induced by the Structure of a-Amino Acid in the Copper-Catalyzed Coupling Reaction of Aryl Halides with a-Amino Acids. Synthesis of BenzolactamV8. J. Am. Chem. Soc. 1998, 120, 12459-12467.

(6) Jiang, L.; Lu, X.; Zhang, H.; Jiang, Y.; Ma, D. Cul/4-Hydro-L-Proline as a More Effective Catalytic System for Coupling of Aryl Bromides with N-Boc Hydrazine and Aqueous Ammonia. J. Org. Chem. 2009, 74, 4542-4546.

(7) Fagan, P. J.; Hauptman, E.; Shapiro, R.; Casalnuovo, A. Using Intelligent/Random Library Screening To Design Focused Libraries for the Optimization of Homogeneous Catalysts: Ullmann Ether Formation. J. Am. Chem. Soc. 2000, 122, 5043-5051.

(8) Zhou, W.; Fan, M.; Yin, J.; Jiang, Y.; Ma, D. Cul/Oxalic Diamide Catalyzed Coupling Reaction of (Hetero)Aryl Chlorides and Amines. J. Am. Chem. Soc. 2015, 137, 11942-11945.

(9) Bhunia, S.; Pawar, G. G.; Kumar, S. V.; Jiang, Y.; Ma, D. Selected Copper-Based Reactions for C-N, CO, C-S, and C-C Bond Formation. Angew. Chem. Int. Ed. 2017, 56, 16136-16179.

(10) Bhuniya, D.; Singh, V. K. Homochiral Lithium Amides: Enantioselective Deprotonation of Cyclohexene Oxide. Synth. Commun. 1994, 24, 375-385.

(11) Qiu, Y.; Liu, Y.; Yang, K.; Hong, W.; Li, Z.; Wang, Z.; Yao, Z.; Jiang, S. New Ligands That Promote CrossCoupling Reactions between Aryl Halides and Unactivated Arenes. Org. Lett. 2011, 13, 3556-3559.

(12) Fan, M.; Zhou, W.; Jiang, Y.; Ma, D. Assembly of Primary (Hetero)Arylamines via Cul/Oxalic DiamideCatalyzed Coupling of Aryl Chlorides and Ammonia. Org. Lett. 2015, 17, 5934-5937.

(13) Roy, A. S.; Tuononen, H. M.; Rath, S. P.; Ghosh, P. First Ruthenium Complex of Glyoxalbis $(N-$ Phenyl)osazone $\left(\mathrm{LNHPh}_{2}\right)$ : Synthesis, X-ray Structure, Spectra, and Density Functional Theory Calculations of $\left(\mathrm{L}^{\mathrm{NHPh}} \mathrm{H}_{2}\right) \mathrm{Ru}\left(\mathrm{PPh}_{3}\right)_{2} \mathrm{Cl}_{2}$. Inorg. Chem. 2007, 46, 5942-5948.

(14) Parr, R. G.; Yang, W. Density-Functional Theory of Atoms and Molecules; Oxford University Press, 1994; pp 1-352.

(15) Bochevarov, A. D.; Harder, E.; Hughes, T. F.; Greenwood, J. R.; Braden, D. A.; Philipp, D. M.; Rinaldo, D.; Halls, M. D.; Zhang, J.; Friesner, R. A. Jaguar: A High-Performance Quantum Chemistry Software Program with Strengths in Life and Materials Sciences. Int. J. Quantum Chem. 2013, 113, 2110-2142.

(16) Slater, J. C.; Phillips, J. C. Quantum Theory of Molecules and Solids Vol. 4: The Self-Consistent Field for Molecules and Solids. Physics Today 1974, 27, 49-50.

(17) Vosko, S. H.; Wilk, L.; Nusair, M. Accurate Spin-Dependent Electron Liquid Correlation Energies for Local Spin Density Calculations: A Critical Analysis. Can. J. Phys. 1980, 58, 1200-1211.

(18) Becke, A. D. Density-Functional Exchange-Energy Approximation with Correct Asymptotic Behavior. Phys. Rev. A 1988, 38, 3098-3100.

(19) Becke, A. D. Density-Functional Thermochemistry. III. The Role of Exact Exchange. J. Chem. Phys. 1993, $98,5648-5652$. 
(20) Lee, C.; Yang, W.; Parr, R. G. Development of the Colle-Salvetti Correlation-Energy Formula into a Functional of the Electron Density. Phys. Rev. B 1988, 37, 785-789.

(21) Grimme, S.; Antony, J.; Ehrlich, S.; Krieg, H. A Consistent and Accurate Ab Initio Parametrization of Density Functional Dispersion Correction (DFT-D) for the 94 Elements H-Pu. J. Chem. Phys. 2010, 132, 154104.

(22) Hehre, W. J.; Ditchfield, R.; Pople, J. A. Self-Consistent Molecular Orbital Methods. XII. Further Extensions of Gaussian-Type Basis Sets for Use in Molecular Orbital Studies of Organic Molecules. J. Chem. Phys. 1972, 56, 2257-2261.

(23) Hay, P. J.; Wadt, W. R. Ab Initio Effective Core Potentials for Molecular Calculations. Potentials for the Transition Metal Atoms Sc to Hg. J. Chem. Phys. 1985, 82, 270-283.

(24) Wadt, W. R.; Hay, P. J. Ab Initio Effective Core Potentials for Molecular Calculations. Potentials for Main Group Elements Na to Bi. J. Chem. Phys. 1985, 82, 284-298.

(25) Hay, P. J.; Wadt, W. R. Ab Initio Effective Core Potentials for Molecular Calculations. Potentials for K to Au Including the Outermost Core Orbitals. J. Chem. Phys. 1985, 82, 299-310.

(26) Dunning, T. H., Jr. Gaussian Basis Sets for Use in Correlated Molecular Calculations. I. The Atoms Boron through Neon and Hydrogen. J. Chem. Phys. 1989, 90, 1007-1023.

(27) Woon, D. E.; Dunning, T. H., Jr. Gaussian Basis Sets for Use in Correlated Molecular Calculations. III. The Atoms Aluminum Through Argon. J. Chem. Phys. 1993, 98, 1358-1371.

(28) Marten, B.; Kim, K.; Cortis, C.; Friesner, R. A.; Murphy, R. B.; Ringnalda, M. N.; Sitkoff, D.; Honig, B. New Model for Calculation of Solvation Free Energies: Correction of Self-Consistent Reaction Field Continuum Dielectric Theory for Short-Range Hydrogen-Bonding Effects. J. Phys. Chem. 1996, 100, 11775-11788.

(29) Edinger, S. R.; Cortis, C.; Shenkin, P. S.; Friesner, R. A. Solvation Free Energies of Peptides: Comparison of Approximate Continuum Solvation Models with Accurate Solution of the Poisson-Boltzmann Equation. J. Phys. Chem. B 1997, 101, 1190-1197.

(30) Friedrichs, M.; Zhou, R.; Edinger, S. R.; Friesner, R. A. Poisson-Boltzmann Analytical Gradients for Molecular Modeling Calculations. J. Phys. Chem. B 1999, 103, 3057-3061.

(31) Jones, G. O.; Liu, P.; Houk, K. N.; Buchwald, S. L. Computational Explorations of Mechanisms and LigandDirected Selectivities of Copper-Catalyzed Ullmann-Type Reactions. J. Am. Chem. Soc. 2010, 132, 62056213.

(32) Creutz, S. E.; Lotito, K. J.; Fu, G. C.; Peters, J. C. Photoinduced Ullmann C-N Coupling: Demonstrating the Viability of a Radical Pathway. Science 2012, 338, 647-651.

(33) Ptasińska, S.; Gschliesser, D.; Bartl, P.; Janik, I.; Scheier, P.; Denifl, S. Dissociative Electron Attachment to Triflates. J. Chem. Phys. 2011, 135, 214309-214315. 